\title{
Search for CP Symmetry Violation in the
} 3-Pion Decay Mode of the K-Zero Meson

\author{
A THESIS \\ SUBMITTED TO THE FACULTY OF THE GRADUATE SCHOOL \\ OF THE UINIVERSITY OF MINNESOTA \\ BY \\ Nancy Lee Grossman \\ IN PARTIAL FULFILLMENT OF THE REQUIREMENTS \\ FOR THE DEGREE OF \\ DOCTOR OF PHILOSOPHY
}


thos

(C) Nancy Lee Grossman 1990

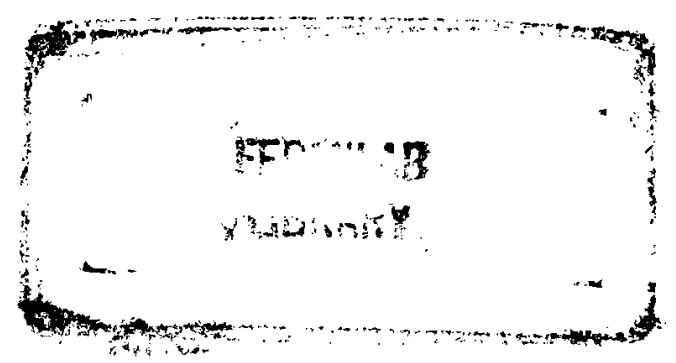




\title{
Search for CP Symmetry Violation in the 3-Pion Decay Mode of the K-Zero Meson
}

\author{
by Nancy Lee Grossman \\ Under the supervision of Professor Kenneth J. Heller
}

\begin{abstract}
Experimental measurements of $\mathrm{CP}$ symmetry violating parameters can make important contributions to our understanding of the electroweak interaction. At present the only well known values of CP symmetry violating parameters are determined from $\mathrm{K} \rightarrow 2 \pi$ decays. The theoretical parameter of interest is $\epsilon^{\prime}$ which measures $\mathrm{CP}$ symmetry violation in the decay matrix element of the neutral kaon. Most experiments measure $\eta_{+-} / \eta_{0 o}$ (or equivalently $\epsilon^{\prime} / \epsilon$ ). The advantage of measuring $\eta_{+-}$in $\mathrm{K} \rightarrow 3 \pi$ decay is that the theoretical predictions of $\epsilon_{+-}^{\prime}$ vary greatly. In the Kobayshi-Maskawa model $\eta_{+-0}$ is within a few percent of $\eta_{+-}$, but in other models it could be $50 \%$ or $100 \%$ larger. This is the first high statistics search for CP symmetry violation in neutral kaon decays to $\pi^{+} \pi^{-} \pi^{\mathbf{0}}$. It has the sensitivity to measure $\eta_{+-0}$ if it is unexpectedly large. The final sample was over 122,000 kaons decaying in this mode. No CP symmetry violation was observed, and thus a new limit was set on $\eta_{+-0}$. The final result was:

$$
\begin{aligned}
& \operatorname{Re}\left\{\eta_{+-0}\right\}=-0.229 \pm 0.046 \text { (stat) } \pm 0.023 \text { (syst) } \\
& \operatorname{Im}\left\{\eta_{+-0}\right\}=0.021 \pm 0.023 \text { (stat) } \pm 0.012 \text { (syst), }
\end{aligned}
$$

with $\mathcal{D}_{\mathrm{KK}}$, the dilution factor, as 0.5 . With the constraint $\operatorname{Re}\left\{\eta_{+-0}\right\} \approx \operatorname{Re}\left\{\eta_{+}\right\}=$ 0.0016 and the assumption that $\mathcal{D}_{\mathrm{KK}}=0.5$,

$$
\operatorname{Im}\left\{\eta_{+-0}\right\}=-0.021 \pm 0.025 \text { (stat) } \pm 0.012 \text { (syst). }
$$
\end{abstract}




\section{Acknowledgements}

High energy physics experiments are a collaborative effort. They depend on the talents, experience and cooporation of many people. This experiment would not have been possible without the many Fermilab personnel who operate the accelerator and beamlines. Special thanks goes to Roger Tokarek, our beamline physicist, who worked as our Fermilab liason physicist.

Gordon Thomson, as spokesman for the experiment, provided the much needed optimism and support necessary to embark on such a difficult experiment. My advisor, Ken Heller, was indispensible in getting the experiment set up and running. He was also a dependable source of new ideas, whether I wanted them or not.

Tom Devlin's knowledge of statistics and physics in general was of great help in the final analysis. He, Mike Shupe and Mike Longo helped us during data-taking with their experience in running experiments. Andy Beretvas ended up with the thankless position of "graduate student/professor liason physicist". His knowledge of physics and physicists was indispensable.

Prisca Cushman and Cat James kindly took several shifts for us on short notice when we were desperate for help. Similarly the efforts of Alex Pal and Tony Carracappa were appreciated in helping set up the experiment and take shifts. Tom Diehl, Jeff Duryea and Pak Ming Ho also helped us in these respects, and they remained at Fermilab for the following experiment E756. Because of this they had to endure my moods during the data analysis and they helped me deal with my lengthly graduate school term.

My fellow thesis student, Keith Thorne, helped by running all those horrid 4:30am shifts and by providing checks on my analysis through his related thesis analysis. I would also like to thank the two post-docs who assisted us in the experiment. Keith Krueger organized the electron-positron lead glass calibration

runs and analysis. Scott Teige helped set up the data summary tapes and converted our code from the Cyber to the VAX.

As for my fellow vampire, Pete Border, not enough can be said. He has been my sounding board from that first vampire shift together. He and Jeff Duryea helped me through the toughest times in my analysis. Their companionship has been invaluable. 
I can not forget the support given to me by those I love: Mark Schmitz, who actually married me during this ordeal, bless his heart; my mom away from home, Ruth Schmitz, who cooked me all those great meals; and, most of all, my parents, for whom this has probably been harder on than me...maybe. 


\section{Table of Contents}

Abstract
Acknowlgements
List of Diagrams
List of Tables
Chapter One: $C P$ Symmetry Violation Theory.................................................

1.1 Introduction 1

1.2 Kaon Phenomonolgy 3

1.2.1 CP Symmetry Violation in the $\pi \pi$ Channel 6

1.3 Theoretical Models 8

1.3.1 The Kobayashi-Moskawa Mode1 9

$\begin{array}{ll}\text { 1.3.2 The Superweak Model } & 13\end{array}$

$\begin{array}{ll}\text { 1.3.3 The Weinberg-Higgs Mode } & 14\end{array}$

1.3.4 The Left-Right Symmetric Model 15

1.4 Experimental Values 16

1.5 Experimental Approach 19

Chapter Two: Beamlines........................................................................... 23

2.1 Introduction 23

2.2 Enclosure PC1 24

2.3 Enclosure PC2 25

2.4 Enclosure PC3 26

Chapter Three: The Spectrometer........................................................ 30

$\begin{array}{ll}3.1 \text { Introduction } & 30\end{array}$

3.2 Multi Wire Proportional Chambers (MWPC's) 33

3.2.1 MWPC Efficiencies $\quad 34$

3.2.2 MWPC Double Hit Probablies $\quad 35$

3.3 The Lead Glass Array 36

3.3.1 Lead Glass Array Energy Resolution 37

3.4 A and B Hodoscopes 39

3.4.1 A and B Hodoscopes Efficiencies and Noise 39

3.5 Pi Hodoscopes 41 
3.5.1 Pi Hodoscope Noise 42

Chapter Four: Triggers and Trigger Processors................................................ 45

4.1 Introduction 45

4.2 Electronic Triggers 47

4.3 Memory Look Up Unit (MLU) Trigger Processor 51

4.3.1 Introduction 51

4.3.2 Design : First Level MLU Trigger Processor 52

4.3.3 Design : Second Level MLU Trigger Processor 52

4.3.4 MLU Trigger Processor Efficiencies 52

4.4 Charged Momemtum Ratio (CMR) Trigger Processor 54

4.4.1 Introduction 54

4.4.2 Design : First Level CMR trigger processor 54

4.4.3 Design : Second Level CMR Trigger Processor 58

4.4.4 CMR Trigger Processor Efficiencies 59

4.5 Glass Cluster Finding (GCF) Trigger Processor 62

4.5.1 Introduction 62

4.5.2 Design : Veto Boards 64

4.5.3 Design : Matrix Boards 68

4.5.4 Design : Summer Board 71

4.5.5 Glass Cluster Finding Trigger Proceesor Efficiencies 71

Chapter Five: Data Acquisition.................................................................... 74

$\begin{array}{ll}5.1 \text { General } & 74\end{array}$

5.2 Apparatus Allignment $\quad 75$

5.3 The Readout Procedure $\quad 75$

5.3.1 MWPC's $\quad 76$

5.3.2 ADC's and TDC's 77

$\begin{array}{ll}\text { 5.3.3 Latches and Scalers } & 77\end{array}$

5.3.4 Between Spill Readout $\quad 78$

5.4 Recording Event Data 78

5.5 Electron Positron Data 78

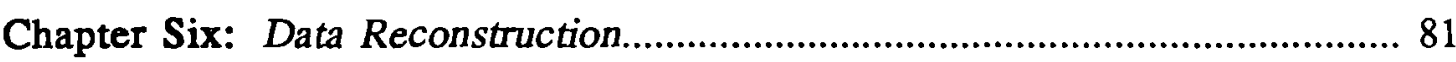

$\begin{array}{ll}6.1 \text { Introduction } & 81\end{array}$

6.2 Determining Apparatus Positions 81 
6.2.1 Chamber Positions 83

6.2.2 Beam and Target Positions 84

6.2.3 V1 and DK Counter Positions 88

6.2.4 Hodoscope Positions $\quad 89$

6.2.5 Analysis Magnets M2 and M3 89

6.2.6 Glass Positions 90

6.3 Magnetic Fields $\quad 91$

6.3.1 Magnet Fringe Fields 94

6.4 Determining Lead Glass Calibration Constants 95

6.5 The Reconstruction Program 98

$\begin{array}{ll}\text { 6.5.1 Introduction } & 98\end{array}$

6.5.2 Charged Track Reconstruction and Data Selection 98

6.5.3 Photon Shower Reconstruction 102

6.5.3.1 X,Y Position of Photon Showers 109

6.5.3.1.1 Non-Hole Photon Showers 110

6.5.3.1.1 Hole Photon Showers 110

Chapter Seven: The Monte Carlo............................................................ 113

7.1 Introduction 113

7.2 General Description $\quad 114$

$\begin{array}{ll}\text { 7.2.1 Data Bases } & 114\end{array}$

7.2.2 Neutral Particle Decay 115

7.2.3 The Trigger 116

7.3 Multiple Coulomb Scattering and Double Hits 116

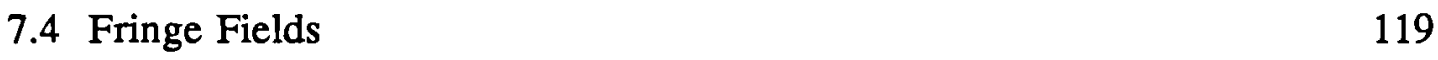

$\begin{array}{ll}7.5 \text { Efficiencies and Noise } & 119\end{array}$

$\begin{array}{ll}\text { 7.6 The Shower Monte Carlo } & 120\end{array}$

$\begin{array}{ll}\text { 7.6.1 Introduction } & 120\end{array}$

$\begin{array}{ll}\text { 7.6.2 Shower Shapes } & 121\end{array}$

7.6.3 Energy Losses $\quad 122$

7.6.4 TDC Thresholds $\quad 125$

Chapter Eight: Data Selection........................................................................ 126

8.1 Introduction 126

$\begin{array}{ll}8.2 \text { Signals and Background } & 126\end{array}$ 
$\begin{array}{ll}\text { 8.2.1 } \mathrm{K}_{3 \pi} \text { Sample } & 127\end{array}$

$\begin{array}{ll}\text { 8.2.2 } \mathrm{K}_{2 \pi} \text { Sample } & 127\end{array}$

8.3 Common $\mathrm{K}_{2 \pi} / \mathrm{K}_{3 \pi}$ Data Requirements 128

8.4 Further $\mathrm{K}_{3 \pi}$ Data Requirements 139

8.4.1 $\mathrm{K}_{3 \pi}$ Photon Selection Criteria 140

8.4.2 $\mathrm{K}_{3 \pi}$ Final Data Selection 146

8.4.3 $\mathrm{K}_{3 \pi}$ Dalitz Plot 149

Chapter Nine: Physics Analysis.................................................................... 155

9.1 General 155

$9.2 \chi^{2}$ Minimization 158

9.3 Maximum Likelihood 158

$9.4 \mathrm{~K} \rightarrow 2 \pi$ Measurements $\quad 159$

9.4.1 $\mathrm{K}_{\mathrm{s}}$ Lifetime 160

9.4.2 Normalization Values of Kaons on Target 162

9.5 The $\eta_{+-0}$ Measurement 164

9.5.1 Monte Carlo Studies of the Measurement Method 165

$\begin{array}{ll}9.5 .2 \text { Biases } & 167\end{array}$

9.5.3 Maximum Likelihood and $\eta_{+-0} \quad 170$

9.5.4 $\chi^{2}$ Acceptance Method 173

9.5.5 Dual Beam Results 175

9.5.6 Systematic Uncertainties 178

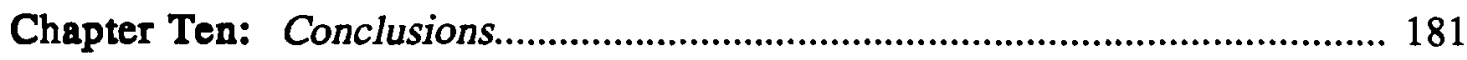

10.1 The Result 181

10.2 Implications of the Results 183

10.3 The Experimental Technique/Future Experiments 184

Appendix A: Complete Enclosure and Collimator Diagrams......................... 188

Appendix B: Radiation Lengths of Material in Experimental Apparatus...... 192

Appendix C: Collimator Produced Kaon Background..................................... 194

Appendix D: Dilution Factor................................................................... 197

Appendix E: CP-Conserving Decay............................................................ 199

Appendix F: Tabulated Data and Acceptance........................................... 200

F.1 Monte Carlo-Data Dalitz Distributions 201

F.2 Tabulated Data 204

vii 
F.3 Tabulated Acceptance 209

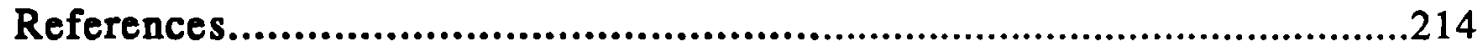

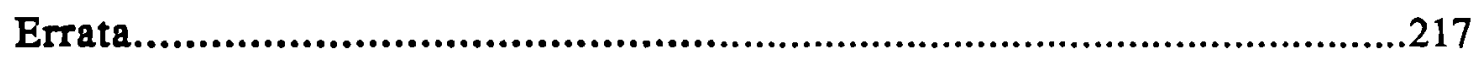




\section{Diagrams}

Chapter One: CP Symmetry Violation Theory

Figure 1.1 Mass Matrix Feynman Diagrams

Figure 1.2 Decay Matrix Feynman Diagrams

Figure 1.3 $\eta_{+-0}$ Experimental Values from 1972 to the Present

Figure 1.4 Experimental Values of $\operatorname{Im}\left\{\eta_{+-0}\right\}$ for $\operatorname{Re}\left\{\eta_{+-0}\right\}=0.0$

Figure 1.5 Expected Proper Time Ratio of Events for $\eta_{+-0}=\eta_{+-}$

Chapter Two: Beamlines

Figure 2.1 Enclosure PC1

Figure 2.2 Enclosure PC2

Figure 2.3 Enclosure PC3

Figure 2.4 E621 Collimator

Chapter Three: The Spectrometer

Figure 3.1 The Spectrometer: Plan View (not drawn to scale)

Figure 3.2 The Lead Glass Array

Figure 3.3 Lead Glass Resolution E/P Plots

Figure $3.4 \mathrm{~A}$ and $\mathrm{B}$ Hodoscope Arrays

Figure 3.5 Pi Hodosccopes Looking Downstream

Figure 3.6 Photon Non-veto Likelihoods

Chapter Four: Triggers and Trigger Processors

Figure 4.1 Charge Track Mometum Ratios for Lambdas and Kaons 46

Figure 4.2 Tracks of a Typical Particle Decay 49

Figure 4.3 E621 Trigger Schematic 48

Figure 4.4 MLU Layout $\quad 51$

Figure 4.5 B Hodoscopes 53

Figure 4.6 CMR Trigger Processor Layout $\quad 55$

Figure 4.7 Priority Encoder $\quad 57$

Figure 4.8 Arithmetic Logic Unit (ALU) 60

Figure 4.9 X Track Positions at C5 61

Figure 4.10 GCF Trigger Processor Boards and Their Signals 63

Figure 4.11 GCF Trigger Processor Lead Glass Numbering 64 
Figure 4.12 Veto Board 1: Pi Hodoscope Overlap Signal Information 66

Figure 4.13 Pi Hodoscope Overlap Vetoes 65

Figure 4.14 Unconditional Lead Glass Block Veto 65

Figure 4.15 GCF Trigger Processor Lead Glass Block Algorithm 69

Figure 4.16 Matrix Board: Finding Cluster Edges 70

Figure 4.17 The Summer Board 72

Chapter Six: Data Reconstruction

Figure 6.1 $\mathrm{X}$ and $\mathrm{Y}$ of the Charged Tracks at the Analysis Magnets 82

Figure 6.2 $\mathrm{X}$ and $\mathrm{Y}$ of the Photons at the Lead Glass 82

Figure 6.3 Chamber C5Y and C5X Residual 84

Figure 6.4 $\Theta_{x}$ and $\Theta_{y}$ of $K_{3 \pi}$ for Downstream East Data 85

Figure $6.5 \mathrm{~K}_{3 \pi}$ Projected to the Upstream Target for Downstream East Data 86

Figure $6.6 \mathrm{~K}_{3 \pi}$ Projected to Lead Glass Array for Downstream East Data 87

Figure 6.7 Z Vertex Distribution from all Triggers 88

Figure 6.8 Full $\mathrm{K}_{3 \pi} \mathrm{Z}$ Vertex Distribution $\quad 89$

Figure 6.9 The Effiective Bend Plane 91

Figure 6.10 The Effective Bend Plane for Two Magnets 92

Figure $6.11 \mathrm{~K}_{2 \pi}$ Mass 93

Figure $6.12 \pi^{+} \pi^{-}$Mass $\quad 93$

Figure 6.13 Fringe FIeld Comparisons for $\mathrm{K} \rightarrow 2 \pi$ Decays 94

Figure 6.14 Variations in Lead Glass Calibration Constants versus Time 97

$\begin{array}{lll}\text { Figure } 6.15 & \text { Pi-zero Mass } & 98\end{array}$

Figure $6.16 \pi \pi$ Invariant Mass $\quad 100$

Figure $6.17 \pi \mathrm{p}$ Invariant Mass 100

Figure 6.18 Collimator Cut Defining East and West for $\mathrm{K} \rightarrow 2 \pi$ Data 101

Figure 6.19 Higher Energy Photon Energy 103

Figure 6.20 Lower Energy Photon Energy 103

Figure 6.21 Photon Radii 104

Figure 6.22 Distance From the Pion track to the Nearest Shower 105

Figure 6.23 Smallest Distance From a Pion track to Chosen Photon Shower 106

Figure 6.24 Energy of the Highest Energy Extra Neutral Shower 107

Figure 6.25 Collimator Cut Defining East and West for $\mathrm{K}_{3 \pi}$ Data 108 
Figure 6.26 Uncorrected and Corrected Non-hole Positions vs Track Positions

Figure 6.27 Hole Regions

Figure 6.28 Region 1 Corrected Positions vs. Track Positions

112

Figure 6.29 Region 2 Corrected Positions vs. Track Positions

Chapter Seven: The Monte Carlo

Figure 7.1 Multiple Scattering Parameters

117

Figure $7.2 \sigma^{2}$ vs. $1 / \mathrm{p}^{2}$

118

Figure 7.3 $\sigma^{2}$ Data vs. $\sigma^{2}$ Monte Carlo

118

Figure 7.4 Radius vs. Energy for Data and Monte Carlo (corrected)

121

Figure 7.5 P vs. E Non-holes

123

Figure $7.6 \mathrm{P}$ vs. E Holes

Figure $7.7 \mathrm{E} / \mathrm{P}$ for $\mathrm{e}^{+} \mathrm{e}^{-}$(actual and corrected)

123

Figure 7.8 Hole Inner Regions

124

Chapter Eight: Data Selection

Figure 8.1 Slope Ratio Requirement

129

Figure $8.2 \chi^{2} / \mathrm{DOF}$

130

Figure 8.3 $\mathrm{P}_{\mathrm{T}}^{2}$

131

Figure 8.4 $\Theta$ of $K_{2 \pi}$ Decay for Upstream West Data

132

Figure 8.5 $\mathrm{K}_{2 \pi}$ Projected to the Upstream Target for Upstream West Data 133

Figure 8.6 $\mathrm{K}_{2 \pi}$ Projected to the Lead Glass Array for Upstream West Data 133

Figure $8.7 \Theta$ of $\mathrm{K}_{3 \pi}$ Decay for Upstream West Data

134

Figure 8.8 $\mathrm{K}_{3 \pi}$ Projected to the Upstream Target for Upstream West Data 134

Figure $8.9 \mathrm{~K}_{3 \pi}$ Projected to the Lead Glass Array for Upstream West Data 135

Figure $8.10 \mathrm{M}_{\pi^{+} \pi^{-}}$for $\mathrm{K} \rightarrow 2 \pi$ events

135

Figure 8.11 $\mathrm{M}_{\pi^{+} \pi^{-}}$for $\mathrm{K} \rightarrow 3 \pi$ events

136

Figure 8.12 Z Vertex for $\mathrm{K} \rightarrow 2 \pi$ events 137

Figure 8.13 Proper Time for $K \rightarrow 2 \pi$ events 137

Figure 8.14 Momentum of $\pi^{+}$for $\mathrm{K} \rightarrow 2 \pi$ events 138

Figure 8.15 Momentum of $\pi$ for $\mathrm{K} \rightarrow 2 \pi$ Decay 138

Figure 8.16 Charged Pion Opening Angle in $\mathrm{K} \rightarrow 2 \pi$ Decay 139

Figure $8.17 \mathrm{~K}_{2 \pi}$ Momentum Spectrum 
Figure 8.18 $\mathrm{K}_{3 \pi}$ Momentum Spectrum $\quad 140$

Figure 8.19 Photon Low Energy Cuts: Higher Energy Photon 141

Figure 8.20 Photon Low Energy Cuts: Lower Energy Photon 141

Figure 8.21 Mass of the $\pi^{\circ}$ (non-hole and hole events) 143

Figure 8.22 Photon Energies (non-hole and hole events) 143

Figure 8.23 Number of Blocks in Showers (non-hole and hole events) 144

Figure 8.24 Photon-Photon Opening Angle (non-hole and hole events) 145

Figure 8.25 Momentum of the $\pi^{0}$ (non-hole and hole events) 145

Figure 8.26 $\mathrm{M}_{\mathrm{e}+\mathrm{e}}$ in $\mathrm{K}->3 \pi$ Decay (no $\mathrm{M}_{\mathrm{e}+\mathrm{e}^{-}}$requirement) 146

Figure 8.27 Mass of $\mathrm{K}_{3 \pi} \quad 148$

Figure 8.28 Mass of $\pi^{\circ} \quad 149$

Figure 8.29 Z-Vertex in $\mathrm{K} \rightarrow 3 \pi$ Decay 151

Figure 8.30 Proper Time in $\mathrm{K} \rightarrow 3 \pi$ Decay 152

Figure 8.31 Lambda Mass in $\mathrm{K} \rightarrow 3 \pi$ Decay 152

Figure 8.32 $\mathrm{M}_{\mathrm{e}^{+} \mathrm{e}^{-}}$in $\mathrm{K} \rightarrow 3 \pi$ Decay (all cuts applied) 153

Figure 8.33 Charged Pion Opening Angle in $\mathrm{K} \rightarrow 3 \pi$ Decay 153

Figure 8.34 Momentum of $\pi^{+}$in $\mathrm{K} \rightarrow 3 \pi$ Decay 154

Figure 8.35 Momentum of $\pi$ in $\mathrm{K} \rightarrow 3 \pi$ Decay 154

Chapter Nine: Physics Analysis

Figure $9.1 \mathrm{~K}_{\mathrm{s}}$ Lifetime vs. Momentum 162

Figure $9.2 \mathrm{~K}_{3 \pi}$ Z-Vertex 0 to 20 Meters 165

Figure $9.3 \mathrm{~K}_{3 \pi}$ Z-Vertex: Monte Carlo Data Difference Plot 169

Chapter Ten: Results and Conclusions

Figure 10.1 Proper Time Ratio of Data: Both East and West Beams

Figure 10.2 Graph of Experimental Values of $\eta_{+-0}$ from 1972 to Present with this Experiment's results

Figure 10.3 Graph of Experimental Values of $\operatorname{Im}\left\{\eta_{+-0}\right\}$ with this Experiment's results for $\operatorname{Re}\left[\eta_{+-0}\right.$ Constrained to Zero

Appendix A: Complete Enclosure and Collimator Diagrams

Figure C.1 Enclosure PC1 (with dimensions) 188

Figure C.2 Enclosure PC2 (with dimensions) 189

Figure C.3 Enclosure PC3 (with dimensions) 190

Figure C.4 The Collimator (with dimensions) 191 
Appendix C: Collimator Produced Kaon Background

Figure C.1 Neutral Beam Angle in X for Upstream West Kaon Backgrounds 194

Figure C.2 Simulation of Collimator Kaon Background in Upstream West $\mathrm{K} \rightarrow 2 \pi$ Decay

Appendix D: Dilution Factor

Figure D.1 $\mathrm{K}^{+} / \mathrm{K}^{-}$Production Ratios versus $\mathrm{X}_{\mathrm{F}}$

198 


\section{Tables}

Chapter One: CP Symmetry Violation Theory

Table $1.1 \eta_{+-o}$ Experimental Values

Chapter Two: Beamlines

Table 2.1 Target Positions

Chapter Three: The Spectrometer

Table 3.1 Chamber Aperatures and Voltages

Table 3.2 Chamber Efficiencies

Table 3.3 Double Hit Probability

Table 3.4 A and B hodoscope Inefficiencies Determined from F. C5 Triggers

Chapter Four: Triggers and Trigger Processors

Table 4.1 Triggers and Prescaler Values 50

Table 4.2 CMR Trigger Comparitor Output Conditions 59

Chapter Five: Data Acquisition

Table 5.1 Eight Different Run Types

Chapter Six: Data Reconstruction

Table 6.1 Magnet Parameters for E621 93

Table 6.2 Extra Requirements Used on Calibration Constant Data Sample 96

Table 6.3 First Order Reconstruction of $\mathrm{K}_{3 \pi}$ and $\mathrm{K}_{2 \pi}$ Triggers 101

Table 6.4 General Photon Statistics

102

Chapter Seven: The Monte Carlo

Table 7.1 Values Varied Tape by Tape

Table 7.2 Values Kept Constant over the Data Sample 115

Table 7.3 Monte Carlo Noises and Efficiencies

Chapter Eight: Data Selection

Table 8.1 Preliminary $K_{3 \pi}$ and $K_{3 \pi}$ Data Selection

Table 8.2 Neutral Beam Phase Space Requirements

Table 8.3 Photon Shower Selection Criteria

Table $8.4 \mathrm{~K} \rightarrow 3 \pi$ Data Losses

Chapter Nine: Physics Analysis

Table 9.1 Ks Lifetime for Downstream East and Downstream West Targets 161 xiii 
Table 9.2 Total Number of $\mathrm{K} \rightarrow 2 \pi$ Decays

Table 9.3 Systematic Uncertainites in the Number of Kaons

Table 9.4 Single Beam Maximum Likelihood Results of $\eta_{+-0}$

Table 9.5 Single Beam $\chi^{2}$ Minimization Results of $\eta_{+-0}$

Table 9.6 Number of Events in Final $\mathrm{K} \rightarrow 3 \pi$ Sample

Table 9.7 Final Result of the Measurement of $\eta_{+-0} \quad 176$

Table 9.8 Correlation Matrices in the Measurement of $\eta_{+-0}$

Table 9.9 Systematic Uncertainties of $\eta_{+-0} \quad 179$

Chapter Ten: Conclusions

Table 10.1 Possible Design Improvements

Appendix B: Radiation Lengths of Material in Experimental Apparatus

Table B.1 Radiation Lengths of Material

Appendix C: Collimator Produced Kaon Background

Table C.1 Percentages of Collimator Produced Kaon Background

196

Appendix F: Tabulated Data and Acceptance

Table F.1 Monte Carlo-Data Dalitz Distribution (downstream west)

201

Table F.2 Monte Carlo-Data Dalitz Distribution (upstream west)

Table F.3 Monte Carlo-Monte Carlo Signal Dalitz Distribution (downstream)

Table F.4 Downstream West Data

Table F.5 Downstream East Data

205

Table F.6 Upstream West Data

206

Table F.7 Upstream East Data

207

Table F.8 Downstream West Acceptance 208

Table F.9 Downstream East Acceptance 210

Table F.10 Upstream West Acceptance 211

Table F.11 Upstream East Acceptance 


\section{Chapter One}

\section{CP Symmetry Violation Theory}

\subsection{Introduction}

Symmetries and conservation laws have long played an important role in physics. The simplest examples are the conservation of energy and momentum, which are due to the invariance of physical laws under translation in time and space respectively. These are macroscopic symmetries. In the domain of quantum phenomena there are also conservation laws corresponding to discrete transformations which leave a system invariant.

One of these is reflection in space or the "parity operation", P. The invariance of nature under $\mathbf{P}$ means that the mirror image of an experiment yields the same result in its reflected frame of reference as the original experiment in the original frame of reference. Under parity, left-handed becomes right-handed and thus left and right cannot be defined in an absolute sense if parity is conserved. The parity operation reverses momentum but not angular momentum. A second invariance in quantum mechanics called "charge conjugation", C, transforms particles into antiparticles. $C$ invariance requires that an experiment performed in an anti-world on anti-particles have the same outcome as one in our world performed on particles. A third transformation, $T$, reverses time. Under this transformation the direction of momenta and angular momenta are reversed. The three operations $C, P$ and $T$ can then be combined to form an invariant called CPT. According to the CPT theorem of Lüders and Pauli(1) a local field theory which is Lorentz invariant is also invariant under the combined operation CPT.

For a long time it was assumed that all elementary processes were invariant under the application of each of the three operations $\mathrm{C}, \mathrm{P}$ and $\mathrm{T}$ seperately. Lee and Yang ${ }^{(2)}$ questioned this assumption in 1956. They postulated that if charge conjugation were conserved, the long-lived $\mathrm{K}_{\ell}$ or $\mathrm{K}_{2}$ would be an eigenstate of $\mathrm{C}$ and consequently its decay into charge conjugate channels such as $\pi^{+} e^{-} v$ and $\pi^{-} e^{+} \bar{v}$ 
must be equally probable. An asymmetry in the rate of decay of the $\mathrm{K}_{\ell}$ into these decay modes would indicate a violation of $\mathrm{C}$ invariance.

Subsequent experiments demonstrated the violation of $\mathrm{P}$ and $\mathrm{C}$ invariance in weak decays of nuclei ${ }^{(3)}$ and of pions and muons ${ }^{(4)}$. The violation was seen in the longitudinal polarization of neutrinos emerging from a weak decay. Neutrinos are left-handed when they are particles and right-handed when they are antiparticles. Thus the application of $\mathrm{P}$ or $\mathrm{C}$ to a neutrino leads to an unphysical state. The combined operation CP, though, still appeared to be invariant in weak interactons ${ }^{(5)}$ since CP transforms a left-handed neutrino into a right-handed anti-neutrino and vice versa.

Assuming $C P$ invariance in weak interactions, Gell-Mann and Pais ${ }^{(6)}$ predicted a long-lived partner, $\mathrm{K}^{\mathrm{O}}{ }_{2}$, to the known $\mathrm{K}^{\mathrm{o}}{ }_{1}$ particle which had a short lifetime $\sim 10^{-10} \mathrm{sec}$. The $\mathrm{K}^{\mathrm{O}}{ }_{1}$ and $\mathrm{K}^{\mathrm{o}}{ }_{2}$ are mixtures of the two eigenstates of strangeness, $\mathrm{K}^{\mathrm{O}}(\mathrm{s}=+1)$ and $\overline{\mathrm{K}}^{\mathrm{O}}(\mathrm{s}=-1)$, produced in strong interactions. Weak interactions do not conserve strangeness, but if weak interactions were $\mathbf{C P}$ invariant, the physical particles when they decay should be eigenstates of $\mathrm{CP}$ since they decay weakly. These eigenstates are (with $\mathrm{CP}\left|\overline{\mathrm{K}}^{0}\right\rangle=\left|\mathrm{K}^{\circ}\right\rangle$ ):

$$
\begin{aligned}
& \mathrm{CP}\left|\mathrm{K}_{1}\right\rangle=\mathrm{CP}\left(\left|\mathrm{K}^{\circ}\right\rangle+\left|\overline{\mathrm{K}^{\circ}}\right\rangle\right) / \sqrt{2}=\left(\left|\mathrm{K}^{\circ}\right\rangle+\left|\overline{\mathrm{K}^{\circ}}\right\rangle\right) / \sqrt{2}=\left|\mathrm{K}_{1}\right\rangle \\
& \mathrm{CP}\left|\mathrm{K}_{2}\right\rangle=\mathrm{CP}\left(\left|\mathrm{K}^{\circ}\right\rangle-\left|\overline{\mathrm{K}^{\circ}}\right\rangle\right) / \sqrt{2}=\left(\left|\overline{\mathrm{K}^{\circ}}\right\rangle-\left|\mathrm{K}^{\circ}\right\rangle\right) / \sqrt{2}=-\left|\mathrm{K}_{2}\right\rangle
\end{aligned}
$$

For pions in a state with angular momentum zero, CP $\left|\pi^{+} \pi^{-}\right\rangle=\left|\pi^{+} \pi^{-}\right\rangle$, and thus the decay into $\pi^{+} \pi^{-}$is allowed for the $\mathrm{K}^{0}{ }_{1}$ but forbidden for the $\mathrm{K}^{\mathrm{o}}{ }_{2}$ because it violates CP. Gell-Mann and Pais postulated that $\mathrm{K}_{2}{ }_{2} \rightarrow \pi^{+} \pi^{-} \gamma$ or $3 \pi$ etc. Because three body phase space depresses the decay rate relative to two body phase space, this led them to predict a longer lifetime for the $\mathrm{K}_{2}^{0}$. Their theory also predicted a small mass difference betweeen $\mathrm{K}^{\circ}{ }_{1}$ and $\mathrm{K}_{2}{ }_{2}$. The long-lived $\mathrm{K}_{2}{ }_{2}$ was discovered $(7)$ shortly thereafter. No decays of the long-lived $\mathrm{K}_{2}^{\mathrm{O}}$ to two pions were seen, only decays to three or four particles, confirming Gell-Mann and Pais' predictions.

Then in 1964, Christenson, Cronin, Fitch and Turlay ${ }^{(8)}$ (CCFT) discovered that the long-lived neutral $\mathrm{K}$ meson, previously designated $\mathrm{K}_{2}^{\mathrm{o}}$, also decays to $\pi^{+} \pi^{-}$ 
violating $\mathrm{CP}$ conservation. This long-lived state was then called $\mathrm{K}_{\ell}$ since it no longer was identical to the $\mathrm{CP}$ eigenstate $\mathrm{K}_{2}{ }_{2}$. Similarly the short-lived state was called $\mathrm{K}_{\mathrm{s}}$. The branching ratio for the CP non-conserving decay $\mathrm{K}_{\ell} \rightarrow \pi^{ \pm} \pi^{\mp}$ was $\sim 2 \times 10^{-3}$ as measured by CCFT. Subsequent discoveries of the decay $K_{\ell} \rightarrow \pi^{\circ} \pi^{\circ}$ (9) and of a charge asymmetry in the decays $K_{\ell} \rightarrow \pi^{ \pm} e^{\mp} v$ and $K_{\ell} \rightarrow \pi^{ \pm} \mu^{\mp} v$ (10) confirmed CCFT results of $\mathrm{CP}$ non-invariance in weak interactions.

\subsection{Kaon Phenomonolgy}

The $25^{\text {th }}$ anniversary of the discovery of CP symmetry violation was last year. Much work experimentally and theoretically has been done in that time. Nowadays several models can account for the observed $\mathrm{CP}$ asymmetries. However all observations of $\mathrm{CP}$ non-invariance remain in the $\mathrm{K}$-meson system alone. Thus $\mathrm{I}$ shall start with a discussion of the $\mathrm{K}$ system formalism and then discuss the various models and their predictions for $\mathrm{K} \rightarrow 2 \pi$ and $\mathrm{K} \rightarrow 3 \pi \mathrm{CP}$ non-invariance.

In 1986 R. Turlay ${ }^{(11)}$ presented a status report on CP violation in the neutral $\mathrm{K}$ system. Much of what follows is derived from his concise report.

The wavefunction for a neutral kaon can be written as:

$$
\psi_{(\mathrm{t})}=\mathrm{a}(\mathrm{t})\left|\mathrm{K}^{\circ}\right\rangle+\mathrm{b}(\mathrm{t})\left|\overline{\mathrm{K}}^{\circ}\right\rangle
$$

where $a(t)$ and $b(t)$ are the time dependent amplitudes for the $\left|K^{\circ}\right\rangle$ and $\left|\bar{K}^{\circ}\right\rangle$ respectively. The time evolution of the state is given by the Schrodinger equation:

$$
i \mathrm{~d} \Psi / \mathrm{dt}=\mathscr{H} \Psi,
$$

where $\mathscr{H}$ is the Hamiltonian describing the decay processes. $\mathscr{H}$ is not hermitian but can be broken down into two $2 \times 2$ hermitian matrices. One writes:

$$
\mathscr{H}=\mathrm{M}-\frac{i \Gamma}{2}
$$

where $M$ is the mass matrix and $\Gamma$ the decay matrix. These two matrices can be expressed as:

$$
\left|\begin{array}{ll}
M_{11} & M_{12} \\
M_{12}^{*} & M_{22}
\end{array}\right| \text { and }\left|\begin{array}{ll}
\Gamma_{11} & \Gamma_{12} \\
\Gamma_{12}^{*} & \Gamma_{22}
\end{array}\right| \text {. }
$$

$M_{11}, M_{22}, \Gamma_{11}$, and $\Gamma_{22}$ must also be real due to hermicity.

The general symmetry principles corresponding to $\mathrm{C}, \mathrm{P}$ and $\mathrm{T}$ and the 
combinations $\mathrm{CP}$ and CPT further constrain the $\mathrm{M}$ and $\Gamma$ matrix elements.

$$
\begin{array}{lll}
\text { CPT Invariance } & & M_{11}=M_{22} \\
& & \Gamma_{11}=\Gamma_{22} \\
& & M_{12}, M_{21} \text { real } \\
\text { T Invariance } & \rightarrow-> & \Gamma_{12}, \Gamma_{21} \text { real } \\
& & M_{11}=M_{22}, \Gamma_{11}=\Gamma_{22} \rightarrow \text { CPT Invariance } \\
\text { CP Invariance } & -\rightarrow & M_{12}, M_{21}, \Gamma_{12}, \Gamma_{21} \text { real } \rightarrow \mathrm{T} \text { Invariance }
\end{array}
$$

Thus CP symmetry violation can be due to CPT non-invariance, $T$ noninvariance or both.

. Since the weak interaction was thought to conserve $\mathrm{CP}, \mathrm{CP}$ eigenstates $\mathrm{K}^{\circ}{ }_{1}$ and $\mathrm{K}_{2}^{0}$ were postulated as shown in equation 1.1 and 1.2. When in $1964 \mathrm{CCFT}$ observed $\mathrm{K}_{2}{ }_{2} \rightarrow \pi^{+} \pi^{-}$which violates $\mathrm{CP}$ conservation, the eigenstates of the weak Hamiltonian $\mathscr{H}$ were then redefined as the physical states $\mathrm{K}_{\ell}$ and $\mathrm{K}_{\mathrm{s}}$ with definite mass $(m)$ and decay width $(\gamma)$. The non-CP invariant Hamiltonian can be written as the diagonalized matrix $\mathrm{M}-\frac{i \Gamma}{2}$. Then:

$$
\mathrm{M}-\frac{i \Gamma}{2} \Psi=i \mathrm{~d} \Psi / \mathrm{dt}=\lambda \psi
$$

with:

$$
\psi(\mathrm{t})=\mathrm{e}^{-i \lambda \mathrm{t}} \psi
$$

and:

$$
\lambda_{\mathrm{s}}=m_{\mathrm{s}}-i \gamma_{\mathrm{s}} / 2, \lambda_{l}=m_{l}-i \gamma_{l} / 2
$$

The Eigenvalues are:

$$
\begin{aligned}
\left|K_{\mathcal{S}}^{o}\right\rangle & =\frac{1}{2 \sqrt{1+|\epsilon+\delta|^{2}}}\left[(1+\epsilon+\delta)\left|K^{o}\right\rangle+(1-\epsilon-\delta)\left|\bar{K}^{o}\right\rangle\right] \\
\left|K_{\ell}^{o}\right\rangle & =\frac{1}{2 \sqrt{1+|\epsilon-\delta|^{2}}}\left[(1+\epsilon-\delta)\left|K^{o}\right\rangle-(1-\epsilon+\delta)\left|\bar{K}^{o}\right\rangle\right]
\end{aligned}
$$

with: 


$$
\epsilon=\frac{-\operatorname{Im}\left(M_{12}\right)+i \operatorname{Im}\left(\Gamma_{12}\right)}{\left[i\left(m_{s}-m_{\ell}\right)+\left(\gamma_{s}-\gamma_{\ell}\right) / 2\right]}
$$

the $\mathrm{CP}$ symmetry violating parameter associated with $\mathrm{T}$ non-invariance and;

$$
\delta=\frac{1}{2} \frac{\left(\Gamma_{11}-\Gamma_{22}\right)+i\left(M_{11}-M_{22}\right)}{\left[i\left(m_{s}-m_{\ell}\right)+\left(\gamma_{s}+\gamma_{\ell}\right) / 2\right]}
$$

the CP symmetry violating parameter associated with CPT non-invariance. $\mathrm{m}_{\mathrm{s}}, \mathrm{m}_{\ell}$ and $\gamma_{s}, \gamma_{\ell}$ are the masses and the decay widths of the $K_{s}$ and $K_{\ell}$.

The non-orthogonality of the eigenvectors $K_{s}$ and $K_{\ell}$ is a measurement of $C P$ symmetry violation. The non-diagonal elements of the decay and mass matrix can be expressed by the first terms of a perturbative expansion:

$$
\Gamma_{12}=2 \pi \sum_{\xi} \rho_{F}\left\langle\overline{\mathrm{K}^{\mathrm{o}}}\left|\mathscr{H}_{\mathrm{wk}}\right| \mathscr{F}\right\rangle\left\langle\bar{F}\left|\mathscr{H}_{\mathrm{wk}}\right| \mathrm{K}^{\mathrm{o}}\right\rangle
$$

where the sum runs over all possible intermediate states $\mathscr{F}$ with phase space density $\rho_{s}$ and $\mathscr{H}_{w k}$ is the non-CP invariant weak Hamiltonian. Similarly,

$$
\begin{aligned}
& M_{12}=\left\langle\overline{\mathrm{K}}^{\mathrm{0}}\left|\mathscr{H}_{\mathrm{wk}}\right| \mathrm{K}^{\mathrm{o}}\right\rangle+ \\
& +\sum_{n}\left\{\left\langle\overline{\mathrm{K}}^{\mathrm{o}}\left|\mathscr{K}_{\mathrm{wk}}\right| n\right\rangle\left\langle n\left|\mathscr{H}_{\mathrm{wk}}\right| \mathrm{K}^{\mathrm{o}}\right\rangle\left(\mathrm{m}_{\left.\mathrm{K}^{\mathrm{o}}-\mathrm{m}_{n}\right)}\right\}\right.
\end{aligned}
$$

where the sum extends over all possible states $n$.

There are four possible sources of the observed symmetry invariance: $\operatorname{Im}\left(M_{12}\right), \operatorname{Im}\left(\Gamma_{12}\right),\left(\Gamma_{11}-\Gamma_{22}\right)$ and $\left(M_{11}-M_{22}\right)$. If one assumes CPT is a good symmetry then $\delta=0$ and only $\operatorname{Im}\left(M_{12}\right)$ and $\operatorname{Im}\left(\Gamma_{12}\right)$ contribute to $C P$ symmetry violation. There is considerable evidence that CPT is a good symmetry ${ }^{(12)}$. A consequence of CPT symmetry is that the mass, lifetime and the magnitude of the magnetic moment for a particle and its antiparticle are the same. The neutral kaon system provides the most sensitive test of CPT due to the small mass difference between the short and long-lived $\mathrm{K}^{0}$ states, 


$$
\left(m_{K}-m_{\bar{K}}\right) / m_{K} \leq\left(m_{\ell}-m_{S}\right) / m_{K} \approx 0.7 \times 10^{-14}
$$

implying $m_{\bar{K}} \approx m_{\mathrm{K}}$ to a high degree of accuracy.

Contributions to $\mathrm{CP}$ symmetry violation through $\operatorname{Im}\left(\mathrm{M}_{12}\right)$ correspond to $\mathrm{K}^{0}$. $\overline{\mathrm{K}}^{\circ}$ mixing in flight and is independent of the kaon decay mode. On the other hand a complex $\Gamma_{12}$ affects the kaon's decay modes without changing the kaons in free flight. Most theories agree on the amount of $\mathrm{CP}$ symmetry violation due to $\operatorname{Im}\left(\mathrm{M}_{12}\right)$ since it is well measured experimentally, but differ on how much $\Gamma_{12} \mathrm{CP}$ symmetry violation occurs.

\subsubsection{CP Symmetry Violation in the $\pi \pi$ Channel}

CP symmetry violation in $\mathrm{K} \rightarrow \pi^{+} \pi^{-}$is well-measured and directly related to CP symmetry violation in $\mathrm{K} \rightarrow \pi^{+} \pi^{-} \pi^{0}$. To determine the source of the violation, the mass matrix or the decay matrix, one projects the $\mathrm{K}^{\circ}$ and $\overline{\mathrm{K}^{\circ}}$ states onto the $\pi \pi$ isospin base:

$$
\begin{aligned}
& \left\langle\pi \pi(\mathrm{I}=0)|\mathrm{T}| \mathrm{K}^{\mathrm{o}}\right\rangle=\mathrm{A}_{\mathrm{o}} \mathrm{e}^{i \delta_{\mathrm{o}}} \\
& \left\langle\pi \pi(\mathrm{I}=2)|\mathrm{T}| \mathrm{K}^{\mathrm{o}}\right\rangle=\mathrm{A}_{2} \mathrm{e}^{i \delta_{2}} \\
& \left\langle\pi \pi(\mathrm{I}=0)|\mathrm{T}| \overline{\mathrm{K}}^{\mathrm{o}}\right\rangle=\overline{\mathrm{A}}_{\mathrm{o}} \mathrm{e}^{i \delta_{\mathrm{o}}} \\
& \left\langle\pi \pi(\mathrm{I}=2)|\mathrm{T}| \overline{\mathrm{K}}^{\mathrm{o}}\right\rangle=\overline{\mathrm{A}}_{2} \mathrm{e}^{i \delta_{2}}
\end{aligned}
$$

where $\delta_{0}$ and $\delta_{2}$ are the $\pi \pi$ scattering phase shifts. The only allowed modes of isospin are 0 and 2 because of the pseudoscalar character of $K$ and $\pi$.

The measurable decay amplitudes are:

$$
\eta_{+-}=\frac{\left\langle\pi^{+} \pi^{-}|\mathrm{T}| \mathrm{K}_{\ell}^{\mathrm{o}}\right\rangle}{\left\langle\pi^{+} \pi^{-}|\mathrm{T}| \mathrm{K}_{\mathrm{s}}^{\mathrm{o}}\right\rangle} \text { and } \eta_{o o}=\frac{\left\langle\pi^{\mathrm{o}} \pi^{\mathrm{o}}|\mathrm{T}| \mathrm{K}_{\ell}^{\mathrm{o}}\right\rangle}{\left\langle\pi^{0} \pi^{\mathrm{o}}|\mathrm{T}| \mathrm{K}_{\mathrm{s}}^{\mathrm{o}}\right\rangle}
$$

The $M_{12}$ contribution to these amplitudes is the same whereas the $\Gamma_{12}$ contributions differ due to the difference in the decay modes.

With CPT invariance $\overline{\mathrm{A}}_{\mathrm{o}}=-\mathrm{A}_{\mathrm{o}}{ }^{*}$ and $\overline{\mathrm{A}}_{2}=-\mathrm{A}_{2}{ }^{*}$ if one defines CPT $\left|\overline{\mathrm{K}}^{0}\right\rangle=$ $-\left|K^{0}\right\rangle$. Using the definitions of $K_{s}$ and $K_{\ell}$ given in equations 1.10 and 1.11 and 
assuming CPT invariance one finds:

$$
\begin{aligned}
& \eta_{+-}=\epsilon+i t_{0}+i \omega / \sqrt{2}\left(t_{2}-t_{0}\right) \\
& \eta_{00}=\epsilon+i t_{0}-i \sqrt{2} \omega\left(t_{2}-t_{0}\right)
\end{aligned}
$$

where:

$$
\begin{gathered}
t_{0}=\operatorname{Im}\left(A_{0}\right) / \operatorname{Re}\left(A_{0}\right), t_{2}=\operatorname{Im}\left(A_{2}\right) / \operatorname{Re}\left(A_{2}\right), \\
\omega=\operatorname{Re}\left(A_{2}\right) / \operatorname{Re}\left(A_{0}\right) e^{i\left(\delta_{2}-\delta_{0}\right) .}
\end{gathered}
$$

$\mathrm{CP}$ and CPT invariance in the decays implies that the amplitudes $A_{0}$ and $A_{2}$ are real making $t_{2}=t_{0}=0$. In this case:

$$
\eta_{+-}=\eta_{00}=\epsilon,
$$

and all $\mathrm{CP}$ symmetry violation occurs in the mass mixing matrix.

The parameter $\epsilon^{\prime}$ was introduced to define the decay matrix CP symmetry violating portion of $\eta_{+-}$and $\eta_{00^{\circ}}$. The relative phase of $\mathrm{K}^{\circ}$ and $\overline{\mathrm{K}^{\circ}}$ can be defined such that one of the two quantities $A_{0}$ or $A_{2}$ are real. This lead to two slightly different definitions of $\epsilon^{\prime}$ due to phase conventions.

Wu and Yang define the phase such that $A_{0}$ is real, $t_{0}=0$ and:

$$
\begin{aligned}
& \eta_{+-}=\epsilon+i / \sqrt{2}\left(\operatorname{Im}\left(\mathrm{A}_{2}\right) / \mathrm{A}_{\mathrm{o}}\right) \mathrm{e}^{i\left(\delta_{2}-\delta_{0}\right)} \\
& \eta_{00}=\epsilon-2 i / \sqrt{2}\left(\operatorname{Im}\left(\mathrm{A}_{2}\right) / \mathrm{A}_{\mathrm{o}}\right) \mathrm{e}^{i\left(\delta_{2}-\delta_{0}\right)}
\end{aligned}
$$

with:

$$
\epsilon^{\prime}=i / \sqrt{2}\left(\operatorname{Im}\left(\mathrm{A}_{2}\right) / \mathrm{A}_{\mathrm{o}}\right) \mathrm{e}^{i\left(\delta_{2}-\delta_{0}\right)}
$$

one obtains:

$$
\begin{aligned}
& \eta_{+-}=\epsilon+\epsilon^{\prime} \\
& \eta_{00}=\epsilon-2 \epsilon^{\prime}
\end{aligned}
$$

The Kobayashi-Maskawa $(K-M)$ phase convention has $A_{2}$ real, $t_{2}=0$ and $A_{0}$ has phase $\xi$.

$$
\begin{aligned}
& \eta_{+-}=1 / \sqrt{2} \mathrm{e}^{i(\pi / 4)}\left(\epsilon_{m}+2 \xi\right)+\epsilon^{\prime} \\
& \eta_{00}=1 / \sqrt{2} \mathrm{e}^{i(\pi / 4)}\left(\epsilon_{m}+2 \xi\right)-2 \epsilon^{\prime}
\end{aligned}
$$

where:

$$
\epsilon_{m}=\operatorname{Im}\left(\mathrm{M}_{12}\right) / \operatorname{Re}\left(\mathrm{M}_{12}\right) \quad \text { calculated in the "quark basis" }
$$

and:

$$
\epsilon^{\prime}=-i \xi / \sqrt{2}\left[\operatorname{Re}\left(\mathrm{A}_{2}\right) / \operatorname{Re}\left(\mathrm{A}_{\mathrm{o}}\right)\right] \mathrm{e}^{i\left(\delta_{2}-\delta_{0}\right)}=-i \xi \omega / \sqrt{2}
$$


Similarly for the $\mathrm{K} \rightarrow \pi \pi \pi$ mode one can define $\eta_{+-0}$ and $\epsilon_{+-0}^{\prime}$ :

$$
\begin{aligned}
& \eta_{+-\mathrm{o}}=\frac{\left\langle\pi^{+} \pi^{-} \pi^{0}|\mathrm{~T}| \mathrm{K}_{s}^{\mathrm{o}}\right\rangle}{\left\langle\pi^{+} \pi^{-} \pi^{0}|\mathrm{~T}| \mathrm{K}_{\ell}^{\mathrm{o}}\right\rangle}=\epsilon+\epsilon_{+-0}^{\prime} \\
& \epsilon_{+-o}^{\prime}=i\left[\frac{\operatorname{Im} A\left(\mathrm{~K}^{\circ} \rightarrow \pi^{+} \pi^{-} \pi^{o}\right)}{\operatorname{Re} A\left(\mathrm{~K}^{0} \rightarrow \pi^{+} \pi^{-} \pi^{0}\right)}-\frac{\operatorname{Im} \mathrm{A}_{0}}{\operatorname{Re} \mathrm{A}_{\mathrm{o}}}\right]
\end{aligned}
$$

Here $\epsilon$ is the same as that for the two-pion modes since it is from the kaon mass matrix, and $\epsilon_{+-0}^{\prime}$ comes from the element $\Gamma_{12}$ in the three pion decay matrix where $A_{0}$ is defined similarly to $A_{0}$ in $K^{0} \rightarrow 2 \pi$. There are two general patterns for models describing $\mathrm{K} \rightarrow 3 \pi^{(13)}$. Both require a $\Delta \mathrm{I}=3 / 2$ transition. In one the $\Delta \mathrm{I}=3 / 2$ transition is supressed which implies $\epsilon_{+-0}^{\prime} \approx \epsilon^{\prime}$. In the other case there is no $\Delta \mathrm{I}=3 / 2$ suppression and $\epsilon_{+-0}^{\prime} \approx 22 \epsilon^{\prime}$. Most models are closer to the first case in their predictions.

\subsection{Theoretical Models}

It is possible to incorporate $\mathrm{CP}$ symmetry violation into the standard Weinberg-Salam electroweak gauge theory by adding a third generation of quarks as first pointed out by Kobayashi and Maskawa ${ }^{(14)}$. Alternatives to the KobayashiMaskawa (K-M) model require an enlargement of the gauge group or an expansion of the Higgs sector. All these models provide new mechanisms for CP symmetry violation in $\mathrm{K}^{\mathrm{O}}$ decays, but many of these mechanisms supplement rather than replace the $\mathrm{K}-\mathrm{M}$ mechanisms.

A large amount of theoretical work has been done on CP symmetry violation in the $\mathrm{K}->3 \pi$ modes, most using the standard model with three generations of quark doublets. In order to agree with experimental results, all viable models must predict $\epsilon \sim 10^{-3}$. The four models I will discuss do predict this value of $\epsilon$, although the Left-Right Symmetric and the Weinberg-Three Higgs models have variations in the calculation of $\epsilon$. All models differ in the decay matrix calculation of $\Gamma_{12}$ corresponding to $\epsilon_{+-}^{\prime} \cdot$. Results are usually reported in terms of differences between 
$\epsilon_{+-}^{\prime}$ and $\epsilon_{+-0}^{\prime}$

The "superweak" model of Wolfenstein ${ }^{(15)}$ was one of the first proposed for CP symmetry violation and is the only model not based on the standard three generation model. It predicts $\epsilon$ is zero which may or may not be consistent with the latest experimental results for $\epsilon^{\prime} / \epsilon$. Other models, one involving more Higgs particles and another a right-handed $\mathrm{W}$ particle need more theoretical work as these calculations have large variations. I will go into more depth with the K-M model since most theoretical work has been done based on this model.

\subsubsection{The Kobayashi-Maskawa Model}

In the electroweak theory, the charged weak quark currents are flavor mixed. The mixing matrix is unitary. For two generations there is a single mixing angle and the currents are $\mathrm{CP}$ conserving. In the case of the three generation model, the unitarity mixing matrix has three mixing angles and one complex phase which can give rise to $\mathrm{CP}$ violation. In 1973, before the third generation had been discovered, Kobayashi and Maskawa proposed this theory for $\mathrm{CP}$ symmetry violation(14). CP symmetry violation is introduced by complex Yukawa couplings appearing in the mass terms of the Lagrangian after spontaneous symmetry breaking. Diagonalization of the quark mass matix leads to charged current interactions which are nondiagonal in quark flavor eigenstates. The weak hadronic current is:

$$
\mathrm{J}_{\mu}=(\overline{\mathrm{u}}, \overline{\mathrm{c}}, \overline{\mathrm{t}}) \gamma_{\mu}\left(1-\gamma_{5}\right) \mathrm{U}_{\mathrm{KM}}(\mathrm{d}, \mathrm{s}, \mathrm{b})^{\top}
$$

with the K-M matrix:

$$
\mathrm{U}_{\mathrm{KM}}=\left(\begin{array}{lll}
\mathrm{U}_{u d} & \mathrm{U}_{u s} & \mathrm{U}_{u b} \\
\mathrm{U}_{c d} & \mathrm{U}_{c s}^{\delta} & \mathrm{U}_{c b}^{\delta} \\
\mathrm{U}_{t d} & \mathrm{U}_{t s}^{\delta} & \mathrm{U}_{t b}^{\delta}
\end{array}\right)
$$

For three or more generations, these currents in general can introduce $\mathrm{CP}$ symmetry violating processes. All the $\mathrm{U}_{\mathrm{ij}}$ elements are functions of three quark mixing angles, and the $\mathrm{U}_{\mathrm{ij}} \delta$ elements are also functions of the complex CP symmetry violating phase " $\delta$ " (not the $\delta$ of equation 1.13 ) which cannot be transformed away by a redefinition of the phases of the quark fields. 
For $\delta$ non-zero (or $2 \pi$ ), imaginary terms in the $U_{i j}^{\delta}$ coupling of $s \rightarrow c, s \rightarrow t$ and $b \rightarrow c, b \rightarrow t$ will result in $C P$ violation. The $s \rightarrow c$ and $s \rightarrow t$ imaginary terms account for $\mathrm{CP}$ symmetry violation in the $\mathrm{K}$-system. But $\mathrm{b} \rightarrow \mathrm{c}$ and $\mathrm{b} \rightarrow \mathrm{t}$ imaginary terms predict CP symmetry violation in the B-system also. This is yet to be observed.

There are several parameterizations of the $\mathrm{K}-\mathrm{M}$ matrix. $\mathrm{K}-\mathrm{M}$ use

$$
\mathrm{U}_{\mathrm{KM}}=\left(\begin{array}{ccc}
c_{1} & -s_{1} c_{3} & -s_{1} s_{3} \\
s_{1} c_{2} & c_{1} c_{2} c_{3}-s_{2} s_{3} e^{i \delta} & c_{1} c_{2} s_{3}+s_{2} c_{3} e^{i \delta} \\
s_{1} s_{2} & c_{1} s_{2} c_{3}+c_{2} s_{3} e^{i \delta} & c_{1} s_{2} s_{3}-c_{2} c_{3} e^{i \delta}
\end{array}\right)
$$

where $s_{i}=\sin \theta_{i}, c_{i}=\cos \theta_{i}$, choosing to make the first row and column of $\mathrm{U}_{\mathrm{KM}}$ real.

Several other useful parameterizations that exist ${ }^{(16)}$ easily incorporate B meson decays or expand the matrix elements in terms of $\lambda=\sin \theta_{c} \approx 0.22$.

Calculating $\epsilon, \epsilon^{\prime}$ and $\delta$ in $U$ is a difficult task. The main formulas are sensitive functions of quantities which still carry considerable theoretical uncertainties. The two major uncertainties are the hadronic matrix elements of the weak current $\otimes$ current operators and the short-distance expansions computed by methods of Quantum Chromodynamics.

In the observed $\mathrm{CP}$ nonconserving decay $\mathrm{K}_{\ell} \rightarrow \pi^{+} \pi^{-}$there are two possible sources of CP symmetry violation:

(1) the $\mathrm{K}^{0}-\overline{\mathrm{K}^{\mathrm{O}}}$ mass matrix $\mathrm{M}_{12}$

(2) the direct $\mathrm{K}_{\ell} \rightarrow \pi^{+} \pi^{-}$transition to pions

The same is true for $\mathrm{K}_{\mathrm{s}} \rightarrow \pi^{+} \pi^{-} \pi^{\circ}$. The CP parameter $\epsilon$ receives contributions from both sources while $\epsilon^{\prime}$ comes uniquely from direct transitions. The standard model Feynman diagrams which induce CP symmetry violation in the mass matrix are shown in Figure 1.1. The double penguin and the Siamese penguin contributions are smaller than the box diagram(17). The effect of the dispersive diagram is calculated using chiral symmetry and is found to be a minor contribution to $\epsilon^{(13)}$. 


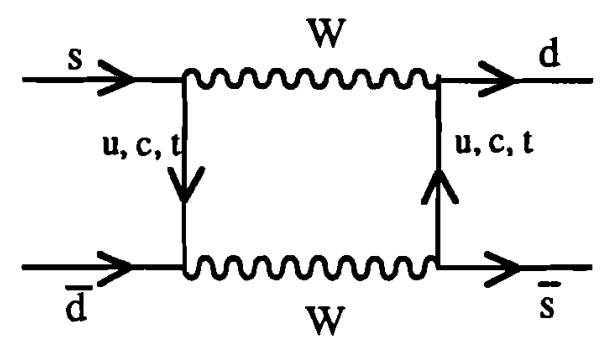

(a) Box Diagram

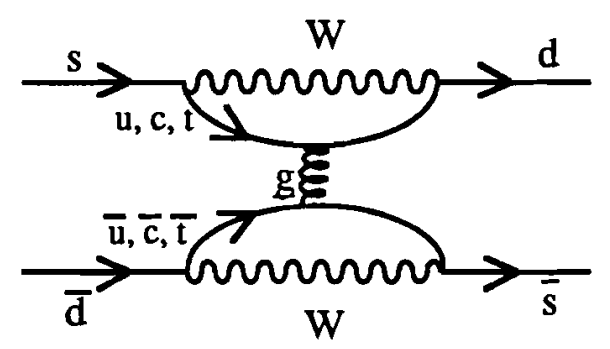

(c) Siamese Penguin

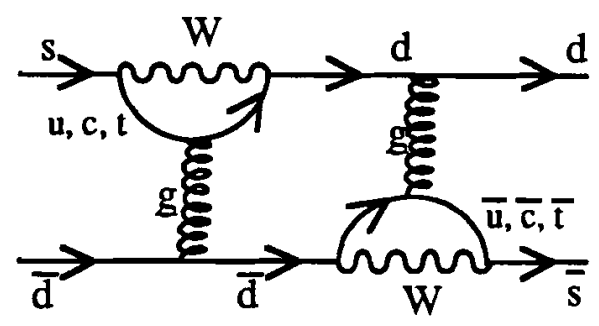

(b) Double Penguin

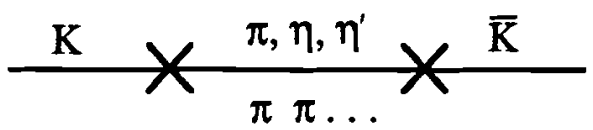

(d) Dispersive Diagram

Flgure 1.1 Mass Matrlx Feynman Dlagrams

The contributions to the decay matrix, or $\epsilon$, are from low-mass states into which the $\mathrm{K}^{\mathrm{O}}$-mesons decay. The matrix elements correspond to "penguin" diagrams shown in Figure 1.2, which are $\Delta s=1$ transitions.

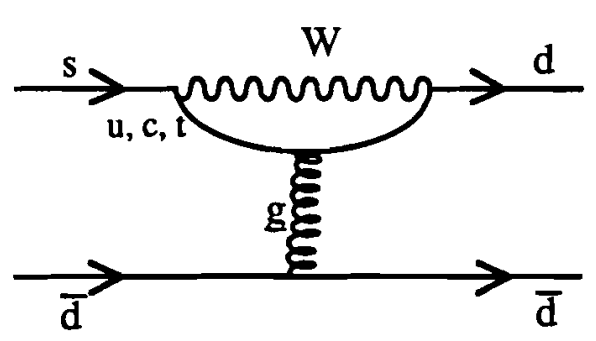

(a) Penguin Diagram

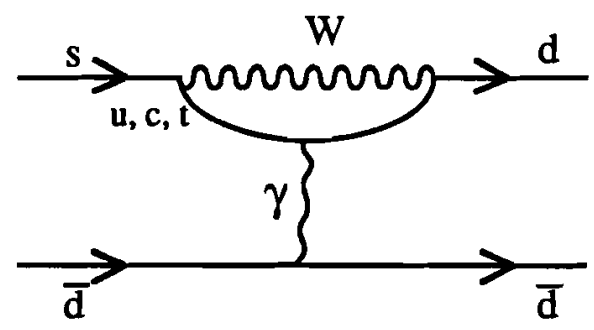

(b) Electromagnetic Penguin

\section{Flgure 1.2 Decay Matrlx Feynman Dlagrams}

The matrix elements can be estimated by a variety of methods. The results have large uncertainties due to the lack of knowledge concerning the amplitudes and relative phases of the decays. In $\mathrm{K} \rightarrow 2 \pi$ decay the electromagnetic penguin and isospin breaking generate a phase in $A_{2}$ of equation 1.17 which is enhanced by $a$ factor of 22 over any phase contributions in $\mathrm{A}_{\mathrm{o}}$. A standard parametrization yields: 


$$
\begin{gathered}
\frac{\epsilon^{\prime}}{\epsilon}=\frac{-i}{22 \sqrt{2}} e^{i\left(\delta_{2}-\delta_{0}\right)} \frac{\operatorname{Im} A_{0}}{\operatorname{Re} A_{0}}(1-\Omega) \\
\Omega=22\left(\frac{\operatorname{Im} A_{2}}{\operatorname{Im} A_{0}}\right)
\end{gathered}
$$

Calculations by L. Wolfenstein ${ }^{(18)}$ predict:

$$
7.0 \times 10^{-3}>\left|\frac{\epsilon^{\prime}}{\epsilon}\right|>1.0 \times 10^{-3}
$$

For $\mathrm{K} \rightarrow 3 \pi$ decay higher order chiral Lagrangians possibly play an important role. At lowest order chiral symmetry, where $A_{0}$ is real, then all $\Delta I=1 / 2, K \rightarrow 3 \pi$ amplitudes will be real also. This allows only $\Delta \mathrm{I}=3 / 2 \mathrm{CP}$ symmetry violation yielding the $\mathrm{Li}$ and Wolfenstein ${ }^{(19)}$ result:

$$
\begin{gathered}
\left|\eta_{+-0^{-}} \epsilon\right|=2 / 3\left|\eta_{+-}-\eta_{o 0}\right| \\
\left|\epsilon_{+-0}^{\prime}\right|=2\left|\epsilon_{+-}^{\prime}\right|
\end{gathered}
$$

at the center of the Dalitz plot. And if $s_{1}=s_{2}$ :

$$
\eta_{+-o}-\eta_{+-}=-\left(\eta_{+-}-\eta_{o o}\right)\left(1-\frac{5}{2} \frac{s_{3}-s_{0}}{m_{\mathrm{K}}^{2}-m_{\pi}^{2}}\right)
$$

where $s_{i}$ are the Lorentz invariant center of mass frame kinetic energies:

$$
\begin{gathered}
s_{i}=\left(\mathrm{P}_{\mathrm{K}}-\mathrm{P}_{\pi_{i}}\right) \\
\mathrm{s}_{\mathrm{o}}=\left(\mathrm{s}_{1}+\mathrm{s}_{2}+\mathrm{s}_{3}\right) / 3=\mathrm{m}_{\pi}^{2}+1 / 3 \mathrm{~m}_{\mathrm{K}}
\end{gathered}
$$

Here $P$ is the momentum and the index 3 refers to the neutral pion. For $s_{1} \neq s_{2}$ the situation is complicated by the CP symmetry conserving decay into 3 pions. This is discussed in Appendix E.

If one includes photopenguin diagrams and $\pi-\eta$ mixing in the calculation of $\epsilon^{\prime}{ }_{+-0}$ one can obtain different results. Electromagnetic penguin diagrams and particle mixing can enhance $\epsilon_{+-0}^{\prime}$ by 2 to 4 times $\epsilon^{\prime}$. At higher order, quadratic terms can generate $\epsilon_{+-0}^{\prime}$ without $\Delta \mathrm{I}=3 / 2$ suppression ${ }^{(20)}$ yielding ratios of $\epsilon^{\prime}{ }_{+-0} / \epsilon^{\prime}$ up to 20 . A more realistic estimate is: 


$$
\epsilon_{+-o}^{\prime} \sim 0\left[\frac{m_{K}^{2}}{\Lambda^{2}} * 22 \epsilon^{\prime}\right] \rightarrow 10 \epsilon^{\prime} \quad(13)
$$

Here the factor of $22 \epsilon^{\prime}$ is the effect of direct $C P$ symmetry violating phase and the $\Lambda^{2}$ term indicates the chiral suppresion since $\Lambda$ is the chiral scale parameter expected to be $\sim 1 \mathrm{GeV}$. Donoghue advises that this result should not be used as a definite prediction but as an illustration of how one might get large values of $\epsilon_{+-0}^{\prime} / \epsilon^{\prime}$. And indeed Fajfer and Gerard(21) disagree with the enhancement effects of isospinbreaking and higher-order chiral expansion terms. They predict:

$$
\epsilon_{+-0}^{\prime}=(1.3 \pm 0.7) \epsilon^{\prime}
$$

\subsubsection{The Superweak Model}

L. Wolfenstein ${ }^{(15)}$ proposed the "superweak" model where CP symmetry violation is due to a $\Delta S=2$ transition only, corresponding to the second order term in the standard model perturbative expansion shown in Figure 1.1. He proposed a new interaction $\mathscr{X}_{\mathrm{sw}}^{\prime}$ which changes only the $\mathrm{M}_{12}$ portion of the Hamiltonian in order to make CP symmetry violation possible in the two quark family model. This is why the term "superweak" CP symmetry violation is used when referring to C P syrrmetry violation in $\mathrm{K}^{\circ}-\overline{\mathrm{K}}^{\circ}$ mixing. $\mathrm{CP}$ non-invariance in this model is the same for all decay modes since it occurs only in the mass matrix, consistent with CCFT experimental results and all results since then with the possible exception of the latest NA31 result.

In the superweak model the Hamiltonian is :

$$
\mathscr{H}_{\text {w }}=\alpha \mathscr{X}^{1}{ }_{\text {wk }}+\beta \mathscr{H}_{\text {sw }}^{\prime}
$$

where $\mathscr{X}^{1}{ }_{\mathrm{wk}}$ is the normal weak interaction with $\Delta s=1$ and $\mathrm{CP}$ invariant, and $\mathscr{H}^{\prime}{ }_{s w}$ is $\Delta s=2$ and $\mathrm{CP}$ non-invariant. Here the violation is due to the interference term between the first-order superweak interaction and the second-order Fermi interaction. The ratio $\alpha / \beta$ is fixed since the amplitude of the violation is of order $\epsilon$.

$$
\begin{gathered}
\epsilon \approx\left(F_{\mathrm{Sw}}\right) /\left(\mathrm{G}^{2}\right)=\beta / \alpha \times 10^{5} \\
\beta / \alpha=10^{-7}
\end{gathered}
$$

Substituting these values into equations 1.14 and 1.15 in order to obtain $M_{i j}$ 
and $\Gamma_{i j}$ one finds:

$$
\begin{aligned}
& \operatorname{Im} \mathrm{M}_{12} \propto \beta\left\langle\mathrm{K}^{0}\left|\mathscr{H}_{\mathrm{sw}}\right| \overline{\mathrm{K}}^{0}\right\rangle \\
& \operatorname{Re} \mathrm{M}_{12} \propto \alpha^{2} \\
& \operatorname{Im} \Gamma_{12}=0 \\
& \operatorname{Re} \Gamma_{12} \propto \alpha^{2}
\end{aligned}
$$

Since $\operatorname{Im}\left(\Gamma_{12}\right)=0, \epsilon^{\prime}=0$ and there is no direct (decay) CP symmetry violation. Then:

$$
\begin{aligned}
& \eta_{+-0}=\eta_{000}=\eta_{+-}=\eta_{00}=\epsilon \\
& \varphi_{+-0}=\varphi_{000}=\varphi_{+-}=\varphi_{00}=\varphi_{n}
\end{aligned}
$$

where $\varphi$ is the angle of $\eta$ in the complex plane. Using equation 1.12 for $\epsilon$ one finds:

$$
\epsilon_{s w}=\frac{-\operatorname{Im} M_{12}}{i\left(m_{s}-m_{\ell}\right)+\left(\gamma_{s}-\gamma_{\ell}\right) / 2}
$$

This can then be solved for $\operatorname{Im}\left(\mathrm{M}_{12}\right)$ using the experimental values for $\left(m_{\mathrm{s}}-m_{\ell}\right)$, $\gamma_{s}, \gamma_{l}$ and $\epsilon$ yielding $\operatorname{Im}\left(\mathrm{M}_{12}\right)=1.16 \times 10^{-8} \mathrm{eV}$. Until 1989 this was the status of $\mathrm{CP}$ symmetry violation as no direct $\mathrm{CP}$ symmetry violation had been observed. Very recently $C P$ violation of the direct type has been reported from the CERN experiment NA-31. They have preliminarily measured $\epsilon^{\prime} \epsilon=(3.3 \pm 1.1) \times 10^{-3(32)}$. A non-zero measurement of $\epsilon^{\prime} / \epsilon$ conflicts with the predictions of the Superweak Model. The CERN result apparently disagrees with the result from FNAL experimental $E 731$ which measures $\epsilon^{\prime} / \epsilon=(-0.4 \pm 1.4$ (stat $) \pm 0.6($ syst $\left.)\right) \times 10^{-3}$ (22).

\subsubsection{The Weinberg-Higgs Model}

Weinberg ${ }^{(23)}$ suggested a model of $\mathrm{CP}$ symmetry violation based on the standard model, by which flavor-changing neutral Higgs couplings were forbidden by a discrete symmetry, but $\mathrm{CP}$ symmetry violation still occurred as a result of the couplings among three Higgs boson fields. Here CP non-invariance could arise 
spontaneously. The major $\mathrm{CP}$ symmetry violating effect was identified as a phase in the mixing matrix that diagonalizes the charged Higgs mass matrix (24).

Like $\mathrm{W}^{+}$, the charged Higgs boson exchanges cause a flavor change $\Delta \mathrm{s}=1$. Calculations involve diagrams like Figure 1.1 and 1.2 where one or both W's are replaced by a charge Higgs boson. Two estimates of $\epsilon^{\prime} / \epsilon$ are:

$$
\epsilon^{\prime} / \epsilon=-0.006^{(25)} \text { and } \epsilon^{\prime} / \epsilon=-0.016^{(26)} \text {, }
$$

but both have large uncertainties.

For $\mathrm{K}^{\mathrm{O}}>3 \pi$, H. Y. Cheng ${ }^{(27)}$ predicts, at the center of the Dalitz plot:

$$
\left|\eta_{+-o}-\epsilon\right|=2\left|\epsilon^{\prime}\right| \text {. }
$$

The model can be viable if dispersive effects in $\mathrm{K}^{0}-\overline{\mathrm{K}}^{\mathrm{o}}$ mixing provide the major contributions in the kaon sector (24) for a non-zero $\epsilon^{\prime}$.

\subsubsection{The Left-Right Symmetric Model}

The Left-Right Symmetric model is also based on the standard model, but enlarges the gauge group to allow $\mathrm{CP}$ symmetry violation. One of the simplest ways to enlarge the gauge group is to add an $\mathrm{SU}(2)_{\mathrm{R}}$ gauge interaction mediated by bosons $\mathrm{W}_{R}$ that couple to right-handed currents. The gauge group is labelled $S U_{L}(2) X$ $S U_{R}(2) \times U(1)$. SU(2) $)_{L}$ is the $S U(2)$ associated with the usual bosons $\mathrm{W}^{ \pm}$.

It is generally assumed that parity is a spontaneously broken symmetry in this model and is restored for energies well above the mass of $W_{R}$. This model has been extensively reviewed by Mohapatra and others ${ }^{(28)}$. Mohapatra and Pati(29) pointed out that with two generations of quarks this model could allow CP symmetry violation. $\mathrm{CP}$ symmetry violation occurs once $\mathrm{W}_{\mathrm{R}}$ exchanges are included since the right-handed portion of the K-M matrix, $U_{R}$, contains complex elements if $U_{K M}$ is real. This model predicts:

$$
\eta_{+-}=\eta_{00} \text { and } \eta_{+-0}=\eta_{000}
$$

But $\eta_{+-0} \neq \eta_{+-}$, instead,

$$
\left(\eta_{+-}-\eta_{+-0}\right) \approx i\left|\eta_{+-}\right|
$$

According to Cheng(27), a more detailed analysis predicts:

$$
\frac{\left|\eta_{+-o}-\eta_{+-}\right|}{\left|\eta_{+-}\right|} \approx 10^{-2}
$$

He states that this result remains valid in order of magnitude even in the 
presence of a third quark generation.

\subsection{Experimental Values}

Until recently all measurements of $\epsilon^{\prime} / \epsilon$ were consistent with zero showing no evidence of CP symmetry violation in the decay modes. Two published results on experimental measurements of $\epsilon^{\prime} / \epsilon$ in 1985 were:

$$
\begin{aligned}
& \epsilon^{\prime} / \epsilon=0.002 \pm 0.007 \pm 0.002 \\
& \epsilon^{\prime} / \epsilon=-0.005 \pm 0.005 \pm 0.002
\end{aligned}
$$

This averages to $\epsilon^{\prime} / \epsilon=-0.003 \pm 0.005$.

Two recent measurements disagree. That from NA31 at CERN is:

$$
\epsilon^{\prime} / \epsilon=0.0033 \pm 0.0011
$$

This is 3 standard deviations away from zero and well within the predictions of the standard $\mathrm{K}-\mathrm{M}$ Model if the top mass is between 50 and $100 \mathrm{GeV}^{(33)}$. The non-zero value tends to cast some doubt on the superweak theory which predicts $\epsilon^{\prime} / \epsilon$ to be zero. On the other hand, the result from E731 at Fermilab is consistent with both zero and the standard model:

$$
\epsilon^{\prime} / \epsilon=-0.0004 \pm 0.0014 \pm 0.0006
$$

If one uses the results from NA31 and the Particle Data Group ${ }^{(34)}$ value of $\epsilon=$ 0.0023 , one can determine $\epsilon^{\prime} \approx 7.0 \times 10^{-6}$. The standard model predicts $1 \times 10^{-3}<$ $\epsilon^{\prime} / \epsilon<7 \times 10^{-3}$ or $10^{-6}<\epsilon^{\prime}<7 \times 10^{-6}$. Recall:

$$
\eta_{+-0}=\epsilon+\epsilon_{+-0}^{\prime}
$$

All theories predict $\epsilon \sim 10^{-3}$. Theoretically the largest value of $\epsilon_{+-0}^{\prime} \sim 20 \epsilon_{+-}^{\prime}$ or $\sim 1-2 \times 10^{-4}$, although this value is most likely high an order of magnitude. Thus $\eta_{+-0}$ is dominated by $\epsilon$ and of order $10^{-3}$. All theories also predict $\operatorname{Re}\left\{\eta_{+-0}\right\} \approx \operatorname{Re}\left\{\eta_{+}\right\}$ $=0.0016$ (experimentally measured value). This is because the major contribution to $\operatorname{Re}\left\{\eta_{+-0}\right\}$, (or $\operatorname{Re}\left\{\eta_{+}\right\}$for that matter), is through the mass matrix term and thus the contribution to $\operatorname{Re}\left\{\eta_{+-0}\right\}$ is very nearly equal to that measured for $\operatorname{Re}\left\{\eta_{+}\right\}$since both measurements are for kaons. For this reason many $\eta_{+-0}$ experiments measure $\operatorname{Im}\left\{\eta_{+-0}\right\}$ constraining $\operatorname{Re}\left\{\eta_{+-0}\right\}$ to 0 (approximately 0.0016 for their statistics). Experimentally the measurement of $\operatorname{Im}\left\{\eta_{+-0}\right\}$ is the important value to obtain. 


\begin{tabular}{|c|c|c|c|c|c|}
\hline Year & Events & $\operatorname{Re}(\eta+-6$ & $\operatorname{Im}\left(\eta_{+-\delta}\right)$ & $\operatorname{Im}\left(\eta+\sigma^{*}\right.$ & Reference \\
\hline 1965 & 18 & $0.25 \pm 0.55$ & $0.08 \pm 0.55$ & & Anderson (1) \\
\hline$-\overline{1966}$ & $-\overline{136}$ & ---- & ---- & $-0 . \overline{34}+\overline{0} .1 \overline{9}$ & $\overline{B e h r} \overline{(2)}-$ \\
\hline$-\overline{1970}$ & $--\overline{53}$ & ---- & ---- & $-0 . \overline{45} \pm \frac{-0.59}{0.45}$ & $\overline{\text { Webber }} \overline{(3)}--$ \\
\hline$-\overline{1970}$ & $-\overline{7}_{1}$ & $-0 . \overline{05} \pm \overline{0} .3 \overline{0}$ & $-0 . \overline{1} 5 \pm \overline{0} .4 \overline{5}$ & $-0 \overline{25}+\overline{0} 4 \overline{0}$ & Webber $\overline{(3)}-$ \\
\hline--- & & ---- & ---- & -- & $=----$ \\
\hline 1971 & & $2.10+0.55$ & $0.09+0.40$ & & Meisner (4) \\
\hline$-\overline{1971}$ & $-\overline{99}$ & $-\frac{-0.60}{0.47 \pm 0.20}$ & $-0 . \overline{10} \pm 0.30$ & $-0 . \overline{6} 6+\overline{0} .2 \overline{7}$ & $\overline{C h o} \overline{(5)}-$ \\
\hline--- & -- & ---- & ---- & ---0.32 & \\
\hline 1971 & & $0.01+0.22$ & $0.33+0.42$ & & James (6) \\
\hline$\overline{1972}$ & $-\overline{180}$ & $-0 . \overline{17}+0.17$ & $-0 . \overline{0}+\frac{-0.50}{0.38}$ & $-0 . \overline{3} 1+\overline{0} .3 \overline{1}$ & James $(7)$ \\
\hline--- & -- & --0.23 & $--\underline{0.41}$ & $--\underline{-0.25}$ & \\
\hline 1972 & 99 & $-0.09 \pm 0.19$ & $0.56 \pm 0.43$ & & Jones (8) \\
\hline$-\overline{1972}$ & $-\overline{384}$ & $\overline{0} . \overline{13}+\overline{0.17} \overline{-}$ & $-\overline{0} \overline{17}+\overline{0.27}$ & $-\overline{0} \overline{05} \pm \overline{0} .2 \overline{0}$ & $\overline{\text { Metcalf }} \overline{(9)}-$ \\
\hline & & --0.20 & --0.26 & - - & \\
\hline 1973 & 148 & $-0.05 \pm 0.17$ & $0.39+0.35$ & & Mallary $\overline{(10)}$ \\
\hline$-\overline{1975}$ & $-\overline{192}$ & $-0 . \overline{04} \pm \overline{0} .1 \overline{8}$ & $0 . \overline{75}+0.30$ & $0 . \overline{75}+\overline{0} .2 \overline{7}$ & $-\overline{-}---\overline{1}$ \\
\hline-- & - - & & --0.35 & --0.35 & (11) \\
\hline 1985 & 409 & $-0.002 \pm 0.23$ & $-0.14 \pm 0.35$ & & Barmin (12) \\
\hline$\overline{1985}$ & $-\overline{601}$ & $-\overline{0} \overline{05} \pm \overline{0.17}$ & $\overline{0} . \overline{15} \pm \overline{0} .3 \overline{3}$ & --- & $\overline{\text { Barmin }}(\overline{12})--$ \\
\hline
\end{tabular}

Table 1.1 Experimental Values of $\eta_{+-0}($ References shown at the end of Chapter 1$)$ (NOTE: The listings for $\operatorname{Im}\left\{\eta_{+-o}\right\}^{*}$ are obtained by constraining $\operatorname{Re}\left\{\eta_{+-o}\right\}$ to be zero. The second Webber result is based on the first Webber result combined with Anderson's 18 events. Barmins second value is obtained using his 409 events combined with Baldo-Coelins 192 events. All results were obtained using an initially pure $\mathrm{K}^{\circ}$ or $\overline{\mathrm{K}^{\circ}}$ beam.)

The Particle Data Group has collected experimental limits on $\eta_{+-0}$ from many 
experiments. These values are recorded in Table 1.1 and the results for experiments after 1971 are plotted with their errors in Figure 1.3 and 1.4. Figure 1.5 shows the results for $\operatorname{Im}\left\{\eta_{+-0}\right\}$ when $\operatorname{Re}\left\{\eta_{+-0}\right\}$ is constrained to zero.

For this thesis, data from Fermilab experiment E621 were analyzed. The final data sample contained $122 \mathrm{~K}$ inclusively produced (i.e. $p+$ tungsten $-\mathrm{K}+\mathrm{X}$ where $\mathrm{X}$ is unknown) $\mathrm{K}->\pi^{+} \pi^{-} \pi^{\circ}$ events. Although the statistics of this experiment are not on the level of a $10^{-3}$ measurement, it is the largest sample of $\mathrm{K}^{0} \rightarrow 3 \pi$ decays ever measured in the proper time region of interest. With this data one can check to see if $\eta_{+-0}$ is on the order of $10^{-2}$ or less as all theories predict.

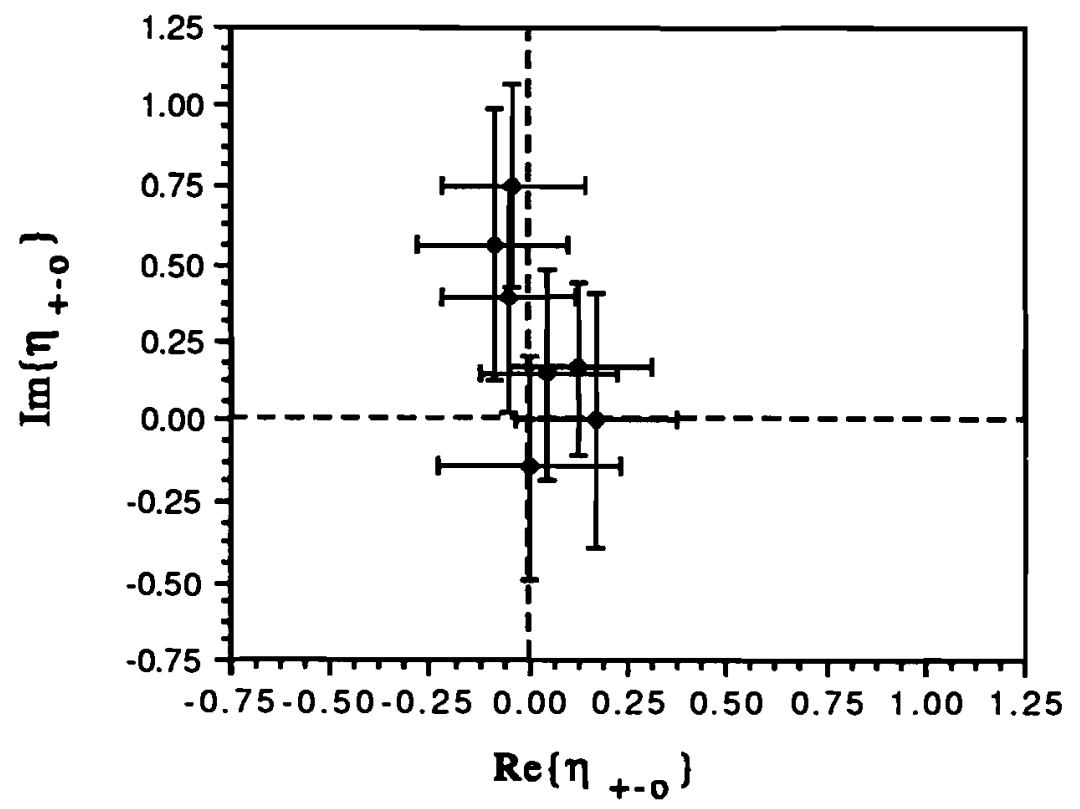

Figure 1.3 Graph of Experimental Values of $\eta_{+-0}$ from 1972 to Present 


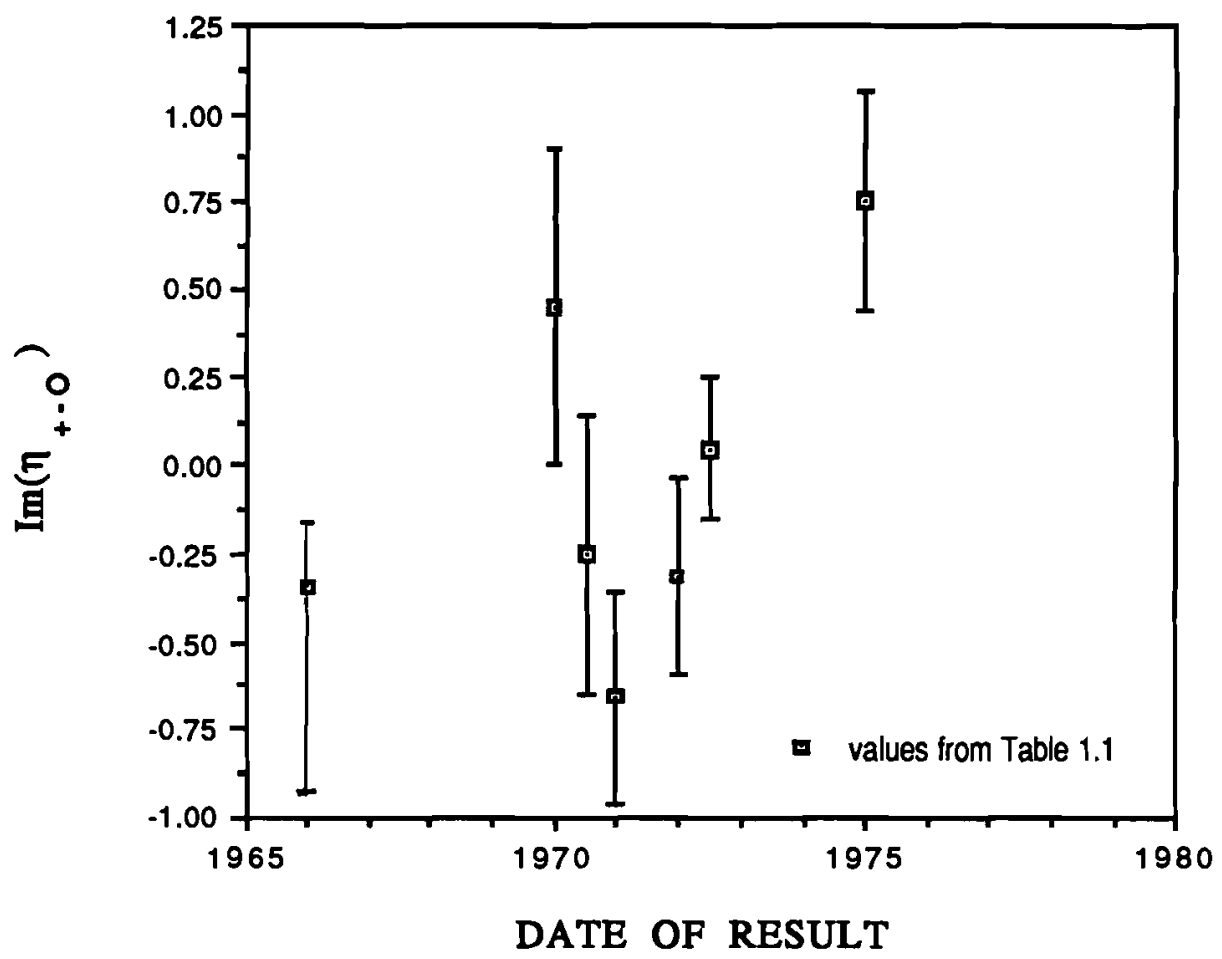

Figure 1.4 Experlmental Values of $\operatorname{Im}\left\{\eta_{+-0}\right\}$ for $R \theta\left[\eta_{+-0}\right\}$ Constralned to Zero

\subsection{Experimental Approach}

In the $\mathrm{K}^{\circ} \rightarrow \pi^{+} \pi^{-}$decay, the $\mathrm{CP}$ non-invariant portion of the state is longlived and the $\mathrm{CP}$ invariant portion is short-lived. Thus one need only look for $\mathrm{K}^{\circ} \rightarrow$ $\pi^{+} \pi^{-}$decays far beyond several $\mathrm{K}_{\mathrm{s}}$ lifetimes to find $\mathrm{CP}$ symmetry violation. For $\mathrm{K}^{\circ} \rightarrow \pi^{+} \pi^{-} \pi^{\circ}$ decays the situation is reversed. The CP invariant term is shortlived, on the order of the $\mathrm{K}_{\mathrm{S}}$ lifetime, relative to the $\mathrm{CP}$ non-invariant $\mathrm{K}_{\ell}{ }^{\circ} \rightarrow \pi^{+} \pi^{\text {- }}$ $\pi^{\circ}$ decay term. In the $\mathrm{K}^{\circ} \rightarrow 3 \pi$ decay the $\mathrm{CP}$ symmetry violating signal is dominated by the CP symmetry conserving $\mathrm{K}_{\ell}{ }^{\circ}$ decay and thus the experiment must be done as an interference measurement.

The proper time dependence of a kaon decaying into pions is not purely 
exponential due to quantum mechanical interference between the short- and longlived decays. The number of $\mathrm{K}^{\circ} \rightarrow 3 \pi$ decays at proper time, $\mathrm{t}$, is given by the expression:

$$
\begin{aligned}
& \frac{d \mathrm{~N}(\mathrm{t})}{d t}=\mathrm{N}_{K}(0) \mathrm{BR}_{3 \pi}\left\{e^{-t / \tau_{\ell}}\right. \\
& \left.+2 \mathrm{D}_{\mathrm{K} \overline{\mathrm{K}}}\left|\eta_{+-o}\right| \cos \left(\Delta m t+\varphi_{+-o}\right) e^{-t / 2\left(1 / \tau_{\mathrm{s}}+1 / \tau_{\ell}\right)}+\left|\eta_{+-o}\right|^{2} e^{-t / \tau_{\mathrm{s}}}\right\}
\end{aligned}
$$

where $N_{K}(0)$ is the number of kaons produced at the target, $B R_{3 \pi}$ is the $K^{0} \rightarrow \pi^{+} \pi^{-}$ $\pi^{\circ}$ branching ratio, $\tau_{s}$ and $\tau_{\ell}$ are the $K_{s}$ and $K_{\ell}$ lifetimes, $\Delta m$ is the $K_{\ell}-K_{s}$ mass difference and $\left|\eta_{+-0}\right|$ and $\varphi_{+-0}$ are the magnitude and phase of the CP symmetry violation. $\mathbf{D}_{\mathrm{KK}}$, the "dilution factor", also enters into equation 1.56. Since $\eta_{+-0}$ is small, the third term in 1.56 can be ignored. We measure the second term. The size of this interference term depends on whether the amplitudes for $K_{s}$ and $K_{\ell}$ were coherently produced or not. $\mathbf{D}_{\mathrm{KK}}$ is a measurement of the "coherence" since:

$$
D_{K \bar{K}}=\frac{K^{0}-\overline{K^{0}}}{K^{0}+\overline{K^{0}}}
$$

where and $\mathrm{K}^{\circ}$ are $\overline{\mathrm{K}^{\circ}}$ the kaon production fluxes at the target. The method used for determining $\mathbf{D}_{\mathbf{K} \overline{\mathbf{K}}}$ for this experiment is discussed in Appendix $\mathrm{D}$.

$$
\frac{d \mathrm{~N}(\mathrm{t})}{d t}=\mathrm{N}_{K}(0) \mathrm{BR}_{3 \pi}\left\{e^{-t / \tau_{\mathrm{l}}}+2 \mathrm{D}_{\mathrm{KK}}\left|\eta_{+-o}\right| \cos \left(\Delta m t+\varphi_{+-o}\right) e^{-t / 2 \tau_{\mathrm{s}}}\right\}
$$

This clearly shows that the lifetime of the CP symmetry violating term is $\sim \tau_{\mathbf{s}}$. If the target were $\sim 4 \mathrm{~K}_{\mathrm{s}}$ lifetimes upstream of the decay region the interference term would be negligible compared to that of the target $\sim 1 \mathrm{~K}_{\mathrm{S}}$ lifetime upstream of the decay region. By directly comparing data from upstream and downstream targets, the $\mathrm{CP}$ symmetry violating interference term can be isolated and measured. The technique used for doing this is described in Chapter 9. Figure 1.5 shows the proper time distribution, $R(t)$ : the ratio of the time distribution of the downstream 
target to that of the upstream target for $\eta_{+-0}=\eta_{+-}$for our experimental proper time region. The deviation of $R(t)$ from 1 is the signal for $C P$ non-invariance.

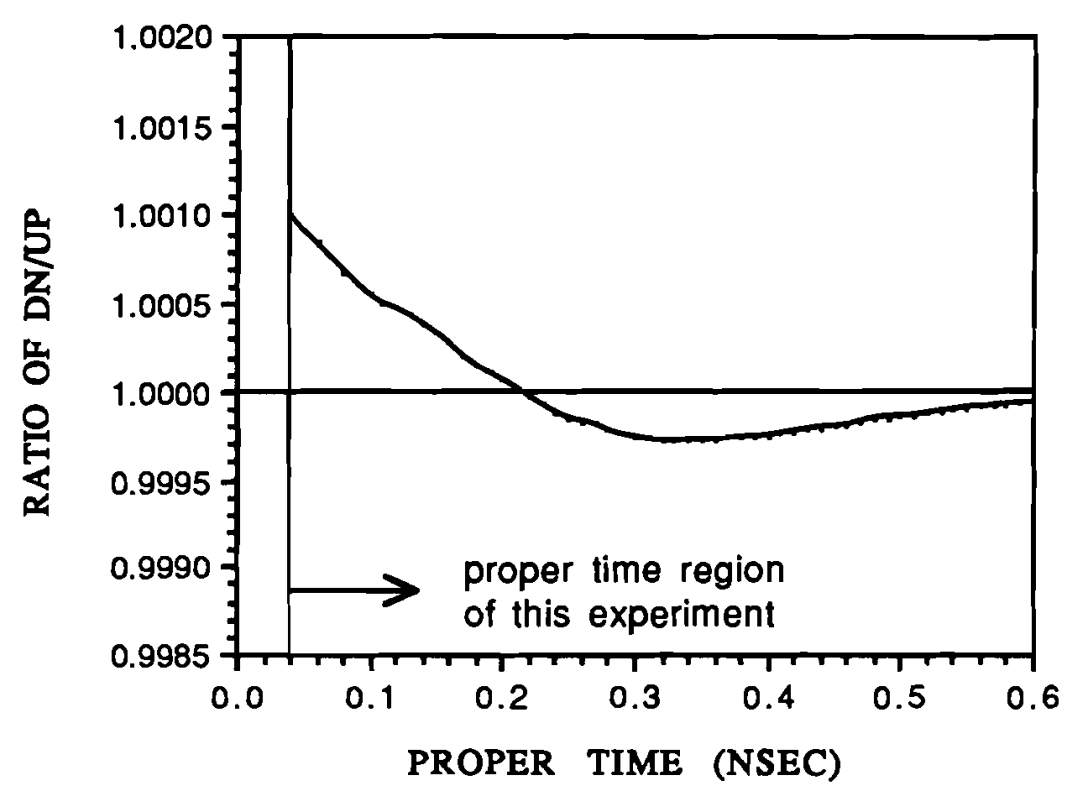

Figure 1.5 Expected Proper Time Ratlo of Events for $\eta_{+-0}=\eta_{+-}$

The Experiment E621 was performed at Fermilab National Accelerator Laboratory (FNAL) in Proton Center in 1985 with a test run in $1984^{(35)}$. 


\section{References for Table 1.1}

(1) J. A. Anderson et. al., Phys. Rev. Lett. 14 (1965) 12,475.

(2) L. Behr et. al., Phys. Lett. 22 (1966) 4,540.

(3) B. R. Webber et. al., Phys. Rev. D 1 (1970) 7,1967.

(4) G. W.Meisner et. al., Phys. Rev. D.3 (1971) 1,59.

(5) Y. Cho et. al., Phys. Rev. D.3 (1971) 7,1557.

(6) F. James et. al., Phys. Lett. 35B (1971) 3,265.

(7) F. James et. al., Nuclear Phys. B 49 (1972) 1.

(8) L. H. Jones et. al., /I Nuovo Cimiento 9 A (1972) 2,151.

(9) M. Metcalf et. al., Phys. Lett. $40 \mathrm{~B}$ (1972) 6,703.

(10) M. Mallary et. al., Phys. Rev. $D 7$ (1973) 7,1953.

(11) M. Baldo-Ceolin et. al., II Nuovo Cimiento 25A (1975) 4,688.

(12) V. V. Barmin et. al., II Nuovo Cimiento 85A (1985) 1,67. 


\section{Chapter Two}

\section{Beamlines}

\subsection{Introduction}

The method used to measure CP violation in the $\mathrm{K}^{\circ} \rightarrow \pi^{+} \pi^{-} \pi^{\circ}$ decay relied on comparing the proper time decay distribution of a mixed $K_{S}+K_{\ell}$ beam to that of a pure $K_{\ell}$ beam by using two side by side proton beams creating two neutral beams ${ }^{(36)}$. This approach had never been tried before in the $\mathrm{K} \rightarrow 3 \pi$ decay measurement of $\eta_{+-0}$. One neutral beam contained a mixture of $K_{s}+K_{\ell}$ mesons while the other beam contained primarily $K_{\ell}$ mesons. Each neutral beam was formed by a collimator imbedded in a large dipole magnet which swept the charged particles in the beam aside.

The Fermilab accelerator provided a beam of $800 \mathrm{GeV}$ protons which was split into three beams for the experimental areas. A Lambertson magnet $500 \mathrm{~m}$ upstream of the first enclosure of our experimental line, PC1, split one of these extracted beams, "the proton beam", into three beams. The central beam of these three proton beams was transported to experiment E621's enclosure PC1. Here an electrostatic septum split the beam vertically. Further downstream two Lambertson magnets seperated the two beams. Quadrupole magnets rotated the two beams to the horizontal plane and focused the beams on the two targets. Since the proton beam direction was roughly north, these two beams were referred to as east and west. Dipole magnets steered the beam towards the targets.

The two targets, upstream and downstream, were moved from one beam to the other approximately every 8 hours in order to reduce systematic effects. This required adjusting the Lambertson and dipole magnets' field strengths in order to hit the targets in their alternate locations. The septum also had to be moved vertically to change the amount of beam on each target.

The resulting neutral beams were seperated horizontally by $4.5 \mathrm{~cm}$ as they entered the decay region of the spectrometer so that one could distinguish the beam 
from which a particular decay originated. The beamline was designed so that both beams passed through a hole in the center of the lead glass array at the downstream end of the spectrometer. This geometry allowed us to distinguish decays from the different beams while still maintaining nearly equal acceptance for decays from either beam.

The requested proton beam intensity for E621 was $5 \times 10^{11}$ protons/spill. The Proton Center beamline region that we controlled consisted of four enclosures called PC1, PC2, PC3 and PC4. In PC1 and PC2 the proton beam was split and steered towards PC3 which enclosed the targets. PC4 contained the spectrometer. The double collimator was embedded in a large magnet between PC3 and PC4. The beamline will be explained in detail in this chapter.

\subsection{Enclosure PC1}

The primary $800 \mathrm{GeV} / \mathrm{C}$ proton beam was extracted from the main ring to enclosure PCO in the proton area. At this point the beam was split into east, center and west for the three proton lines. The Proton Center beam was sent to PC1 where it was steered towards PC2 and split vertically. A schematic of enclosure PC1 is shown in Figure 2.1.

Upon entering PC1 the beam went through a segmented wire ion chamber, here after referred to as a SWIC, which was used to determine the entering beam position. The two quadrupole magnets in PC1 focused the beam on the SWIC at the entrance of enclosure PC2. A secondary emission monitor or SEM measured the number of protons in the beam. This number was recorded on tape for each beam spill. The dipole magnets PC1WD bent the beam mostly west but also down as they were rotated by $12^{\circ}$ from the vertical. As the beam passed through PC1D it bent down, and then was split vertically as it passed through the electrostatic septum which was set at 91 Kilovolts. The SWIC at the exit of PC1 could be used to monitor the beam seperation at this point. 


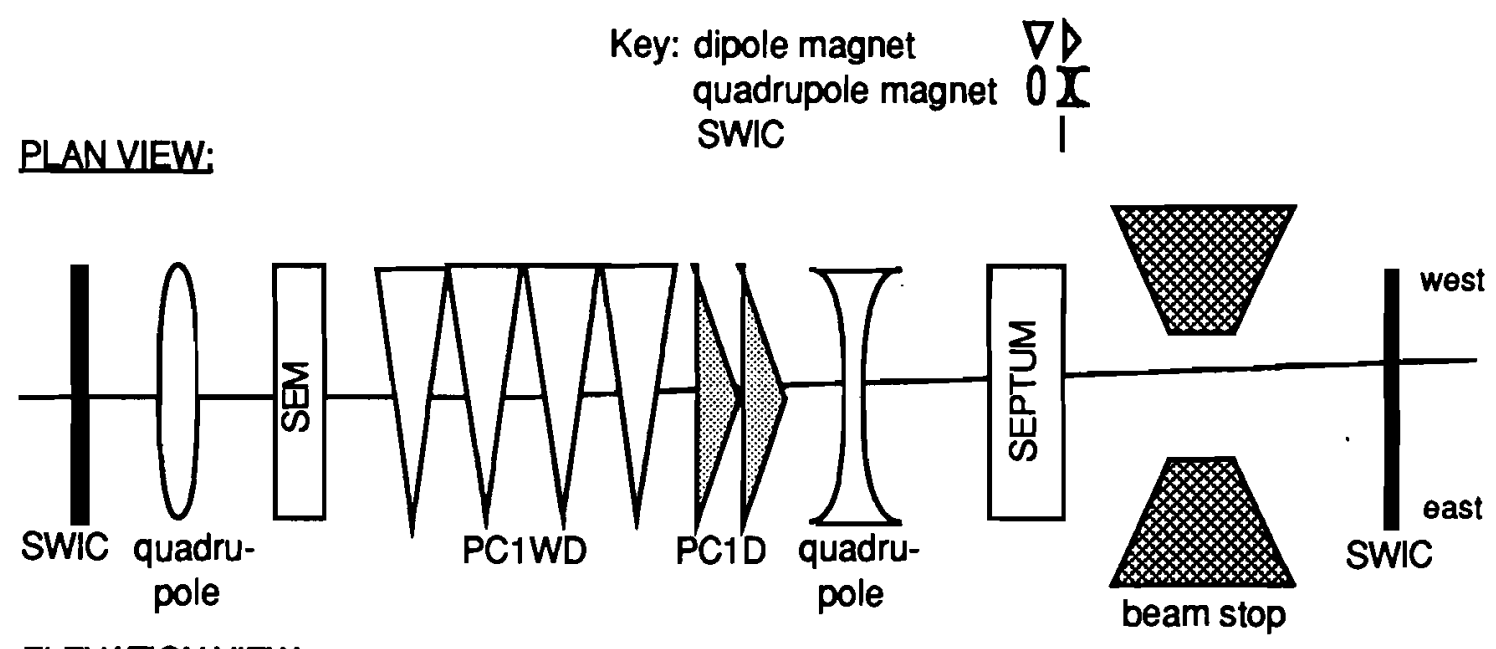

\section{ELEVATION VIEW:}

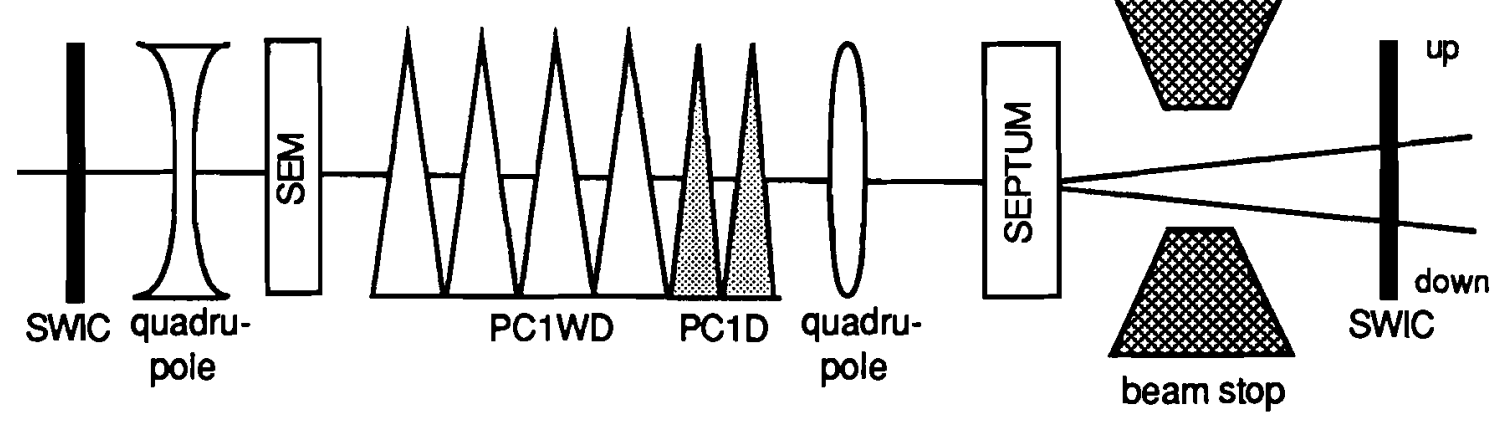

Figure 2.1 Enclosure PC1 ( 2 positions of beamline apparatus are given in Appendix A)

\subsection{Enclosure PC2}

In PC2 the lower beam was rotated up and east in order to obtain two horizontally seperated beams, and quadrupole magnets were used to focus the beam on the targets downstream. This second enclosure, PC2, is shown in Figure 2.2.

PC2 was $527.8 \mathrm{~m}$ downstream of PC1 in order to allow the two beams to seperate more vertically before entering the Lambertson magnets. Once again a SWIC at the entrance to the enclosure displayed the beam size and seperation. The Lambertson magnets had two gaps in them seperated by $0.32 \mathrm{~cm}$. The field free gap was circular and the other gap had a vertical magnetic field. What eventually would become the east beam passed through the lower field free hole while the west beam bent downward in the magnetic field of the upper hole. Once through the Lambertson magnets the beams were well seperated vertically and one needed to be rotated to the horizontal plane. The two sets of quadropole magnets in PC2 accomplished this. They 
both focused the beam spot size at the SWIC in front of the downstream target and rotated the lower, off-axis beam up and to the east. The last two sets of magnets in PC2 were horizontal and vertical trim magnets which positioned the beam on the targets.

\section{PLAN VIEW:}

$$
\begin{array}{ll}
\text { Key: dipole magnet } & \nabla \\
\text { quadrupole magnet } & 0 \text { S } \\
\text { SWIC } & \text { I }
\end{array}
$$

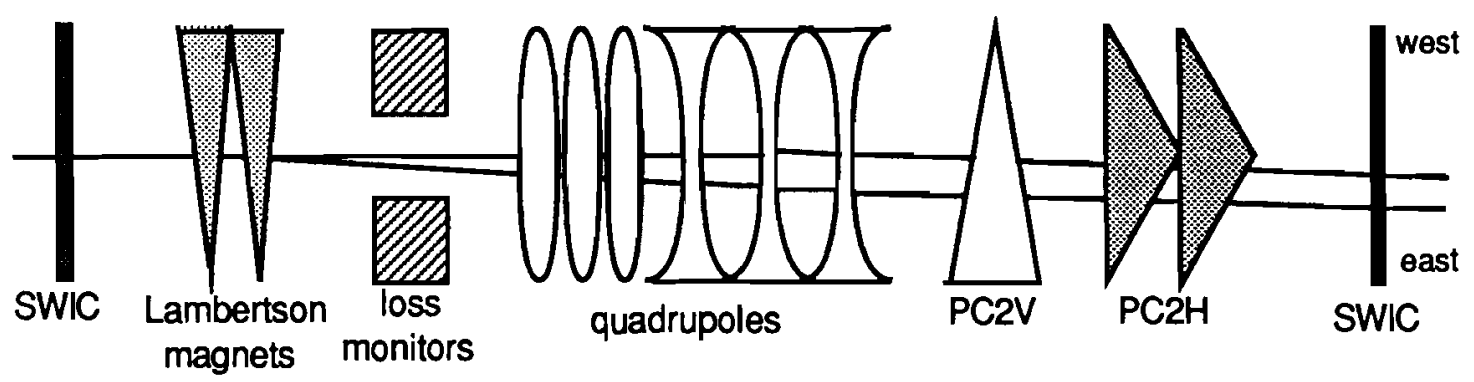

\section{ELEVATIONVIEW:}

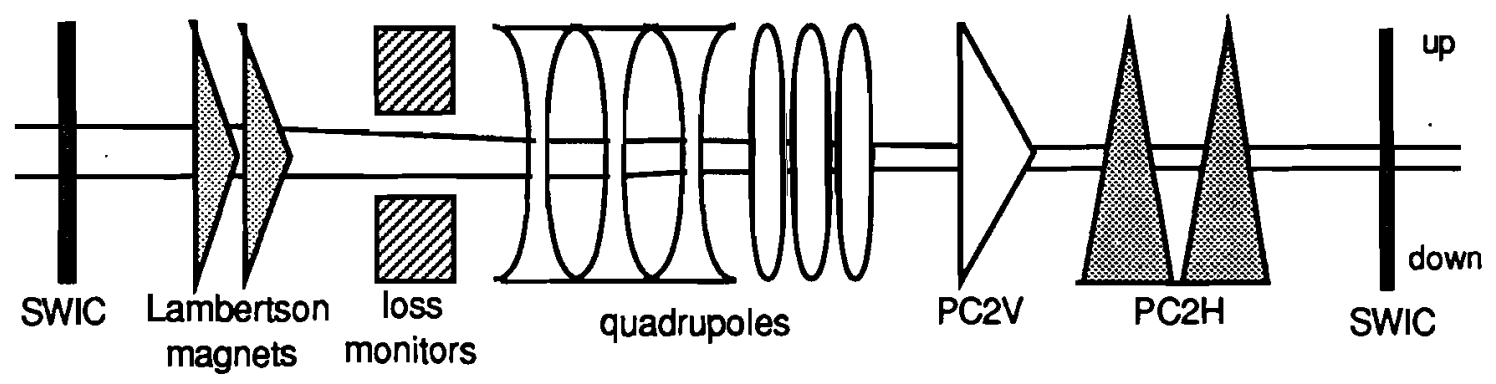

Figure 2.2 Enclosure PC2 (z positions of beamline apparatus are given in Appendix A)

\subsection{Enclosure PC3}

The final enclosure before the spectrometer was PC3, shown in Figure 2.3. It was located $26.8 \mathrm{~m}$ downstream of PC2, and contained both the targets. A large dipole magnet, M1, at the end of the enclosure contained the collimator and swept the charged portion of both beams aside.

Downstream of the SWIC at the entrance to PC3 there were five dipoles, PC3E, which bent both beams east. These dipoles, along with the $\mathrm{PC} 2 \mathrm{H}$ dipoles of enclosure PC2, were used to steer the beam horizontally on to the east target.

Bending the beams to hit both targets using a single set of magnets was a 
delicate procedure. The Lambertsons in enclosure PC2 bent the west beam on to the west target since they had no effect on the east beam. In this way one could adjust the two beams horizontally to hit the two targets by using PC3E and $\mathrm{PC} 2 \mathrm{H}$ to hit the east target and then using PC2W to hit the west target. Vertically only one set of magnets needed to be used since the targets and beams were at nearly the same vertical position. In practice we steered the beam vertically with PC2V on to the downstream target as it was the smaller of the two targets.

The upstream target was rectangular measuring $1.26 \times 1.26 \times 19.09 \mathrm{~cm}^{3}$. The downstream target was cylindrical, $9.58 \mathrm{~cm}$ long with a $0.35 \mathrm{~cm}$ diameter. In order to reduce the high energy photon content of the neutral beams, both targets were made of Hevimet, an alloy of $98 \%$ tungsten and $2 \%$ copper. The SWIC's in front of the targets monitored the beam shape and position on the targets. The downstream target had a SWIC with $1 \mathrm{~mm}$ wire spacings for greater precision in order to steer the beam to hit the smaller target. All other SWIC's had $2 \mathrm{~mm}$ wire spacings.

Key: dipole magnet
SWIC

\section{PLAN VIEW:}

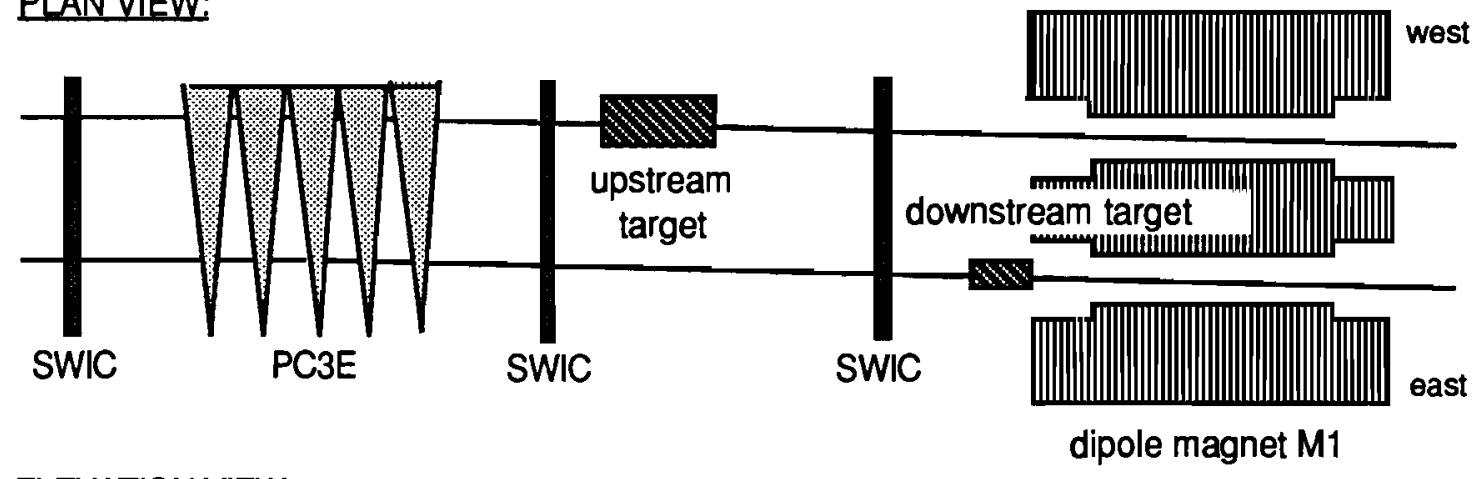

\section{ELEVATION VIEW:}

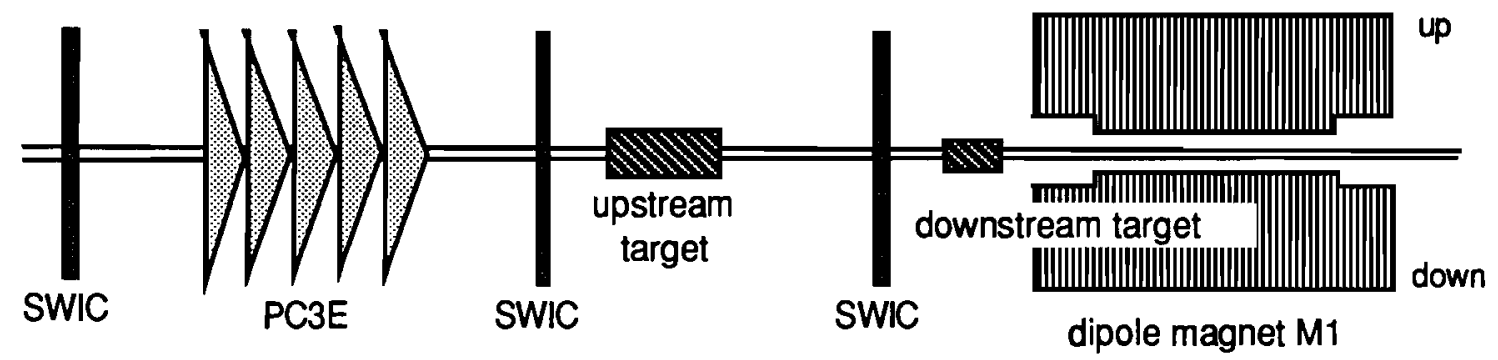

Figure 2.3 Enclosure PC3 ( 2 positions of beamline apparatus are given in Appendix A) 
After passing through all the beamline magnets the beam width at the upstream target was approximately $2 \mathrm{~mm}$ both horizontally and vertically. The beam width at the downstream target, $25 \mathrm{~m}$ downstream, was $2 \mathrm{~mm}$ horizontally and $1.5 \mathrm{~mm}$ vertically. The secondary beams produced by the proton target collisions were mostly pions and photons. These two beams passed through the double collimator which was embedded in a $7.31 \mathrm{~m}$ long dipole magnet (M1) with a magnetic field strength of 3.57 Tesla at a full current of 3600 amp for an integrated B.dl of 26.1 \pm 0.3 Tesla-meters. This magnet was used to sweep all charged particles aside including the residual proton beam. Downstream of magnet $M 1$ the beam was nearly $99 \%$ gammas and neutrons and the remaining $1 \%$ was neutral $\Lambda$ 's, $K^{{ }^{\prime}}$ s, and $\Xi{ }^{\circ}$ 's in decreasing order of abundance.

The two channels in the collimator were inclined towards each other. At the entrance to $M 1$ the two channel centers were $5.08 \mathrm{~cm}$ apart and at the downstream end of M1 they were $4.46 \mathrm{~cm}$ apart. The collimator was designed such that the two beams crossed at the hole in the lead glass array located at the end of the spectrometer. A diagram of the collimator is shown in Figure 2.4. Actual dimensions of the collimator are given in Appendix $A$.

An orthogonal coordinate system for the experiment was defined such that the $z$ axis was parallel to the west neutral beam with the origin at the downstream face of the M1 magnet. The $y$-axis was positive upward making $\mathrm{z}$ north and $\mathrm{x}$ west. Typical target positions in this coordinate system are given in Table 2.1.

\begin{tabular}{|c|c|c|c|c|c|c|}
\hline \multirow[b]{2}{*}{ IARGE } & \multirow[b]{2}{*}{6} & \multicolumn{2}{|c|}{ East Beam } & \multicolumn{2}{|c|}{ West Beam } & \multirow{2}{*}{$\begin{array}{c}\text { X Beam } \\
\text { Sigma }\end{array}$} \\
\hline & & $x$ & $Y$ & $x$ & $Y$ & \\
\hline & 2072 & $-5.31 \mathrm{~cm}$ & $0.43 \mathrm{~cm}$ & $1.65 \mathrm{~cm}$ & $0.17 \mathrm{~cm}$ & $2 \mathrm{~mm}$ \\
\hline am & $-726 \mathrm{~cm}$ & $-4.75 \mathrm{~cm}$ & $0.08 \mathrm{~cm}$ & $0.28 \mathrm{~cm}$ & $0.11 \mathrm{~cm}$ & $1.5 \mathrm{~mm}$ \\
\hline
\end{tabular}

Table 2.1 Target Positlons 
PLAN VIEW

west

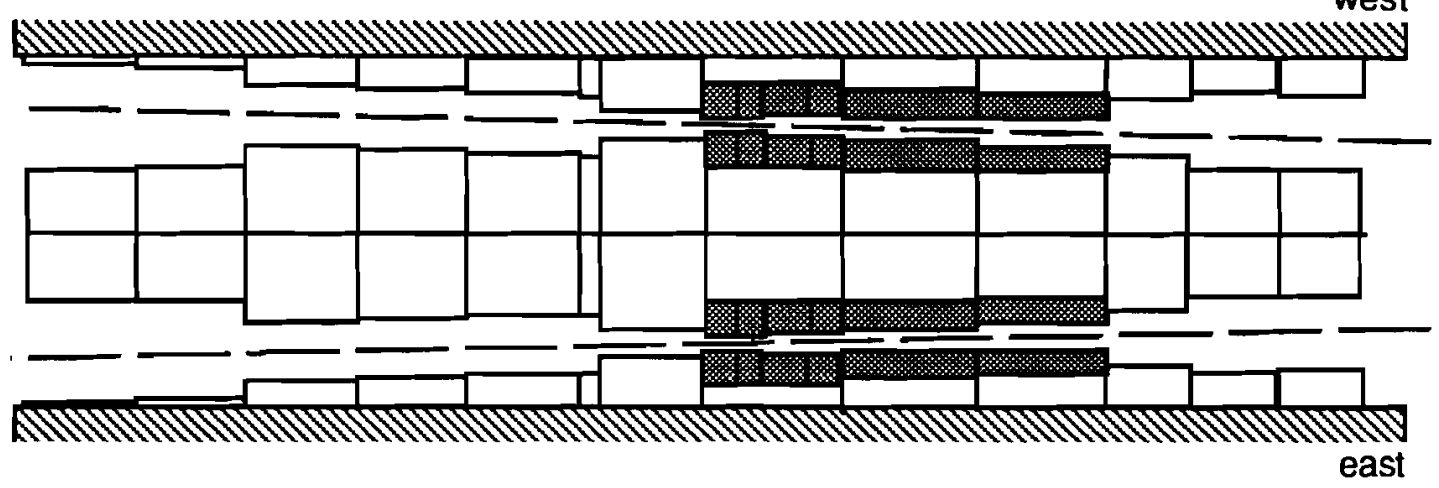

ELEVATION VIEW

up

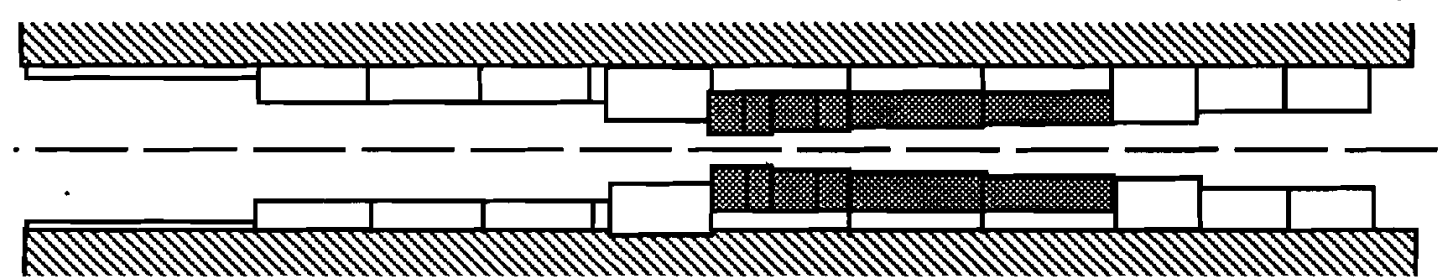

$$
\begin{array}{ll}
\text { (not drawn to scale) } & \begin{array}{l}
\text { Tungsten Inserts } \\
-
\end{array} \\
\square \quad \text { Deutral Beam Center Line } \\
\square \text { Copper Inserts }
\end{array}
$$

Figure 2.4 E621 Collimator (not drawn to scale, dimensions in Appendix A) 


\section{Chapter Three}

\section{The Spectrometer}

\subsection{Introduction}

Enclosure PC4 contained the spectrometer for E621 with the M1 magnet as the wall between PC3 and PC4. Figure 3.1 shows the layout of the experimental apparatus. The decay of interest was $\mathrm{K}^{\circ} \rightarrow \pi^{+} \pi^{-} \pi^{\circ}$ where the $\pi^{\circ}$ immediately decays into two $\gamma$ s. The spectrometer measured the momentum vectors of two charged particles and the position and energy of the two photons. The electronic trigger for the experiment required that a neutral particle enter the decay region and two oppositely charged particles leave it. For the $\mathrm{K}_{\mathbf{3}}$ trigger two neutral showers were required in the lead glass array.

Upon exitng $\mathrm{M} 1$ the beam primarily consisted of neutral particles. The scintillator $V_{1}$ detected any charged particles entering the decay volume. A null signal in $V_{1}$ was required in the trigger to ensure a neutral decay. The scintillation counter V1 marked the start of the decay volume. A second scintillation counter appropriately named DK was located $18.7 \mathrm{~m}$ downstream of $\mathrm{V} 1$. It marked the end of the decay volume and a signal from it was required in the trigger. If a $\mathrm{K}^{0}, \Lambda^{0}, \Xi^{0}$ or any other neutral particle decayed into any number of charged particles in this region, DK would detect them. The signal from DK was also used as a time reference point for the rest of the trigger.

Muons were also produced by the beam interacting with the target and many passed through the hyperon magnet M1. Several sets of toroids surrounded the decay pipe to reduce the muon flux through the spectrometer. The decay pipe was lined with $\mathrm{Mu}$ metal to eliminate any stray magnetic fields produced by the toroids. Powering the toroids did not affect the background rate in the spectrometer so they were not powered during the experiment. The added material of the toriods around the decay pipe did greatly reduced the muon flux through the chambers which had been a problem in the 1984 test run of E621. 


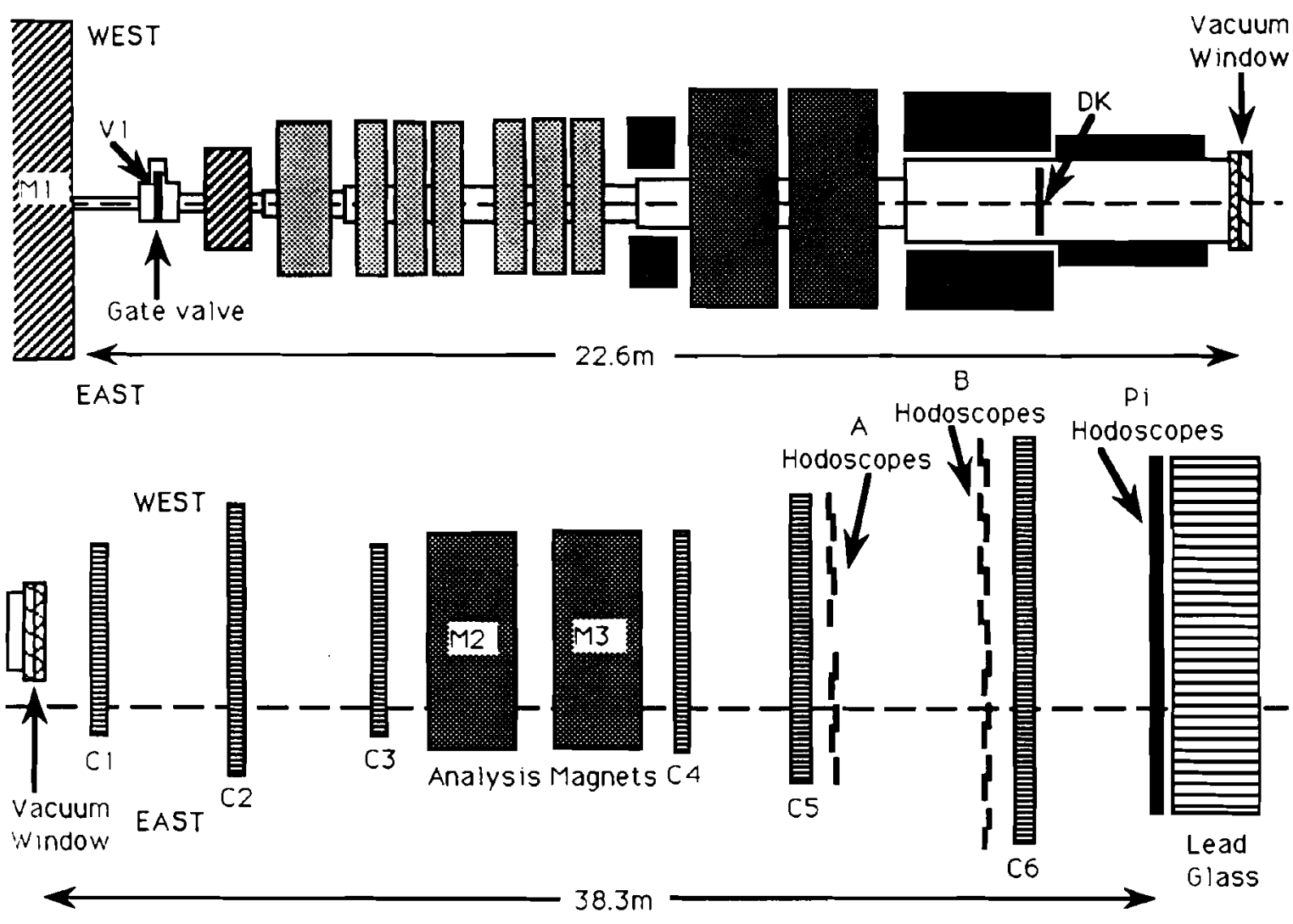

\begin{tabular}{lcc} 
Posotion & ZCenter(meters) & Note: \\
\hline M1 & 0.0 & Scintillator \\
V1 & 0.86 & Spoliers \\
DK & 19.5 & Toroids \\
C1 & Magnets \\
C2 & 23.2 & Shielding Block \\
C3 & 26.9 & Chambers \\
M2 & 30.5 & \\
M3 & 32.3 & \\
C4 & 34.6 & \\
C5 & 36.6 & \\
A Hodoscopes & 41.6 & \\
B Hodoscopes & 42.3 & \\
C6 & 48.4 & \\
Pi Hodoscopes & 60.2 & \\
Lead Glass Array 60.9 &
\end{tabular}

Figure 3.1 The Spectrometer: Plan View (not drawn to scale) 
The remainder of the spectrometer detected charged particles with a vee topology Six multi-wire proportional chambers or MWPC'S and two hodoscope arrays were used to record the positions of the charged particles. The wires of one of the three chambers upstream of the analyzing magnets, C2, was tilted at $45^{\circ}$ in order to resolve any ambiguity in the tracks. The analyzing magnets, M2 and M3, gave charged particles a transverse momentum of $1.553 \mathrm{GeV}$ in order to determine the momentum of the charged particles. The other three chambers and the two arrays of scintillation hodoscopes were downstream of the analyzing magnets. The hodoscopes were positioned between the fourth and fifth chambers. The hodoscopes were used to form a trigger based on the momentum ratio of the two charged tracks and to identify two charged tracks downstream of the analyzing magnets. The trigger will be described in Section 4.2. The MWPC's and the hodoscopes are described in Sections 3.2 and 3.4 respectively.

A lead glass array downstream of the MWPC's was used to measure the energies and positions of the photons from the $\pi^{0}$ decay. An array of scintillation counters, the $\mathrm{Pi}$ hodoscopes, in front of the lead glass identified charged clusters in the lead glass array. A 3 radiation lengths thick sheet of lead directly in front of the glass started the electromagnetic showers in order to minimize the energy loss out the back of the lead glass array. The lead glass array and the Pi hodoscopes are described in Sections 3.3 and 3.5 respectively.

The multiple coulomb scattering in the spectrometer was kept to a minimum by reducing the material in the beamline. The decay region was in a continuous vacuum (approximately $35 \mathrm{mTorr}$ ) with the beamline. The DK and V1 counters were made of $.32 \mathrm{~cm}$ thick scintillator and were in the vacuum. A leak in the vacuum developed in the collimator and was aggravated by changing the polarity of M1. During these times the vacuum increased an order of magnitude to around 300 mTorr for a few hours. The downstream end of the decay pipe was covered with a mylar sailcloth window. Both the vacuum and the mylar window were used to keep multiple coulomb scattering in the spectrometer to a minimum. Helium bags were used between all the MWPC's and inside the analysis magnets M2 and M3. The largest contribution to multiple coulomb scattering was the $A$ and $B$ hodoscope arrays. They were made of $.63 \mathrm{~cm}$ thick scintillator, wrapped in black tape, totalling 0.016 radiation lengths of material. Since the A and B hodoscope elements overlapped, a particle could pass 
through two thicknesses of each array. Most particles would however not pass through the overlap region of either the A or B hodoscope elements. The total number of radiation lengths in the spectrometer was 0.081 if one considered only one thickness of both the $A$ and $B$ hodoscopes. A table of the apparatus and the corresponding radiation lengths of material is shown in Appendix $B$.

\subsection{Multi Wire Proportional Chambers (MWPC's)}

MWPC's consist of parallel anode wires in a plane between two planes of cathode wires. Each anode wire acts as an independent detector. When a particle passes through a chamber it leaves an ionization trail. Several of the electrons from this primary ionization drift towards an anode wire creating their own avalanche of electrons all drifting towards the anode wire. Thus, a signal is detected on the anode wire which is connected to an amplifier and latch. The latch is gated by the experiment trigger. The chamber readout process is described in Section 5.3.

Our MWPC's used $.25 \mathrm{~mm}$ gold-coated tungsten wires with $2 \mathrm{~mm}$ spacing as the anode sense planes. The high voltage cathode planes were $.64 \mathrm{~mm}$ copper-berylium wires with $1 \mathrm{~mm}$ spacing and were kept at a voltage to ensure maximum efficiency for that chamber, typically $\sim-2500$ volts. The planes were seperated by about 1 $\mathrm{cm}$ in $\mathrm{z}$ with alternating high voltage and sense planes, for a total of five planes. The gas mixture used in the MWPC's was $5 \%$ freon mixed with argon bubbled through methylal at $0^{\circ} \mathrm{C}$. Typical MWPC chamber voltages and sizes are listed in Table 3.1.

\begin{tabular}{cccc} 
& INSTRUMENTED & & \\
MWPC & WIRES XXY & APERATURE & VOLTAGE \\
\cline { 3 - 4 }$C 1$ & $256 \times 128$ & $0.51 \times 0.25 \mathrm{~m}^{2}$ & $-2800 \mathrm{~V}$ \\
$\mathrm{C} 2$ & $128 \times 128$ & $0.51 \times 0.51 \mathrm{~m}^{2}$ & $-2790 \mathrm{~V}$ \\
$\mathrm{C} 3$ & $256 \times 128$ & $0.51 \times 0.25 \mathrm{~m}^{2}$ & $-2770 \mathrm{v}$ \\
$\mathrm{C} 4$ & $320 \times 128$ & $0.64 \times 0.25 \mathrm{~m}^{2}$ & $-2950 \mathrm{v}$ \\
$\mathrm{C} 5$ & $640 \times 192$ & $1.28 \times 0.384 \mathrm{~m}^{2}$ & $-3000 \mathrm{v}$ \\
$\mathrm{C} 6$ & $640 \times 192$ & $1.28 \times 0.384 \mathrm{~m}^{2}$ & $-2940 \mathrm{v}$ \\
Table 3.1 & Chamber Aporatures and Voltages &
\end{tabular}

Chamber $\mathrm{C} 2$ was the uv chamber tilted at $45^{\circ}$. The pions from the kaon decay 
are still close together by the time they reach $\mathrm{C} 2$ and thus one does not need a large active chamber region to detect them. On the other hand, the photons from the $\pi^{0}$ in the $\mathrm{K} \rightarrow 3 \pi$ decay are well seperated at chamber $\mathrm{C} 2$ and need a large aperature. Therefore MWPC C2 had only about half the chamber instrumented.

\subsubsection{MWPC Efficiencies}

Relative overall average MWPC chamber efficiencies were determined from the reconstructable vee topology track on a tape by tape basis. While the efficiencies determined in this manner are not necessarily the actual single-track efficiencies, they are proportional to them and provided a check that the chambers were operating properly.

For a given event, if no hit was found in a given chamber to correspond to the reconstructed track, an inefficiency was registered. For each chamber, the efficiency was relatively flat except for sharp dips that corresponded to very inefficient (most likely due to slight mis-timing) amplifier channels. An average overall $x$ and $y$ plane efficiency was determined for each chamber for each tape. Variations in efficiencies occurred due to changes in humidity, high voltage and gas mixture. Typical $x$ and $y$ plane efficiencies are shown in Table 3.2 for the six MWPC's.

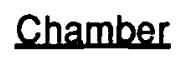

C1

$\mathrm{C} 2$

C3

$\mathrm{C} 4$

C5

C6

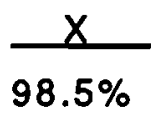

$98.0 \%$

$97.5 \%$

$99.0 \%$

$98.0 \%$

$94.0 \%$

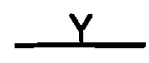

$96.0 \%$

$96.5 \%$

$96.5 \%$

$95.5 \%$

$95.0 \%$

$88.0 \%$

\section{Table 3.2 Chamber Efficiencles}

The efficiencies in Table 3.2 are not the actual single-track efficiencies since two tracks were required in the reconstruction program for the data sample used to determine the efficiencies. This requirement of at least four 'good' hits in the $x$ view, two upstream of the magnets and two downstream, for each charged particle is reflected in the higher efficiency of the x-planes in Table 3.2. For the $y$ view, being 
one straight line through all six chambers, the reconstruction program needed only two hits out of the six chambers to determine a fit for a single charged track versus the four hits out of six required in the $x$ view for a single track. Thus the efficiencies for the $y$-planes are more realistic (and lower) since they are less restricted by the data sample of reconstructable neutral vee decays.

\subsubsection{MWPC Double Hit Probabilities}

When a charged particle passes between 2 wires in a chamber it has a certain likelihood of registering on both wires. This is called the double hit probability and can be measured by counting how often there are two neighboring wire hits associated with a track. Table 3.3 lists the double hit probabilties by chamber and planes. They were found to be constant within errors from tape to tape. The actual double hit probability was calculated matching the reconstructed data hits with a detailed simulation of the experiment. These input and reconstructed double hit percentages for the simulation are listed in Table 3.3. Delta rays, associated with charged tracks which produced signals on neighboring wires, were calculated from the simulation program to be present at the level of $2 \%$ (see Section 7.3 ). The simulation data reconstructed values are included for completeness.

\begin{tabular}{cccc} 
& & $\begin{array}{c}\text { Reconstructed } \\
\text { Chamber }\end{array}$ & $\begin{array}{c}\text { Reconstructed } \\
\text { Real Data }\end{array}$ \\
\cline { 2 - 4 } C1y & $4.2 \%$ & $6.9 \%$ & $7.0 \%$ \\
C1x & $16.5 \%$ & $13.3 \%$ & $13.2 \%$ \\
C2y & $8.0 \%$ & $7.1 \%$ & $7.7 \%$ \\
C2x & $18.0 \%$ & $12.8 \%$ & $13.4 \%$ \\
C3u & $7.8 \%$ & $6.5 \%$ & $7.4 \%$ \\
C3v & $4.5 \%$ & $4.5 \%$ & $5.2 \%$ \\
C4y & $4.7 \%$ & $5.8 \%$ & $6.9 \%$ \\
C4x & $16.6 \%$ & $12.7 \%$ & $13.1 \%$ \\
C5y & $6.8 \%$ & $6.0 \%$ & $6.7 \%$ \\
C5x & $4.3 \%$ & $5.4 \%$ & $4.1 \%$ \\
C6y & $4.5 \%$ & $5.0 \%$ & $6.4 \%$ \\
C6x & $7.6 \%$ & $6.9 \%$ & $7.8 \%$ \\
C. & & &
\end{tabular}

Table 3.3 Double Hit Probability 


\subsection{The Lead Glass Array}

The lead glass array consisted of 86 blocks each $10 \mathrm{~cm} \times 10 \mathrm{~cm} \times 38.4 \mathrm{~cm}$ long or 13.52 radiation lengths stacked as shown in Figure 3.2.

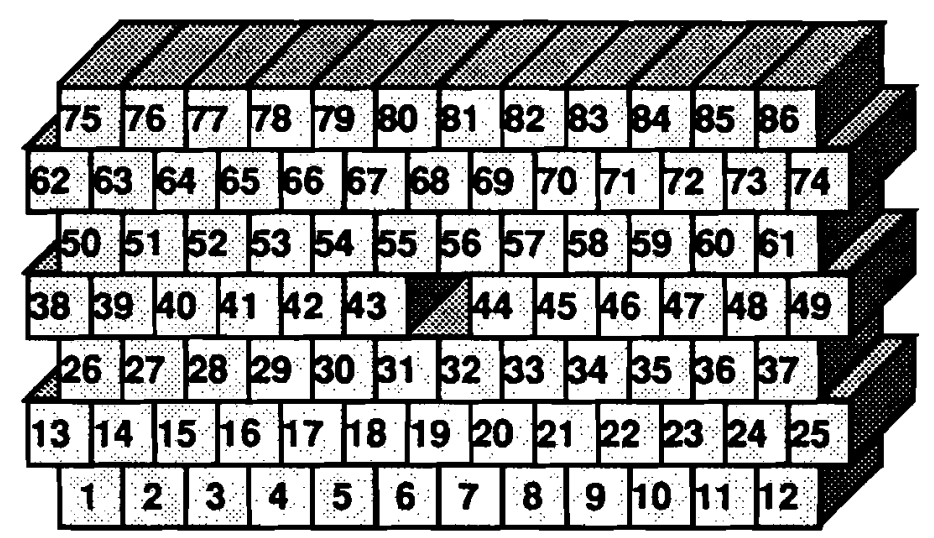

Figure 3.2 The Lead Glass Array

In the test run of E621 it was discovered that there was a significant amount of energy leakage out the back of the glass array(35). This produced a non-linear response. To correct this problem a lead sheet $1.9 \mathrm{~cm}$ thick was placed $2 \mathrm{~cm}$ in front of the lead glass array. Both the lead sheet and the lead glass array had a $10 \mathrm{~cm} \times 10$ $\mathrm{cm}$ hole in the center for the neutral beam. The SF-2 lead glass was $50 \% \mathrm{PbO}, 41 \%$ $\mathrm{SiO}, 5 \% \mathrm{~K}_{2} \mathrm{O}$ and $3 \% \mathrm{Na}_{2} \mathrm{O}$ by weight with a radiation length of $2.84 \mathrm{~cm}$.

Each block was attached to a phototube which measured the light from the Cerenkov radiation from the electromagnetic shower caused by the photon. The entire lead glass array was in an air-conditioned box in order to keep the temperature of the photo tubes constant. All the high voltage supplies for the array were also air conditioned due to their erratic behavior when the humidity and temperature rose.

Each phototube had two outputs. One output went to the control room where it was split into two. One of these two control room signals was input to a Lecroy 2280 Analog to Digital Converter (ADC) which measured the charge from the phototube integrated over $200 \mathrm{~ns}$. The other went to an Emmitter Coupled Logic (ECL) discriminator with two outputs. One of the outputs from the discriminator went to a Lecroy 2770 Time to Digital Converter (TDC) which determined the time of the 


\section{7}

pulses into the Glass Cluster Finding, GCF, trigger processor. The other discriminator output went to the GCF trigger processor itself. The threshold of this ECL discriminator was set relatively high, $25 \mathrm{mV}(\sim 2 \mathrm{GeV})$, since photons of interest deposited large amounts of energy in the glass. The set of glass signals input to the GCF trigger processor did not include the outermost blocks in the array. Section 4.5 explains the details of the GCF trigger processor.

The other signal from the phototubes went to ECL discrimnators in the beamline area and then to the control room on twisted pair cables to another set of TDC's. These TDC's measured all 86 blocks' timings and used a much lower, $10 \mathrm{mV}$, threshold on their discriminator than the GCF trigger processor's TDC's in order to obtain as much time information as possible. Both sets of TDC's timings were used to reject background from accidentals.

\subsubsection{Lead Glass Array Energy Resolution}

The same track fitting program as described in Section 6.5 was used in reconstructing the $e^{+} e^{-}$tracks. The track data yielded the momentum of both particles. From this a correspondence between pulse heights in the glass measured by the ADC's and the energies of the incident particles measured by the spectrometer was determined. Because of the limited lateral extent of an electromagnetic shower, only the signals from the block with the largest ADC signal and its six nearest neighbors were used in determining the shower energy. The pulse heights of the ADC signals, $\mathrm{A}_{j i}$, for the $j^{\text {th }}$ block in the cluster $i$ are known. With $\mathrm{E}_{i}$ as the track energy for cluster $i$, one can determine calibration constants $C_{j}$ for each block $j$ in the lead glass array by minimizing the quantity in equation 3.1 .

$$
\mathrm{F}=\sum_{i}\left(\mathrm{E}_{i}-\sum_{j} \mathrm{C}_{j} \mathrm{~A}_{j i}\right)^{2}
$$

Figure 3.3 shows a typical E/P plot for a lead glass block next to the hole in the lead glass array and one $25 \mathrm{~cm}$ away where $\mathrm{E}$ is the energy of the shower as measured by the lead glass and $P$ is the energy of the electron (positron) as measured by the spectrometer. 


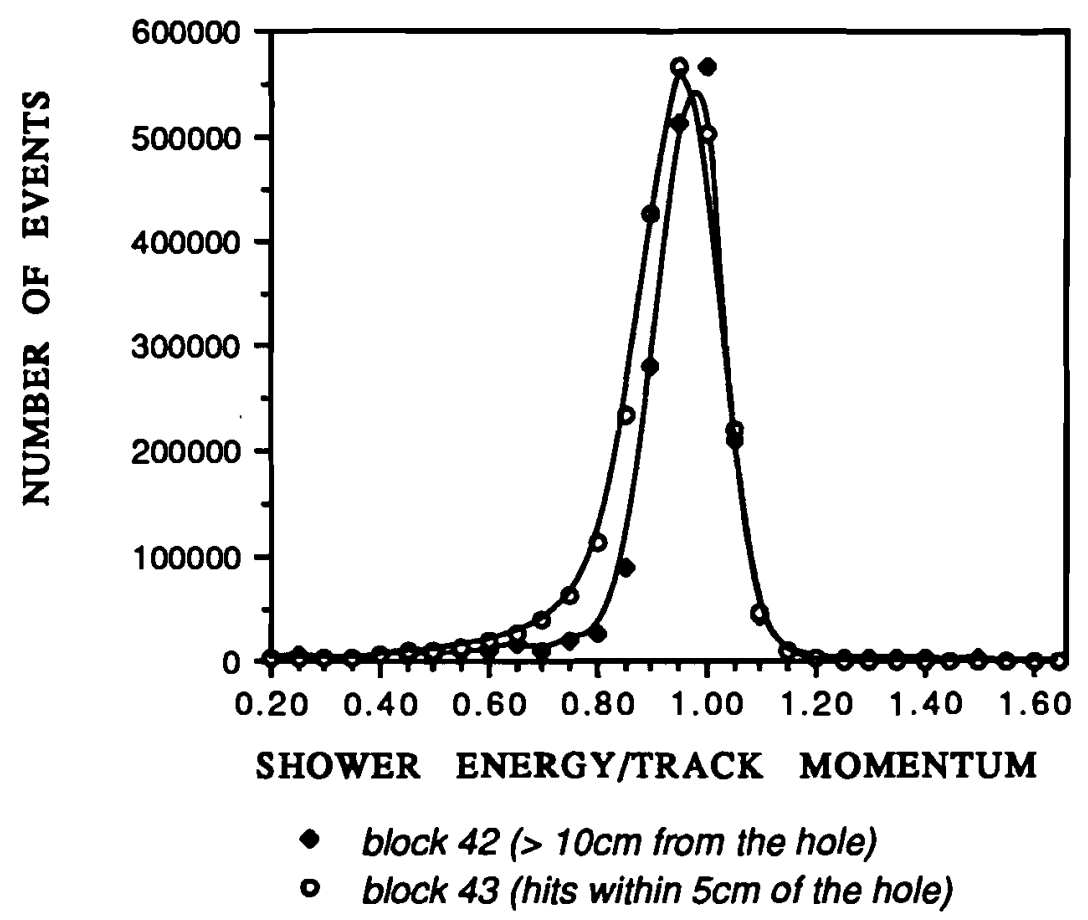

Figure 3.3 E/P Plots

Another set of calibration constants was determined by using photons from the $\pi^{\circ}$ decays in the $\mathrm{K}_{3 \pi}$ data sample. Since there were many of these $\pi^{\circ}$ decays on every tape, the $\pi^{0}$ mass could be used to detect any change in the glass calibration. It was found that the $\pi^{\circ}$ mass decreased with time from $133 \mathrm{MeV}$ to $110 \mathrm{MeV}$ in several weeks. The high rates in the lead glass array due to the residual neutral beam caused radiation damage to the lead glass blocks, especially those near the center of the array, changing their response. By determining tape by tape calibration constants from the $\mathrm{K}_{3 \pi}$ data sample I could correct for the loss of response by increasing the calibration constants. A plot of how the calibration constants changed with time is shown in Figure 6.14.

The energy resolution for the lead glass array can be determined from the halfwidth of the distribution $\mathrm{E}_{\text {glass }}-\mathrm{E}_{\text {track}}$. This was fit to $\sigma(\mathrm{E}) / \mathrm{E}$ to obtain:

$$
\sigma(\mathrm{E}) / \mathrm{E}=0.22 / \sqrt{\mathrm{E}}
$$

where $\mathrm{E}$ is the energy in $\mathrm{GeV}$. This resolution is averaged over the entire array 
for clusters more than half a block $(5.08 \mathrm{~cm})$ from the hole and the outer edge of the array. A more complete discussion of lead glass response is given in section 7.6.

The calibration of the lead glass and a complete discussion of the photon position resolution for this array is given in Section 6.4 and Section 6.5.3.1 respectively.

\subsection{A and B Hodoscopes}

The $A$ and $B$ hodoscopes are shown in Figure 3.4. The signals from the $A$ and $B$ hodoscopes were discriminated and latched. A signal on either side of the hole in each array was required in order for an event to be written to tape. The Memory Look-up Unit (MLU) trigger processor described in Section 4.3, used the $A$ and $B$ hodoscopes to determine if an event had two oppositely charged tracks with symmetric slopes in the $y-z$ plane. The MLU trigger processor allowed only one hit on each side of each hodoscope array. The MLU trigger processor was used in both the $K_{2 \pi}$ and $K_{3 \pi}$ triggers. The hodoscope efficiencies and positions needed to be well determined in order to simulate the hodoscopes and the MLU accurately.

Each scintillator hodoscope element was $5.7 \mathrm{~cm}$ wide and overlapped with its neighboring element by a few millimeters. These overlap regions were used to determine the exact hodoscope positions. The A hodoscopes were $5.05 \mathrm{~m}$ upstream of the $B$ hodoscopes. The neutral beam passed through the hole in the center of the two arrays.

\subsubsection{A and B Hodoscopes Efficiencies and Noise}

Efficiencies of the $A$ and $B$ hodocopes were determined by examining the $A$ and $B$ hodoscope latch information for events which did not require the $A$ and $B$ hodoscopes in order to be recorded. Specifically we used F · C5 trigger events (see Section 4.2 for a description of the F. C5 trigger). These F. C5 triggers were reconstructed to find a vee topology in the charged tracks. An inefficiency was determined for each element by projecting the charged track to the hodoscope array and checking the hodoscope latches to see if the element registered a hit. In determining efficiencies a good hit was defined by a track passing through an element which registers the hit and has no non-adjacent hits in that array's side. Typical inefficiencies, determined in this manner from reconstructed vee track events, are shown for the $A$ and $B$ hodoscopes in Table 3.4. Non-adjacent noise rates accounted for $1-3 \%$ of this inefficiency, the higher value applying to the inner B hodoscope elements. 


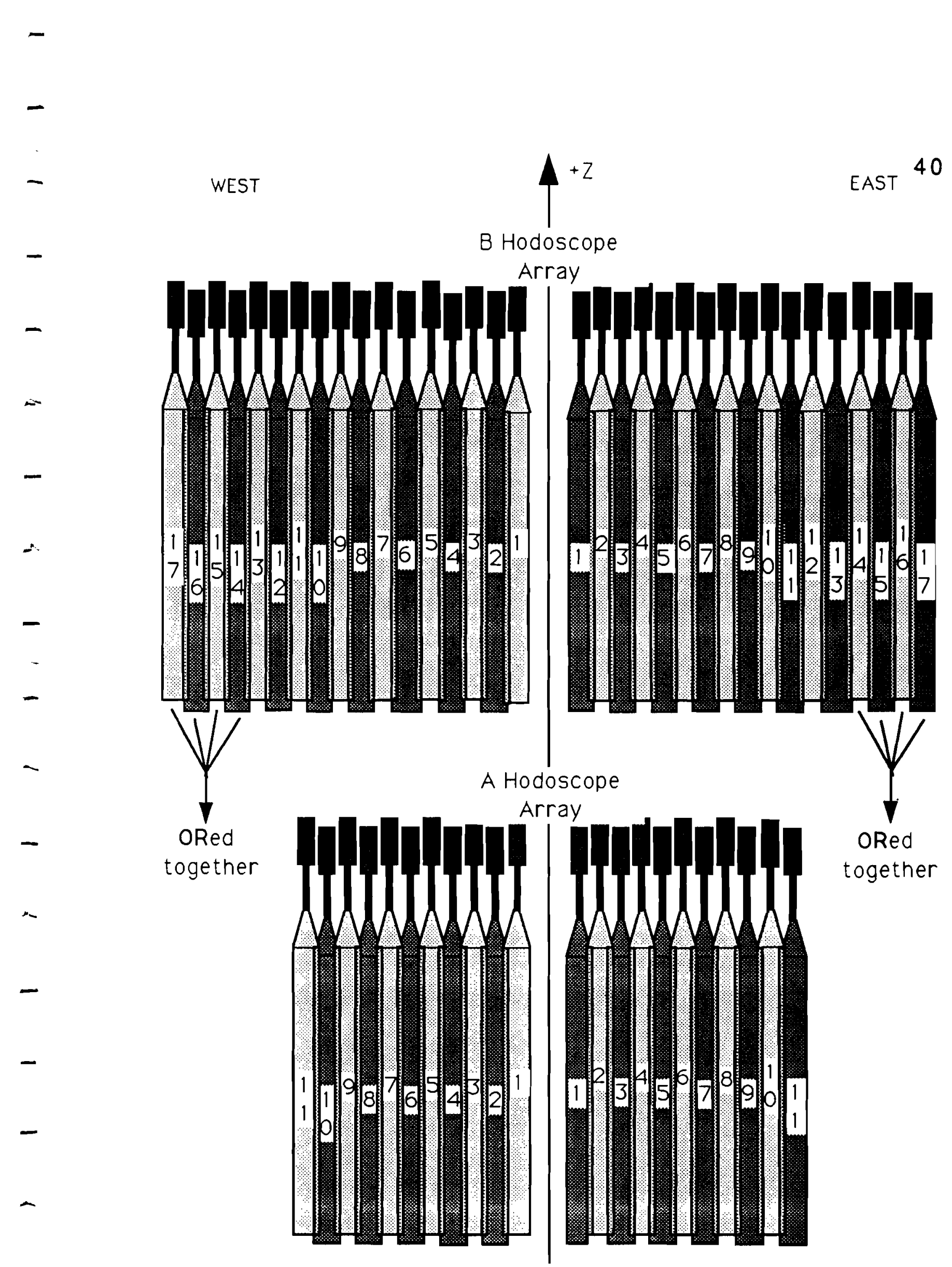

Figure 3.4 A and B Hodoscope Arrays 


\begin{tabular}{lcccc} 
Element & $\begin{array}{c}\text { A West } \\
\text { Inefficiency }\end{array}$ & $\begin{array}{c}\text { A East } \\
\text { Inefficiency }\end{array}$ & $\begin{array}{c}\text { B West } \\
\text { Inefficiency }\end{array}$ & $\begin{array}{c}\text { B East } \\
\text { Inefficiency }\end{array}$ \\
\hline 1 & $5 \%$ & $4 \%$ & $15 \%$ & $16 \%$ \\
2 & $3 \%$ & $5 \%$ & $6 \%$ & $7 \%$ \\
3 & $3 \%$ & $6 \%$ & $7 \%$ & $8 \%$ \\
4 & $5 \%$ & $6 \%$ & $9 \%$ & $9 \%$ \\
5 & $5 \%$ & $7 \%$ & $10 \%$ & $10 \%$ \\
6 & $5 \%$ & $5 \%$ & $6 \%$ & $8 \%$ \\
7 & $6 \%$ & $17 \%$ & $7 \%$ & $7 \%$ \\
8 & $5 \%$ & $9 \%$ & $6 \%$ & $7 \%$ \\
9 & $4 \%$ & $7 \%$ & $5 \%$ & $10 \%$ \\
10 & $8 \%$ & $9 \%$ & $7 \%$ & $8 \%$ \\
11 & $25 \%$ & $18 \%$ & $8 \%$ & $10 \%$ \\
12 & & & $10 \%$ & $10 \%$ \\
13 & & & $8 \%$ & $10 \%$ \\
14 & & & $14 \%$ & $11 \%$
\end{tabular}

Table 3.4 A and B hodoscope Inefficlencles Determined from F•C5 Triggers

\subsection{Pi Hodoscopes}

The Pi hodoscopes were located $76.8 \mathrm{~cm}$ in front of the lead glass array and were used to identify clusters in the lead glass caused by charged particles. Figure 3.5 shows the arrangement of the Pi hodoscopes and how they overlapped the lead glass array. There were five vertical and three horizontal scintillator elements to either side of the hole in the lead glass array. This formed fifteen regions of overlap on each side.

When a charged track passed through one of these fifteen regions it would register in the two overlapping hodoscope elements. These signals were sent to the Glass Cluster Finding (GCF) trigger processor (described in section 4.5). The GCF trigger processor would then identify any clusters it found behind this region of overlap as charged clusters and not use them in its final determination of the number of neutral clusters in the glass array. 


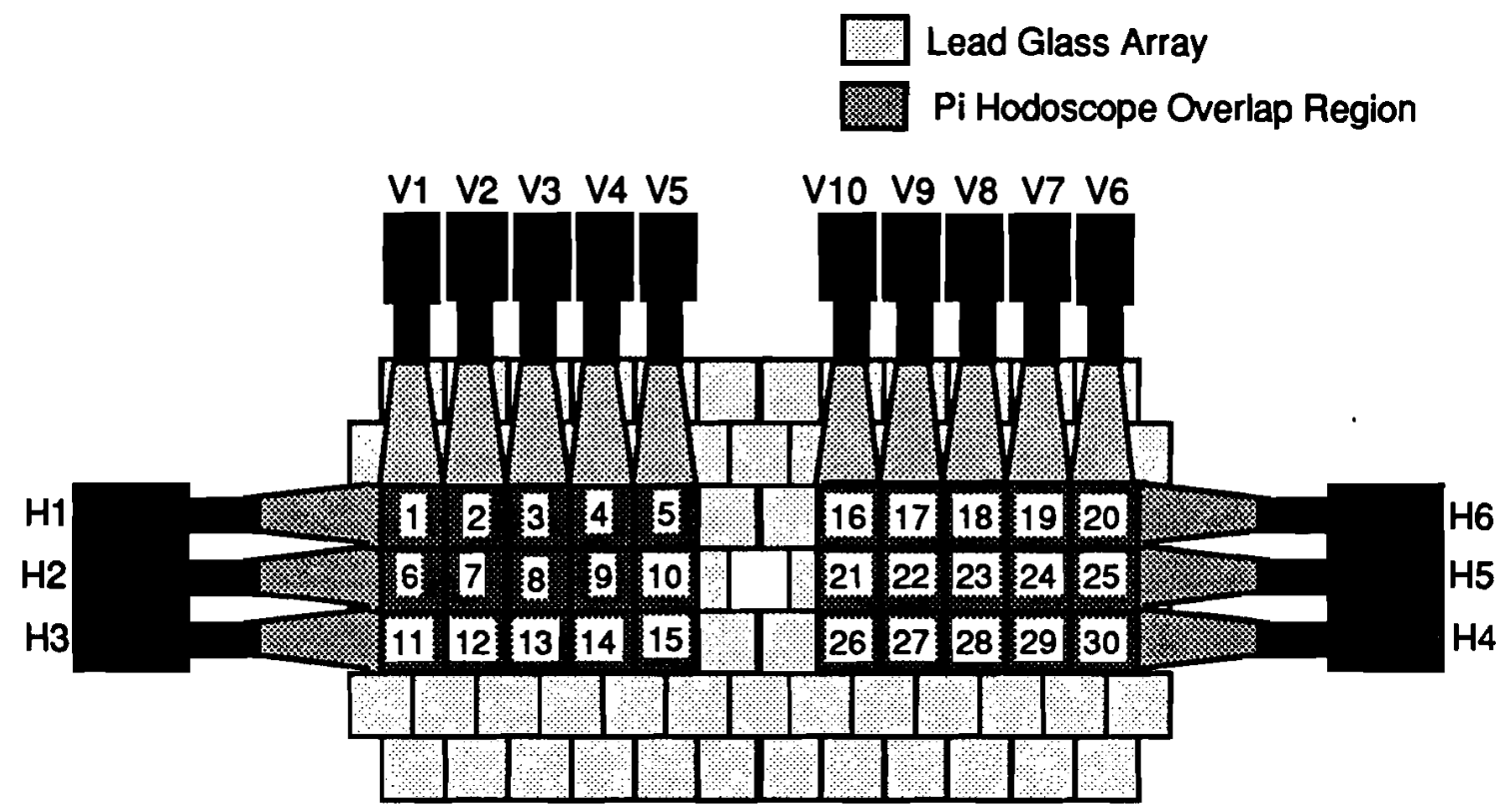

Figure 3.5 PI Hodosccopes LookIng Downstream

Since the Pi hodoscopes were used as vetos for clusters in the glass caused by charged tracks, their inefficiency only accepted more events and thus was not a problem. Typically they were about $89 \%$ efficient.

\subsubsection{Pi Hodoscope Noise}

Noise in the Pi hodoscopes was a more complicated and important effect. While inefficiencies in the array did not lose any events, noise in the array could identify a neutral cluster as charged and thus not add it in to the final determination of the number of neutral clusters in the glass. The GCF trigger processor needed to find at least two neutral showers in order to accept an event in the $K_{3 \pi}$ trigger. Thus, if a neutral glass cluster was accidentally identified as a charged cluster due to noise in the $\mathrm{Pi}$ hodoscopes, the $\mathrm{K}_{3 \pi}$ trigger would not be satisfied and the event would be lost.

There were two possible sources of signals in the Pi hodoscopes not associated with a charged hit. One of these, random noise, was a small effect (less than $0.5 \%$ ) and evenly distributed throughout the array. The other source of Pi hodoscope signals was the photons themselves. When a photon hit the lead glass array behind the hodoscopes there was a chance of charged particle albedo from the electromagnetic shower entering the Pi hodoscopes causing misidentification of a neutral cluster. Another source of noise occurred when a charged pion hit the 
hodoscope array and caused neighborring scintillator elements to register a signal probably due to albedo from the hadronic shower in the lead glass. By examining $\mathrm{K}_{2 \pi}$ triggers, it was determined that this occurred $25-30 \%$ of the time. Of these three effects only the photon self-vetoing due to charged particle albedo was important.

The charged pions were generally well seperated from the photons by the time they reached the lead glass array. A charged pion caused a mis-identification of a neutral shower in the $\mathrm{K} 3 \pi$ data sample only $1 \%$ of the time. This made the hadronic albedo that occurred $25-30 \%$ of the time when a hadron hit the $\mathrm{Pi}$ hodoscopes negligible. This albedo effect was however included in the simulation of the experiment. (see Section 7.6.1)

Pure random noise in the $\mathrm{Pi}$ hodoscopes was determined for each overlap region from data where no pions hit the Pi hodoscope array and was found to be $\sim 0.5 \%$.

Photon Self-Vetoing:

The photon associated albedo was measured as a photon acceptance efficiency using $\mathrm{K}_{3 \pi}$ candidates from triggers which required one photon cluster in the lead glass, K. G1 triggers (see Section 4.2). I considered events from this trigger that passed all the $\mathrm{K}_{3 \pi}$ cuts to ensure that the lead glass portion of the data corresponded to a $\pi^{\circ}$.

Efficiencies were determined for the 15 regions shown in Figure 3.6. The photon efficiency was defined as the ratio of events where both photons had no $\mathrm{Pi}$ hodoscope hit in the overlap region over their central hit block to all events.

The dark shaded region corresponds to the portion covered by the Pi hodoscopes where one expects albedo to occur. The efficiencies are shown for each region. Since I used K - G1 triggers, at least one neutral shower is required for the event to trigger. This causes the photon efficiencies to be higher than they actually are. I corrected this by taking the product of the efficiency in the region where the one photon from the K - G1 trigger hit and the weighted average efficiency of the rest of the array. This gave a "photon efficiency" for the K - G1 trigger and thus told how many events we did not see by requiring a $G 1$ in the trigger. The results are shown in Figure 3.6. The symmetry one sees here was valid to within one standard deviation. The only exception is for the center hole region where the efficiency was measured 3.5 sigma below 1.0 despite the fact that no scintillator covered the region. All other regions of less than $100 \%$ efficiency were covered by the $\mathrm{Pi}$ hodoscopes. 
The low efficiency in the center region is most likely due to the fact that the blocks on either side of the hole are half covered with scintilator and, with the large uncertainty in the position of photons in the region due to the hole in the array, there is a smearing of the efficiency lowering the cental area efficiency which should be $100 \%$. This position smearing probably also accounts for the increase in the neighboring efficiency to $92 \%$ versus $72 \%$. The simulation program of the experiment used the values in Figure 3.6.

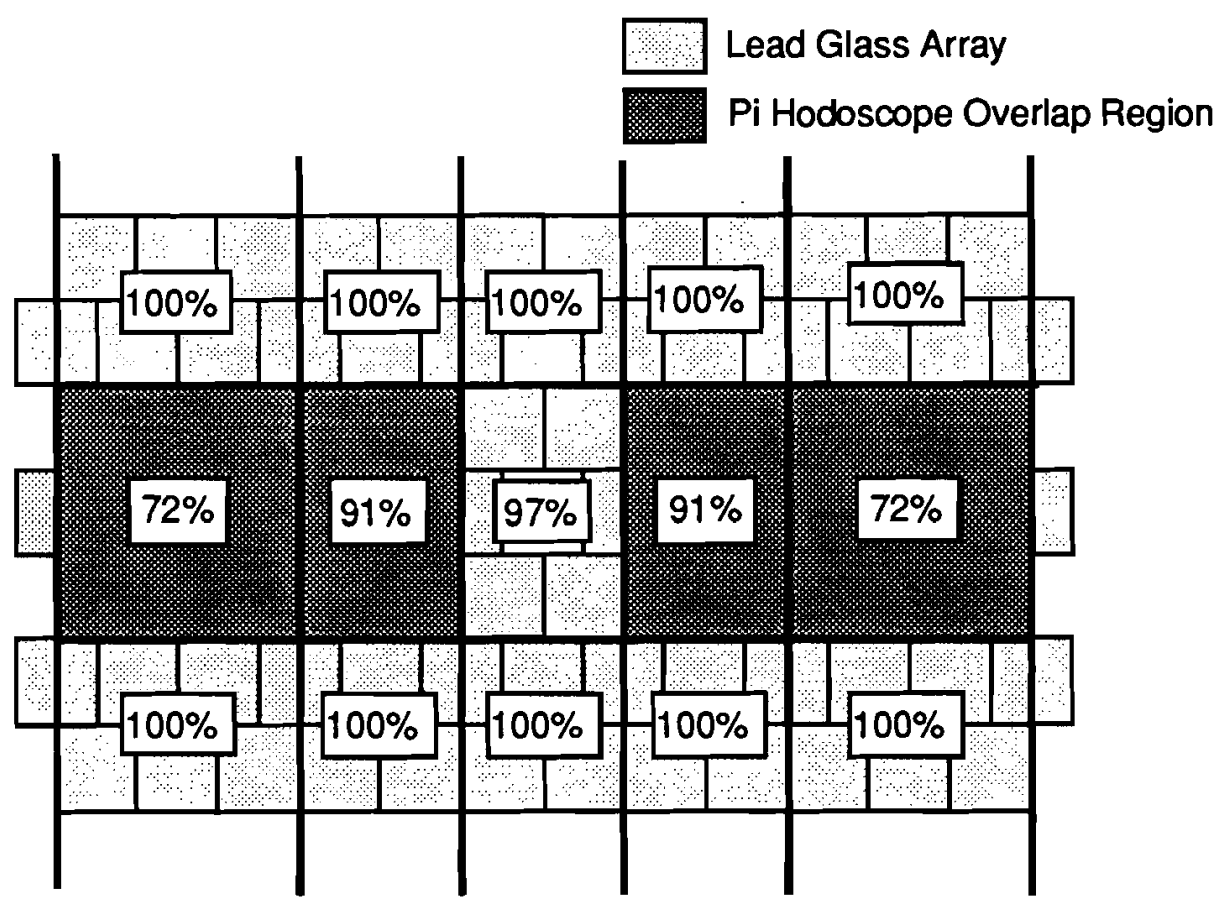

Figure 3.6 Photon Non-veto Llkellhoods 


\section{Chapter Four}

\section{Triggers and Trigger Processors}

\subsection{Introduction}

The design of the $\mathrm{K}_{2 \pi}$ and $\mathrm{K}_{3 \pi}$ triggers was influenced by how they were used. The $\mathrm{K}_{2 \pi}$ trigger was restricted by a prescaler to make up only $\sim 20 \%$ of all triggers written on to tape. The $\mathrm{K}_{3 \pi}$ trigger made up $60 \%$ of all triggers written on to tape. The performance of the chambers limited the proton beam intensity that could efficiently be used and the data acquisition system limited the total number of triggers which could be written to tape during a spill. Due to these limitations, the $\mathrm{K}_{2 \pi}$ and $\mathrm{K}_{3 \pi}$ triggers had to have as high a yield of reconstructable decays as possible. The $\mathrm{K}_{2 \pi}$ was a subset of the $\mathrm{K}_{3 \pi}$ trigger so that any biases created in trying to achieve good yields could be studied at both levels.

A basic "vee" topology trigger helped ensure reconstructable neutral decays, while the MLU and CMR trigger processors used charged particle kinematics to help give a high ratio of kaon to lambda decays. The GCF trigger processor, used in the $\mathrm{K}_{3 \pi}$ trigger, increased the $\mathrm{K} \rightarrow 3 \pi$ to $\mathrm{K} \rightarrow 2 \pi$ ratio by identifying two neutral showers in the lead glass array. Each of the three trigger processors had to process an event in $\leqq 100$ nsec so as not to adversely effect the trigger and data acquisition rate.

The neutral beam produced by the targets contained mainly photons, neutrons, lambdas and kaons in decreasing order of profusion. The photons and neutrons usually went through the apparatus without interacting since there were only 0.081 radiation lengths of material in the experiment. The lambdas, on the other hand, could decay to a proton and a pion and make a charged vee pattern with a topology similar to the charged particles in $\mathrm{K} \rightarrow 2 \pi$ and $\mathrm{K} \rightarrow 3 \pi$ decays. The kaons decaying to $\pi^{+} \pi^{-}$provided a background in the $\mathrm{K}_{3 \pi}$ trigger if a photon requirement were not included in the trigger. The $\mathrm{K}_{3 \pi}$ trigger used three trigger processors to eliminate $\Lambda \rightarrow p \pi$ and $K \rightarrow 2 \pi$ events at the trigger level. The $K_{2 \pi}$ trigger used one of these 
three trigger processors to remove $\Lambda \rightarrow p \pi$ events from its event sample.

In the $\Lambda \rightarrow p \pi$ decay, the proton always has a much greater momentum than the pion. Kaons, on the other hand, have a relatively symmetric distribution of $\pi^{+}$to $\pi^{-}$ momentum. Figure 4.1 shows the ratio of the positive track momentum to the negative track momentum determined from a Monte Carlo simulation for $\Lambda, K \rightarrow 2 \pi$ and $\mathrm{K} \rightarrow 3 \pi$ decays. Selecting a momentum ratio less than 3.0 removes $100 \%$ of the $\Lambda \rightarrow p \pi$ decays, only $1 \%$ of the $K \rightarrow 3 \pi$ and $25 \%$ of the $K \rightarrow 2 \pi$ decays. This is the basis of the MLU and CMR trigger processors.

The momentum ratio was determined from the slope of the charged tracks downstream of the analysis magnets. This was valid because the analysis magnets' momentum transfer of $1.6 \mathrm{GeV} / \mathrm{c}$ was much larger than the transverse momentum of the charged decay products. Thus the charged particles' angles after the magnets were inversely proportional to their momenta and one could compare charged momenta by comparing the slopes of the tracks. The MLU trigger processor and the CMR trigger processor both measured the ratio of the slope of the particle tracks downstream of the analysis magnets. This ratio was required to be between 0.3 and 3.0 in order to identify an event as a kaon.

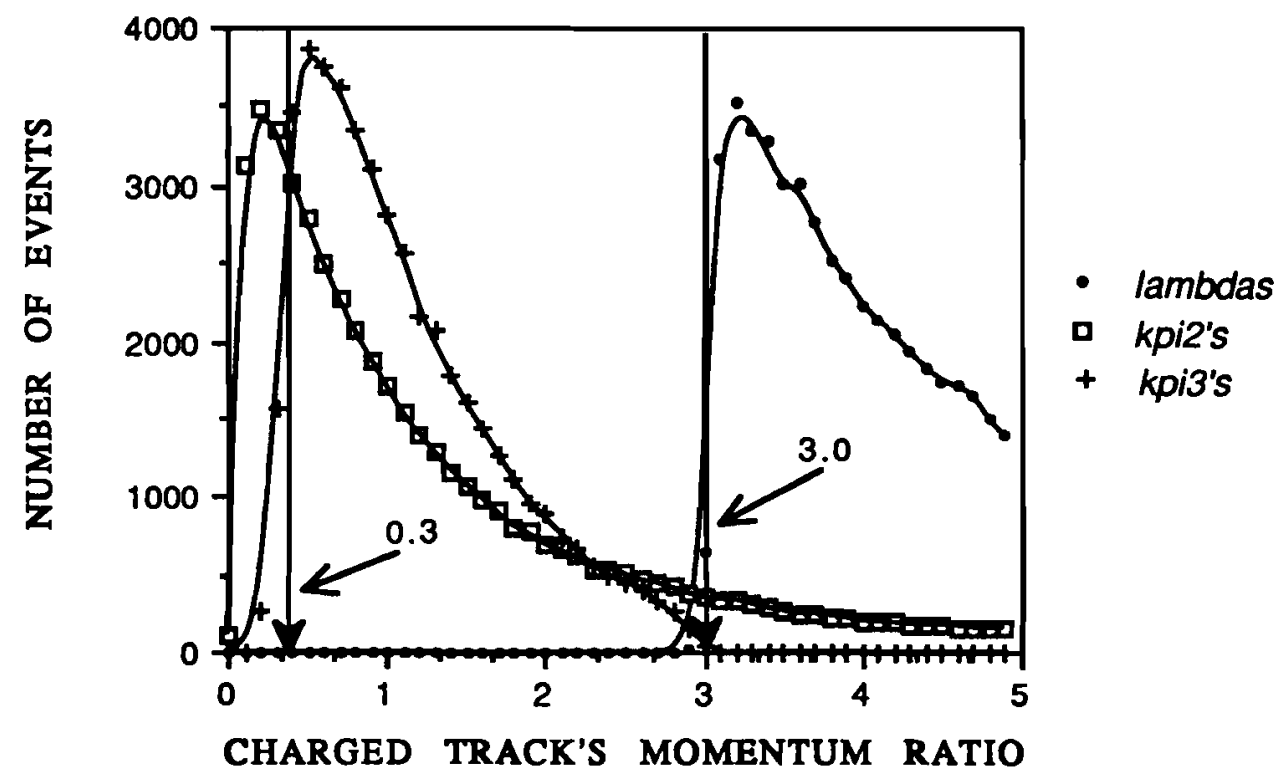

Figure 4.1 Charge Track Mometum Ratlos for Lambdas and Kaons 
The MLU trigger processor calculated a crude charged particle momentum ratio using the $A$ and $B$ hodoscopes. It also vetoed events with more than one hit in each half of the two hodoscope banks. The CMR trigger processor determined a more precise slope (momentum) ratio using chamber hits. Together the two trigger processors removed $95 \%$ of the lambda decays from a simple vee topology trigger while losing $62 \%$ of the $\mathrm{K}^{0} \rightarrow \pi^{+} \pi^{-}$decays and $55 \%$ of the $\mathrm{K}^{0} \rightarrow \pi^{+} \pi^{-} \pi^{0}$ decays. Inefficiencies within the trigger processors themselves only accounted for $10 \%$ of these losses. Most of the $\mathrm{K} \rightarrow 2 \pi$ and $\mathrm{K} \rightarrow 3 \pi$ losses in the trigger processors above that determined from Figure 4.1 were due to inefficiencies in the hodoscopes and chambers on which they relied. This aspect of the individual efficiencies of the MLU and CMR trigger processors is discussed in Sections 4.3.4 and 4.4.4 respectively.

The third trigger processor, the Glass Cluster Finding (GCF) trigger processor, was used to reduce the number of $\mathrm{K}^{0} \rightarrow \pi^{+} \pi^{-}$decays in the $\mathrm{K}_{3 \pi}$ trigger by recognizing and counting clusters of energy in the lead glass array which were not vetoed by a signal in the $\mathrm{Pi}$ hodoscopes in front of the lead glass array. Showers in the lead glass with a hit in the Pi hodoscope overlap region in front of them were assumed to be hadronic showers and thus ignored. The $\mathrm{K}_{3 \pi}$ trigger required the GCF trigger processor to find at least two neutral showers in the lead glass array. This requirement removed $85 \%$ of the $\mathrm{K}^{\circ} \rightarrow \pi^{+} \pi^{-}$candidates and $25 \%$ of the $\mathrm{K}^{\circ} \rightarrow \pi^{+} \pi^{-} \pi^{\circ}$ candidates from a simple vee topology trigger.

\subsection{Basic Electronic Triggers}

Most of the apparatus efficiencies were determined using data from less restrictive triggers, described below, recorded isochronously with the $K_{3 \pi}$ triggers during the course of the experiment. These triggers were subsets of the $K_{3 \pi}$ trigger and thus were used for determining apparatus efficiencies. Figure 4.2 shows the detector and the tracks of a typical neutral particle decay to two charged particles. The full E621 trigger schematic is shown in Figure 4.3.

The ' $F$ ' or "Front" trigger was the electronic AND of $\overline{\mathrm{V} 1}$ and DK. A null signal in V1 identified a neutral particle entering and a signal in DK identified a charged particle leaving the decay region. The $F \cdot C 5$ electronic trigger added the requirement of a signal in chamber 5 . This trigger, $F \cdot C 5$, was used to determine $A$ and $B$ 


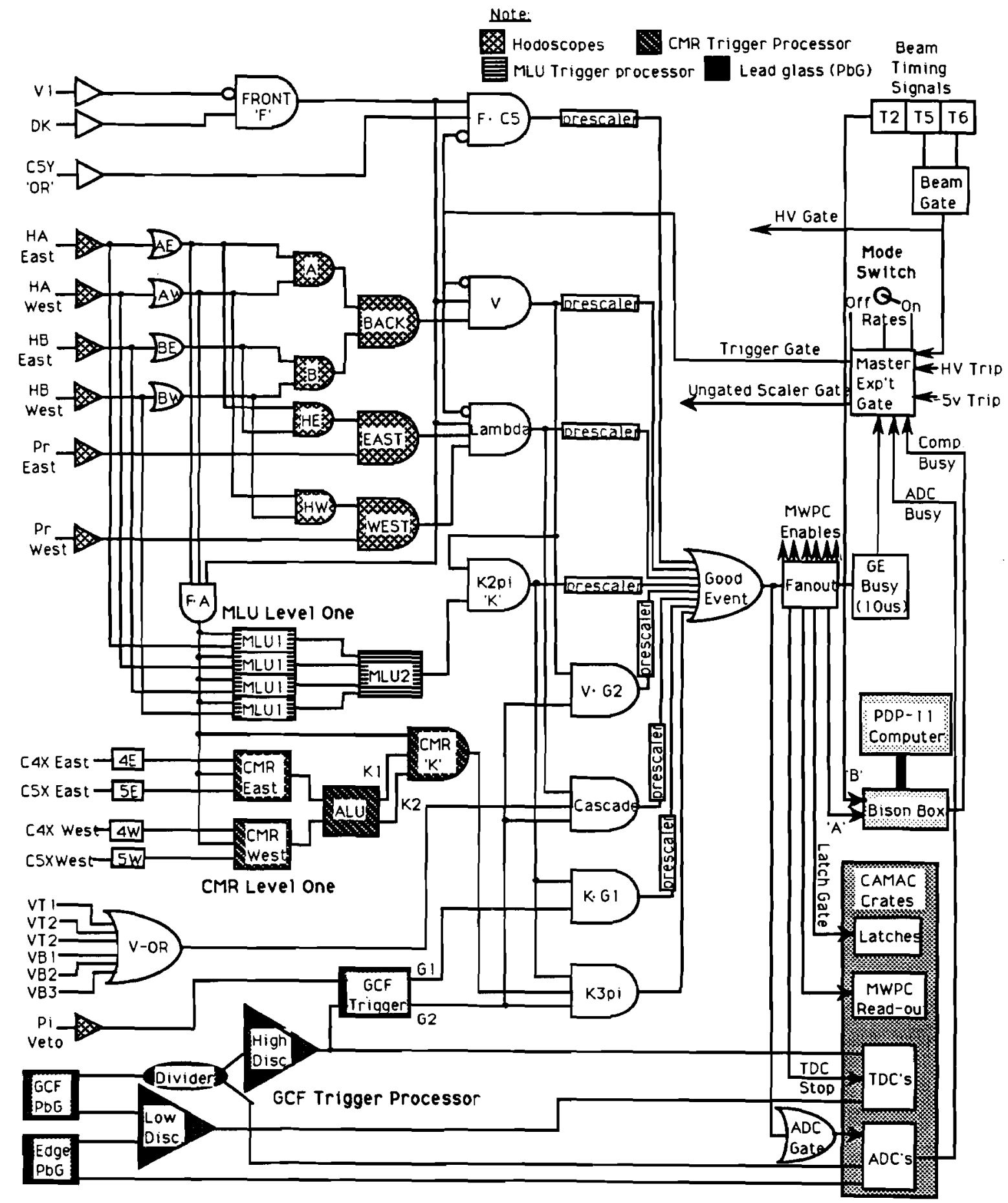

hodoscope efficiencies. The ' $\mathrm{V}$ ' trigger required: $F \cdot\left[\left(H A_{W} \cdot H A_{E}\right) \cdot\left(H B_{W} \cdot H B_{E}\right)\right]=$ ' $V$ ', where $H A_{W}, H A_{E}, H B_{W}$ and $H B_{E}$ are electronic OR's of all the $A(B)$ hodoscope elements on the west (east) side of the beam center line. This extra requirement of a 
hit in each side of each bank of the A and B hodoscopes helped identify two oppositely charged tracks downstream of the analysis magnets M2 and M3. Events passing the ' $V$ ' trigger were used in determining the efficiencies of the MLU and CMR trigger processors.

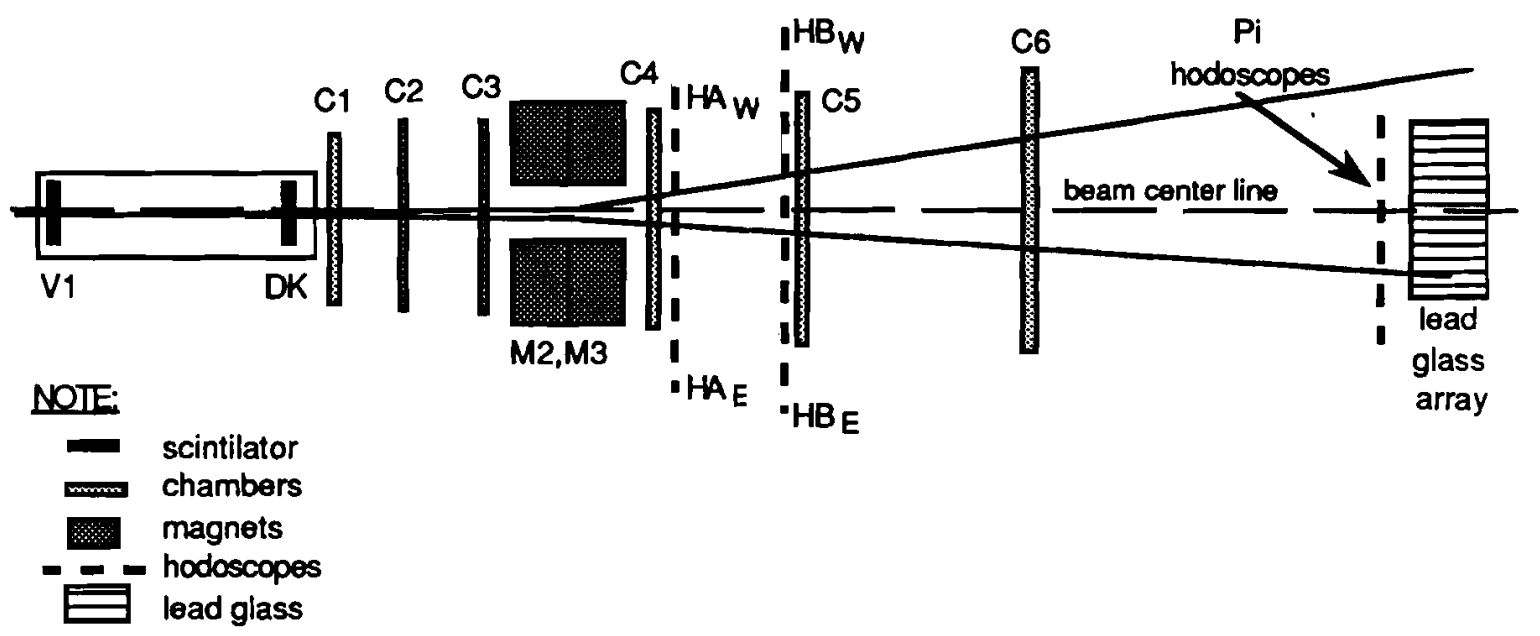

Figure 4.2 Tracks of a Typlcal Partlcle Decay

Another electronic trigger, $K$ (mostly $K \rightarrow 2 \pi$ decays), was used also to determine noise rates in the $\mathrm{Pi}$ hodoscopes and the experiment normalization of the number of kaons produced from the targets. It required the ' $V$ ' trigger to be satisfied and the Memory Look-up Unit (MLU) trigger processor to identify the decay as a possible Kaon decay. The MLU trigger processor used the $A$ and $B$ hodoscopes to measure the slopes of the track candidates downstream of $M 2$ and $M 3$ and required them to be relatively symmetric about $z$. In both the kaon decays to two pions and to three pions, the charged pions are relatively symmetric about $z$ downstream of the analysis magnets since their momenta are approximately equal.

The main trigger samples of interest for the measurement of $\eta_{+-0}$ were the $\mathrm{K}_{2 \pi}$ and the $\mathrm{K}_{3 \pi}$. They both used the " $\mathrm{V}$ " trigger as a foundation while adding trigger proceessor logic to increase the yield of reconstructable $K \rightarrow 2 \pi$ and $K \rightarrow 3 \pi$ decays. Events with slope ratios between 0.3 and 3.0 were identified as kaon decays and all others as lambda decays. The $K_{2 \pi}$ trigger used the $M L U$ trigger processor. The $K_{2 \pi}$ ( $\mathrm{K}$ ) trigger logic was: 


$$
\overline{V 1} \cdot D K \cdot\left(\left(H A_{E} \cdot H A_{W}\right) \cdot\left(H B_{E} \cdot H B_{W}\right)\right) \cdot M L U={ }^{\prime} K '
$$

where MLU requires the MLU trigger processor to identify the event as a kaon.

The $\mathrm{K}_{3 \pi}$ trigger used the MLU and the CMR trigger processors. It also required at least two neutral clusters (or G2) to be found in the lead glass array by the GCF trigger processor in order to eliminate $\mathrm{K} \rightarrow 2 \pi$ decays from the $\mathrm{K} \rightarrow 3 \pi$ trigger. The full $\mathrm{K}_{3 \pi}$ trigger was:

$$
\overline{V 1} \cdot D K \cdot\left(\left(H A_{E} \cdot H A_{W}\right) \cdot\left(H B_{E} \cdot H B_{W}\right)\right) \cdot M L U \cdot C M R \cdot G 2=K_{3 \pi} \cdot
$$

The MLU, CMR and GCF trigger processors are discussed in depth in Sections 4.3-4.5. The special triggers that were prescaled and recorded along with $K_{3 \pi}$ triggers are listed in Table 4.1 along with their typical prescaler factor. A prescaler factor of $2^{9}$ means that 1 of every $2^{9}$ events that satisfy that trigger type will be recorded on tape.

\begin{tabular}{lc} 
IRIGGEB & PRESCALER \\
\hline $\mathrm{F} \cdot \mathrm{C} 5$ & $2^{10}$ \\
$\mathrm{~V}$ & $2^{13}$ \\
$\mathrm{~V} \cdot \mathrm{G} 2$ & $2^{8}$ \\
$\mathrm{~K}=\mathrm{K} 2 \pi$ & $2^{8}$ \\
$\mathrm{~K} \cdot \mathrm{G} 1$ & $2^{8}$ \\
$\Lambda^{0}$ & $2^{13}$ \\
$\Xi^{\circ}$ & $2^{6}$ \\
$\mathrm{~K}_{3 \pi}=\mathrm{K} \cdot \mathrm{CMR} \cdot \mathrm{G} 2$
\end{tabular}

$$
\begin{aligned}
& \text { LOGICDESCRIPTION } \\
& F=\overline{V 1} \cdot D K, C 5=\text { any signal in chamber } C 5 \\
& V=F \cdot B \text { and } B=\left(\left(H A_{L} \cdot H A_{R}\right) \cdot\left(H B_{L} \cdot H B_{R}\right)\right) \\
& G 2=\text { at least two neutral clusters in the GCF } \\
& K=F \cdot V \cdot M L U \text { and } M L U \text { satisfies the } M L U \\
& G 1=a t \text { least one neutral cluster in the GCF } \\
& \Lambda=\overline{V 1} \cdot D K \cdot B \cdot(P 1+P 2) \\
& \Xi=\Lambda \cdot G 2^{(37)} \\
& \text { un-prescaled } K_{3 \pi} \text { trigger, CMR satisfies } K \text { in } \\
& \text { the CMR }
\end{aligned}
$$

\section{Table 4.1 Trlggers and Prescaler Values}

\footnotetext{
- P1 and P2 were scintilator elements to either side of the hole in the lead glass array which were to register the proton from the $\Lambda^{\circ}$ decay
} 
All these trigger results were latched and recorded on tape for each event. The $\mathrm{A}, \mathrm{B}$ and $\mathrm{Pi}$ hodoscope signals were also latched and written to tape. The first and second level MLU trigger processor and CMR trigger processor decisions were latched and written to tape along with the final GCF trigger processor decision and all the lead glass array energy and timing information.

\subsection{Memory Look Up Unit (MLU) Trigger Processor}

\subsubsection{Introduction}

The MLU trigger processor measured the ratio of charged particle slopes by using the hit information from the $A$ and $B$ hodoscope arrays. The MLU trigger processor used Lecroy 2372 Memory Look-up Units, MLU CAMAC programable ECL memory storage modules with $64 \mathrm{~K}$ bits of memory, to determine valid slope ratios.

The MLU trigger processed an event in about $130 \mathrm{nsec}$. It was enabled by :

$$
\bar{V} 1 \cdot D K \cdot\left(H A_{w} \cdot H A_{E}\right) \text {, }
$$

corresponding to a neutral particle decaying with its products causing at least one hit in each side of the $A$ hodoscope array.

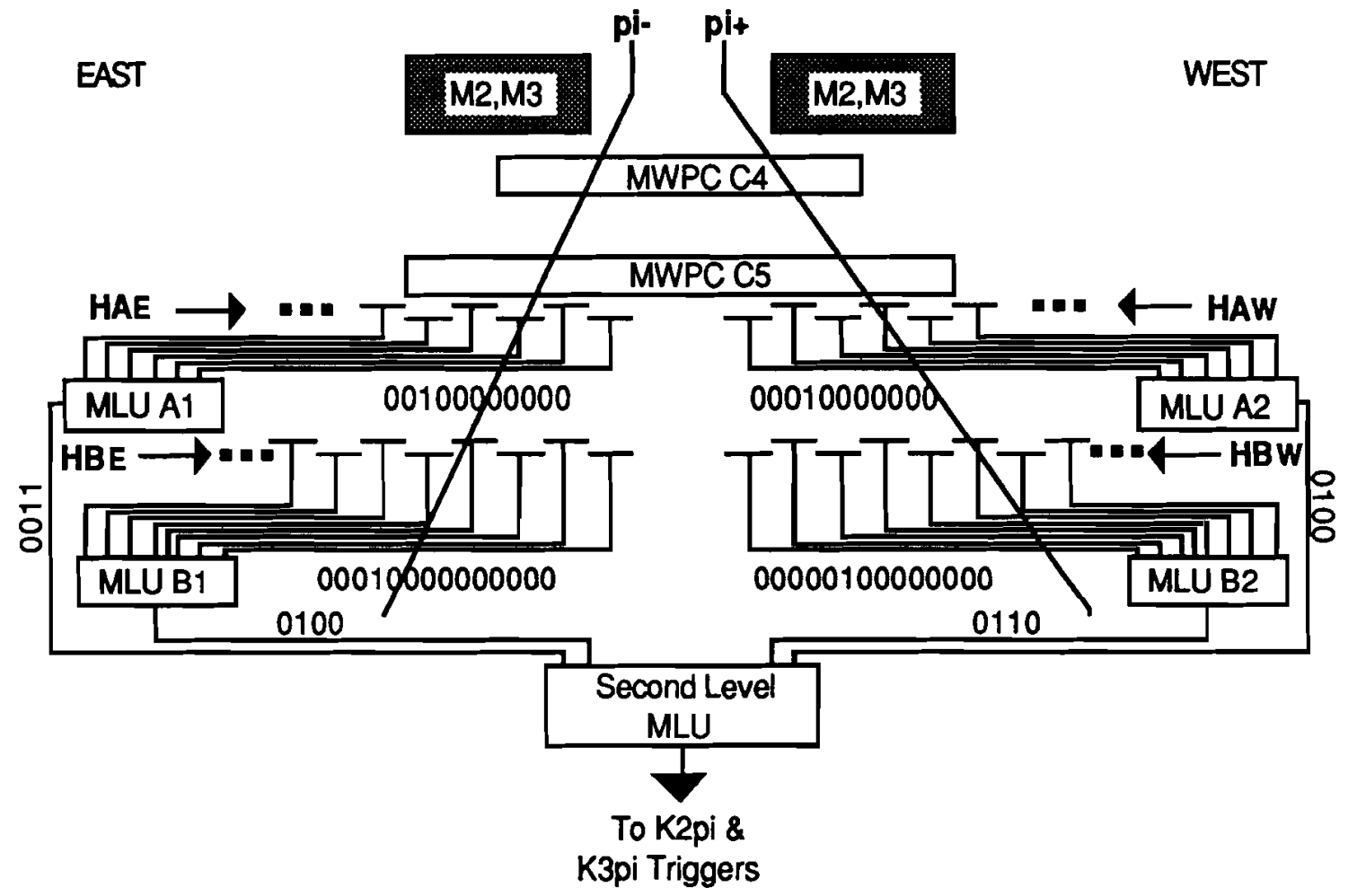

Flgure 4.4 MLU Layout 
Figure 4.4 shows a schematic of the MLU trigger processor layout. The MLU trigger processor had two levels of processing. The first level used the latched $A$ and $B$ hodoscope information to encode the hodoscope hit information. The $A$ and $B$ hodoscope arrays were shown in Figure 3.4. The second level processing of the MLU trigger processor determined if the slope of the tracks corresponding to the first level addresses was between 3.0 and 0.3 . All possible slope ratios for the various combinations of $A$ and $B$ hodoscope hits were loaded into the MLU with a 0 or 1 output pattern depending upon whether the four hodoscope hits corresponded to a slope ratio between 3.0 and 0.3 or not.

\subsubsection{Design : First Level MLU Trigger}

The first level of the MLU trigger consisted of four MLU's which determined the four addresses of the hits in the $A$ and $B$ hodoscopes. The signals from each of the eleven elements on each side in the A hodoscope array went to an ECL discrimator and then to two MLU's. The B hodoscope signals were treated in the same way except for the four outermost elements on each side which were OR'ed together. Thus only 14 signals from the B hodoscopes sent to the remaining two first level MLU's. Each of the four MLU's in the first level then output the 4 bit address of the counter that was hit. If two adjacent counters were hit, the MLU output the inner counter address for the $A$ hodoscopes and the outer counter address for the $B$ hodoscopes. If there were 2 non-adjacent hit counters or no hit counters on a given side the first level MLUs output 1111 or 0000 respectively and the event would be vetoed.

\subsubsection{Design : Second Level MLU Trigger}

The second level MLU trigger consisted of one MLU whose input was the four 4 bit addresses from the four first level MLU's. These 4 addresses defined the counters hit on each side of the center line and thus two slopes. This momentum ratio, for all possible sets of $A$ and $B$ counter hits, was calculated beforehand and assigned a 1 if the ratio was between 3 and $1 / 3$ and $a 0$ if it was not. The second level MLU also was programmed to throw out events whose slopes did not project back to the analysis magnet's gap. A final output of 1 was required by the MLU trigger the $K^{0} \rightarrow 2 \pi$ and $\mathrm{K}^{\circ} \rightarrow 3 \pi$ triggers.

\subsubsection{MLU Trigger Efficiencies}

The MLU had relied on hodoscope elements that were $5.08 \mathrm{~cm}$ wide in order to determine particle slopes and thus lost events due to poor resolution. With $5.08 \mathrm{~cm}$ 
wide counters cutting at a slope of exactly 3 was also impossible. I used a vee trigger Monte Carlo requiring a hit in each side of the $A$ and $B$ hodoscopes to determine the losses due to this. Based on this Monte Carlo, the MLU trigger processor rejected $80 \%$ of lambda decays while losing $10 \%$ of $K \rightarrow 3 \pi$ decays and $32 \%$ of $K \rightarrow 2 \pi$ decays. Multiple hits in the hodoscopes also lost $8 \%$ of both types of kaon decays. Overall the MLU trigger processor rejected $70 \%$ of the lambdas from the beam while losing $46 \%$ and $30 \%$ of the possible $K \rightarrow 2 \pi$ and $K \rightarrow 3 \pi$ events respectively

$V$ trigger data were used to calculate the efficiency of the MLU trigger processor. Overall the first level of the MLU trigger processor was $94 \%$ efficient. Since both the MLU simulation program and the MLU trigger used the latched hodoscope information to determine an answer, this efficiency does not reflect the $A$ and $B$ hodoscope inefficiencies. A discrepancy in the $B$ hodoscope comparison plot turned out to be an inefficiency in the first level of the MLU trigger processor in the B hodoscope east elements 11 and 12 . These channels were generally $38 \%$ and $68 \%$ efficient respectively. The dip corresponding to this MLU inefficiency is pointed out in Figure 4.5.

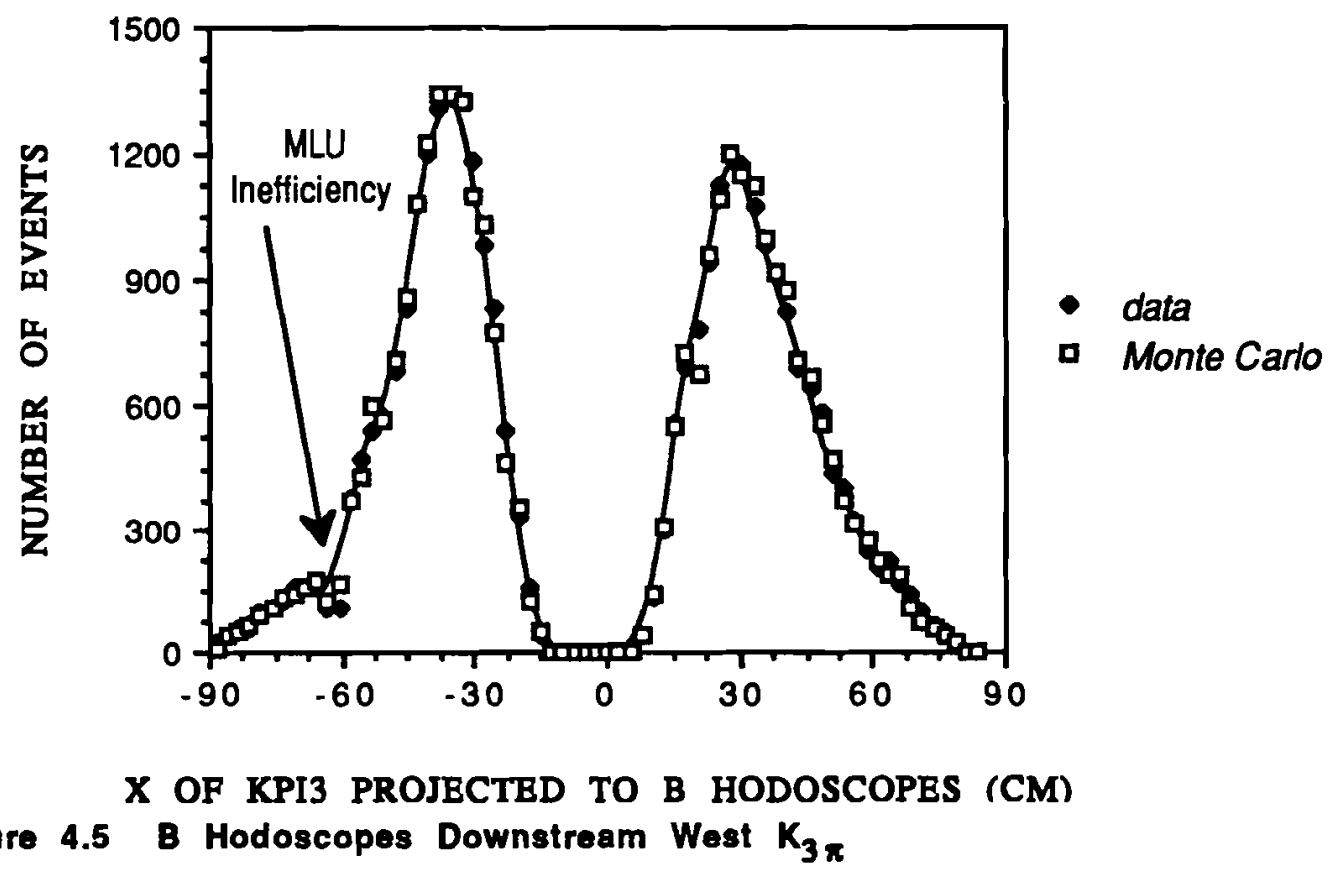


The second level MLU trigger was $99 \%$ efficient. Requiring only one hit in each bank of each hodoscope array lost $45 \%$ of both the $\mathrm{K} \rightarrow 3 \pi$ and $\mathrm{K} \rightarrow 2 \pi$ decays due to $\mathrm{A}$ and $B$ hodoscope inefficiencies and accidentals. This corresponds to an average accidentalinefficiency loss of less than 3\% per hodoscope element. Of this $3 \%$ loss per element, generally $1 \%$ corresponded to hodoscope inefficiencies and $2 \%$ corresponded to accidentals/noise

The average number of adjacent noise hits for both arrays was calculated also. The MLU trigger processor chose the inner element for $A$ hodoscope hits and the outter element for $B$ hodoscope hits when determining the slope of the tracks if a neighboring element were hit. For the $\mathrm{A}$ hodoscopes $3.1 \%$ of the time there was an adjacent hit to the outer side and $2.6 \%$ of the time to the inner side of a track. For the $B$ hodoscopes these numbers were $3.7 \%$ and $2.8 \%$ respectively.

\subsection{Charged Momentum Ratio (CMR) Trigger Processor}

\subsubsection{Introduction}

This trigger processor used the information from the two chambers downstream of the analysis magnets $\mathrm{C} 4$ and $\mathrm{C5}$ to give a refined measurement of the ratio of the momenta of the two charged tracks. As in the MLU trigger processor, two levels of CMR processing were used. The first level encoded the chamber hit addresses of groups of 4 wires ( $8 \mathrm{~mm}$ wide) from each of the two sides of the two chambers $\mathrm{C} 4$ and $\mathrm{C5}$, and the second level combined these four addresses in order to measure the slope ratio of the tracks. Depending on the ratio of the slopes, the CMR trigger processor identified the parent particle as a kaon, lambda or anti-lambda.

The CMR trigger processor used Emitter Coupled Logic (ECL) series MECL10000 electronics to process an event in $\sim 120$ nsec. The layout of the CMR trigger processor is shown in Figure 4.6. It is seperated into two parts, the first level near-beam apparatus and the second level control room apparatus.

\subsubsection{Design : First Level CMR trigger processor}

The first level of the CMR used the wire positions from chambers C4 and C5. The outermost hit in each half of each chamber was converted to binary and sent to the the Arithmetic Logic Unit in the control room 70 meters away. The Aritmetic Logic Unit (ALU) was the main portion of the second level CMR trigger processor. 


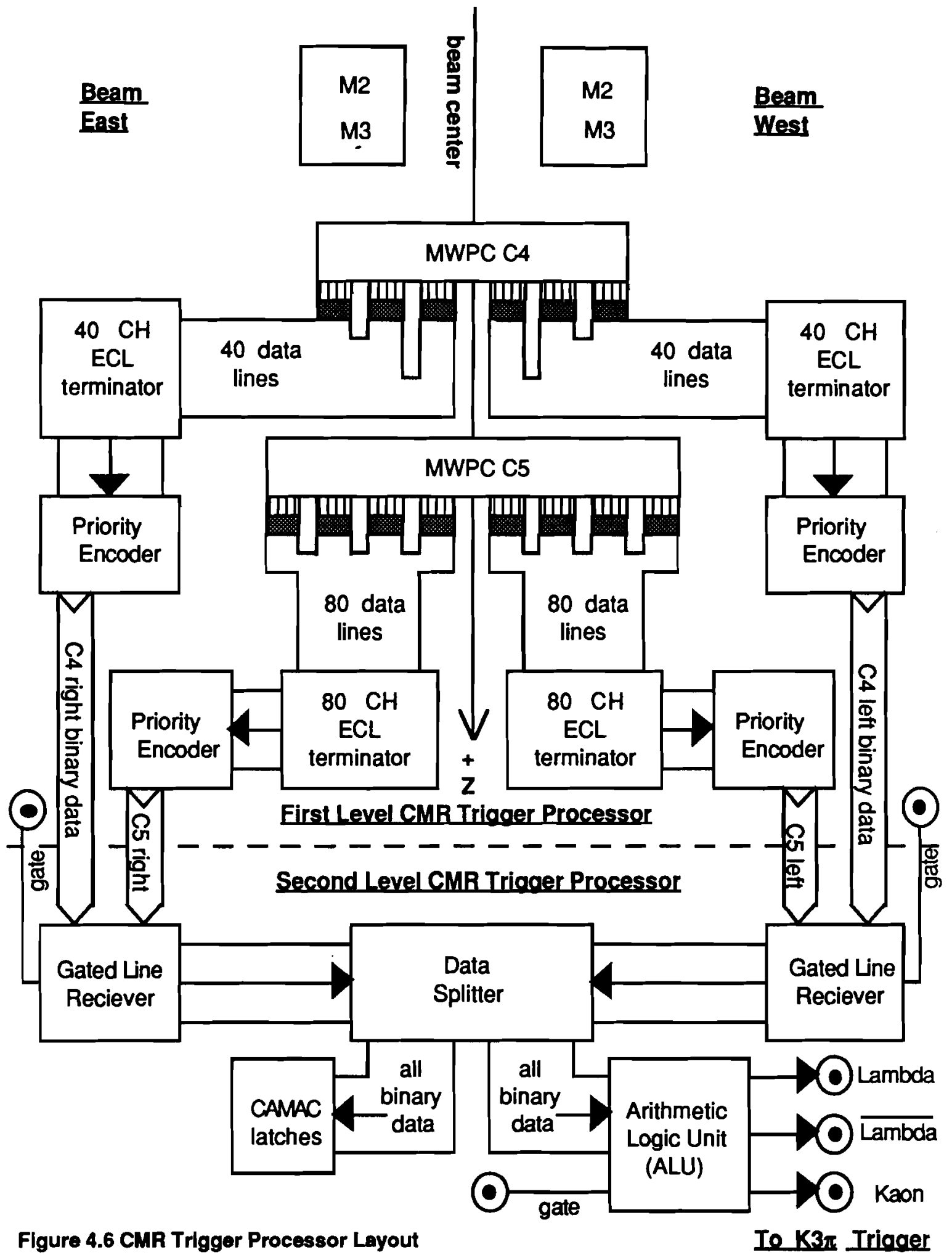


The center line for the CMR trigger processor was defined as the physical center of each of the two chambers C4 and C5. Chamber C4 (C5) had 320 (640) sense wires in $\mathrm{x}$ with $2 \mathrm{~mm}$ spacing with each amplifier card on the chamber servicing four sense wires. Thus MWPC C4 was 80 amplifier cards wide and the second MWPC used by the CMR C5, was 160 amplifier cards wide in $x$. Each amplifier card generated a fast-or pulse of the amplifier card's four sense wires. This pulse was transmitted to a "motherboard" which supported the amplifier cards. The CMR tapped into the motherboard to get coordinate information from the fast-or pulses. To suppress electronic noise and jitter, the signals were stretched to $100 \mathrm{~ns}$ using "one-shot's" and then were sent as bipolar signals through a ground shielded twisted pair cable to a terminator circuit. Each side of the twisted pair cable was passed through a ferrite bead. The terminator circuit sent the data to the Priority Ecoder shown in Figure 4.7 and described below.

The Priority Encoder (PE) circuit chose the hit most distant from the chamber center line on each side of the two chambers. This hit selection was chosen because the neutral beam went through the center of each chamber causing the majority of accidental hits to be near the center of the chamber. The PE circuits consisted of MC10165 priority encoder chips and MC10164 multiplexer chips. The MC10165 has 8 inputs and the three bit address of the least significant input line with a signal is encoded on the $A, B$ and $C$ outputs. For the CMR trigger processor the address of the most significant input line with a signal was desired and thus the order of the input lines to each encoder was reversed. The D output of the MC10165 was used as a data present line as it went high if any of the 8 inputs were high. One MC10165 collected all the $D$ lines and encoded the address of the highest priority active $D$ line which corresponded to the three most significant bits (MSB) of the address of the outermost hit card. These three bits also identified which of the five priority encoder chips had the address (the three least significant bits (LSB)) of the outermost hit address.

Since the inputs to all the priority encoder chips were reversed, the output was the inverse of the hit address. Thus the complements of the three LSB and the three MSB formed the address of the outermost active card. 
Chip List:

1-6 MC10165

7-9 MC10164

Notes:

All outputs have a1kohm resitor

to -5.2 volts
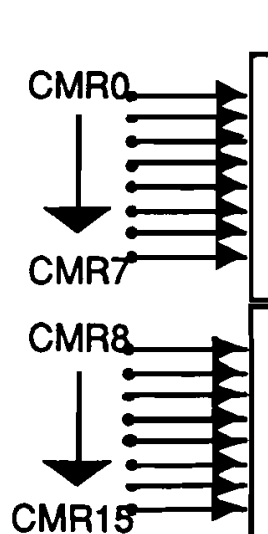

|우

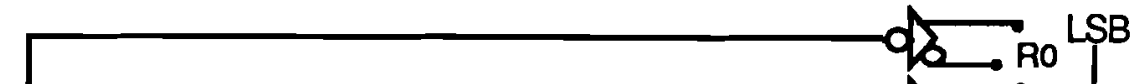
$d \sqrt{2} \mathrm{R} 1$ (n)

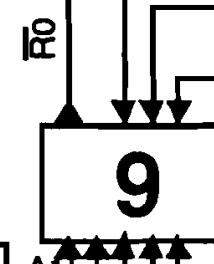

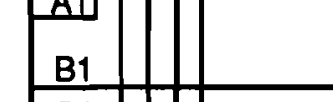
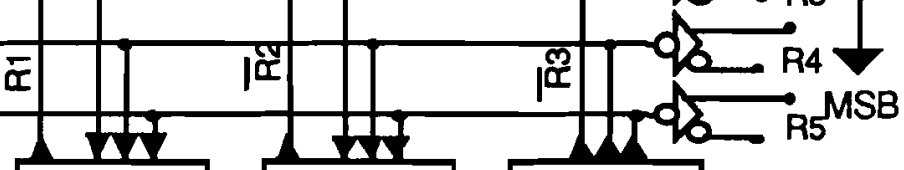

1<smiles></smiles>

2

$\mathrm{B} 2$

\begin{tabular}{|l|lll}
\hline $\mathrm{C} 2$ & & \\
\hline $\mathrm{D} 2$ & & & \\
\hline
\end{tabular}

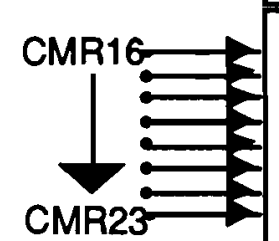

A3

3

$B 3$

$\mathrm{C} 3$

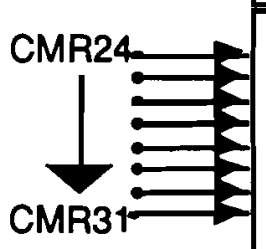

CMR32<smiles></smiles>

5

B5

C5

Figure 4.7 Priorlty Encoder 
Chamber C4 needed two 40 channel encoder circuits to address the 80 cards (320 wires) in C4. Each encoder chip had five 8 channel MC10165's to encode the three LSB of the card adresses and one more to encode the three MSB of the outermost card in order to address 40 cards. For chamber C5 two 80 channel encoder circuits were needed to define the address of the 640 wires or 160 cards in C5. Thus one had to address $10 \mathrm{MC10165's} \mathrm{with} \mathrm{only} \mathrm{three} \mathrm{bits.} \mathrm{To} \mathrm{solve} \mathrm{this} \mathrm{problem} \mathrm{the} \mathrm{two}$ MC10165's receiving data from the outermost cards were connected to the first two MC10165's. This didn't lose any data since only the outermost hits were used in the CMR trigger processor. AND gates were used to inhibit the outputs of the two MC01065's receiving data from the innermost cards and the first $8 \mathrm{D}$ lines when data was present on the two MC10165's receiving data from the outermost cards. This inhibit signal was then used to form the $7^{\text {th }}$ bit of the final answer in order to obtain the correct address.

The four addresses of the outermost hits in each side of chambers C5 and C6 from the four PE circuits were then sent through differential twisted pair cables to the Arithmetic Logic Unit in the control room. The time required for the first level operation was less than 50 ns.

\subsubsection{Design : Second Level CMR Trigger Electronics}

The four addresses, $\mathrm{C5}_{\text {left }}, \mathrm{C} 4_{\text {left }}, \mathrm{C} 4_{\text {right }}$ and $\mathrm{C} 5_{\text {right }}$, from the first level PE circuits were latched in the control room and sent to the second level of the CMR which consisted of the Arithmetic Logic Unit (ALU) which calculated the slopes. This information was then fed into the $\mathrm{K}^{\circ} \rightarrow 3 \pi$ trigger. The schematic of the ALU is shown in Figure 4.8.

In determining the slopes it was assumed that the two left and right hits were made by the same particle. In practice very few particles actually crossed in between these two chambers and it never happened for $\mathrm{K}^{\circ} \rightarrow 3 \pi$ decays. The algorithm used to calculate each slope was:

$$
\begin{aligned}
& \text { slope }_{\text {left }} \alpha \mathrm{C5}_{\text {left }}-\mathrm{C}^{\mathrm{C}} 4_{\text {left }} \\
& \text { slope }_{\text {right }} \alpha \mathrm{C5}_{\text {right }}-\mathrm{C}_{\text {right }} .
\end{aligned}
$$

since the momentum of the tracks is proportional to the slope and the $z$ distance between the chambers $\mathrm{C} 4$ and $\mathrm{C} 5$ is a constant for all events. Then the left and right slopes were compared to ensure that one slope was not greater than three timse the 
other.

In order to compare the slopes the ALU performed four operations. First two subtractions formed the left and right slopes $m_{R}$ and $m_{L} \cdot m_{R}=(5 R+\overline{4 R})$ and $m_{L}=$ $(5 L+\overline{4 L})$ where $5 R, 5 L, 4 R$ and $4 L$ are the addresses of the outermost hits of the right and left sides of chambers C5 and C4. Each subtraction used MC10181's which are 4 bit arithmatic logic chips that were wired for addition.

The second operation shifted $m_{R}$ and $m_{L}$ by one bit forming $m_{R} / 2$ and $m_{L} / 2$. And then additions were performed using two cascaded MC10181's yeilding $3 \mathrm{~m}_{\mathrm{R}} / 2=$ $m_{R}+m_{R} / 2$ and $3 m_{L} / 2=m_{L}+m_{L} / 2$. The fourth and final process tested for $\left(m_{R} / 2\right.$ $\left.>3 m_{L} / 2\right)$ or $\left(m_{L} / 2>3 m_{A} / 2\right)$ using the 5-bit magnitude comparitor MC10166. The four comparitor outputs (labeled Ro, R1, R2, and R3 in Figure 4.8) yeilded the following possible situations:

\begin{tabular}{|c|c|c|c|c|}
\hline R3 & $\mathrm{R} 2$ & R1 & Ro & CONDITION \\
\hline $\mathbf{L}$ & $\mathrm{H}$ & $\mathbf{L}$ & $\mathbf{L}$ & $3 m_{L^{\prime}} / 2=m_{R} / 2$ \\
\hline $\mathbf{L}$ & $\mathbf{L}$ & $L$ & $\mathrm{H}$ & $3 m_{R^{\prime}} / 2=m_{L} / 2$ \\
\hline $\mathbf{L}$ & $\mathrm{H}$ & $\mathrm{H}$ & $\mathbf{L}$ & $3 m_{L} / 2<m_{R} / 2$ \\
\hline $\mathrm{H}$ & $\mathbf{L}$ & $\mathbf{L}$ & $\mathrm{H}$ & $3 m_{\mathrm{R}} / 2<m_{\mathrm{L}} / 2$ \\
\hline $\mathbf{L}$ & $\mathrm{H}$ & $\mathbf{L}$ & $\mathrm{H}$ & $3 m_{L} / 2>m_{R} / 2 \quad$ AND $\quad 3 m_{R} / 2>m_{L} / 2$ \\
\hline
\end{tabular}

Table 4.2 CMR Trigger Comparltor Output Conditlons

If the slopes were less than or equal to 3 then R1 and R3 were low. Thus the NOR of $\mathrm{R} 1$ and $\mathrm{R} 3$ formed the $\mathrm{K}^{\circ}$ bit. The parent particle was identified as a lambda or an anti-lambda if R1 was high and R3 low, or R1 low and R3 high respectively. Finally a MC10192 quadruple bus driver was used to convert the signals to NIM levels $(0.0 v \rightarrow-0.8 v)$. This signal was then used in the $K^{0} \rightarrow 3 \pi$ trigger.

\subsubsection{CMR Trigger Processor Efficiencies}

I determined the CMR efficiencies in the same way as the MLU efficiencies described in Section 4.3.4 using $V$ trigger data. A program which simulated the CMR trigger predicted that $95 \%$ of the lambdas would be removed from a vee topology 
60

Left Side Data BIts: address of outermost left side hit

Note All outputs have a 1 kohm

resistor to $-5.2 \mathrm{~V}$

ก 0 in $\mathrm{C} 4 \& \mathrm{C5}$

$\approx+\frac{2 G}{5}+\frac{\pi}{0}$

$\operatorname{high}(\mathrm{H})=-0.8 \mathrm{~V}$

low $(L)=-1.6 \mathrm{~V}$

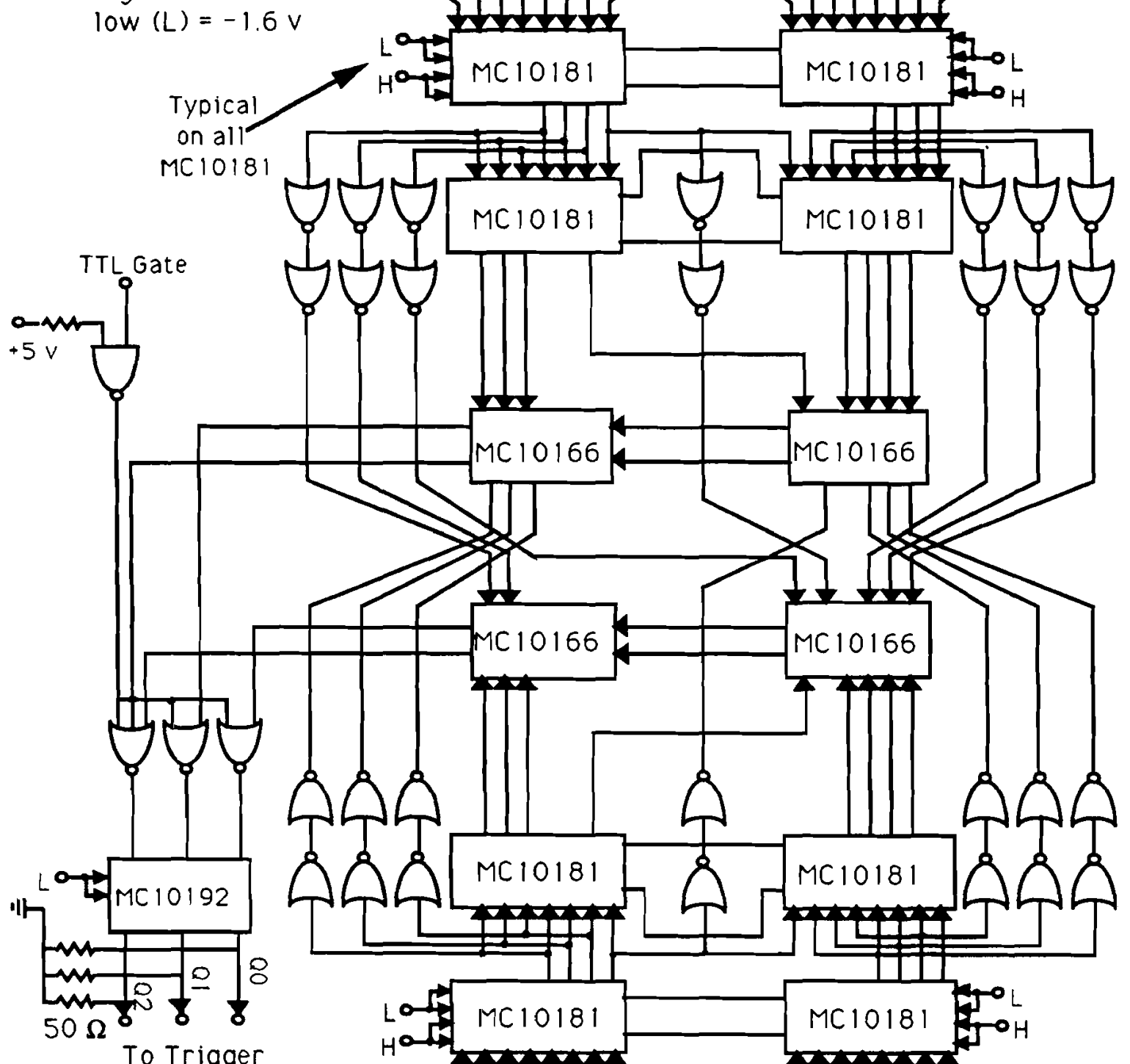

To Trigger
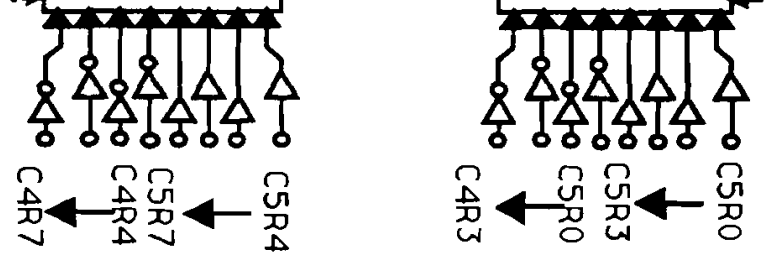

Right Side Data Bits: address of outermost left side hit in $C 4 \&$ C.

Figure 4.8 Arlthmetlc Loglc Unlt (ALU) 
trigger as would $5 \%$ of the possible $\mathrm{K}^{0} \rightarrow \pi^{+} \pi^{-} \pi^{0}$ candidates. The CMR trigger apparatus (chambers and trigger processor) actually removed $81 \%$ of the lambdas and $55 \%$ of the $\mathrm{K}^{\circ} \rightarrow \pi^{+} \pi^{-} \pi^{\circ}$ candidates due to chamber and individual channel inefficiencies which are discussed later in this section.

A computer program simulated the CMR operation using the latched chamber wire hit information to determine the outermost hit card address. The addresses determined by the first level CMR trigger were latched and thus could be compared to those determined by the CMR simulation program. For a given channel, if the simulation program found it to be the outermost hit channel and the CMR did not, an inefficincy was recorded. I created a file of efficiencies for each channel in the trigger processor for chambers $\mathrm{C} 4$ and $\mathrm{C} 5$. I discovered that card 48 on the east side of $\mathrm{C5}$ was either dead or $94 \%$ efficient, jumping on and off tape by tape. Figure 4.9 shows the $x$ position of the charged tracks at $\mathrm{C5}$ for Monte Carlo and data after adding the CMR channel efficiencies to the Monte Carlo program. The inefficient channel is marked on the plot. The other dip in the plot at $-40 \mathrm{~cm}$ corresponds to the MLU inefficient channel discussed in 4.3.4.

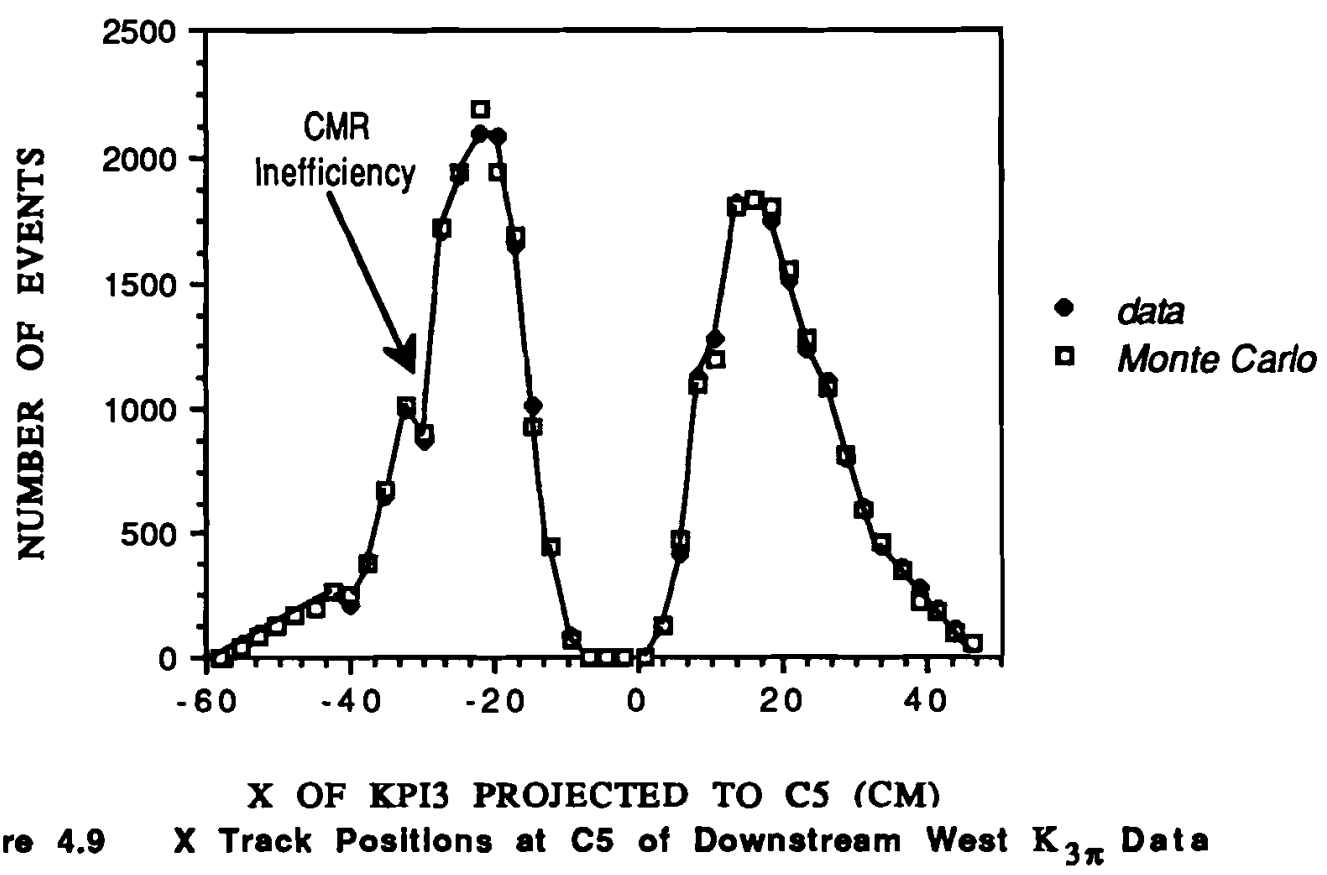


The average channel efficiency of the first level CMR trigger processor was $91 \%$. Much of this inefficiency probably came from the difference between the chamber and the first level CMR gate widths. The chamber gate width was $150 \mathrm{nsec}$ or $\sim 30 \%$ wider than the CMR gate width and thus events later than the CMR $100 \mathrm{nsec}$ gate would not be latched by the CMR but would be latched by the chamber latches. The second level CMR trigger processor was $96 \%$ efficient, not including the inefficiencies of the chambers $\mathrm{C}_{4}$ and $\mathrm{C} 5$. The $\mathrm{K} \rightarrow 3 \pi$ Monte Carlo simulation program showed that $29 \%$ of $\mathrm{K} \rightarrow 3 \pi$ decays were lost due to the inefficiencies in chambers $\mathrm{C} 5$ and $\mathrm{C} 6$. Thus of the $55 \%$ of $\mathrm{K} \rightarrow 3 \pi$ decays lost in the CMR trigger apparatus, $29 \%$ were lost in the chambers and $26 \%$ were lost in the CMR electronics.

\subsection{Glass Cluster Finding (GCF) Trigger}

\subsubsection{Introduction}

The GCF trigger was a Transistor Transistor Logic (TTL) circuit which identified and counted neutral energy clusters in the lead glass array. It was designed to determine that there were at least two showers in the lead glass array which were coincident with a neutral decay trigger and not associated with hits in the $\mathrm{Pi}$ hodoscope array. The timing of the lead glass shower information had to be kept as tight as possible to help reject the remaining trigger background of $\mathrm{K}^{0} \rightarrow 2 \pi$ decays with two unassociated random photon showers.

An electromagnetic shower in the lead glass array typically spread energy over a few blocks. A "cluster" was defined as a contiguous group of blocks, each of which had an output signal from its photomultiplier tube above threshold. The Pi hodoscope array signals were used by the GCF trigger to identify which clusters were initiated by charged particles. The GCF trigger then determined the number of neutral clusters there were in the lead glass array.

The trigger logic looked for cluster edges, defined as a hit block with three unhit neighbors as shown in Figure 4.15. In the $\mathrm{K}^{\circ} \rightarrow 3 \pi$ trigger it searched for two or more neutral clusters thereby cutting $\mathrm{K}^{\circ} \rightarrow 2 \pi$ events from the $\mathrm{K}^{\circ} \rightarrow 3 \pi$ trigger. It processed an event in approximately $100 \mathrm{nsec}$.

The GCF trigger processor did not use the outer edge blocks in the lead glass array because there were few photons from the $\mathrm{K}^{\circ} \rightarrow 3 \pi$ decay in this region, and the 
resolution would be very poor due to shower leakage from the edge blocks. The 52 lead glass block signals went into discriminators and then on to the GCF trigger processor. The discriminators had $25 \mathrm{mv}(\sim 2 \mathrm{GeV})$ thresholds and output a $20 \mathrm{~ns}$ wide pulse (NIM levels). The threshold was set relatively high to omit blocks with small pulses. This was done since the GCF trigger processor looks for edges of a cluster and if smaller hits are allowed it is more likely that two clusters will coalesce.

The Pi hodoscopes' signals passed through a discriminator with a $25 \mathrm{mv}$ threshold and a 60ns wide output pulse. These signals then went on to the GCF trigger processor. The ten vertical and six horizontal hodoscopes formed thirty distinct regions of overlap, shown in Figure 3.5, which were used to veto the blocks in the glass array behind the overlap region when a coincidence occured.

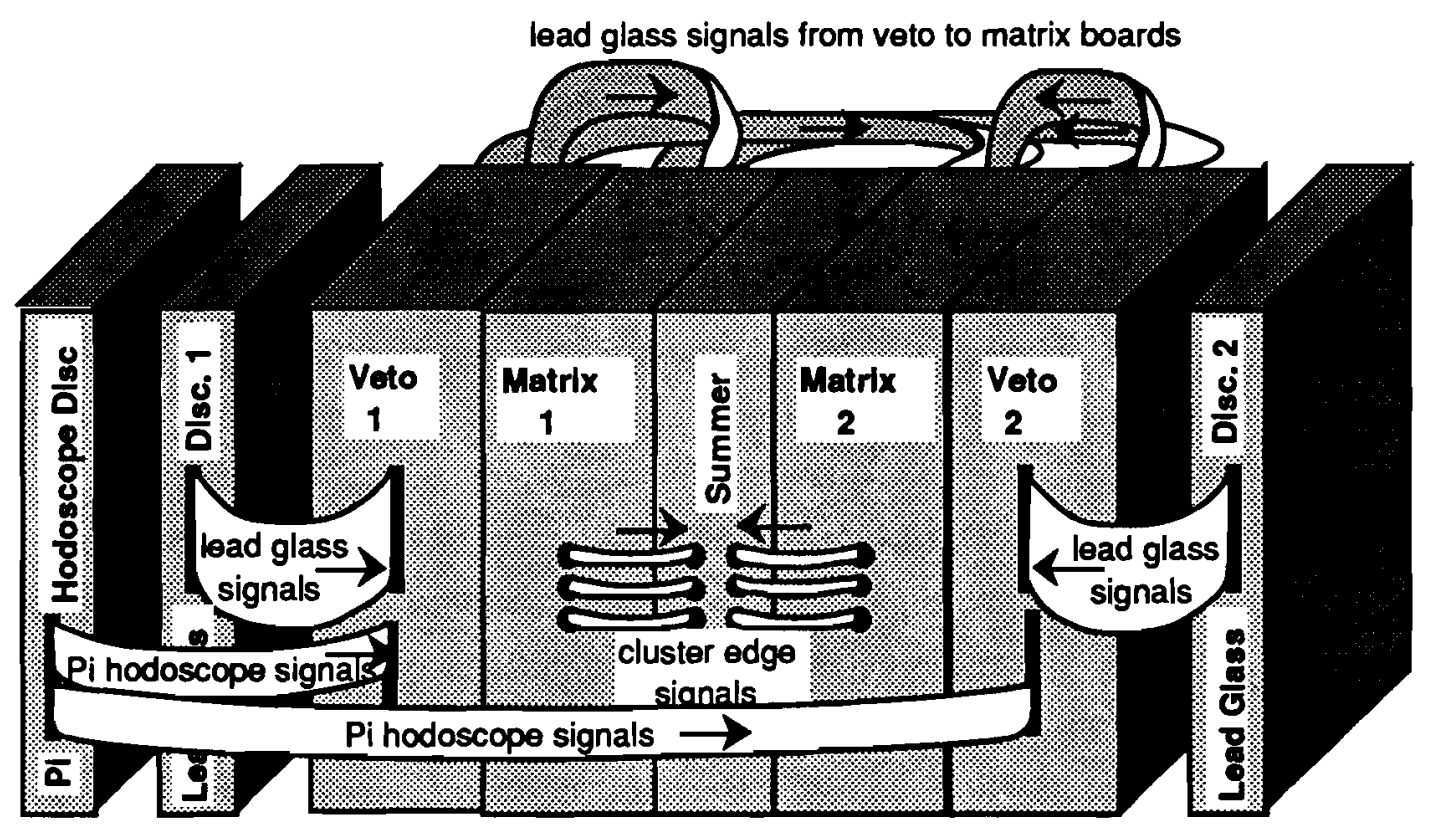

Figure 4.10 GCF Trigger Processor Boards and Thelr Signals

The GCF trigger processor consisted of five TTL circuit boards. Figure 4.10 shows the five boards that made up the GCF trigger processor and how they were connected. The lead glass array was divided electronically into two halves as shown in Figure 4.11, of 26 blocks each. The electronics for each half were connected with twisted pair cables in order to identify those clusters which spanned the center of the 
64

array. The Veto Boards (one for the left side and one for the right) dealt with the $\mathrm{Pi}$ hodoscope vetoing of charged clusters and the two Matrix Boards (left and right) looked for edges of the remaining clusters. The results of the searches for either side were then summed in the fifth board, the Summer Board.

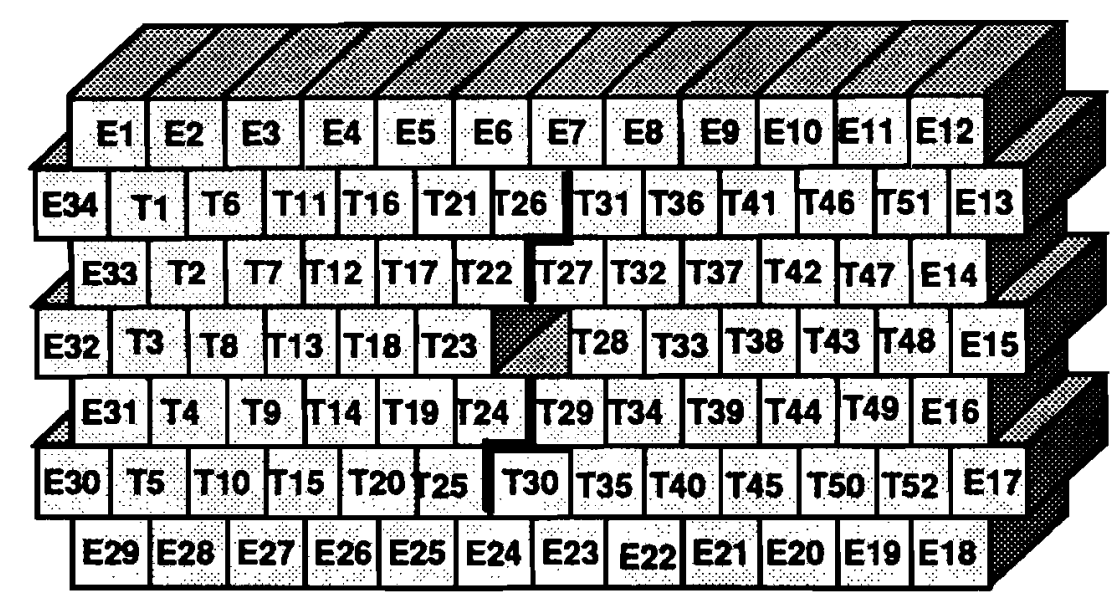

Note:

En are edge blocks not connected to the GCF

Tn are blocks connected to GCF

- is division of two halves of array for GCF

Figure 4.11 GCF Trigger Processor Lead Glass Numbering

\subsubsection{Design : Veto Boards:}

The eight Pi hodoscope signals and 26 lead glass block signals from half of the array were sent to each veto board from the discriminators. The hodoscope signals were $60 \mathrm{nsec}$ wide to veto the $20 \mathrm{nsec}$ wide lead glass block pulses. Veto Board 1's circuit diagram is shown in Figure 4.12. Figure 4.12a is the first portion of the veto board which forms the Pi hodoscope overlap signals. Figure $4.12 \mathrm{~b}$ is the second portion of the veto board where the lead glass block signals are vetoed by the $\mathrm{Pi}$ hodoscope overlap signals.

In the first portion of the veto boards the discriminated Pi hodoscope signals went through MC10125 ECL to TTL converters. The next stage was 74ALS00 quad 2-input NAND gates which each had a horizontal and a vertical Pi hodoscope signal as inputs. The output was an inverted pulse if overlapping signals were found. Figure 4.13 shows the two cases for the Pi hodoscope overlaps and the lead glass block 
signals that they veto.

The second portion of the veto boards actually vetoed the lead glass block signals. Since the Pi vetoes only covered the middle three rows of lead glass blocks, any given lead glass block could be vetoed by at most 6 possible hodoscope overlaps. Each of the possible veto overlaps for a given block were input to 74ALS30 single 8 input NAND gates. The outgoing positive pulse from these gates was used to veto the inverted lead glass block pulse from the 74ALS04 hex inverter at a 74ALS27 3input NOR. The third input of the NOR gate was an unconditional lead glass block veto shown in Figure 4.14.

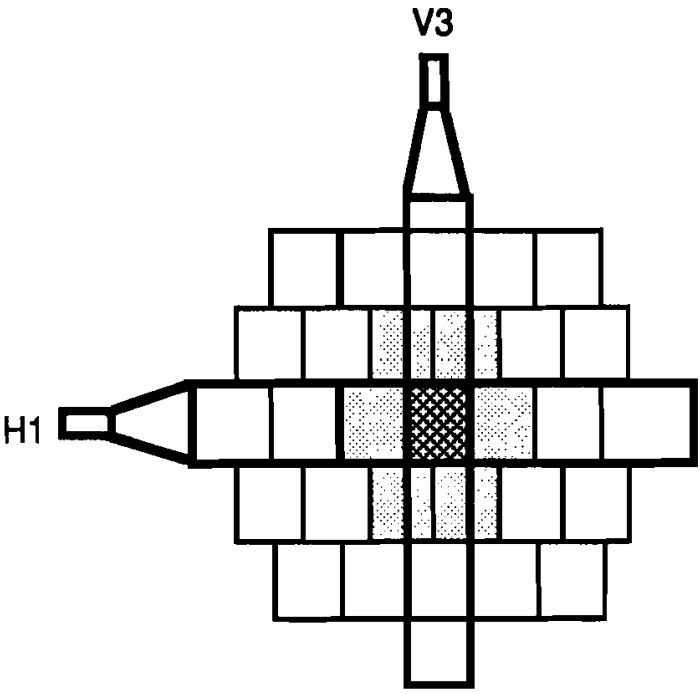

V3, H1 hodoscope hits veto 7 blocks

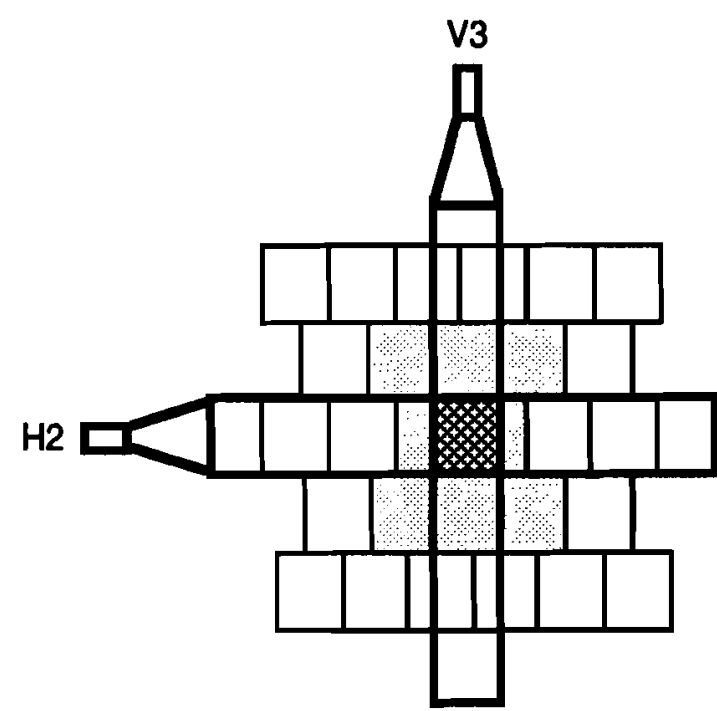

V3, H2 hodoscope hits veto 8 blocks

Overlap Region of Pis

Blocks vetoed

Figure 4.13 PI Hodoscope Overlap Vetoes

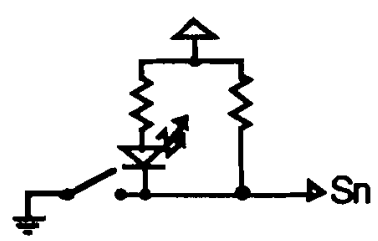

Figure 4.14 Unconditional Lead Glass Block Veto 
Note:

$\mathrm{HVn}$ is the signal from the horizontal Pi hodoscope Hn $\mathrm{V} \mathrm{n}$ is the signal from the vertical $\mathrm{Pi}$ hodoscope $\mathrm{Vn}$

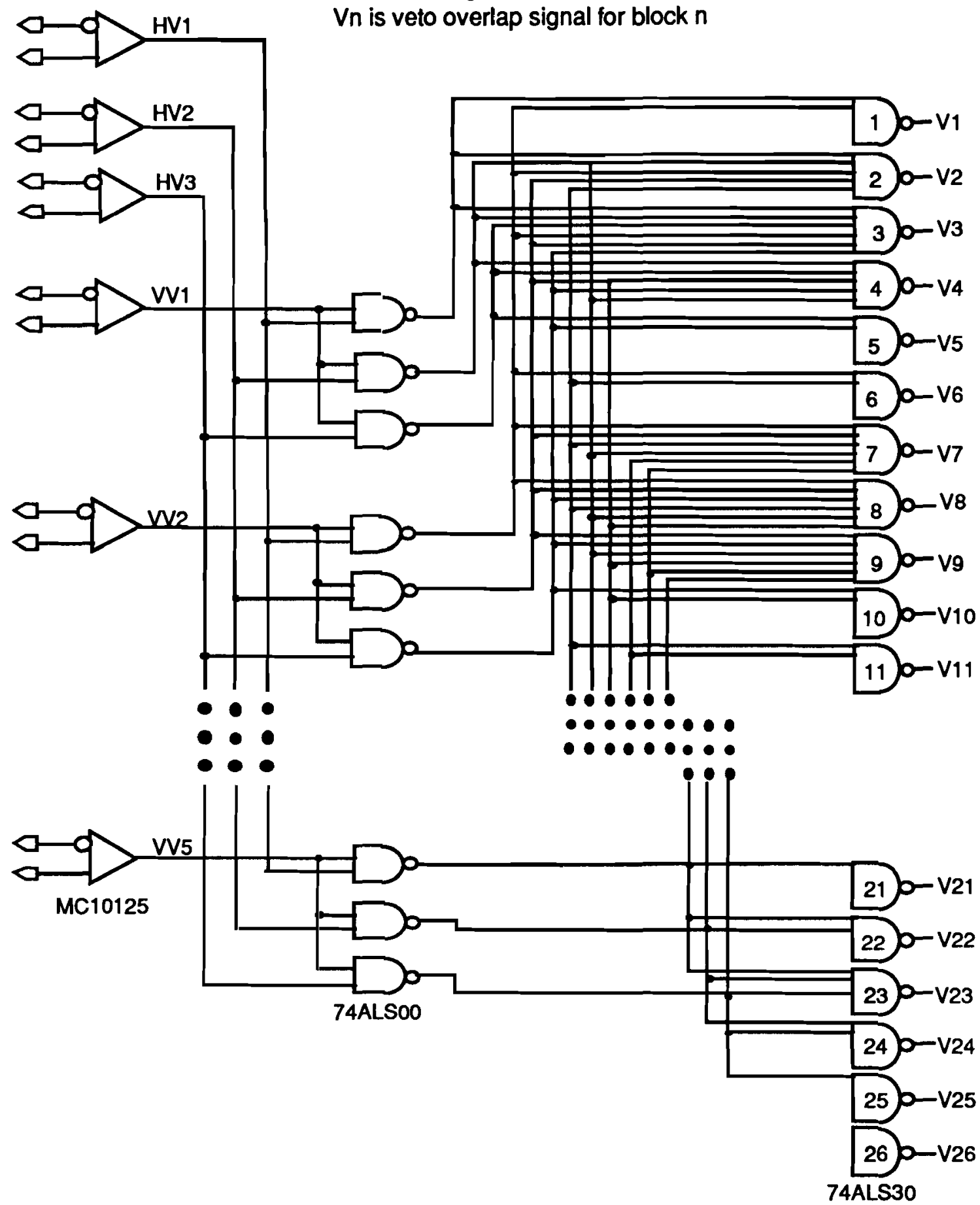

Flgure 4.12a Veto Board 1: PI Hodoscope Overlap Signal Information 


\section{Note:}

$\mathrm{V} n$ is veto signal for block $\mathrm{n}$ from Pi overlaps

$\mathrm{S} n$ is unconditional block veto for block $n$

Tnx is block n's signal to be sent to Matrix Board 2

$\mathrm{CHn}$ is block n's signal from the discriminator
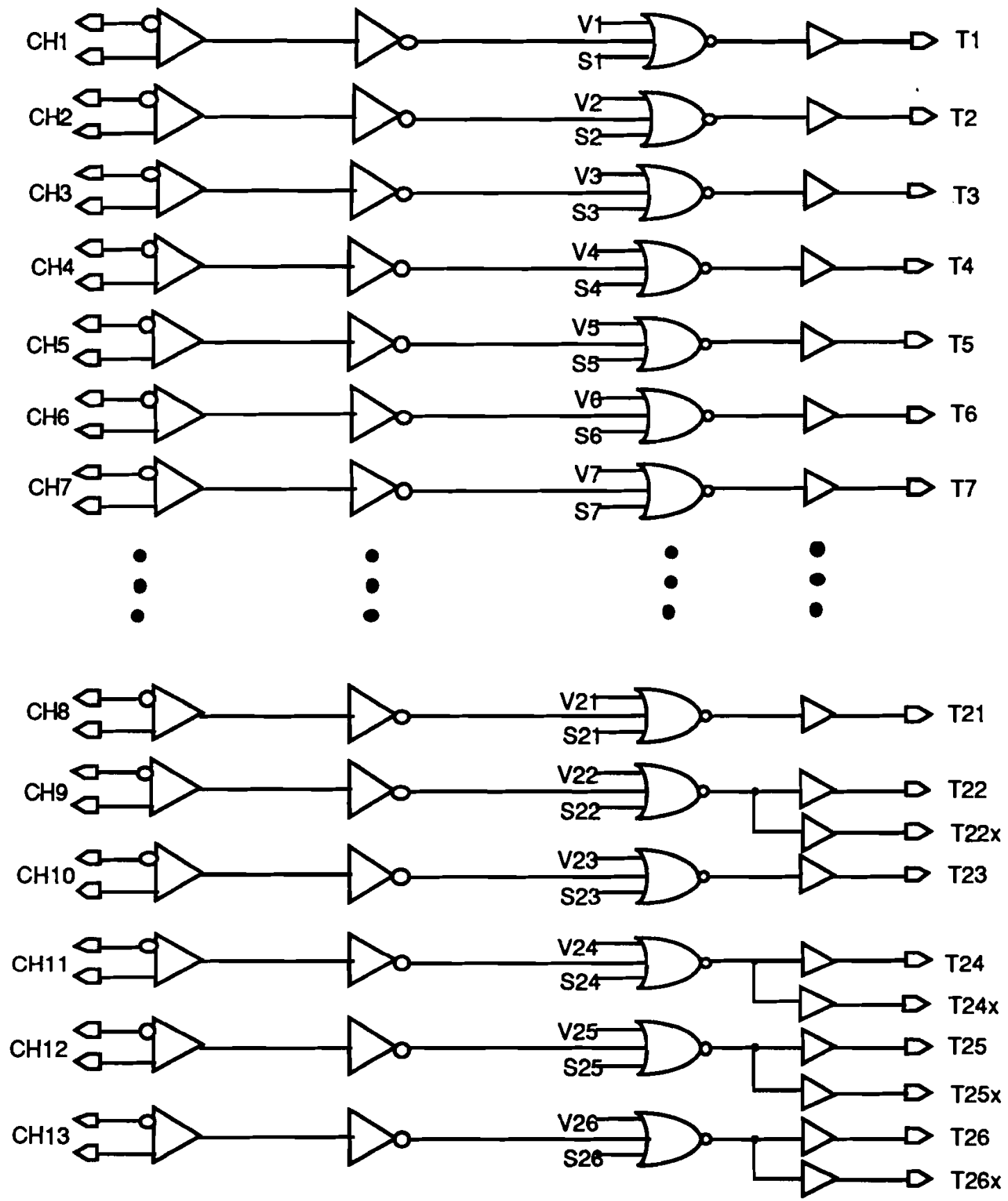

Figure 4.12b Veto Board 1: PI Hodoscope Overlap Signal Information 
These unconditional lead glass block veto circuits were connected to 26 switches on the front of each veto board. Thus one could veto any lead glass block desired with these switches. This was very useful during the testing phases of the GCF trigger processor.

The resulting pulse from the NOR gate was then sent on to the matrix boards through a 74S244 driver. Only lead glass block signals which were not vetoed by the $\mathrm{Pi}$ hodoscope overlap signals were present at this stage. Lead glass blocks in the center region of the array, i.e. T24,T25,T29,T30 etc. in Figure 4.11, sent their signals to both matrix boards (corresponding to the signals T24x, T25x etc in Figure 4.12b).

Two test points were available on the veto boards. One was essentially a NOR of all the output pulses (non-vetoed lead glass block pulses), and the other was an OR of all the veto pulses from the Pi hodoscope overlaps. These two test points were used for timing the $\mathrm{Pi}$ hodoscope-veto 60ns pulses to overlap the 20ns lead glass block pulses with $\sim 20$ nsec leeway on either side.

\subsubsection{Design : Matrix Boards}

Cluster identification involved the use of an image processing technique called an "edge-finding algorithm". This technique uses the fact that a cluster is defined not so much by its size as by its edges. One determines if a block is an "edge" of a cluster by looking at its neighboring blocks' signals. The three algorithms of Figure 4.15 were chosen to determine the cluster edges. They had to be implemented in parallel in order to rapidly find clusters. The final number of clusters was defined as the minimum of the output of the three seperate algorithms or the miminum number of edges found in the three directions.

The matrix boards looked for cluster edges from three different directions and sent the sum of the number of edges found in each direction on to the summer board. The algorithm is shown in Figure 4.15 where N1 through N6 are the nearest neighbors of a lead glass block with a signal. Edges were searched for in the three directions shown in Figure 4.15.

The schematic of one of the Matrix boards is shown in Figure 4.16. The first stage of the Matrix boards sent the lead glass block signals through a 74S244 driver. The pulse was then split into three. One signal was inverted and input to a 74508 
AND gate along with the original signal. This clipped the last few nanoseconds off the original pulse cleaning up the timing and trimming down the signal to 15-20ns. The other signal was inverted in a $74 \mathrm{SO} 4$ and sent to neighboring blocks' $74 \mathrm{H} 21$ AND gates to determine cluster edges.
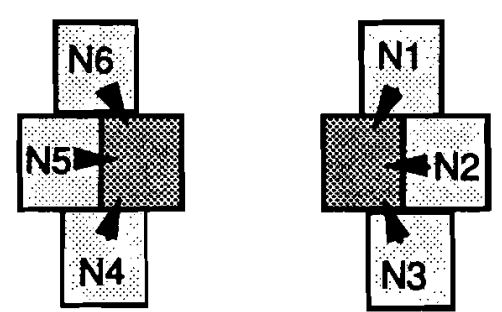

Hit Block

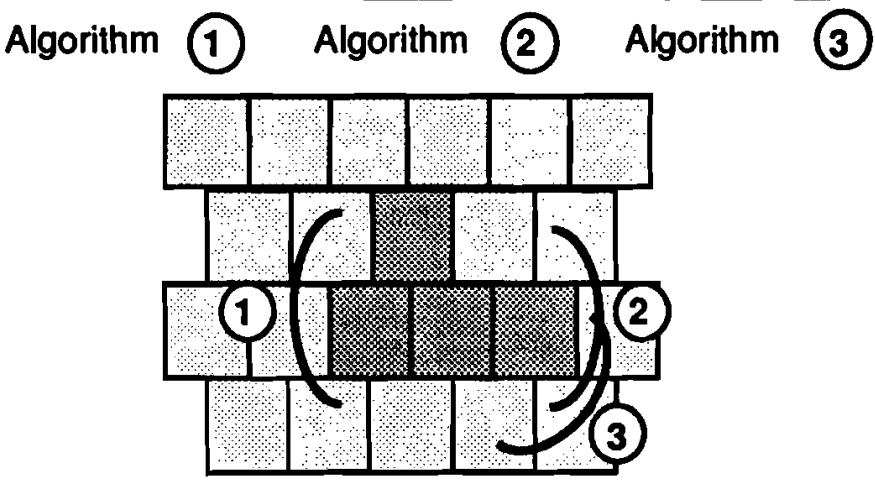

Figure 4.15 GCF Trigger Processor Lead Glass Block Algorlthm

Each column of 74H21's tested one of the three algorithms of Figure 4.15. For example the first column of AND gates determined if any of a hit lead glass block's three left neighbors also had a signal. If no neighbor were hit, a cluster edge was found. This left neighbor scan was performed on all lead glass blocks in the array and only when a hit lead glass block's three left neighbors had no signal present was a positive pulse sent out of the AND gate in column one. The same process ocurred in parallel in column 2 and 3 for the other two algorithms. A positive pulse out of the $74 \mathrm{H} 21$ turned on a $2 \mathrm{~N} 3904$ transistor. These emitter-coupled transistors were wire OR'ed. The OR'ed pulse height was proportional to the number of coincident positive pulses out of the $74 \mathrm{H} 21$ AND gates and thus proportional to the number of edges found using a given algorithm. The signal from each of the three algorithms traveled on lemo cables to the Summer Board. 


\section{0}

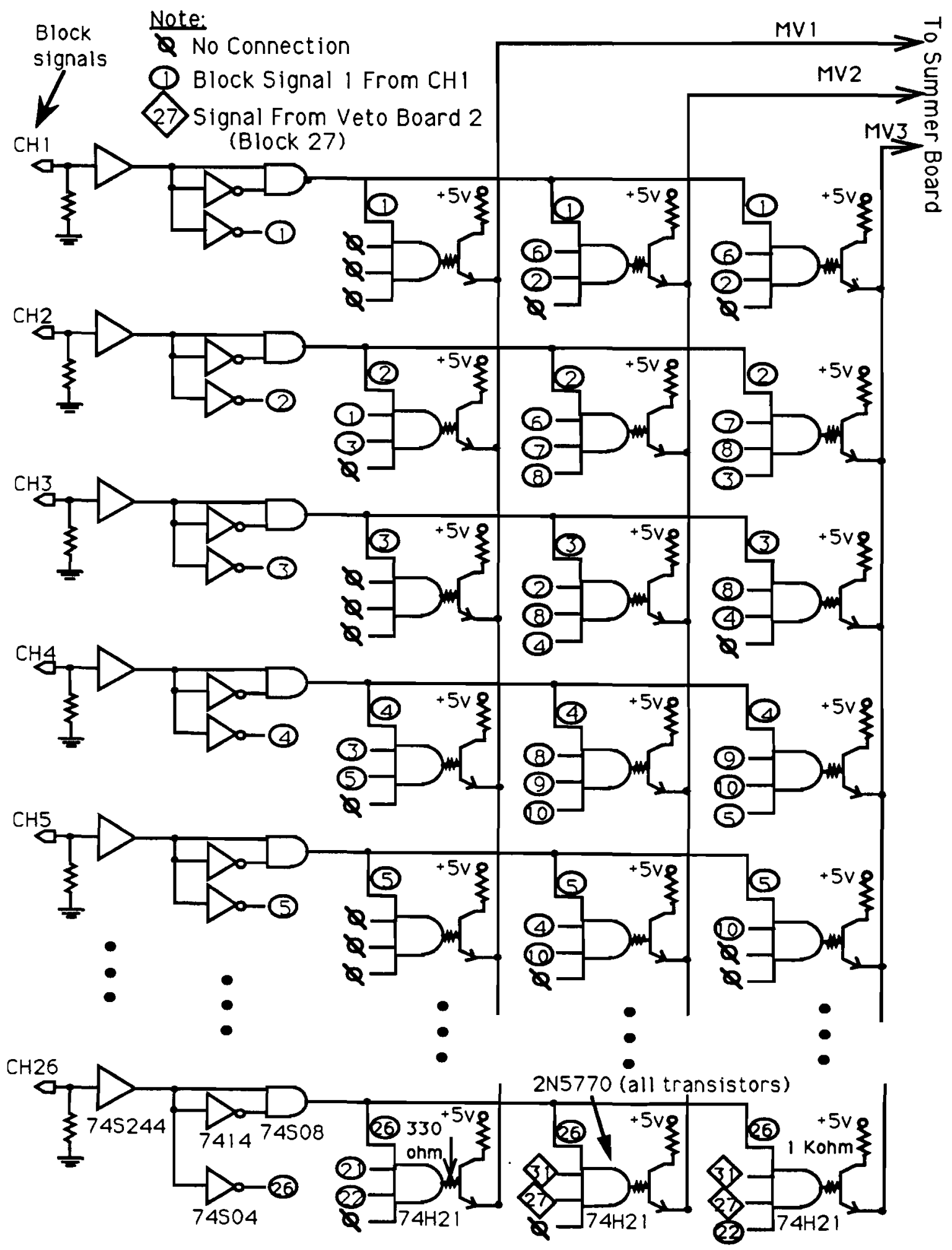

Figure 4.16 Matrlx Board 1: Finding Cluster Edges 


\subsubsection{Design : Summer Board}

The Summer Board, shown in Figure 4.17, found the number of energy clusters in the lead glass array, defined as the minimum number of edges from any of the three algorithms. Signals MV1, MV2 and MV3, the results of the Matrix boards, were sent to AM687 comparators. Each comparator compares the input pulse height to two preset levels. The MV signals were checked in parallel to see if they corresponded to greater than $0,1,2$ or 3 cluster edges by comparing their pulse heights to the thresholds set by the $2 \mathrm{~K} \Omega 20$ turn potentiometers. These thresholds were set using a pulse generator to strobe various lead glass block combinations and then observing the output. The signal height from the transistors in the matrix board varied somewhat with the input block signals. To minimize the loss of events due to these channel variations, the smallest MV signal from two single lead glass block hits and the largest MV signal from a single lead glass block hit were used to set the two cluster thresholds accurately.

The signals from the AM687 comparators were converted to TTL in MC10125 ECL to TTL converters. The 74ALS20 3 input NAND gates determined how many edges were found in each of the three scans. One NAND gate checked for at least one edge, the second NAND gate for at least two edges and the third for more than two. Only the $\geq 2$ output was used in the $\mathrm{K}_{3 \pi}$ trigger. If at least two cluster edges were found by each algorithm, the 2 N3940 transistor corresponding to $\geq 2$ turned on giving a NIM pulse as an ouput. This signal was used as part of the $\mathrm{K}_{3 \pi}$ trigger. The AM687s' thresholds were periodically checked and set such that very few two cluster signals would be construed as only single cluster events and thus single clusters sometimes satisfied the $\geq 2$ (G2) trigger. In this manner the least number of $K_{3 \pi}$ events were lost.

\subsubsection{Glass Cluster Finding Trigger Proceesor Efficiencies}

The GCF trigger processor efficiencies were determined from showers in the glass from $K_{2 \pi}$ triggers( see Section 4.2 ) which required a simple vee topology decay with at least one hit in each bank of each hodoscope array and the MLU trigger processor ' $K$ '. A program simulated the GCF trigger processor. When the simulation program calculated two or more clusters (the requirement for the $\mathrm{K}_{3 \pi}$ trigger), $86 \%$ of the time the GCF trigger processor also found two or more. The other $14 \%$ of the time it found only one cluster. These cases usually corresponded to events where 
$\frac{7}{0}$
5
0
0
$\dot{7}$
-1
7
0
0
5
3
0
0
0
0
0
0
0

Note: 1 all diodes are IN914

2 2Kohm-20 turn pot: set 687 threshold clockwise for lower threshold voltage

3 bias resistors are $1 \mathrm{Kohm}$

4 series output resistors are $10 \mathrm{ohm}$

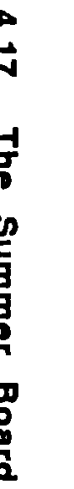

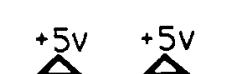

$+5 v+5 v$

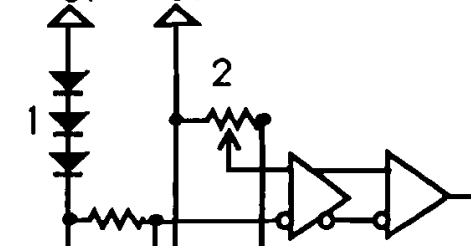

千 $\uparrow$

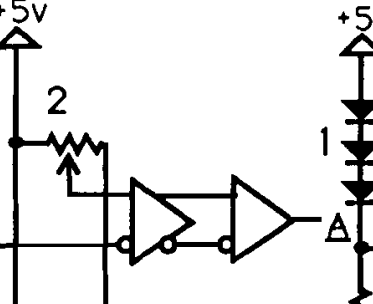

年 $\stackrel{+5 \mathrm{~V}}{\mathrm{~A}}$
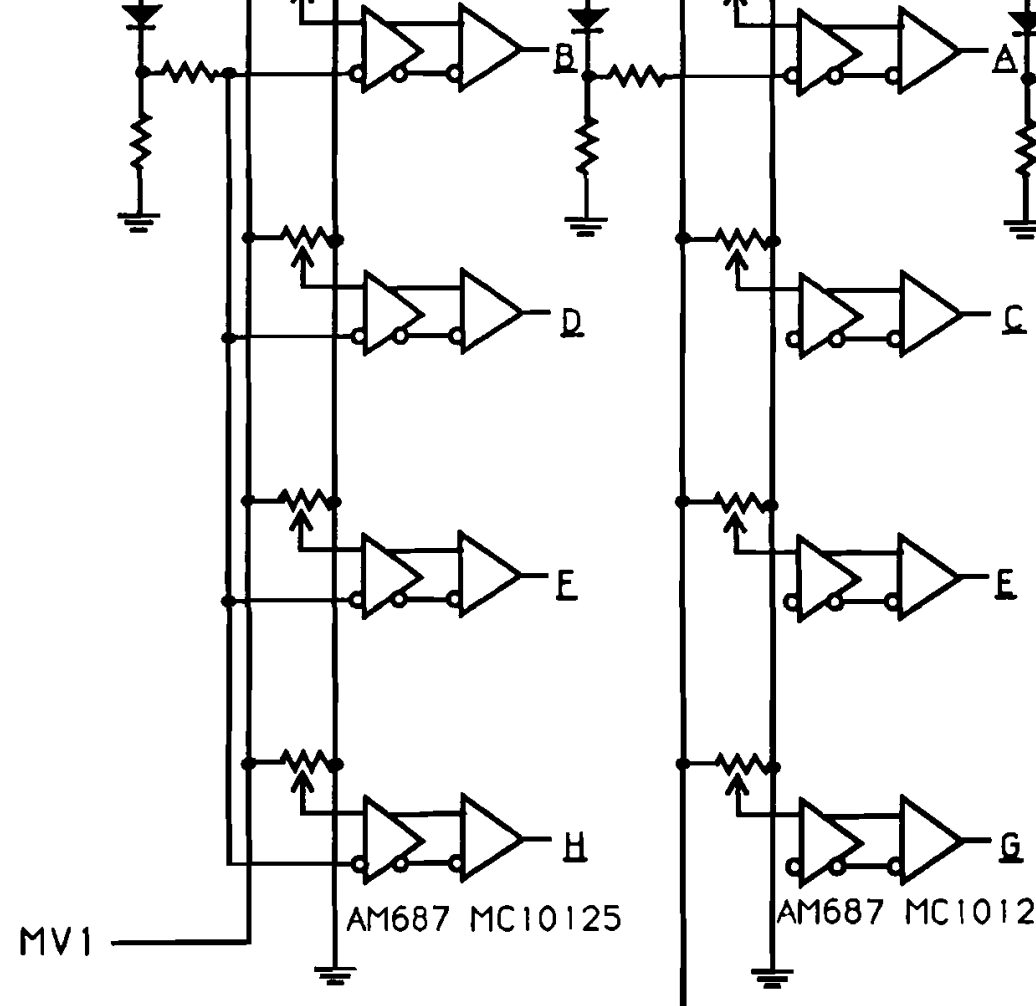

MV2

MV3

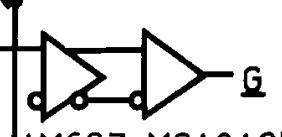

$\stackrel{1}{=}$

$\underbrace{2}_{\substack{A M 687 \\ \frac{1}{2}}}$

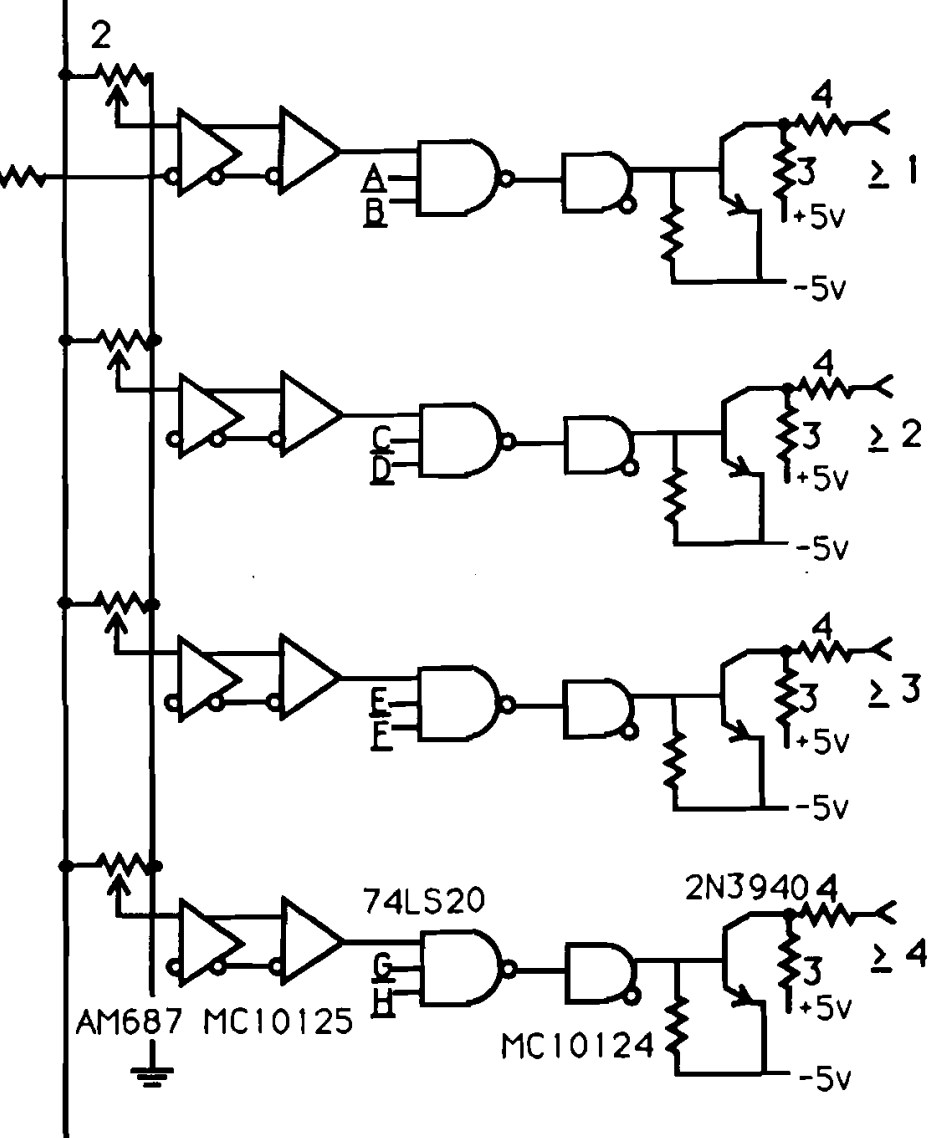


the second shower was 8 or more nsec out of time with the first shower and thus the signals probably did not sum correctly in the Summer Board to get two showers. The simulation program allowed signals to be 12 ns out of time. $95 \%$ of the one cluster simulation program events were correctly identified by the GCF trigger processor with a little over $1 \%$ of them being incorrectly identified as two clusters.

$I$ also looked at the GCF trigger processor efficiency in $K$ - G1 triggers which were $K_{2 \pi}$ triggers that required the GCF to find at least one cluster in the glass. I looked at events where the simulation program calculated two or more clusters. This was a stricter test of the GCF trigger processor as G1, one neutral cluster, was already required. On the average $78 \%$ of the time the GCF trigger processor calculated a G2 or two clusters or more when it should have.

Making a stricter timing requirement of the lead glass signals greatly increased the GCF trigger processor efficiencies. Using $K$ triggers and requiring the lead glass signals be within $8 \mathrm{~ns}$ of their average value, the GCF trigger processor efficiency was found to be $97 \%$ for finding two neutral clusters when it should have. Thus the efficiency of the GCF triggger processor is mostly dependent on the timing of the incoming glass signals. 


\section{Chapter Five}

\section{Data Acquisition}

\subsection{General}

At the rate of $5 \times 10^{11}$ protons on target approximately 3600 events per spill were put on tape. Of these events $68 \%$ were $\mathrm{K}^{\circ} \rightarrow 3 \pi$ triggers, $18 \%$ were $\mathrm{K}^{\circ} \rightarrow 2 \pi$ triggers and the rest were various triggers used for obtaining efficiencies and measuring other particle decays. Typically we ran with an $80 \%$ live time. It took about 100 spills at $5 \times 10^{11}$ to fill a tape with $250,000 \mathrm{~K}_{3 \pi}$ triggers corresponding to 370,000 total triggers. Each spill cycle was one minute long with a 20 second spill and a 40 second recovery. With $5 \times 10^{11}$ protons per spill, we could fill a tape in $1 \mathrm{hr}$ and 40 minutes. The front chambers' noise rates at this intensity were $12.9 \times 10^{6}$ for a 20 second spill or $0.65 \mathrm{MHz}$.

Since the same number of $\mathrm{K}_{3 \pi}$ events was desired for the upstream and the downstream target, most of the protons needed to be on the upstream target. This was because the solid angle of the collimator hole was much smaller for the upstream than the downstream target. The septum in PC1, shown in Figure 2.1, was therefore adjusted to put $93 \%$ of the beam on the upstream target and $7 \%$ on the downstream target. Any beam split more asymmetric than this was difficult to monitor on the SWIC's since so little beam was on the downstream target. With the 93/7 beam split we obtained 2 times as many reconstructed $\mathrm{K}_{3 \pi}$ 's from the downstream target as from the upstream target. We monitored this split by recording the total number of $\mathrm{K}_{3 \pi}$ triggers/proton where the number of protons in both beams was measured by a secondary emmision monitor (SEM). A small change in the split would drastically change this value because more $\mathrm{K}_{3 \pi}$ triggers came from the downstream target.

Table 5.1 shows the eight different running conditions used to measure any acceptance differences between the two beams. M1 and M2, M3 changed polarity periodically and the targets were moved from beam to beam. The positions of each target were adjusted electronically. The ratio of the number of protons on the east 
versus the west target is shown as $93 / 7$ or $7 / 93$ where 93 always corresponds to $93 \%$ of the proton beam on the upstream target.

\begin{tabular}{|c|c|c|c|c|c|}
\hline & & & BEAM RATIC & UPSTREAM & DOWNSTREAM \\
\hline BUN & PCBANA(M1) & PC4AN1.2 (M2.M3) & East/West & IARGEI & IARGET \\
\hline 1 & +3600 amps & +2500 amps & $93 / 7$ & east & west \\
\hline 2 & +3600 amps & -2500 amps & $93 / 7$ & east & west \\
\hline 3 & -3600 amps & -2500 amps & $93 / 7$ & east & west \\
\hline 4 & -3600 amps & +2500 amps & $93 / 7$ & east & west \\
\hline 5 & -3600 amps & +2500 amps & $7 / 93$ & west & east \\
\hline 6 & -3600 amps & -2500 amps & $7 / 93$ & west & east \\
\hline 7 & +3600 amps & -2500 amps & $7 / 93$ & west & east \\
\hline 8 & +3600 amps & +2500 amps & $7 / 93$ & west & east \\
\hline
\end{tabular}

Table 5.1 Elght Different Run Types

\subsection{Apparatus Alignment}

The A, B and $\mathrm{Pi}$ hodoscopes, the lead glass array and all the chambers were optically surveyed and leveled when they were installed. The center vertical wire of each chamber and the center of the three hodoscope arrays were aligned with the beam center line as defined in 2.4. This was accomplished by dropping a plumb bob to the floor where the beam center line was marked.

A "straight-through" run was used to more accurately align the MWPC's. All three magnets M1, M2 and M3 were turned off and a low intensity proton beam of $10^{6}$ protons/spill was sent through the west hole of the collimator. The center of the illumination in each chamber then defined the origin of the beam coordinate system. During data taking, chambers were occasionally removed in order to replace broken wires and their positions shifted slightly $\sim 1 \mathrm{~mm}$. Using the geometrical fit to the charged tracks of the data determined their new locations. This procedure is described in Section 6.2.1.

\subsection{The Readout Procedure}

Chamber and counter signals were arranged to make a logical decision which defined a trigger. Figure 4.3 showed the full trigger schematic. Several triggers were then OR'ed together and the output defined a good event. Thus if any one trigger 
were satisfied a "good event" signal would be generated. The good event signal caused all the chamber wire hit information, hodoscope information and lead glass array information to be read out and put on 6250 bpi magnetic tape. For each good event the data readout contained:

(1) MWPC wire hits

(2) ADC and TDC information for all lead glass block hits

(3) latched values for all the scintillators and triggers

When a good event (GE) gate occurred 'busy' signals were sent out to the various apparatus electronics to inhibit further triggers until the event was read out. A PDP-11/45 computer controlled our readout procedure. Once a 'GE' gate was produced a interrupt signal was sent to the PDP-11 causing the computer to start executing a CAMAC readout cycle as controlled by the on-line data acquisition software. An 'enable' signal was sent to the MWPC's to start the chamber readout and a signal was sent to both the ADC's and the TDC's to start the readout of the glass information. The on-line computer program was RT-MULTI ${ }^{(38)}$, a Fermilab system standard. Between spills an interrupt signal was sent to the PDP-11 causing the readout of various scalers and the $A D C$ pedestals.

All triggers except the $K_{3 \pi}$ trigger were electronically divided (prescaled) by various factors ranging from $2^{6}$ to $2^{15}$. The factors were selected to yield a statistically significant sample of triggers while minimizing experiment dead time.

\subsubsection{MWPC's}

The signal from each wire was amplified and each signal above threshold initiated a 940 ns long 'one-shot' signal. These one-shot signals were then differentiated. The leading edge signal defined the 'fast-OR' signal used in forming the triggers such as the F. C5 trigger or the CMR trigger. The trailing edge signal delayed the hit information until a trigger could be formed. This one-shot created a 'dead time' in which a wire was unable to respond to new inputs. This dead time defined a wire inefficiency we wished to keep to a minimum. The 940 ns delay was defined by the time it took for the signals to be registered, travel to the control room about 120 feet away ( $400 \mathrm{nsec})$, be processed by the trigger ( 140 nsec) and returned by cable to the MWPC's. Keeping the overall single wire hit rates in the chambers less than $0.6 \mathrm{MHz}$ kept the individual wire inefficiency small. In the 


\section{7}

central regions of the chambers the efficiency was $1-2 \%$ less than that near the edges of the chambers where the rates were lower.

The chamber enable signal generated by a good event trigger was $150 \mathrm{~ns}$ long. Only amplifier cards with signals in coincidence with this enable were latched at the chamber. Under computer control, the CAMAC interface typically read out one latched amplifier card every $2 \mu s$ (one CAMAC cycle). Only hit wire addresses were read out and the information was read out serially as a 16-bit address word.

\subsubsection{ADC's and TDC's}

The lead glass information consisted of the Lecroy 2280 analog to digital converter signals (ADC's) and the Lecroy 4291's time to digital converter signals (TDC's). The ADC's provided pulse height information for each lead glass block and the TDC's provided the time of arrival of that signal. Two sets of TDC's were used and read out. Each block had a TDC which recorded signals above a threshold of $10 \mathrm{mv}$ $(\sim 1 \mathrm{GeV})$ The other set of TDC's recorded signals from blocks used in the GCF trigger with a $25 \mathrm{mv}(\sim 2 \mathrm{GeV})$ threshold. The timing of the high threshold set of TDC signals was important since the GCF trigger processor used those lead glass block pulses. We closely monitored these TDC signals and corrected any shifts in their average times by adding or removing cable from their signal lines. The cable lengths were adjusted after each lead glass calibration run since some lead glass blocks were replaced and many phototube voltages were adjusted at that time. Variations in cable lengths typically corresponded to a \pm 2 nsec variation.

A 200 ns wide gate was sent to the Lecroy 2280 ADC's which digitized signals in an integrated charge mode with 12-bit resolution. The ADC controller subtracted the pedestal (residual count) from each channel. The TDC's digitized the time from when they first received a signal to the time when they recieved the 'common stop' signal provided by a good event gate. Their output was 10 bits with 1 ns per bit resolution.

\subsubsection{Latches and Scalers}

The latches contained delayed signals from all the scintillator elements and triggers which were in coincidence with a good event gate and thus associated with the event being read out. These 16-bit latch words were used to identifiy which triggers were satisfied by the event and to check trigger processor and hodoscope efficiencies.

Scalers counted the total number of input signals for various devices in the 
experiment such as chamber fast-OR pulses, scintillators (V1, DK) and prescaled triggers. They were used to monitor trigger and chamber rates during a spill in order to determine the dead-time of the experiment and to check prescaler values for the prescaled triggers. Both gated and ungated scalers were recorded to measure experimental dead time.

\subsubsection{Between Spill Readout}

Between each spill the ADC pedestals and all scalers were read out. Signals were also sent to the TDC's and the ADC's to re-establish their zero points.

The ADC pedestals values were sent back to the ADC processor so that they could be subtracted from all the ADC channels for all the events in the next spill. Only non-zero $A D C$ channels were read out thereby reducing the length of the event record. The signal sent by the computer to the ADC's reset the reference voltage level which set the scale for the ADC digitization. This assured that the ADC calibration was maintained. SImilarly the timing signal sent to the TDC's 'zerotrimmed' each TDC channel to maintain their calibration.

The scalers read out between spills counted spill rates for the various triggers and counters. Once read out they were then cleared in preparation for the next spill.

\subsection{Recording Event Data}

Information was transferred to the PDP-11 computer via a CAMAC-PDP interface. Each event record was typically 50 16-bit words. These were combined into 1920-word, 160 bits/word, buffers in computer memory and then written on to a 6250 bpi 9-track magnetic tape. The readout could write up to 1000 events/sec, the limiting factor being the CAMAC readout driver.

The on-line computer program used for the event readout also had histogramming capabilities. All chamber wires, ADC's, TDC's, event dumps, trigger processor latches and hodoscoope latches could be displayed as the data was being recorded. This histogramming capability was invaluable in monitoring electronics performance.

\subsection{Electron Positron Data}

Electron/positron data was needed to correctly calibrate the lead glass blocks while we took data. It was also very useful for determining the characteristics of electromagnetic showers in our detector. The photon shower Monte Carlo depended on 
the $e^{+} e^{-}$data in order to simulate the photon showers in $\mathrm{K} \rightarrow 3 \pi$ decay. Several sets of $\mathrm{e}^{+} \mathrm{e}^{-}$data were taken during the data acquisition period.

Because the lead glass array was used in the trigger, the high voltage for each phototube was adjusted to give each block approximately the same energy response. Electron/positron runs were taken to determine the condition and optimum voltages of the lead glass blocks. It was discovered that the lead glass blocks in the central region of the array became yellow from the neutral beam. These blocks needed to be replaced every few weeks. As time went on blocks further from the hole in the array needed to be replaced also. A mercury lamp was used to clear the badly damaged blocks and some mildly damaged blocks were moved to the edge of the array to be replaced later.

To take $\mathrm{e}^{+} \mathrm{e}^{-}$data tapes a $0.6 \mathrm{~cm}$ thick piece of lead was placed in the beampipe bellows slightly upstream of the V1 scintillator. Photons in the neutral beam then converted to $\mathrm{e}^{+} \mathrm{e}^{-}$pairs in the lead. For these runs, the trigger was changed slightly to require $V 1$ in coincidence instead of veto:

$$
V 1 \cdot D K \cdot\left(\left(H A_{L} \cdot H A_{R}\right) \cdot\left(H B_{L} \cdot H B_{R}\right)\right) \cdot M L U \cdot C M R \cdot G_{1}
$$

The MLU trigger processor was reprogrammed for "vees". This trigger was satisfied if there were at least one hit in each side of the $A$ and $B$ hodoscope arrays. This together with the requirement of the CMR trigger processor that identified events with symmetric slopes, resulted in over half of the triggers being reconstructable $\mathrm{e}^{+} \mathrm{e}^{-}$pairs. The $\mathrm{Pi}$ hodoscope signals used for vetoing charged showers in the lead glass array were removed from the GCF trigger processor veto boards for these runs since charged showers were desired in the lead glass array. The GCF trigger processor then was required to find at least one cluster in the lead glass array (G1). For these runs the intensity of the beam was reduced to $5 \times 10^{10}$ protons per spill.

A small dipole magnet, PC4S, was put in the beamline downstream of V1. This magnet seperated the $\mathrm{e}^{+} \mathrm{e}^{-}$beam vertically. The analyzing magnets $\mathrm{M} 2$ and $\mathrm{M} 3$ were used to move the beam horizontally. By using these magnets as well as displacing the glass array horizontaly we were able to illuminate the whole lead glass array with $e^{+} e^{-}$showers. 
The tapes taken under these conditions were immediately analyzed to determine the response for each block in the array. From this information it was determined which blocks should be replaced and which phototube voltages should be adjusted. 


\section{Chapter Six}

\section{Data Reconstruction}

\subsection{Introduction}

The events recorded on the data tapes were analyzed to identity neutral particle decays. When evidence for a decay was found, the analysis program attempted to reconstruct the decay vertices, particle momenta and positions from the chamber signals. These quantities were used to identify the event as a $\Lambda, K_{2 \pi}$ or $K_{3 \pi}$ candidate, depending upon the mass reconstructed from the charged tracks. Those events which were identified as $K_{3 \pi}$ candidates were further analyzed for evidence of photon showers in the lead glass array. The two photon shower combination that yielded the best $\pi^{0}$ mass was then used to reconstruct the $K_{3 \pi}$ mass and momentum.

In order to determine the momenta and positions of the charged particles, the positions of the MWPC's and the analysis magnets and the analysis magnets' magnetic fields were measured. The neutral beam and target positions were also necessary for the analysis. For the $\pi^{0}$ momentum, lead glass calibration constants and positions were needed.

\subsection{Determining Apparatus Positions}

All $\mathrm{z}$ positions were measured to $+/-2 \mathrm{~mm}$ with a measuring tape and a plumb bob which was dropped to the surveyed line on the floor of the beam center line. Once $x$ and $y$ positions were also determined as described in the Sections 6.2.1-6.2.6, Monte Carlo and data comparison plots of charged track and neutral track projections to various points in the spectrometer matched beautifully in width and structure. Figure 6.1 shows Monte Carlo and data comparison plots of the $x$ and $y$ of the charged tracks projected to the analysis magnet's downstream face. Figure 6.2 shows Monte Carlo and data comparison plots of the $x$ and $y$ of the photon showers at the lead glass array. The curves are drawn throuh the data points. The spikes in the plots of the photons projected to the lead glass array are due to the position resolution of the 
array and reflect the structure of the array.
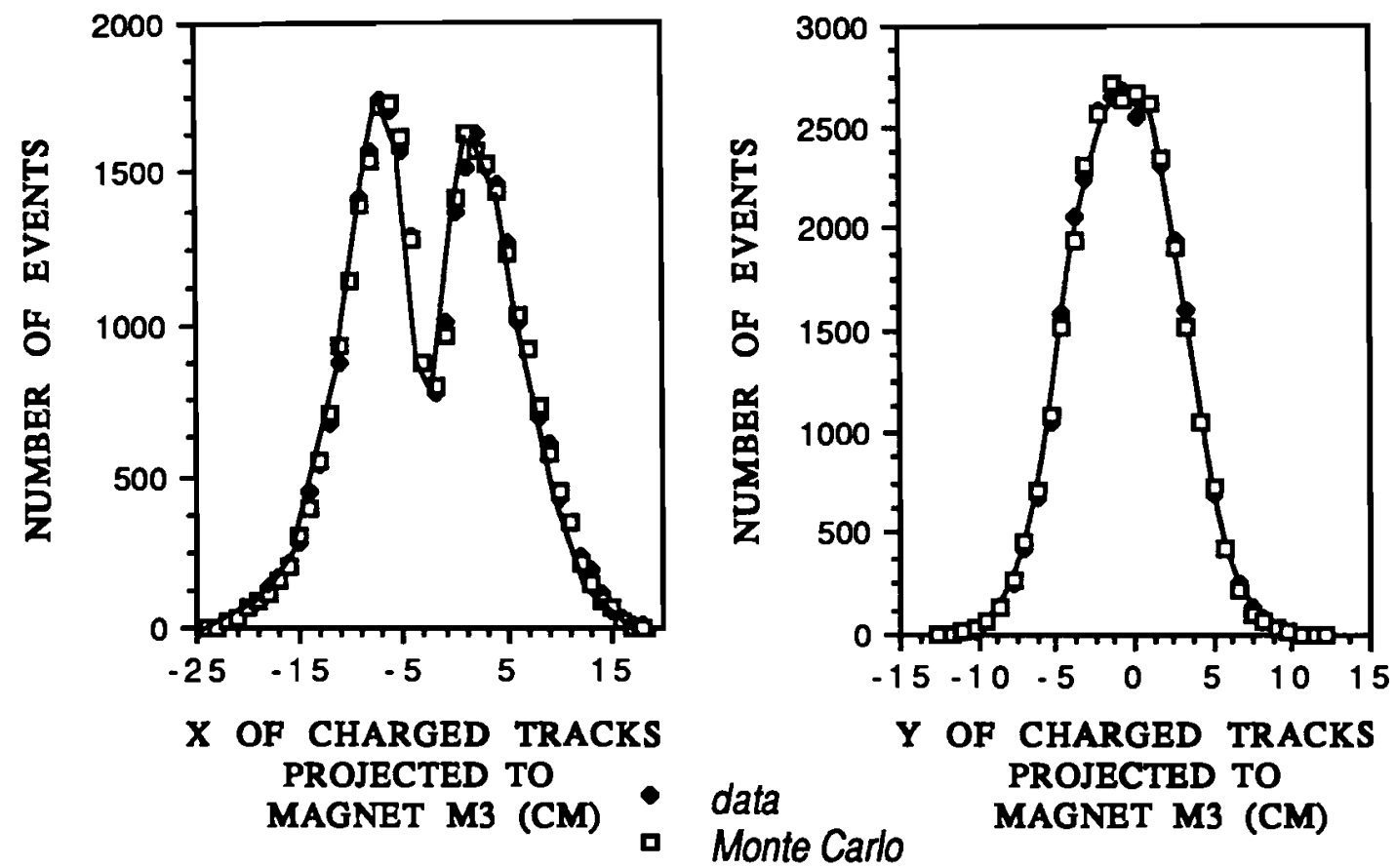

Flgure 6.1 $X$ and $Y$ of the Charged Tracks at the Analysis Magnets
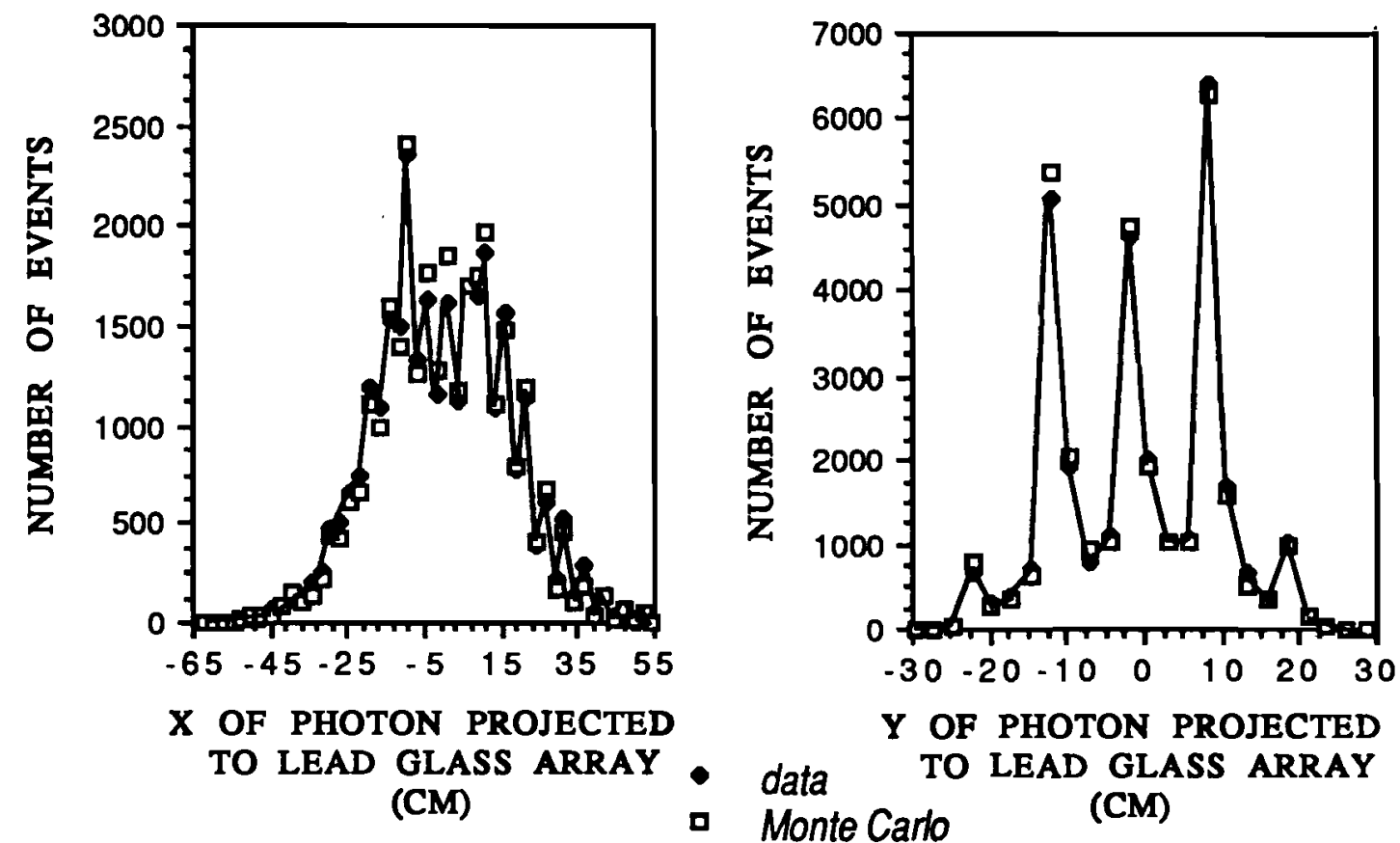

Figure 6.2 $X$ and $Y$ of the Photons at the Lead Glass 


\subsubsection{Chamber Positions}

It was important to obtain the best chamber positions for accurate kinematic reconstruction of the two charged tracks in the $\mathrm{K}_{3 \pi}$ event. Also in order to simulate the CMR trigger processor accurately the $\mathrm{x}$ positions of the wires in C4 and C5 were needed.

Because chamber planes were occasionally removed during the data taking, chamber $\mathrm{x}$ and $\mathrm{y}$ positions were determined tape by tape. The precision of the determination depended on the accuracy of the simple simulation of the analysis magnets' fields in the reconstruction program (discussed in Section 6.3).

The chamber plane positions were determined from a measurement of the distance from the actual chamber hit to the projected track postion determined by the reconstruction program. It is in essence a measurement of the chamber offset in $x$ and $y$. Adjusting the offsets to zero gave a new set of chamber positions. These new postions were then used to re-analyze the same data to determine a new set of offsets. This process continued until all offsets were $<0.5 \mathrm{~mm}$, approximately three iterations. The positions determined from this first tape were then used as the starting positions for subsequent tapes. This process continued for each tape in order. The only times when a chamber offset became large, $\sim 0.7 \mathrm{~mm}$, always corresponded to when a chamber was removed for repairs which happened only five times during the course of the experimment. Only the chamber actually removed and replaced needed adjustment.

Files were made of these chamber positions for the analysis and the Monte Carlo program. The front chambers' offsets were typically $<0.08 \mathrm{~mm}$. The back chambers, especially $\mathrm{C} 6$ since it was not necessarily required in the track fitting program, were sometimes $\sim 0.4 \mathrm{~mm}$ but usually $<0.2 \mathrm{~mm}$. Figure 6.3 shows the residual (offset) for chamber $\mathrm{C} 5$ in $\mathrm{x}$ and $\mathrm{y}$ for both Monte Carlo and data.

Chamber rotations about the $z$ axis were determined by looking for a $y$ offset dependence on the $\mathrm{x}$ position of the track or $\mathrm{a} x$ offset dependence on the $\mathrm{y}$ position of the track. The $\mathrm{x}$ offset versus the $\mathrm{y}$ position and the $\mathrm{y}$ offset versus the $\mathrm{x}$ position were fit for each chamber and the average taken to find the actual rotation angles. All were found to be less than 1 mrad. When they were used in the analysis program, they had no significant effect on either the chamber offsets or the chi-squared of the fit to the charged tracks determined by the program. For these reasons chamber rotations were taken to be zero in the reconstruction of the charged tracks. 


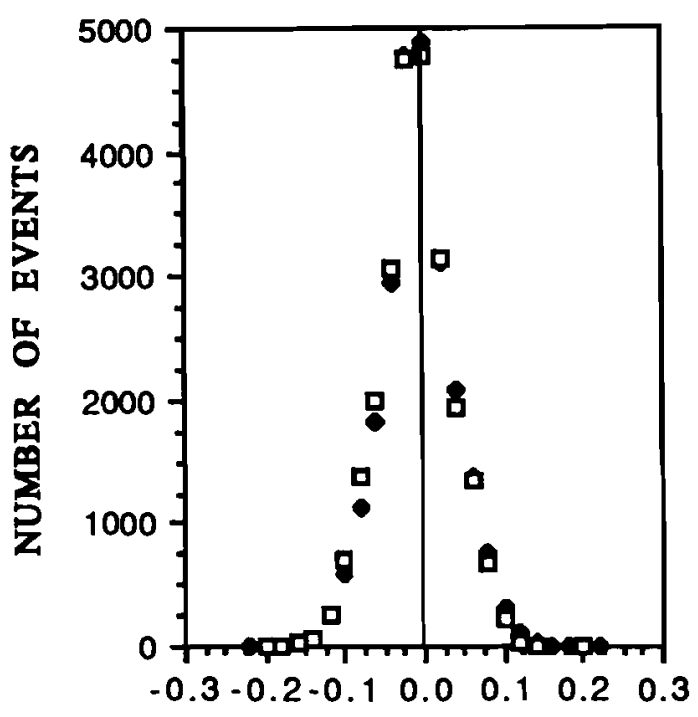

RESIDUAL C5X (CM)

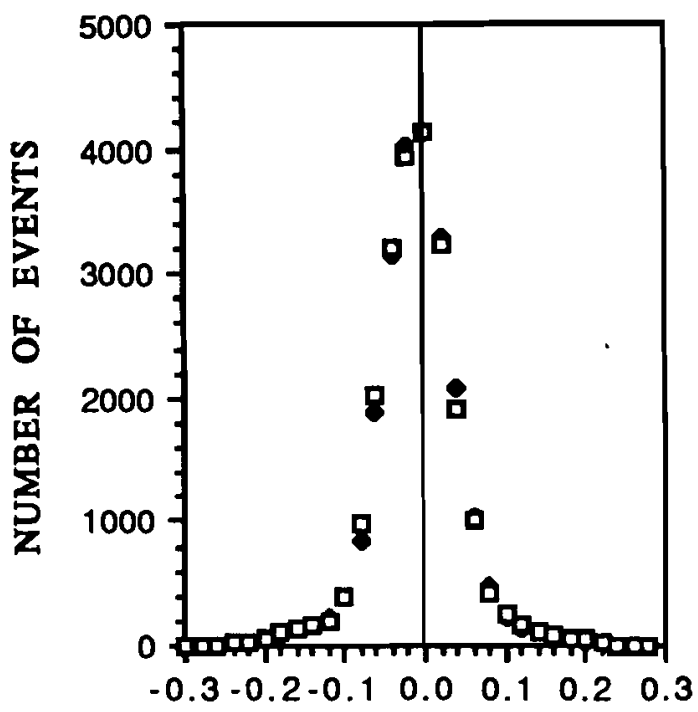

RESIDUAL C5Y (CM)

- data

- Monte Carlo

Figure 6.3 Chamber C5x and C5y Residual

\subsubsection{Beam and Target Positions}

Since the beam position at the target was used to remove kaons produced by the residual proton beam hitting the collimator, determining the beam position on the target was important. The positions of the two targets in each beamline were surveyed optically to $0.02 \mathrm{~mm}$ in $x$ and $y$ and $5 \mathrm{~cm}$ in $z$. The average position of the neutral kaon beam projected to the upstream target, the downstream target and the lead glass array and the angle of the neutral kaon beam entering the spectrometer were determined tape by tape from the data and were used in the Monte Carlo simualtion. On the average the beam fluctuated $0.6 \mathrm{~mm}$ in $\mathrm{x}$ and $\mathrm{y}$ on the downstream target and $1 \mathrm{~mm}$ in $\mathrm{x}$ and $\mathrm{y}$ on the upstream target. The angle of the neutral kaon beam with respect to the target-vetex axis and the position of the neutral kaon beam at the upstream target and the lead glass array are shown in Figures 6.4-6.6 for the downstream east $\mathrm{K}_{3 \pi}$ sample. Both Monte Carlo and data are shown on linear and logarithmic scales. 

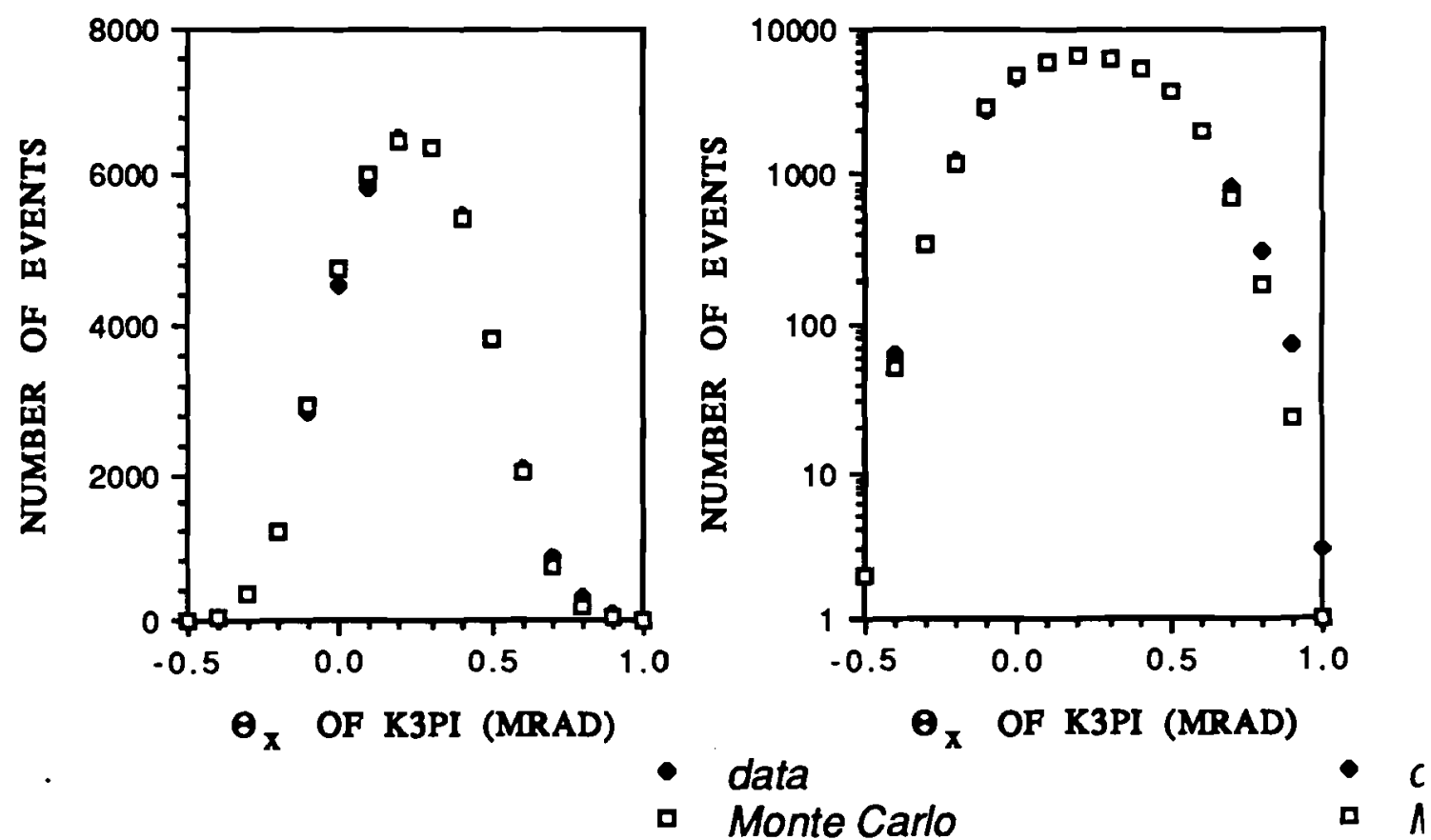

Figure 6.4a $K_{3 \pi}$ Downstream East Beam Angle in $X$
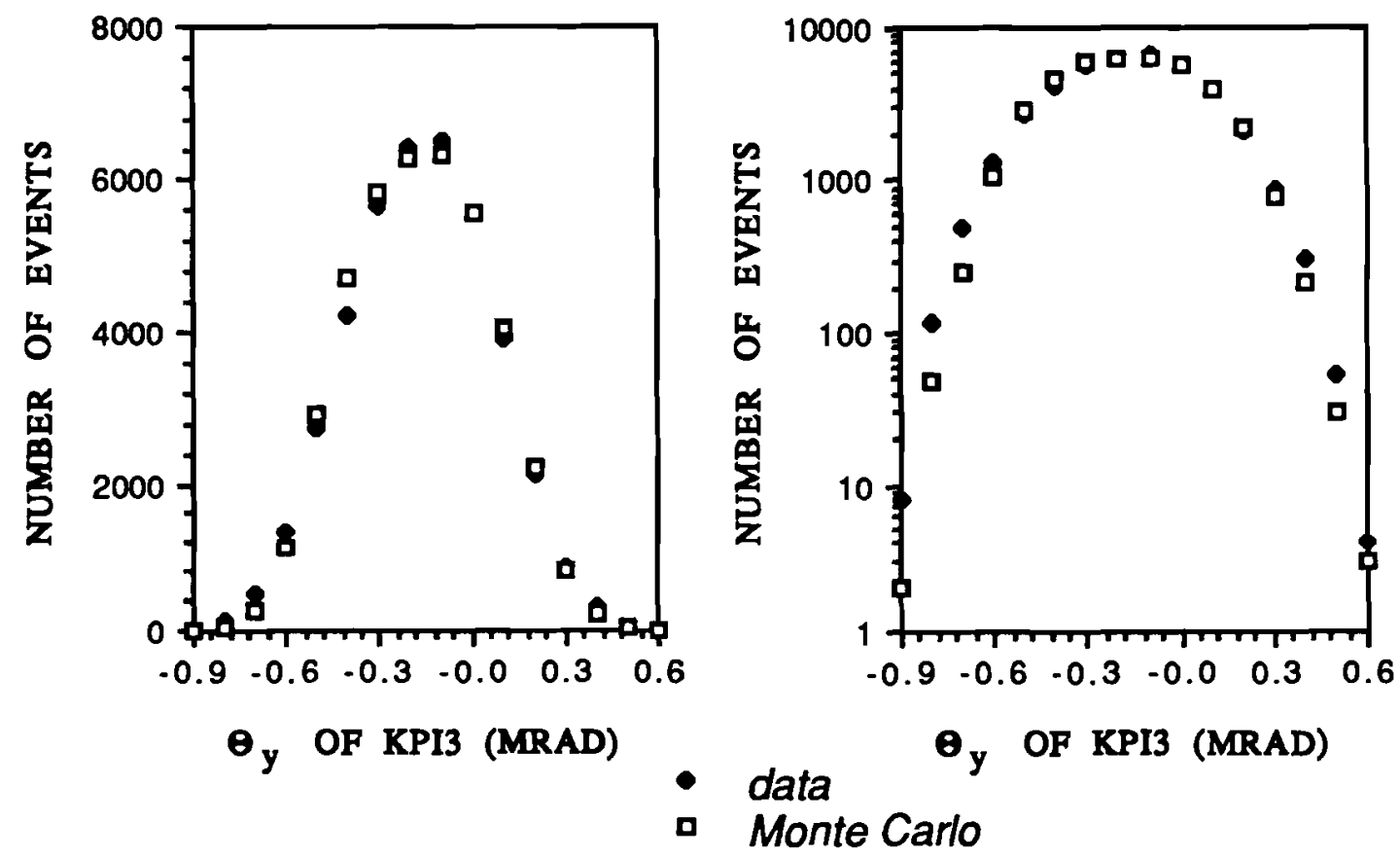

Figure 6.4b $K_{3 \pi}$ Downstream East Beam Angle in $Y$ 


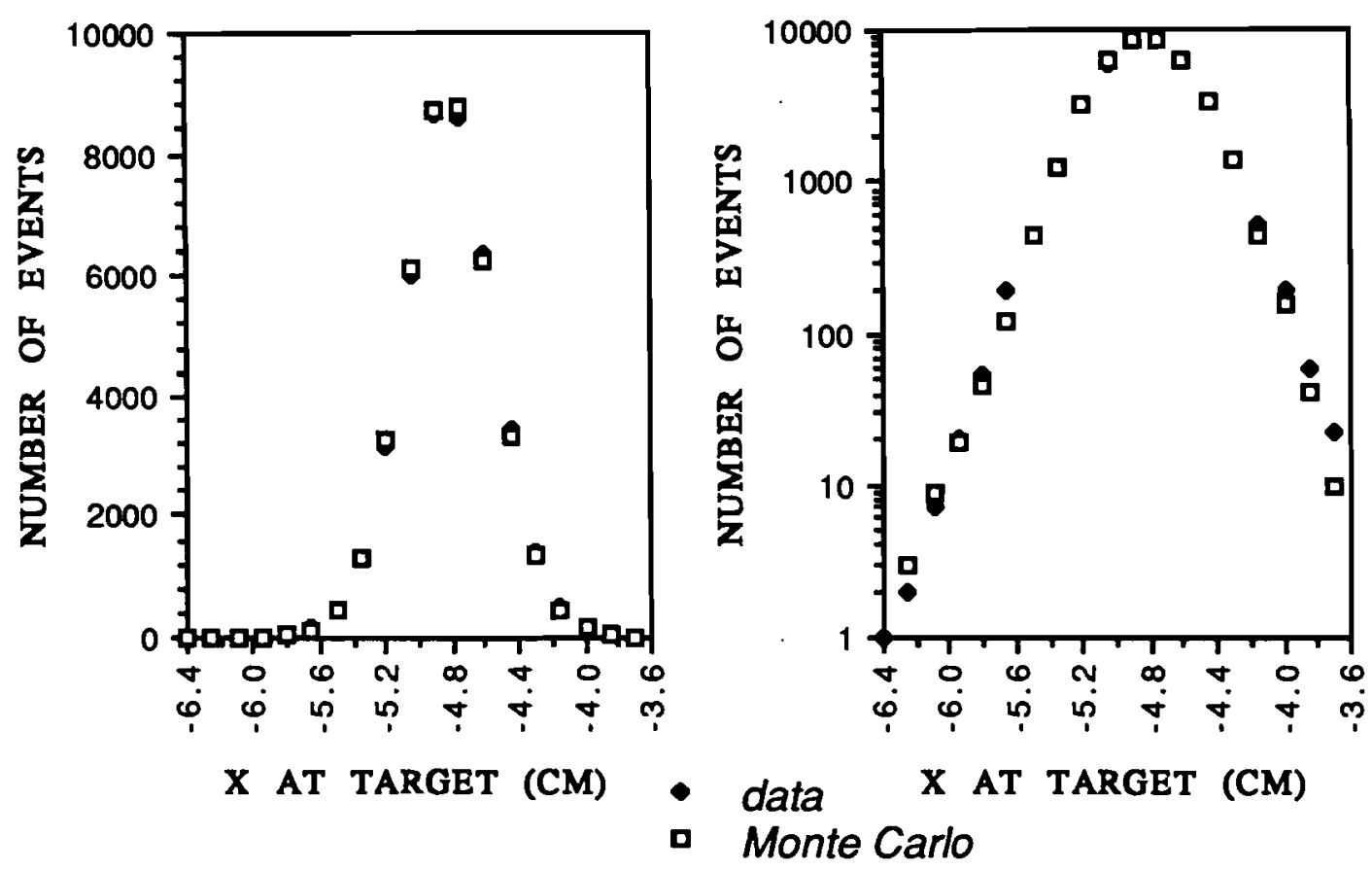

Figure 6.5a $K_{3 \pi}$ Downstream East Beam Projected to Upstream Target In $X$

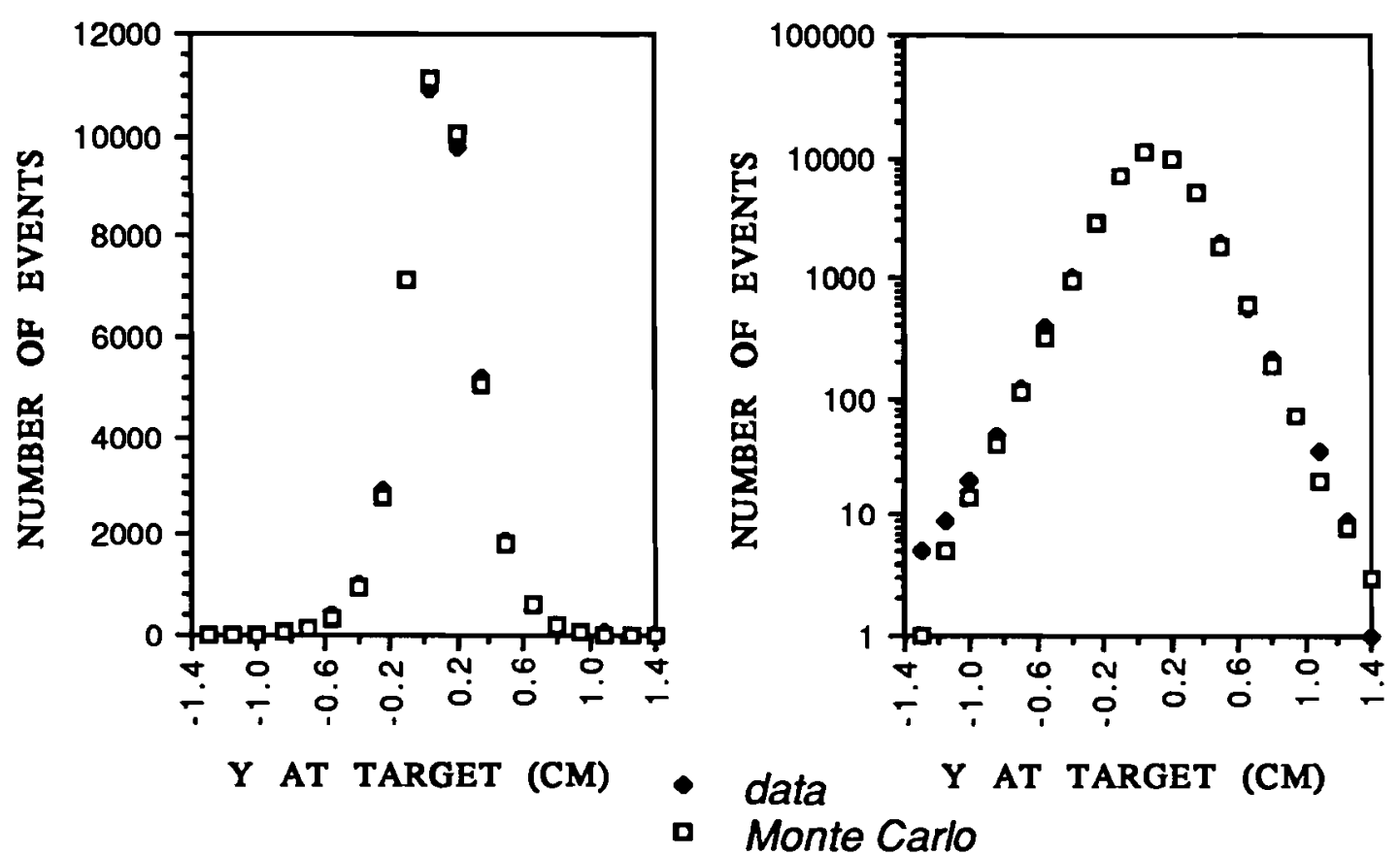

Figure 6.5b $K_{3 \pi}$ Downstream East Beam Projected to Upstream Target in $Y$ 

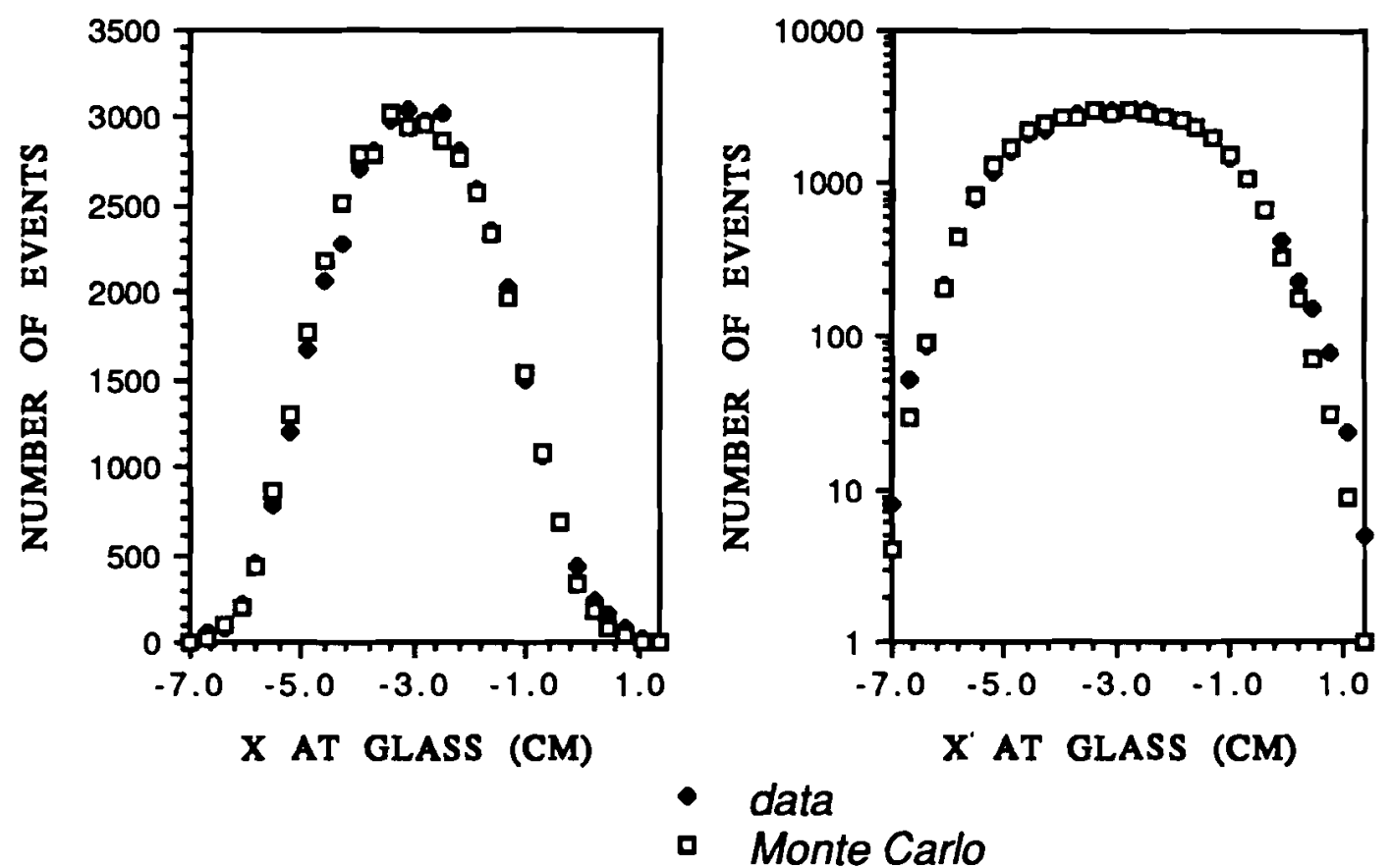

Flgure 6.6a $K_{3 \pi}$ Downstream East Beam Projected to Lead Glass Array in $X$

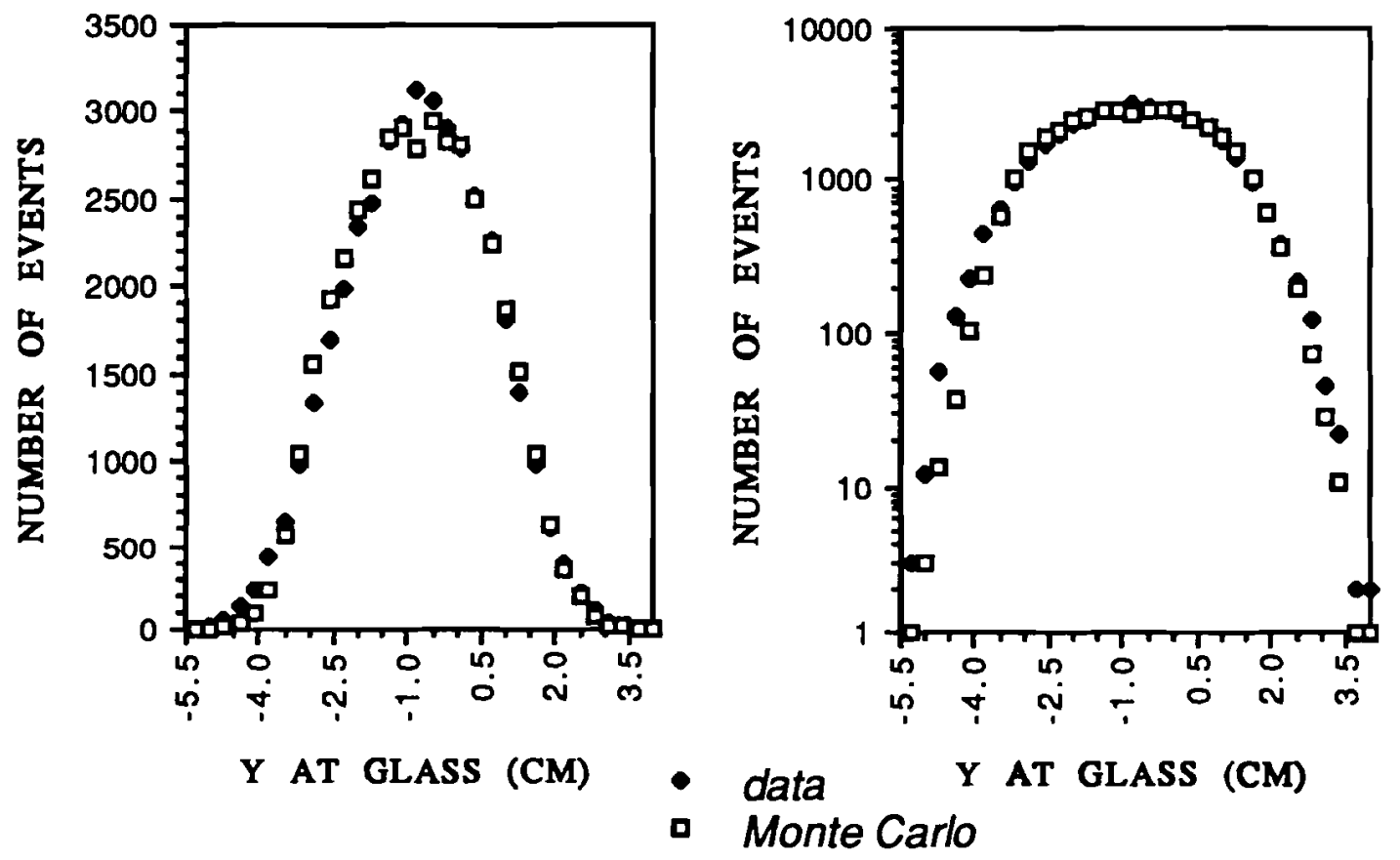

FIgure 6.6b $K_{3} \pi$ Downstream East Beam Projected to Lead Glass Array in $Y$ 


\subsubsection{V1 and DK Counter Positions}

The V1 and DK counter positions were determined by the particles produced at the counters as shown in the reconstructed $\mathrm{z}$ vertex distribution of $\mathrm{K}_{3 \pi}$ candidates from all triggers in Figure 6.7. The only requirement for these $\mathrm{K}_{3 \pi}$ candidates was that the reconstructed charged track mass not correspond to a lambda or a kaon mass.

Taking a weighted average of the $\mathrm{z}$ vertex for these particles I verified that the $\mathrm{z}$ of the $\mathrm{V} 1$ counter was $0.8 \mathrm{~m}$ and the $\mathrm{z}$ of the DK counter was $19.5 \mathrm{~m}$, in agreement with the optical survey. The $z$ vertex distribution of the final $K_{3 \pi}$ sample is given in Figure 6.8. The data events upstream of $V_{1}$ in the final $K_{3 \pi}$ could be accounted for by a $40 \%$ inefficiency in the counter $V 1$. The $K_{3 \pi}$ events downstream of DK could be completely accounted for by a accidental rate of $2 \%$ in the DK counter. The events upstream of $\mathrm{V} 1$ and downstream of DK were enough outside the decay region used in my analysis, 2-19 meters, that these results did not effect the analysis even when the resolution of the spectrometer was considered. Figure 6.8 shows the $z$ vertex of the $\mathrm{K}_{3 \pi}$ decay for data and Monte Carlo where the Monte Carlo is shown with and without the $V_{1}$ inefficiency and DK noise. The inefficiency for V1 used in the Monte Carlo in Figure 6.8 is $12 \%$ versus the $40 \%$ which agreed beautifully with the data because $12 \%$ was the highest inefficiency I could justify.

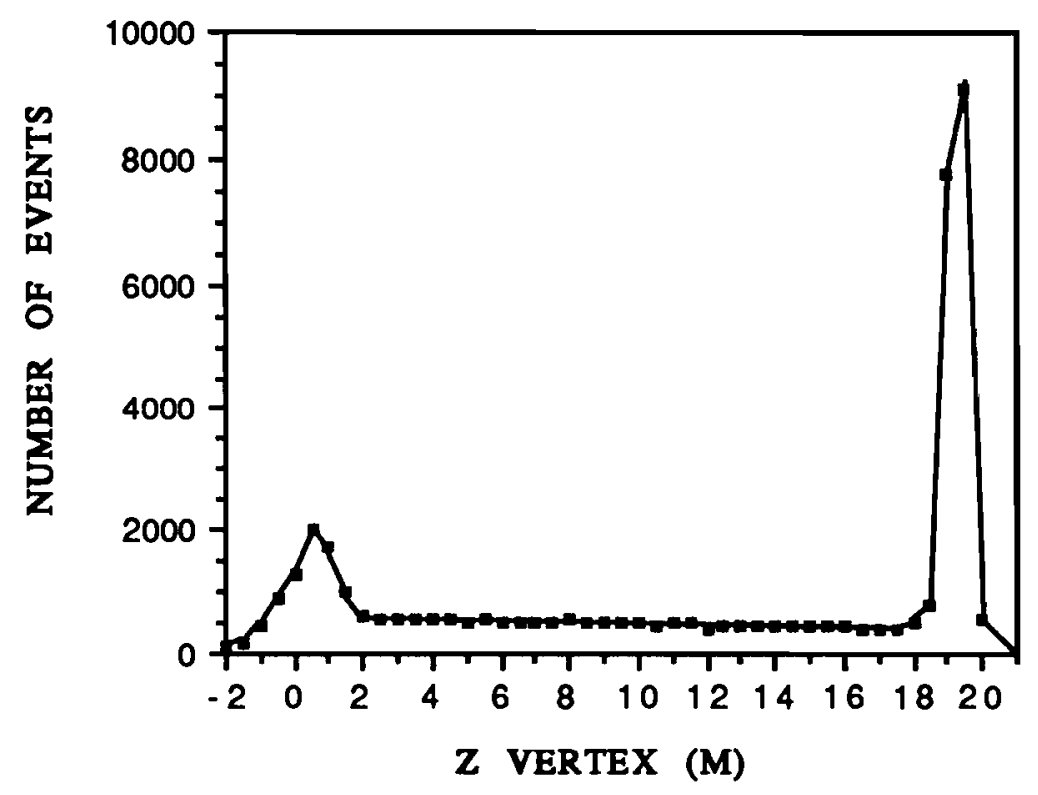

Figure $6.7 \mathrm{Z}$ Vertex Distribution, all Trlggers: PrellmInary $K_{3 \pi}$ Candldates 


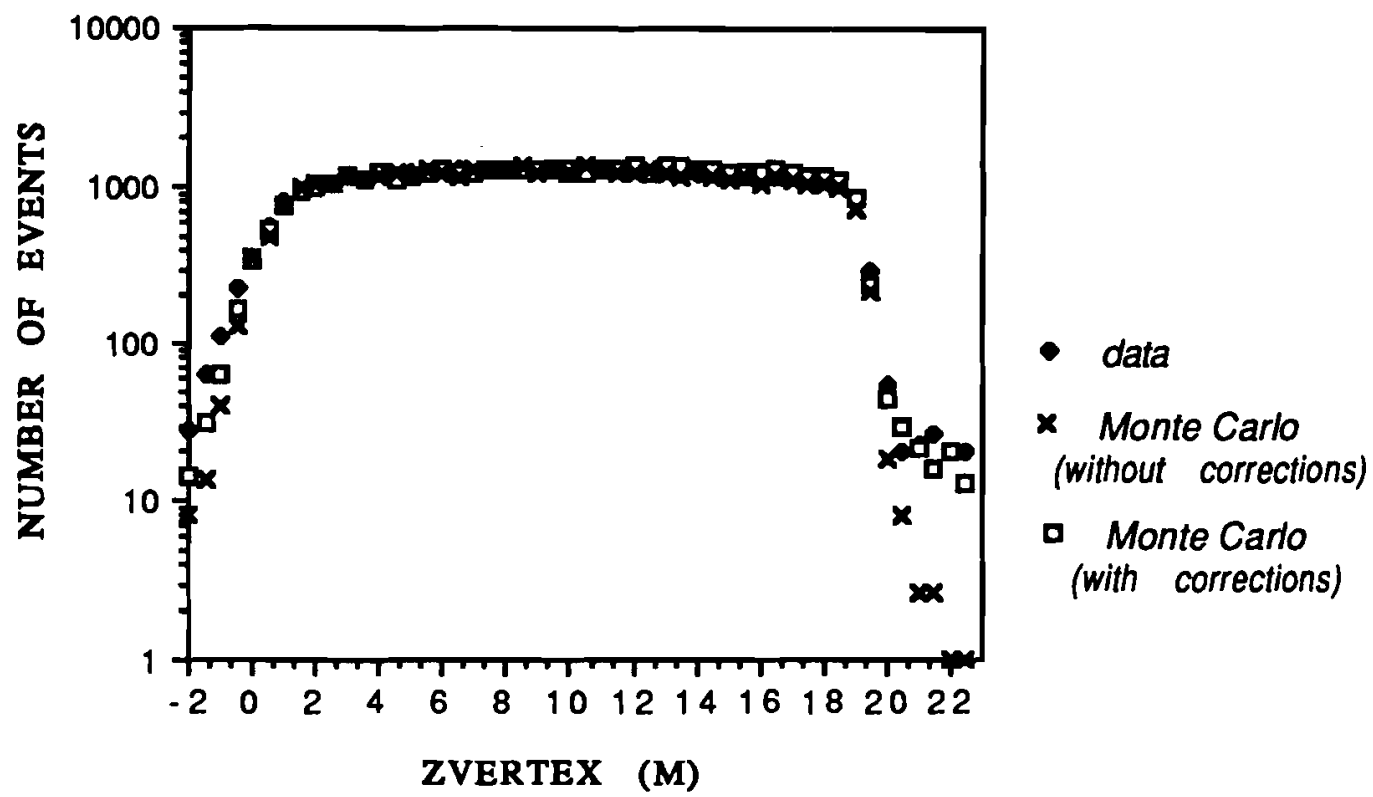

Figure 6.8 Full $Z$ Vertex Dlstribution for Final $K_{3 \pi}$ Sample

\subsubsection{Hodoscope Positions}

The MLU trigger processor used the $\mathrm{A}$ and $\mathrm{B}$ hodoscopes to measure the ratio of the slopes of the charged tracks and remove events with slope ratios less than 0.3 and greater than 3.0. In the analysis program a subroutine was used to simulate the MLU trigger processor and events that did not pass this software MLU cut were removed from the $K_{3 \pi}$ data sample. This made an accurate determination of the $A$ and $B$ hodoscopes positions important.

Each of the $A$ and $B$ hodoscope elements overlapped its neighbors by anywhere from 3 to $9 \mathrm{~mm}$. A sample of data where two neighboring elements were hit was used to determine the hodoscope positions to within $1 \mathrm{~mm}$.

The $\mathrm{Pi}$ hodoscopes were used in the GCF trigger processor simulation and thus their positions were also needed. They overlapped over a $10 \mathrm{~cm}$ square region in $\mathrm{x}$ and $y$ making the overlap determination of their positions accurate to $0.5 \mathrm{~cm}$.

\subsubsection{Analysis Magnets M2 and M3}

The analysis magnets M2 and M3 were optically surveyed into position. I determined the precise positions from the projection of the charged tracks to the 
magnet face. Any offset in the magnet aperature in $x$ or $y$ in the Monte Carlo lead to an offset in the projection of the charged tracks to the magnets. A Monte Carlo with the magnet aperature centered at zero in $\mathrm{x}$ and $\mathrm{y}$ best matched the data. The projection of the charged tracks to magnet M3 was shown in Figure 6.1.

\subsubsection{Glass Positions}

The lead glass block positions were needed since they were used to obtain photon energies and positions. They were also used in the GCF trigger processor simulation routine in the Monte Carlo. Their positions were optically surveyed with respect to the center of the lead glass array at the start of the data acquisition period. The positions of the blocks with respect to one another were measured with a measuring tape after each restacking of the lead glass array to replace radiation damaged blocks. The array center position was determined from the $\mathrm{e}^{+} \mathrm{e}^{-}$data taken at the start of each data set after restacking.

Using the chamber hit information, the $\mathrm{e}^{+} \mathrm{e}^{-}$tracks were reconstructed to determine the actual $(x, y)$ track coordinates of the $\mathrm{e}^{+}$or $\mathrm{e}^{-}$at the lead glass array. This was compared to a crude glass position determined by summing the first moments of each hit block in a cluster as in equation 6.1 .

$$
x_{\text {glass }}=\sum_{i} \frac{E_{i} x_{i}}{E_{\text {total }}}
$$

$\mathrm{E}_{\text {total }}$ is the total energy fo the shower, $\mathrm{E}_{i}$ is the energy in the $i^{\text {th }}$ block, $\mathrm{x}_{i}$ is the $\mathrm{x}$ coordinate of the center of the block $i$ and $\mathrm{x}_{\text {glass }}$ is the crude $\mathrm{x}$ position determined from the glass block energies. $Y_{\text {glass }}$ was determined in the same manner. For this first iteration in determining the lead glass array center the optically surveyed position was used.

A comparison of the shower center in the lead glass array and the track position projected to the lead glass array, for the electron/positron data, defined a correction to the initial value of the lead glass array center position. The new lead glass array center position was used in the next iteration and so on until the process converged in three iterations. For each data set, (a data set being defined as a set of tapes taken over a period in which the lead glass was not altered in any way), an array center was determined using this process. 


\subsection{Magnetic Fields}

In order to determine the momentum of the charged tracks and thus the mass of the neutral parent particle accurately, the magnetic field of the analysis magnets needed to be known. The field in the two magnets was oriented in the $y$ direction causing the transverse momentum component $\mathrm{P}_{\mathrm{xz}}$ of the charged particles to be rotated about the $y$ axis when they passed through the magnets. This rotation is related to the difference between the $\mathrm{x}$ veiw slopes of the particle tracks upstream and downstream of the magnets by equation 6.2.

$$
\sin \phi=\mathbf{P}_{\mathrm{T}} / \mathbf{P}_{\mathbf{x z}}
$$

where $\phi$ is the relative angle between the $x$-veiw tracks upstream and downstream of the magnets and $P_{T}$ is the transverse momentum kick of the magnetic field as defined in equation 6.3.

$$
\mathrm{P}_{\mathrm{T}}=3 \times 10^{-4} \overrightarrow{\mathrm{B}} \cdot \overrightarrow{\mathrm{L}} \quad(\mathrm{GeV} / \mathrm{C})
$$

This equation assumes a uniform field of magnitude $B$ in kiloGauss for a length $\mathrm{L}$ in centimeters. For a uniform magnetic field of length $\mathrm{L}$ the situation is shown in Figure 6.9.

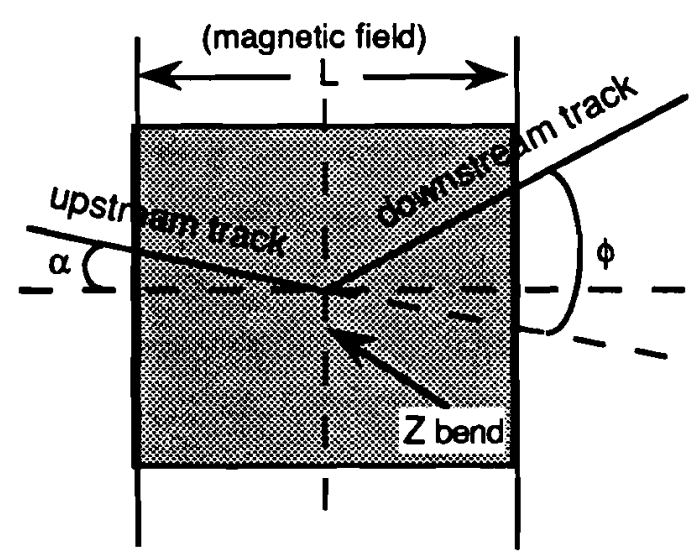

Flgure 6.9 The Efflectlve Bend Plane

For two magnets, as in E621, the expression for the position of the effective bend plane needed to be expanded to lowest order in $\phi$ and $\alpha$ as a function of the two magnets field lengths $\ell$ and $\ell$, the gap $s$ and the ratio $\lambda$ of the first magnet's $P_{T}$ to that of both magnets together. This expansion is given in equation 6.4. Figure 6.10 shows the two magnet setup of E621 and the parameters of interest. 


$$
\begin{aligned}
Z_{\text {bend }}= & 1 / 2\left[(1+s)(2-\lambda)+\left(\ell^{\prime}+s\right)(1-\lambda)-s\right] \\
& +1 / 2\left[(1+s) \lambda(3-2 \lambda)+\left(l^{\prime}+s\right)(1-\lambda)(1+2 \lambda)\right] \alpha \phi \\
& +1 / 2\left[(1+s) \lambda\left(2+\lambda^{2}\right)+\left(l^{\prime}+s\right)(1-\lambda)(1+\lambda)^{2}\right] \phi^{2}
\end{aligned}
$$

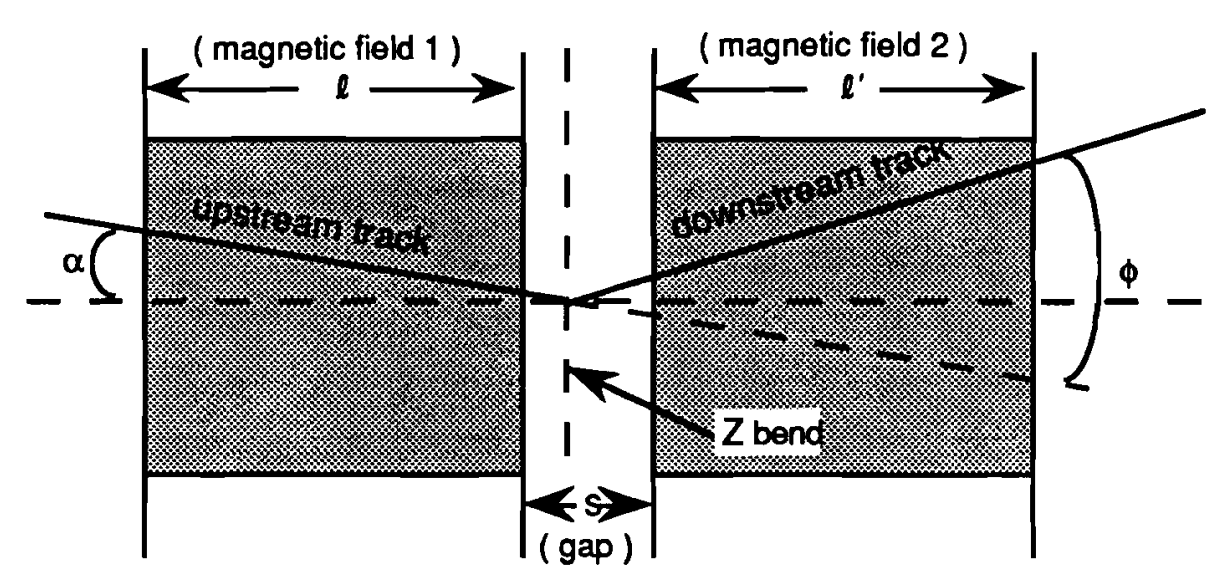

Flgure 6.10 The Effectlve Bend Plane for Two Magnets

$\mathrm{Z}_{\text {bend }}$ determined from equation 6.4 is relative to the upstream end of the first magnet's magnetic field. The analysis magnets' field parameters are given in Table 6.1. The values in Table 6.1 for $\ell, \ell^{\prime}$ and $s$ were determined from the previous experiments' (E619) magnetic field measurements for M2 and M3. In E619 the same magnets were used but they were run at a different current and their relative positions were different. Assuming the magnetic fields were symmetric in $\mathrm{z}$ about the individual magnets and that the current was proportional to the magnetic field strength, the magnetic field lengths $\ell$ and $\ell^{\prime}$ could be determined from the E619 measurements. The gap width $s$ could then be deduced from the actual magnet positions and their field lengths. The nominal value of $Z_{\text {bend }}$ was defined as that value of $Z_{\text {bend }}$ which yielded the most reconstructable events while minimizing the average $\chi^{2}$ of the track fit for the reconstructed events. This method determined $Z_{\text {bend }}$ to be $189.07 \mathrm{~cm}$ downstream of the upstream face of the first magnet or $3333.00 \mathrm{~cm}$ in the experiment coordinate system where $\mathrm{z}=0$ is the downstream face of magnet M1. Using this value of $Z_{\text {bend }}$ in Equation 6.3 defined $\lambda$ as 0.578 . These are the values shown in Table 6.1 and the ones used in the reconstruction program. The $\mathrm{P}_{\mathrm{T}}$ value shown in Table 6.1 was determined from $\mathrm{K}^{\circ} \rightarrow 2 \pi$ events. Requiring the $K^{\circ}$ rest mass to be $497.7 \mathrm{MeV} / \mathrm{c}^{2}$ defined $\mathrm{P}_{\mathrm{T}}$ as 1.554. This value of $\mathrm{P}_{\mathrm{T}}$ was used for 
all data tapes as no tape to tape variation was found in the $\mathrm{K}^{\circ} \rightarrow 2 \pi$ rest mass.

\begin{tabular}{|l|c|c|}
\hline Parameter & Units & Magnitude \\
\hline Magnet 1 Length ( $\ell$ ) & $\mathrm{cm}$ & 184.7 \\
Magnet 2 Length ( $\left.\ell^{\prime}\right)$ & $\mathrm{cm}$ & 188.8 \\
Gap (s) & $\mathrm{cm}$ & 44.5 \\
$\lambda=\mathrm{P}_{\mathrm{T}}(1) / \mathrm{P}_{\mathrm{T}}$ & & 0.578 \\
$\mathrm{Z}_{\text {bend }}$ & $\mathrm{cm}$ & 189.1 \\
$\mathrm{P}_{\mathrm{T}}$ & $\mathrm{GeV} / \mathrm{c}$ & 1.554 \\
\hline
\end{tabular}

Table 6.1 Magnet Parameters for E621

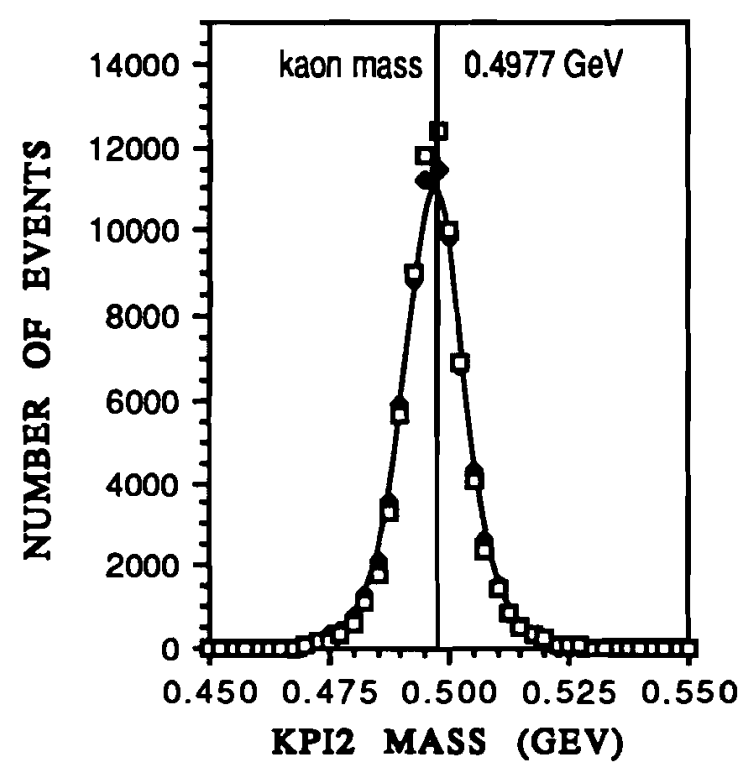

- data

- Monte Carlo

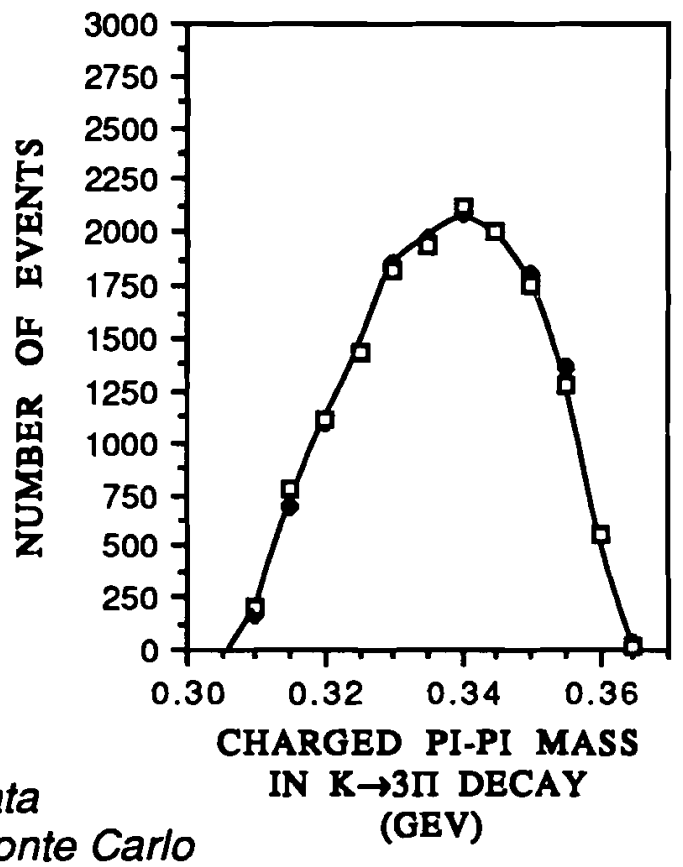

Figure $6.12 \pi+\pi-M a s s$

Figure $6.11 \quad K_{2} \pi$ Mass

First order $\theta$ and $\alpha$ corrections to $Z_{\text {bend }}$ as in equation 6.4 were made in the analysis program. These corrections were on the order of $0.05 \mathrm{~cm}$. The experiment 
following ours, E756, used the magnets M2 and M3 at the same current that E621 did and measured their magnetic fields. M2's maximum magnetic field in $y$ was $15.67 \mathrm{kG}$ and M3's was $11.39 \mathrm{kG}$. The effective field length's were measured as $189.7 \mathrm{~cm}$ and $191.6 \mathrm{~cm}$ for M2 and M3 respectively. These values of B and $\ell$ and $\mathrm{s}$ gave a value of 0.577 for $\lambda$, within $2 \%$ of the value determined from the E619 data. Figures 6.11 and 6.12 show the $K_{2 \pi}$ mass and the $\pi^{+} \pi^{-}$mass for Monte Carlo and data.

\subsubsection{Magnet Fringe Fields}

The C5y offset, which should show the greatest effect from fringe fieids showed no sign of them. Figure 6.13 shows MWPC C5y residual comparison plots on a logarithmic scale for data, Monte Carlo events with fringe fields as measured by E756 and Monte Carlo events without fringe fields for $\mathrm{K} \rightarrow 3 \pi$ decays.

I chose not to include fringe fields in my Monte Carlo since data and Monte Carlo comparisons showed no effect.

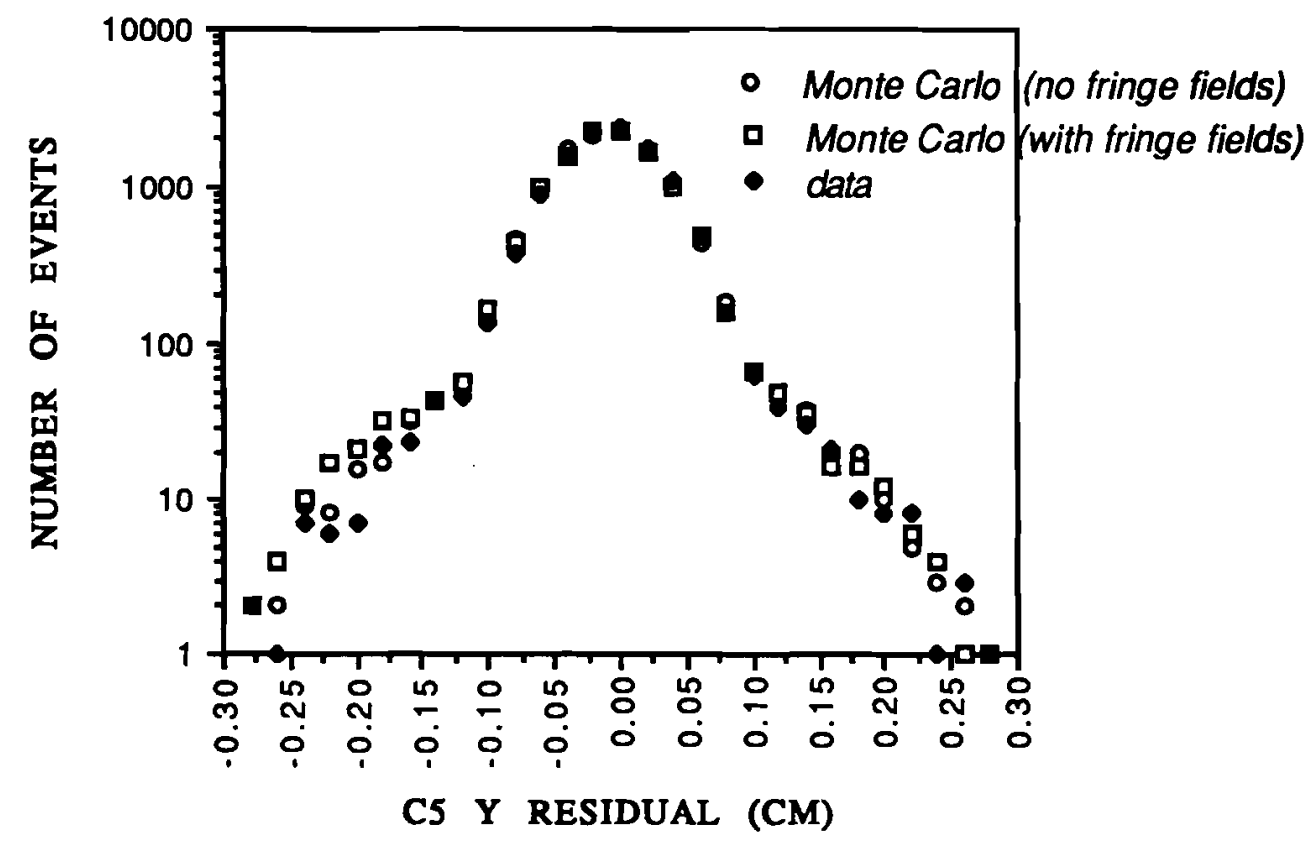

Figure 6.13 Fringe Fleld Comparlsons of C5 y Residual for $K \rightarrow 3 \pi$ Decays 


\subsection{Determining Lead Glass Calibration Constants}

The $K_{3 \pi}$ data were used to determine the lead glass block calibration constants on a tape by tape basis. Tape by tape calibration constants were needed to correct for the attenuation loss in the blocks due to the radiation damage.

The $\pi^{0}$ mass and the $\mathrm{K}^{0}$ mass were used as constraints in order to obtain the photon energies as in equations 6.5 and 6.6 .

$$
\begin{aligned}
& \mathbf{M}_{\pi^{\circ}}{ }^{2}=2 \omega_{1} \omega_{2}\left(1-\hat{k}_{1} \cdot \hat{k}_{2}\right) \\
& \mathbf{M}_{\mathrm{K}^{\mathrm{o}}}{ }^{2}=\overrightarrow{\mathrm{E}}_{\mathrm{K}^{\mathrm{o}}}{ }^{2} \cdot \overrightarrow{\mathrm{P}}_{\mathrm{K}^{\mathrm{o}}}{ }^{2} \\
& M_{K^{0}}{ }^{2}=\left(E_{v}+E_{\pi^{0}}\right)^{2}-\left(\vec{P}_{v}+\vec{P}_{\pi^{\circ}}\right)^{2} \\
& \mathrm{M}_{\mathrm{K}^{\mathrm{o}}}{ }^{2}=m_{\mathrm{v}}{ }^{2}+m_{\pi 0^{\circ}}{ }^{2}+2 \mathrm{E}_{\mathrm{v}}\left(\omega_{1}+\omega_{2}\right)-2 \overrightarrow{\mathrm{P}}_{\mathrm{v}} \cdot\left(\omega_{1} \hat{k}_{1}+\omega_{2} \hat{k}_{2}\right)
\end{aligned}
$$

$\omega_{1}, \omega_{2}$ are the photon energies, $\widehat{k}_{1}, \widehat{k}_{2}$ are the photon unit vectors from the decay vertex to the lead glass hit and $\overrightarrow{\mathrm{P}}_{\mathrm{v}}$ and $\mathrm{E}_{\mathrm{v}}$ are the four-vector components of the $\pi^{+} \pi^{-}$ combination with $m_{\mathrm{v}}$ and $m_{\pi^{\circ}}$ as the $\pi^{+} \pi^{-}$and $\pi^{\circ}$ mass. Equation 6.5 is a hyperbola of the form $\omega_{1} \omega_{2}=\mathrm{C}$ and Equation 6.6 is a line of the form $\omega_{2}=\mathrm{m} \omega_{1}+\mathrm{b}$. Thus there can be 0,1 or 2 solutions for $\omega_{1}$ and $\omega_{2}$. For the case where no solutions exist, a point half way between the two curves at their distance of closest approach was tried as a solution. In practice these events had poor $\pi^{\circ}$ masses. Typically there were two solutions to choose from. For the first iteration of the calibration constants the solution which gave the smallest kaon $\mathrm{P}_{\mathrm{T}}{ }^{2}$ (transverse momentum squared) at the target with respect to the neutral beam axis was chosen. Later iterations chose the solution with the smallest change in $\omega_{1}$ and $\omega_{2}$ from the previous measured photon energies.

For each event one determines the energy deposited in the lead glass array and then sums over events and blocks to determine a set of calibration constants for that tape. The energy in the $j^{\text {th }}$ block for the $i^{\text {th }}$ event, $\omega_{j i}$, is given by:

$$
\omega_{j i}=\mathrm{C}_{j} \mathrm{~A}_{i j}
$$

where $A_{i j}$ is the $A D C$ counts for that block and $C_{j}$ its calibration constant and $\omega_{j i}$ is 
determined by solving equations 6.5 and 6.6. Then the total energy $\mathrm{E}_{i}$ deposited in the array for a given event $i$ is:

$$
\mathrm{E}_{i}=\sum_{j} \omega_{j i}=\sum_{j} \mathrm{C}_{j} \mathrm{~A}_{j i}
$$

The calibration constants $C_{j}$ for each block were then determined by minimizing equation 6.9 where one sums over all events $i$ on a tape.

$$
\mathrm{F}=\sum_{i}\left(\mathrm{E}_{i}-\sum_{j} \mathrm{C}_{j} \mathrm{~A}_{j i}\right)^{2}
$$

The data sample needed to be $\pi^{\circ}$ decays. All the selection criteria discussed in Chapter 8 were used on this data sample to ensure that the decays were $\mathrm{K}^{\circ} \rightarrow \pi^{+} \pi^{*} \pi^{\circ}$. Several additional criteria were used to remove photon showers with poor energy or postion resolution. These are listed in Table 6.2. These three requirements removed $\sim 30 \%$ of the data.

\begin{tabular}{|l|c|}
\hline Extra Lead Glass Critera Used on Calibration Data & $\begin{array}{c}\text { Data } \\
\text { Lost }\end{array}$ \\
\hline (1) both photons greater than $5.58 \mathrm{~cm}$ from the hole & $10 \%$ \\
(2) no single block hit next to the hole & $23 \%$ \\
(3) no more than two neutral clusters in the glass & $15 \%$ \\
\hline
\end{tabular}

Table 6.2 Additional Requirements of the Callbration Constant Data Sample

For the six blocks around the hole in the center of the array two sets of calibration constants were used: one for events where the photon was found to be within $5.58 \mathrm{~cm}$ of the hole (from here on referred to as hole clusters) and one for events further from the hole but with energy in at least one of these six blocks. At first I only considered non-hole clusters and found calibration constants for all 86 
lead glass blocks. Then I did another iteration fixing these 86 constants and determining six new constants for the six blocks next to the hole for hole cluster events. Figure 6.14 displays typical glass block calibration constants tape by tape for the data used in this analysis. The top curve corresponds to a block next to the hole where radiation damage was high.

Some blocks near the edge of the array had few showers in them per data tape. Since these blocks were seldom hit they received little radiation damage and thus I took a weighted average of their calibration constants over the entire data set. These blocks were numbers $1-14,24,25,26,37,38,49,50,61,62,63$, and $73-86$ where the numbers correspond to those in Figure 3.6.

Using these calibration constants the average $\pi^{\circ}$ mass was $133 \mathrm{MeV}$ with a width of $17 \mathrm{Mev}$ as shown in Figure 6.15. The Monte Carlo simulation program results are also shown.

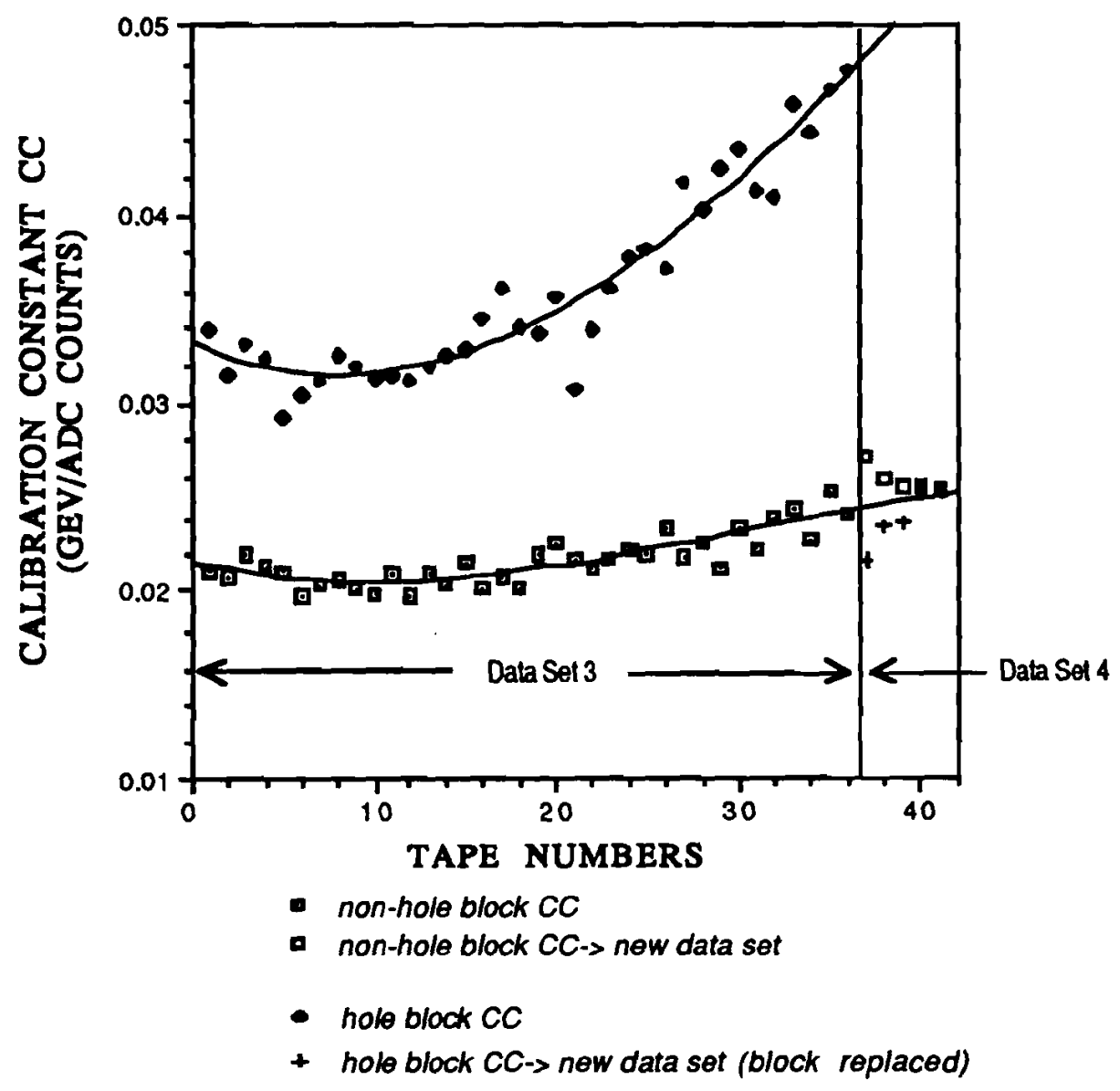

Figure 6.14 Varlations In Lead Glass Callbration Constants versus Time 


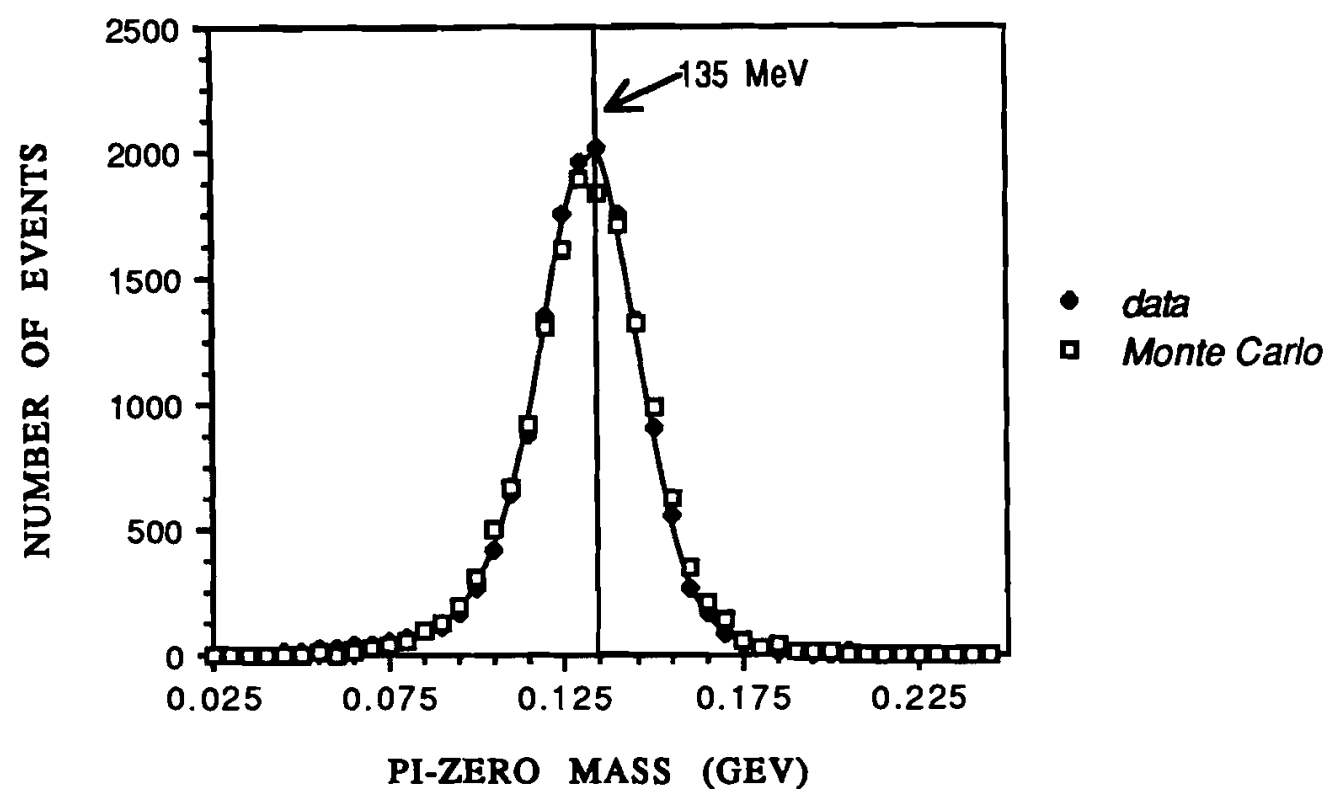

Flgure 6.15 PI zero Mass

\subsection{The Reconstruction Program}

\subsubsection{Introduction}

The reconstruction program seperated the data into three categories: $K^{0} \rightarrow 3 \pi$ candidates, $\mathrm{K}^{0} \rightarrow 2 \pi$ candidates and all reconstructable events. It identified an event as a $\mathrm{K}^{0} \rightarrow 2 \pi, \Lambda^{0} \rightarrow \mathrm{p} \pi$ or $\mathrm{K}^{0} \rightarrow 3 \pi$ candidate. $\mathrm{K}^{0} \rightarrow 3 \pi$ candidates were sent on to the lead glass reconstruction program which searched for two neutral showers with a $\pi^{\circ}$ mass in the lead glass array.

I will divide the discussion of the reconstruction program into two parts; one concerning the reconstruction of the charged tracks and the other the reconstruction of the two photons which shower in the lead glass array.

\subsubsection{Charged Track Reconstruction and Data Selection}

The decay topologies for $\mathrm{K}^{0} \rightarrow \pi^{+} \pi^{-} \pi^{0}$ and $\mathrm{K}^{0} \rightarrow \pi^{+} \pi^{-}$both require two charged tracks which diverge from a common decay point and are then bent through opposite angles in the analysis magnets. A common decay point was found in both the $x$ and $y$ views. The reconstruction program used the least squares method to fit the MWPC wire hits to straight lines. In the $y$-view the charged particles were not bent and 
thus all $y$-plane chamber hits were tried in order to determine the best straight lines. If only two tracks were found in the $y$-view, then the program tried to fit the $x$ wire hits upstream of the analysis magnets, using the uv chamber $C 2$, which was tilted at $45^{\circ}$, to determine which $x$ track corresponded to which $y$ track. Downstream of the analysis magnets the $x$ tracks were required to intersect the upstream $x$ tracks at the magnet bend plane. The determination of this bend plane was discussed in Section 6.3.

The decay vertex was constrained to have the same $z$ in both the $x$ and $y$ views. A geometrical $\chi^{2}$ was calculated for each event's track fit. Those events with $\chi^{2} / \mathrm{DOF}$ $<3.0$ were kept, where DOF is the number of degrees of freedom in the fit. This process defined upstream and downstream $\mathrm{x}$ view slopes with respect to the analysis magnets, one set of $y$ slopes, a decay vertex point and a $z$ bend plane point for the charged tracks. The momenta of the charged tracks were then determined by the difference between the $\mathrm{x}$ view slopes upstream and downsteam of the analysis magnets. From these momenta and slopes one then could determine a neutral vee mass for the two charged tracks in order to determine the parent neutral particle.

Two scenarios were tried to determine the identity of the parent particle. The two charged tracks were reconstructed as a proton (p) and a pion ( $\pi$ ) and as two pions as shown in Equation 6.10.

$$
\mathrm{M}^{2}=m_{+}^{2}+m_{-}^{2}+2 \mathrm{E}_{+} \mathrm{E}_{-}-2\left(\overrightarrow{\mathrm{p}}_{+} \cdot \overrightarrow{\mathrm{p}}_{-}\right)
$$

The masses $\left(m_{+}, m_{-}\right)=\left(m_{\pi^{+}}, m_{\overline{\mathrm{p}}}\right),\left(m_{\mathrm{p}}, m_{\pi^{-}}\right)$or $\left(m_{\pi^{+}}, m_{\pi^{-}}\right)$.

The two charged particles from a $\mathrm{K}^{0} \rightarrow 3 \pi$ decay do not reconstruct to a unique mass due to the missing $\pi^{\circ}$ mass, yet they must reconstruct to a mass of at least two times the pion mass and not more than the kaon mass minus the $\pi^{0}$ mass. This restricts the $\mathrm{K}^{0} \rightarrow 3 \pi \pi^{+} \pi^{-}$mass to between $0.280 \mathrm{GeV} / \mathrm{c}^{2}$ and $0.3675 \mathrm{GeV} / \mathrm{c}^{2}$. Thus a two pion mass between $0.280 \mathrm{GeV} / \mathrm{c}^{2}$ and $0.3675 \mathrm{GeV} / \mathrm{c}^{2}$ was identified as a $\mathrm{K}_{3 \pi}$ candidate and a two pion mass greater than $0.425 \mathrm{GeV} / \mathrm{c}^{2}$ was identified as a $\mathrm{K}_{2 \pi}$ candidate. A proton-pion mass of $1.1156 \pm 0.010 \mathrm{GeV} / \mathrm{c}^{2}$ was identified as a lambda candidate. Some charged particles had ambiguous charged masses in that they satisfied both the $\mathrm{K}_{2 \pi}$ and lambda candidate requirements. These events were not kept in the $\mathrm{K}_{\mathbf{2} \pi}$ data sample. 


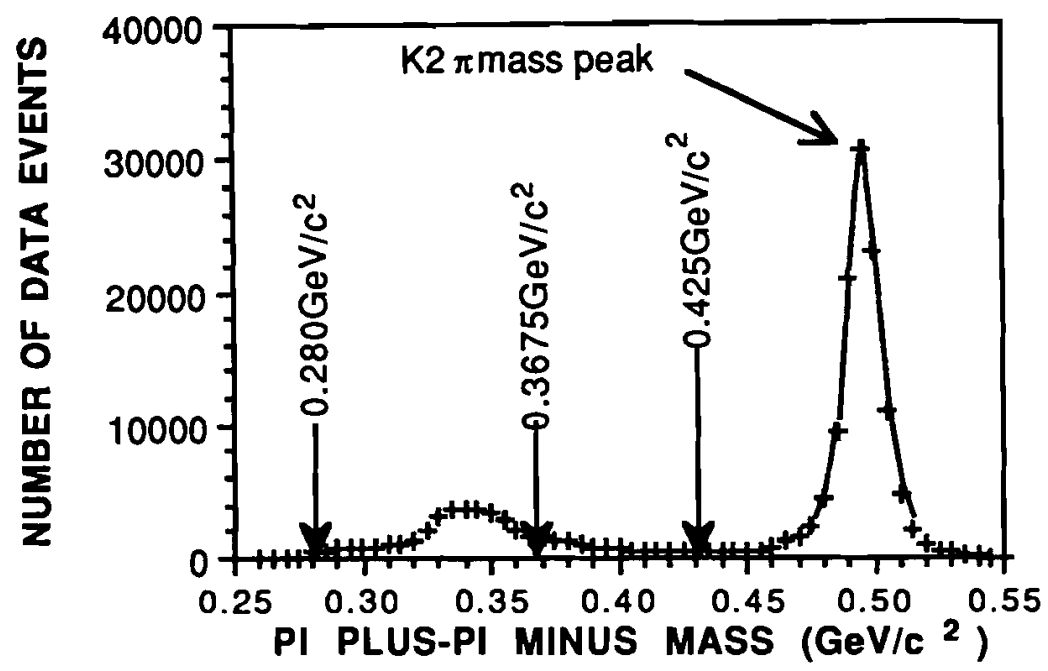

Figure $6.16 \pi \pi$ Invarlant Mass

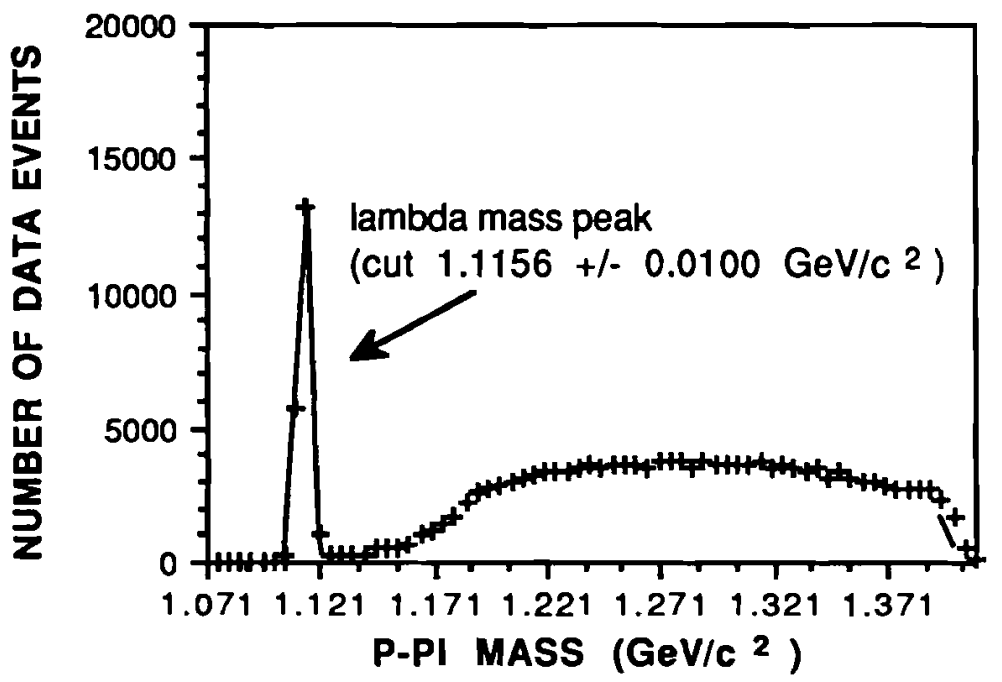

Figure $6.17 \pi p$ Invariant Mass

Figure 6.16 shows the $\pi^{+} \pi^{-}$invariant mass and Figure 6.17 the $\pi p$ invariant mass from $K_{3 \pi}$ triggers. The $\Lambda^{0}$ and $K^{0} \rightarrow 2 \pi$ mass peaks are clearly visible and the mass selection criteria are indicated on the graphs. The $K^{0} \rightarrow 3 \pi$ candidates $\pi^{+} \pi^{-}$ invariant mass is also visible and its mass cuts are shown. Table 6.2 shows what 
fraction of the $\mathrm{K}_{3 \pi}$ and $\mathrm{K}_{2 \pi}$ trigger events reconstructed to a vertex. Included in Table 6.2 is the fraction of the events that reconstructed to a vertex whose charged mass corresponded to a $\Lambda^{0}, K^{0} \rightarrow 3 \pi$ or $\mathrm{K}^{0} \rightarrow 2 \pi$ candidate. Less than $0.05 \%$ of the reconstructable vees from $K_{2 \pi}$ trigger events passed all the $K_{3 \pi}$ final selection criteria versus $2.2 \%$ of the $\mathrm{K}_{3 \pi}$.trigger events.

\begin{tabular}{|l|c|c|c|}
\hline Category & $\begin{array}{c}\mathrm{K} \rightarrow 2 \pi \text { Triggers } \\
\text { (data) }\end{array}$ & $\begin{array}{c}\mathrm{K} \rightarrow 3 \pi \text { Triggers } \\
\text { (data) }\end{array}$ & $\begin{array}{c}\mathrm{K} \rightarrow 3 \pi \text { Triggers } \\
\text { (Monte Carlo) }\end{array}$ \\
\hline Reconstruct to a "vee" & $69 \%$ & $59 \%$ & $88 \%$ \\
$\mathrm{~K} \rightarrow 2 \pi$ candidate & $66 \%$ & $71 \%$ & $0.4 \%$ \\
$\Lambda$ candidate & $22 \%$ & $11 \%$ & $0.4 \%$ \\
$\mathrm{~K} \rightarrow 3 \pi$ candidate & $11 \%$ & $16 \%$ & $99.2 \%$ \\
Ambiguous $\mathrm{K} \rightarrow 2 \pi$ or $\Lambda$ & $1 \%$ & $2 \%$ & $0 \%$ \\
\hline
\end{tabular}

Table 6.3 First Order Reconstruction of $K_{3 \pi}$ and $K_{2 \pi}$ Triggers

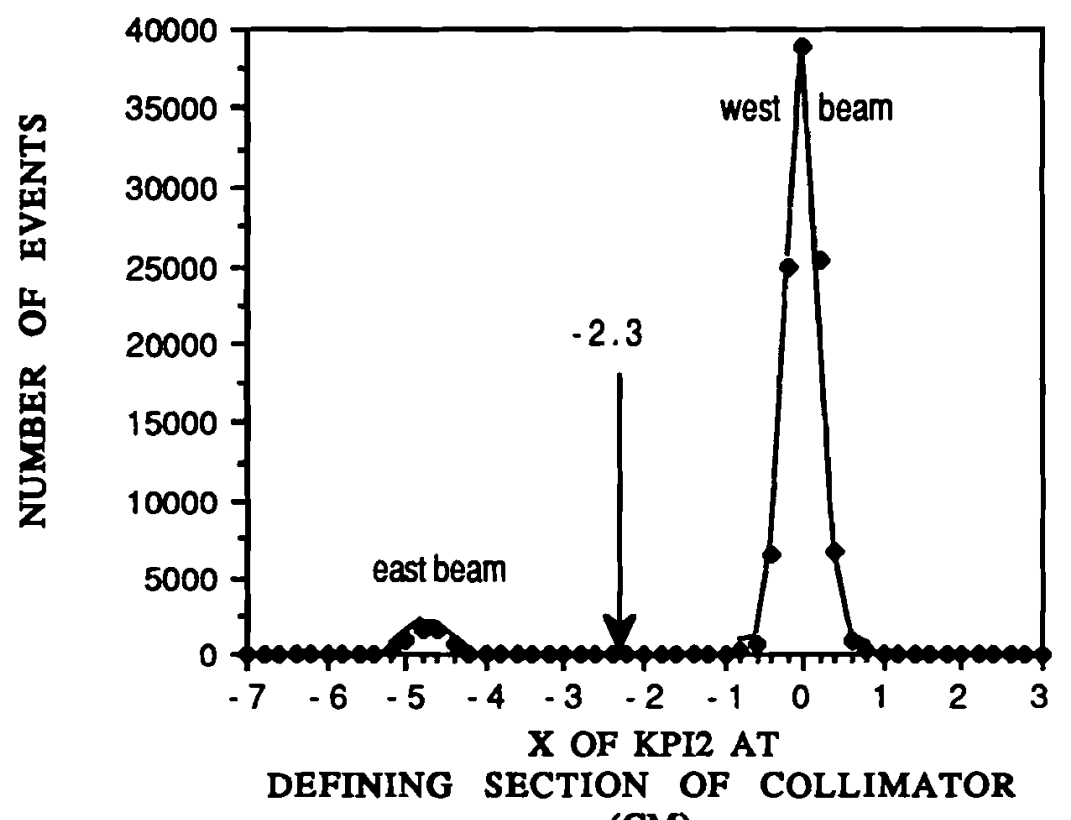

(CM)

Figure 6.18 Projection to Collimator Defining East and West: $K \rightarrow 2 \pi$ Data 
The $\mathrm{K}_{2 \pi}$ momentum vector was projected back to the defining section of the collimator in order to determine which beam the kaon came from. This plot and the data selection defining east and west is shown in Figure 6.18.

\subsubsection{Photon Shower Reconstruction}

The photon reconstruction program searched for the largest signal in the lead glass array and called it the central block of the first cluster. It then checked to see how many of the block's nearest neighbors also had signals. The sum of the calibrated signals from these hit blocks was taken to be the energy of the shower. Once all the blocks in the shower were determined they were removed from the data array and the next largest signal in the array was found. This process continued until all hit blocks were accounted for. The characteristics of the photon showers for $\mathrm{K}_{3 \pi}$ events are given in Table 6.4 and in Figures 6.19 through 6.21.

Photon 1:

(higher energy gamma)

(1) average radius 1.0 block (one block is $10.16 \mathrm{~cm}$ )

(2) average energy $28 \mathrm{GeV}$, sigma of $15 \mathrm{GeV}$

(3) peak energy

$21 \mathrm{GeV}$
Photon 2:

(lower energy gamma)

1.0 block

$14 \mathrm{GeV}$, sigma of $5 \mathrm{GeV}$

$9 \mathrm{GeV}$

$41 \%$ both photons away from the hole (more than $5 \mathrm{~cm}$ away

$47 \%$ at least one photon near the hole

$11 \%$ both photons near the hole

$5.4 \%$ less blocks in a shower at end of a data set due to radiation damage

Table 6.4 General Photon Stat/stlcs 


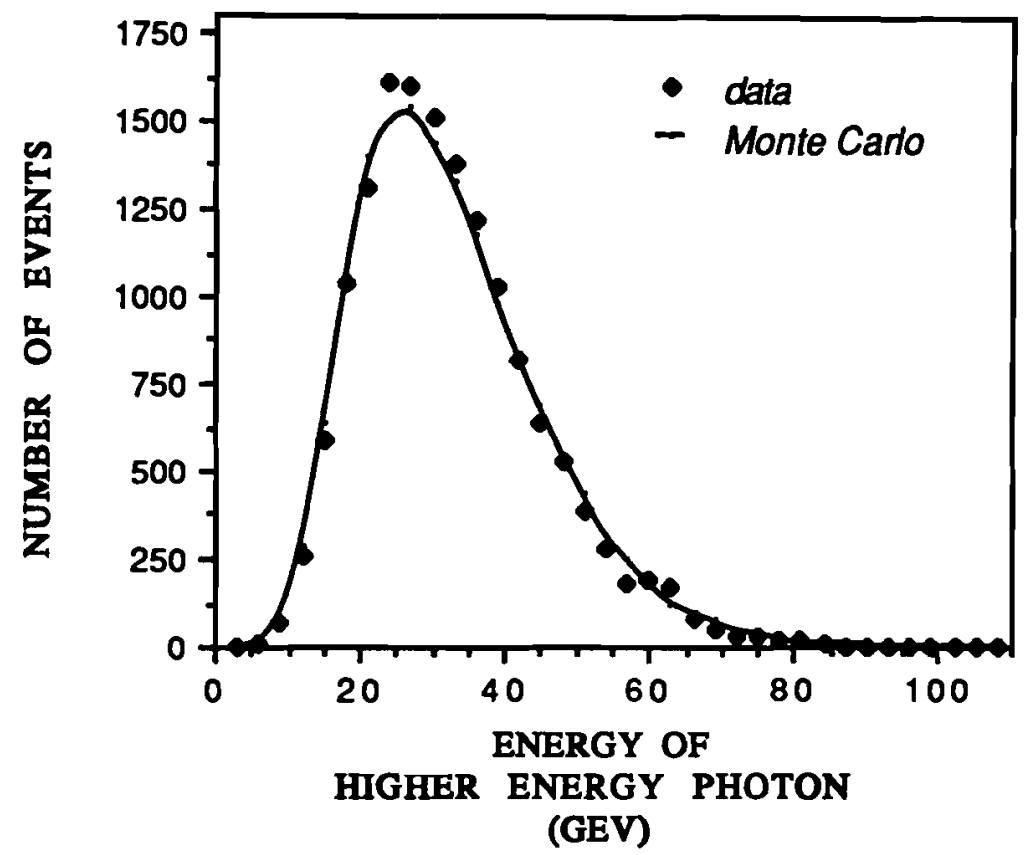

Flgure 6.19 Higher Energy Photon Energy

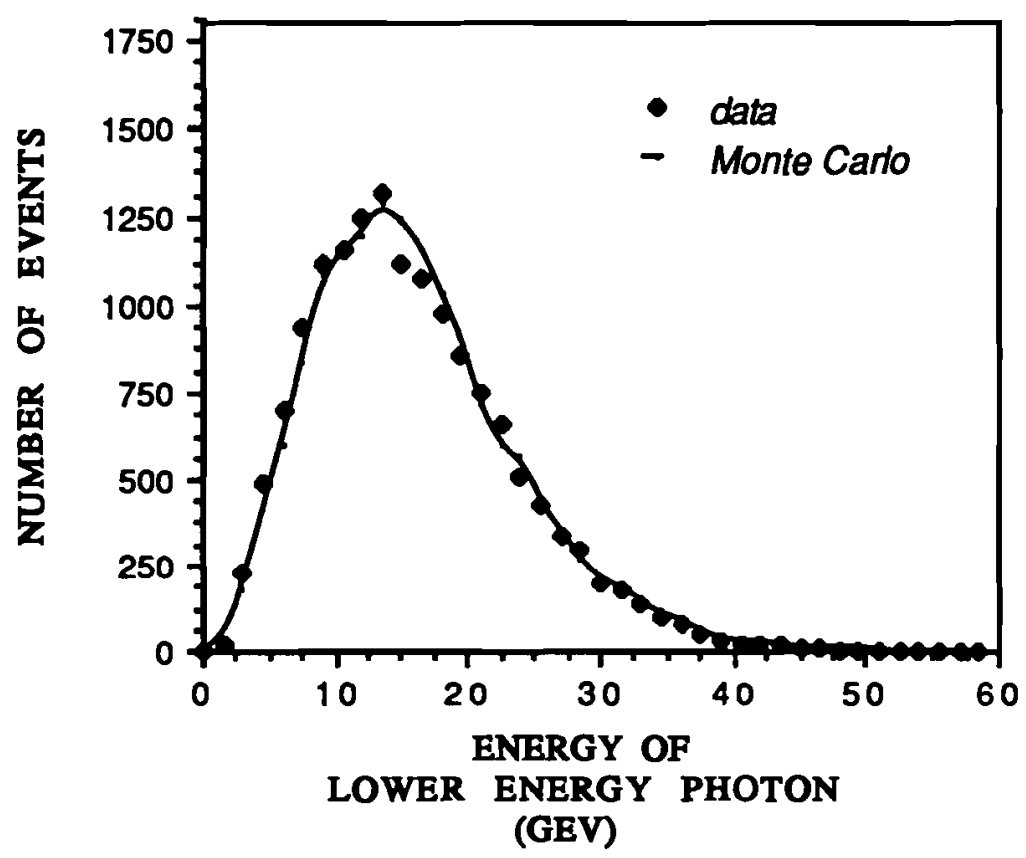

Flgure 6.20 Lower Energy Photon Energy 


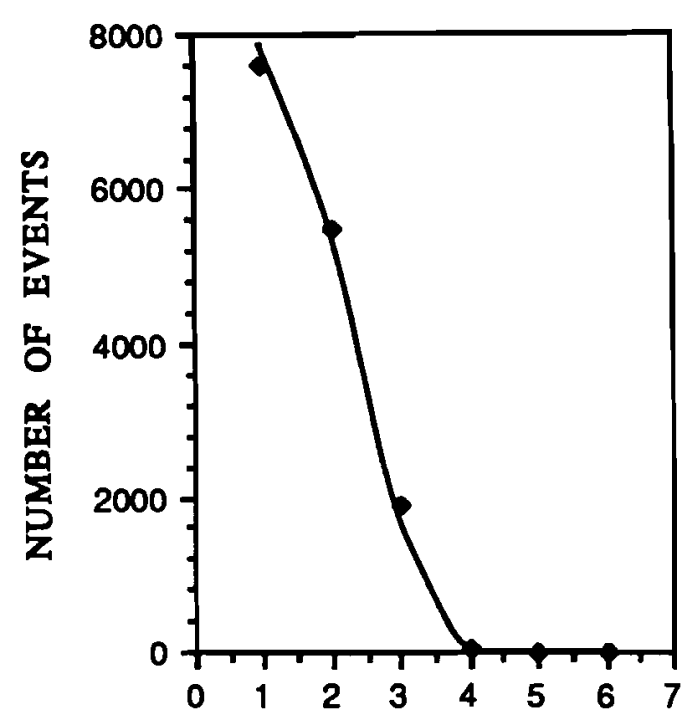

NUMBER OF BLOCKS:

HIGHER BNERGY SHOWER (one block $\sim 10 \mathrm{~cm}$ )

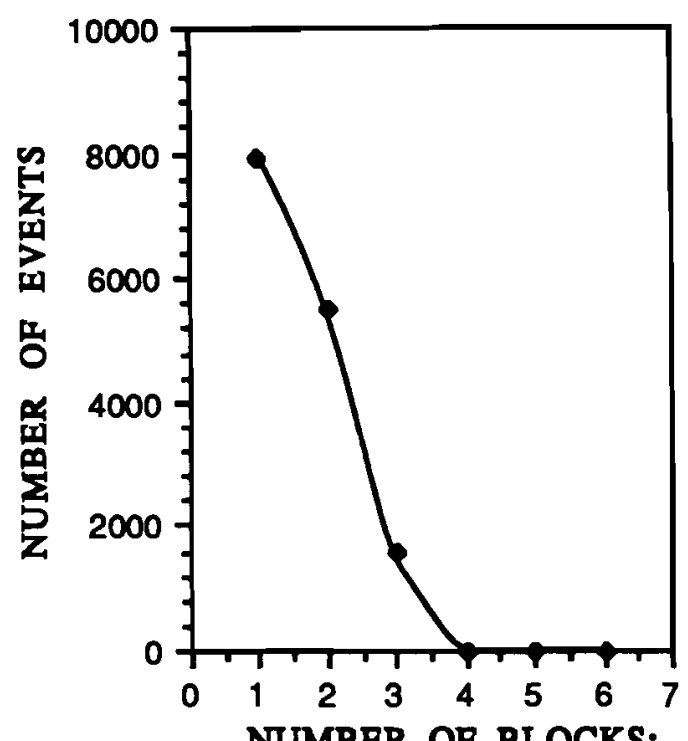

NUMBER OF BLOCKS: HIGHER ENERGY SHOWER (one block $\sim 10 \mathrm{~cm}$ )

- Monte Cano

Flgure 6.21 Photon RadII

Any blocks with out of time signals as determined by the TDC's were ignored, and an energy threshold of $>1.0 \mathrm{GeV}$ for a block was required. Plots of TDC counts for each block were used to determine average TDC times for each block and their variations from the average times. I made a file of GCF trigger processor TDC and regular TDC average times for all blocks tape by tape. The GCF trigger processor TDC's were all usually within 12 nsec of their central value. Regular TDC average times varied more widely but were usually within $15 \mathrm{nsec}$ of their average time. These TDC times and ranges were used to remove out of time events.

A 'good' lead glass block signal was defined as:

(1) $>1.0 \mathrm{GeV}$ (approximately 35 ADC counts)

(2) GCF trigger processor TDC within 12 nsec of the in time average for that block

(3) Regular TDC within 15 nsec of the in time average for that block

Next the program determined which clusters were caused by charged particles hitting the lead glass array. At least one charged pion hit the lead glass array $50 \%$ of the time and both charged pions hit the lead glass array $16 \%$ of the time. When the 
charged pions did hit the array they were usually near the outside of the array whereas most of the photons from the neutral pions were near the center. Figure 6.22 is a plot of the distance from the charged track to the nearest glass cluster center for both Monte Carlo and data $K_{3 \pi}$ events (one block width $\sim 10 \mathrm{~cm}$ ). The Monte Carlo has no spike of events at zero since it does not simulate charged showers. There is also an excess of data events in the neutral shower peak to the near side of the charged pion hit. Charged pion showers can be quite large and extend beyond nearest neighbor blocks. Since the program only considers nearest neighbor blocks as a shower, the extra hit blocks in the charged pion shower are identified as another shower, usually a neutral one due to its distance from the center of the charged pion shower. To insure that these "extended" charged pion showers do not lose good pi-zero events due to their proximity to a "good" neutral shower, a plot of the smallest distance from a pion track to a chosen photon shower center (one which reconstructs to a good pi-zero mass) is shown in Figure 6.23 for Monte Carlo and data. The Monte Carlo and data graphs match well showing few events are lost and thus the lack of charged showers in the Monte Carlo is not a problem.

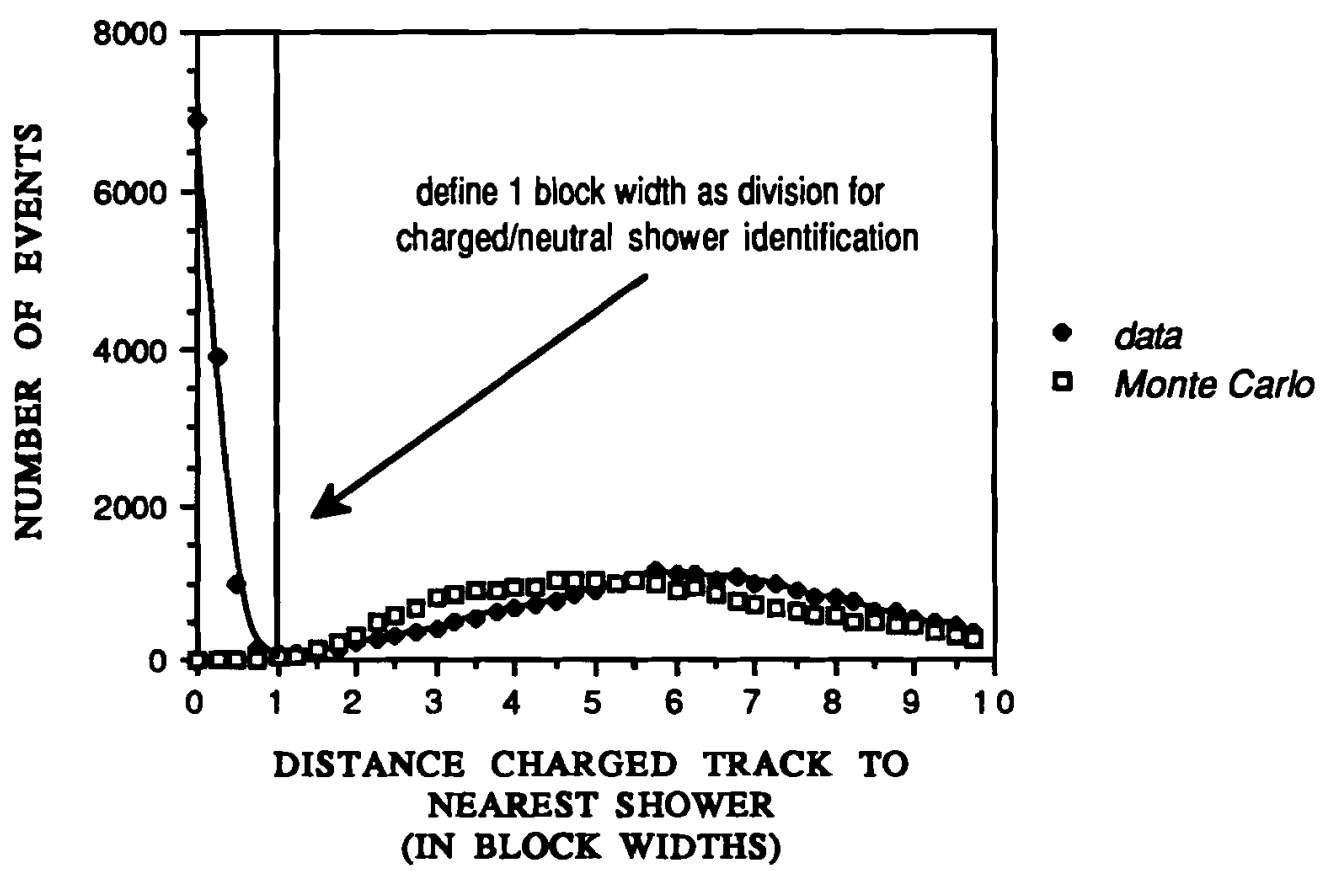

Flgure 6.22 Distance From the Plon track to the Nearest Shower Center 


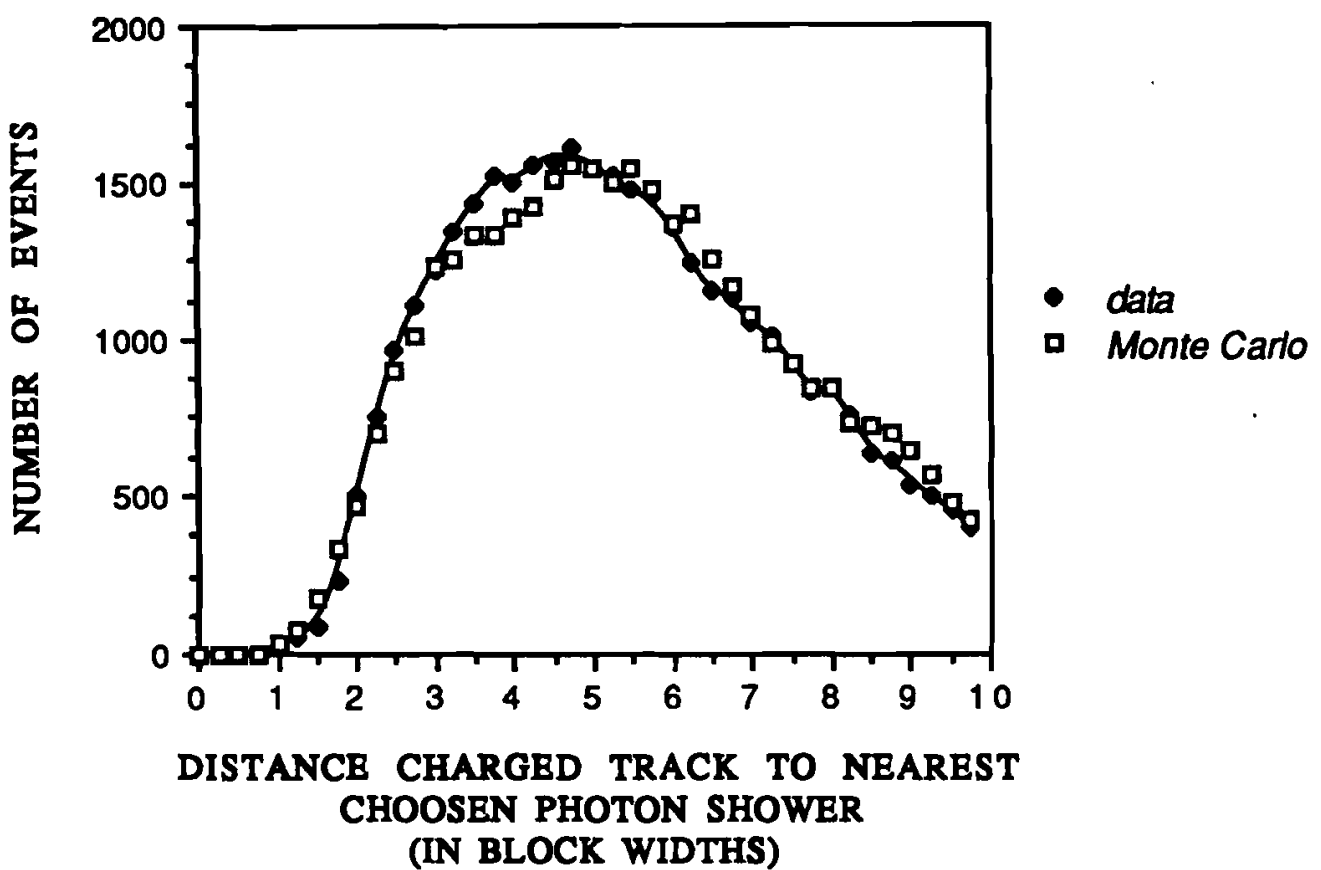

Flgure 6.23 Smallest Dlstance From a Plon track to a Chosen Photon Shower center

Any charged track within $10 \mathrm{~cm}$ or 1 block width of a cluster center caused that cluster to be removed as a photon candidate in the analysis program. Also, if the $\mathrm{Pi}$ hodoscopes had a hit in a overlap region near the cluster such that the central block of the cluster was vetoed as in Figure 4.13, the cluster was removed as a photon candidate.

A $\pi^{\circ}$ mass was determined for all combinations of neutral clusters by assuming that the two photons originated from the charged track vertex. The $\pi^{0}$ mass was found using equation 6.11, where $E_{\gamma_{1}}, E_{\gamma_{2}}$ are the energies of the two photon showers and $\theta$ the angle between their vectors determined by projecting from their glass position to the charged particle decay vertex.

$$
M_{\pi^{o}}=2 E_{\gamma_{1}} E_{\gamma_{2}}(1-\cos \theta)
$$


The expression for $\cos \theta$ is given in equation 6.12 where $\left(x_{\gamma_{1}}, y_{\gamma_{1}}, z_{\gamma_{1}}\right)$ and $\left(x_{\gamma_{2}}, y_{\gamma_{2}}, z_{\gamma_{2}}\right)$ are the coordinates of the two photon showers in the lead glass array relative to the vertex of the decay i.e . $x_{\gamma_{1}}=x_{\gamma_{1}}^{\prime}-x_{\text {vertex }}$ where $x_{\gamma_{1}}^{\prime}$ is the calculated $x$ position of the shower.

$$
\cos \theta=\frac{x_{\gamma_{1}} \cdot x_{\gamma_{2}}+y_{\gamma_{1}} \cdot y_{\gamma_{2}}+z_{\gamma_{1}} \cdot z_{\gamma_{2}}}{\sqrt{\left(x_{\gamma_{1}}^{2}+y_{\gamma_{1}}^{2}+z_{\gamma_{1}}^{2}\right) \cdot\left(x_{\gamma_{2}}^{2}+y_{\gamma_{2}}^{2}+z_{\gamma_{2}}^{2}\right)}}
$$

The photon shower positions were determined from the first moments of the block energies and corrected with electron-positron position functions as discussed in 6.2.6. The two photon showers with the best $\pi^{0}$ mass were then chosen for reconstructing the full $\mathrm{K}_{3 \pi}$ event. The extra neutral clusters usually were very low in energy and single block showers. Looking at these events showed that they were pieces of a larger shower that extended beyond nearest neighbor blocks. As mentioned previously, these large extended showers were often caused by the charged

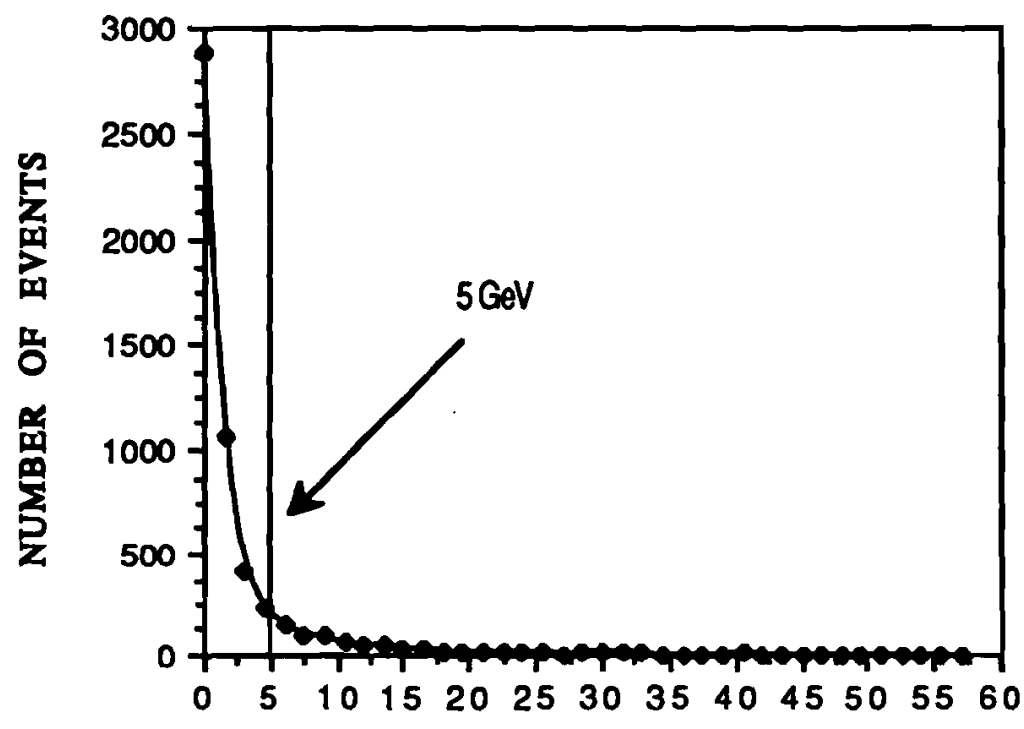

ENERGY OF EXTRA NEUTRAL SHOWER (GEV)

Flgure 6.24 Energy of the Highest Energy Extra Neutral Shower 
pions hitting the lead glass array. I chose to remove events in which there was an extra neutral shower with energy greater than $5 \mathrm{GeV}$. $16 \%$ of the data had more than two neutral showers and only $18 \%$ of those showers had energies greater than $5 \mathrm{GeV}$. Figure 6.24 shows the energy distribution of these extra neutral showers. The Monte Carlo has no events in this classification.

Using all the charged pion vectors and the two gamma vectors, a $K_{3 \pi}$ mass and momentum vector were determined.

and

$$
\mathrm{M}_{\mathrm{K} 3}=\sqrt{\mathrm{M}_{\pi \pi}^{2}+\mathrm{M}_{\pi^{\mathrm{o}}}^{2}+2\left[\mathrm{E}_{\pi \pi}\left(\mathrm{E}_{\gamma 1}+\mathrm{E}_{\gamma 2}\right)-\left(\overrightarrow{\mathrm{P}}_{\pi \pi} \cdot \overrightarrow{\mathrm{P}}_{\pi^{\circ}}\right)\right]}
$$

where

$$
\mathrm{P}_{\mathrm{K} 3}=\sqrt{\mathrm{P}_{x}^{2}+\mathrm{P}_{y}^{2}+\mathrm{P}_{z}^{2}}
$$

$E_{\pi \pi}$ and $M_{\pi \pi}$ are the energy and mass of the charged pion system, $E_{\gamma_{1}}$ and $E_{\gamma_{2}}$ are the two photon shower energies, $M_{\pi^{\circ}}$ is the $\pi^{0}$ mass, $\vec{P}_{\pi \pi}$ and $\vec{P}_{\pi^{0}}$ are the charged pion system and neutral pion momentum vectors, and $\mathrm{u}_{\gamma 1 i}$ and $\mathrm{u}_{\gamma_{i} i}$ are the photon unit vectors projected on the axis $i$. The $\mathrm{K}_{3 \pi}$ momentum vector from equation 6.14 projected back to the defining section of the collimator determined which target the

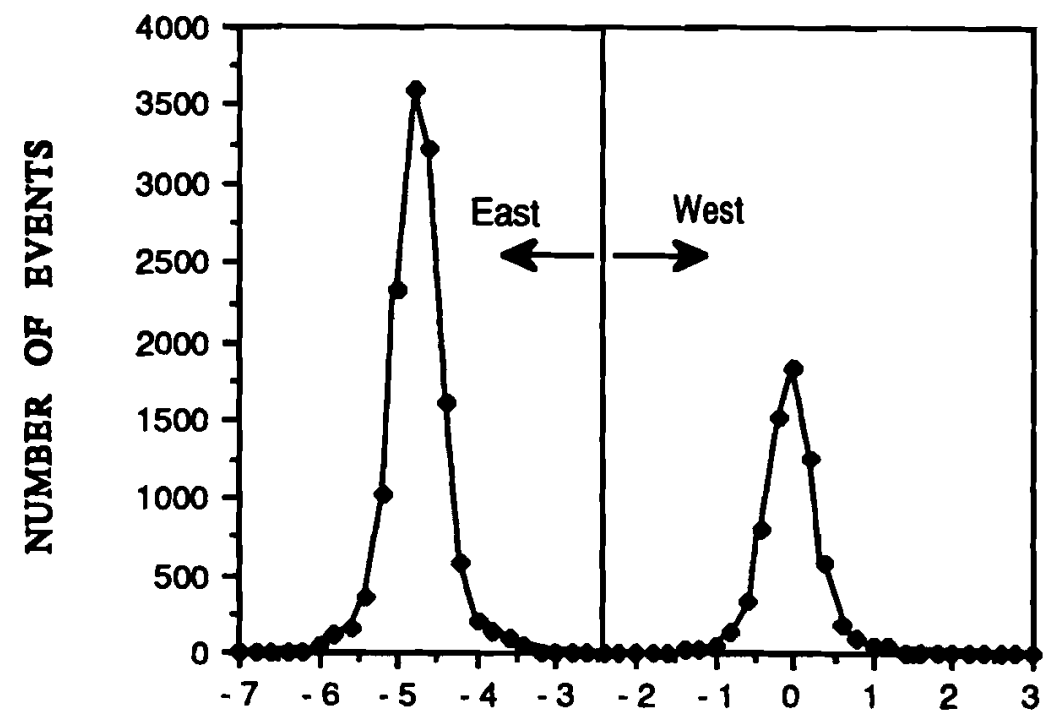

$X$ POSITION AT DEFINING SECTION OF COLLIMATOR (CM)

Figure 6.25 Collimator Cut Defining East and West for $K_{3 \pi}$ Data 
kaon came from. The line defining east and west is shown in Figure 6.25, where the $x$ projection of the kaon at the defining section of the collimator is shown for $K_{3 \pi}$ data.

\subsubsection{X,Y Position of Photon Showers}

The position of a photon shower was determined from the first moments of the cluster using the energy in a block and its center position as in equation 6.15 where $\mathbf{x}_{i}$ is the center position of block $i, \mathrm{E}_{i}$ is the energy in block $i$ and $\mathrm{E}_{\text {total }}$ is the total energy in the shower.

$$
x=\sum_{i} \frac{\mathrm{x}_{i} \mathrm{E}_{i}}{\mathrm{E}_{\text {total }}}
$$

Since actual shower positions could be determined from the $\mathrm{e}^{+} \mathrm{e}^{-}$tracks through the chambers, correction factors for the positions calculated using equation 6.15 could be determined. These correction factors would be different for showers near the hole in the center of the array and showers more than 1/2 a block width away since energy lost in the hole in the array would effect the shower position as calculated with equation 6.15 .

All crude (i.e. uncorrected) and actual shower positions (as determined from tracks) were measured as an absolute distance from the center of the block with the largest signal. Figure 6.26 is a plot of the crude position as determined by equation 6.16 versus the actual track position at the lead glass array for $x$ and $y$. The corrected positions are shown also in the figures. The $45^{\circ}$ line drawn corresponds to the desired condition where the position determined from the glass energies is the same as that determined from the track. The units are half block widths such that 0 corresponds to a photon hit in the center of a block and 1 to a hit on the edge and .2 block widths is approximately $1 \mathrm{~cm}$. The data was divided into two categories, nonhole shower data and hole shower data. Non-hole shower data coresponded to events where both photon positions were calculated to be more than $5.08 \mathrm{~cm}$ from the hole in the center of the array. Hole data corresponded to both photon showers being within $5.08 \mathrm{~cm}$ of the hole. These two categories were chosen due to the energy and position resolution differences between them. Only non-single block hit events were used in the calculations. For both categories, curves like the one shown in Figure 6.26 determined correction factors for the crude positions. The same correction factors were used for the entire data sample. 


\subsubsection{Non-Hole Photon Showers}

Figure 6.26 shows the crude and corrected shower positions for non-hole events. The correction factors calculated from the curve improved the position resolution to \pm 0.02 block widths or $0.1 \mathrm{~cm}$. The same correction factors were used for all showers whose crude positions categorized them as non-hole showers.

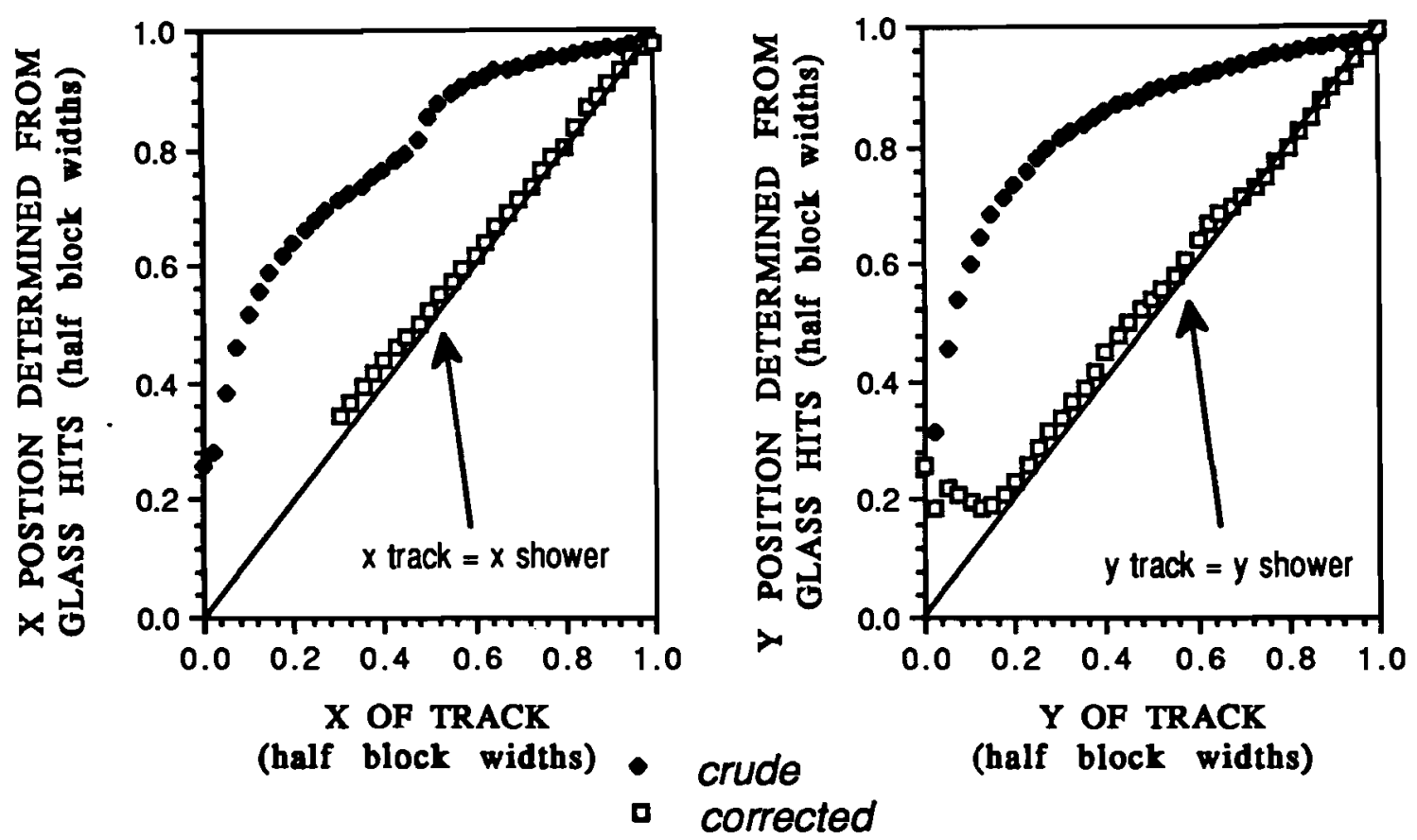

Figure 6.26 Uncorrected \& Corrected Non-hole Positions vs Track Positions

\subsubsection{Hole Photon Showers}

Since $60 \%$ of the $\mathrm{K}_{3 \pi}$ data had a photon shower within $1 / 2$ a block of the hole, it was important to reconstruct these events accurately. Correction factors were found for the three regions around the hole in the center of the array shown in Figure 6.27. Showers in region 3 behaved as the non-hole showers of Figure 6.26 and thus the non-hole correction factors were used for these events with similar results.

In region 1 of Figure 6.27 , the $y$ correction factors were similar to the ones found for non-hole events. On the other hand the $x$ positions were poorly determined due to the hole and nearly impossible to correct with $\mathrm{e}^{+} \mathrm{e}^{-}$data. All bins in terms of $x$ track had $\mathrm{x}$ crude peaking at zero with a high tail. 


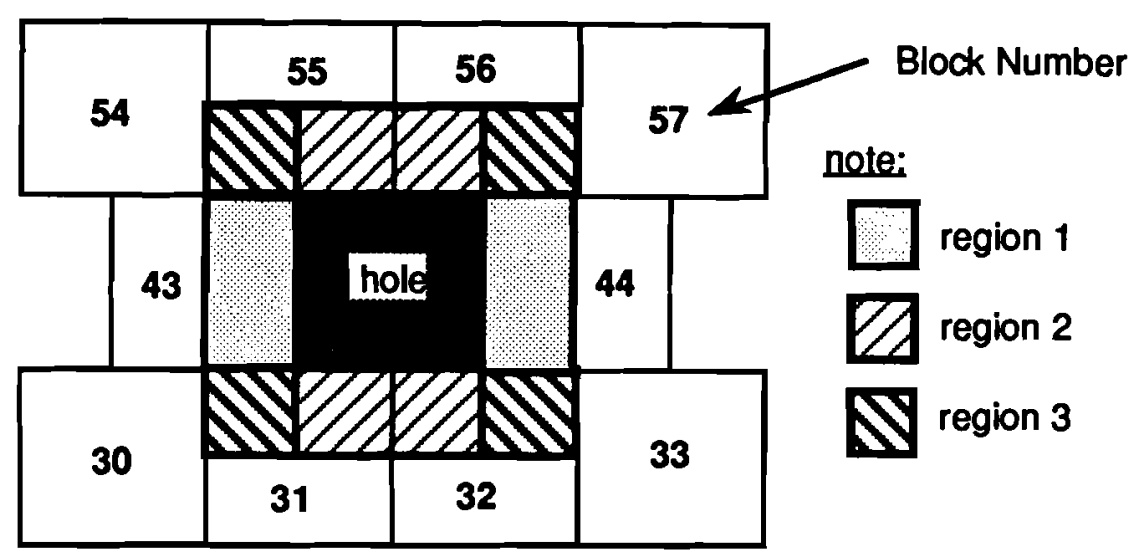

Figure 6.27 Hole Reglons

Little could be done to correct the $\mathrm{x}$ positions. The correction factors merely distributed the events more evenly across the block. The final $\mathbf{x}$ position determination was good to $\pm 2.5 \mathrm{~cm}$ as shown by the corrected track position versus actual track graph in Figure 6.28. Photons which showered only in the block next to the hole were given a crude position of 1.0 or the block center. In most cases where a photon showered in a block next to the hole, little energy spread to the neighboring blocks near the hole and thus the crude positions never got closer than 0.6 half block widths $(3 \mathrm{~cm})$ to the hole. Also, since any showers which spread to a neighborring block further from the hole would usually be reconstructed to a non-hole shower, few hole showers reconstructed to a position close to the center of the block (0 half block widths, $5 \mathrm{~cm}$ from the hole). The $y$ resolution, after using the same correction factors as those determined for non-hole events, was $\pm 0.3 \mathrm{~cm}$. Figure 6.28 also shows the corrected y glass positions.

For region 2 of Figure 6.27, the situation was similar to that of region 1 only with $\mathrm{x}$ being well determined and $\mathrm{y}$ not. The $\mathrm{x}$ non-hole correction factors worked fine for the $x$ position (see Figure 6.29), but the $y$ postion needed a different set of correction factors. The y crude versus y track position was scattered, but upon examining the individual bins of the distribution one saw a peak offset from the main spike at zero. This peak was correlated to the track position and thus the correction factors determined for region 2 y did give some improvement in the position resolution. The original and corrected $y$ plots and the corrected $x$ plots are in Figure 6.29 for region 2. 


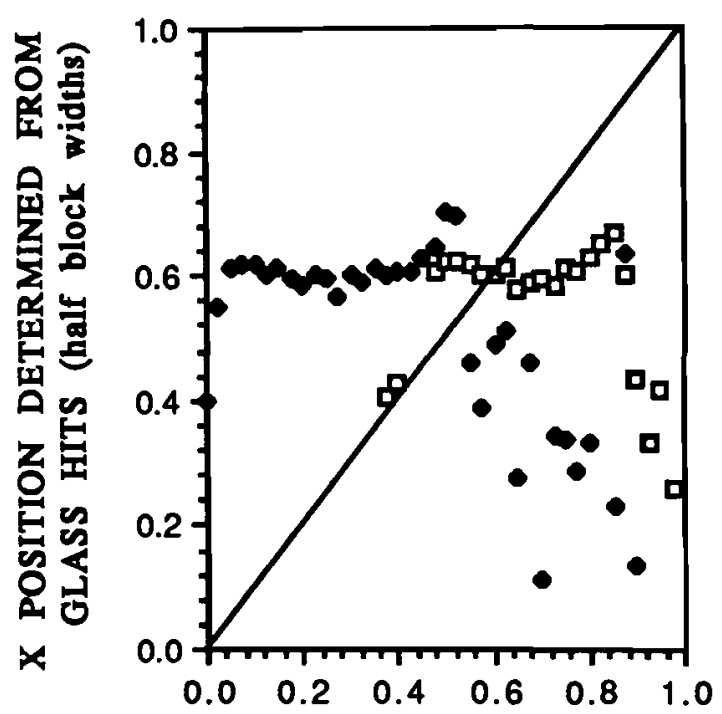

$X$ OF TRACK

(half block widths) - crude

- corrected

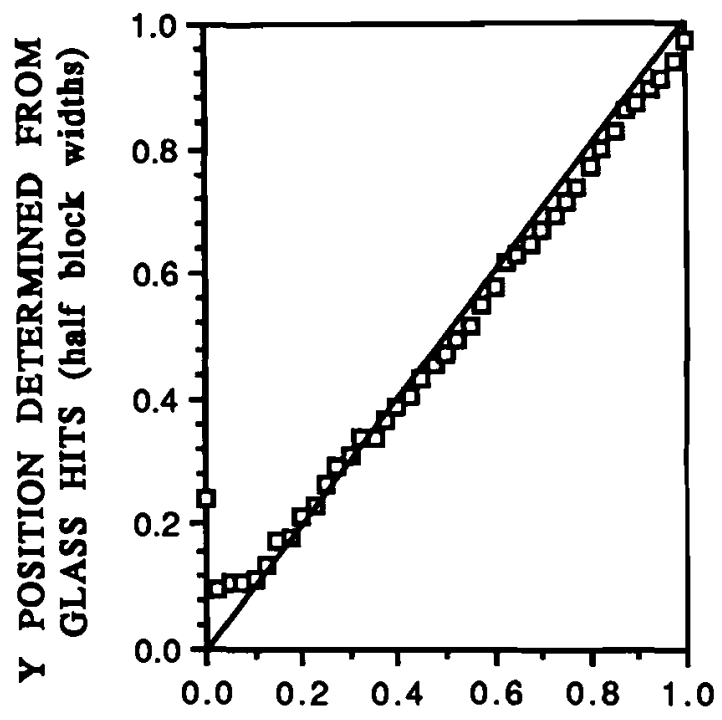

Y OF TRACK

(half block widths)

Figure 6.28 Reglon 1 Corrected Positlons vs. Track Positions

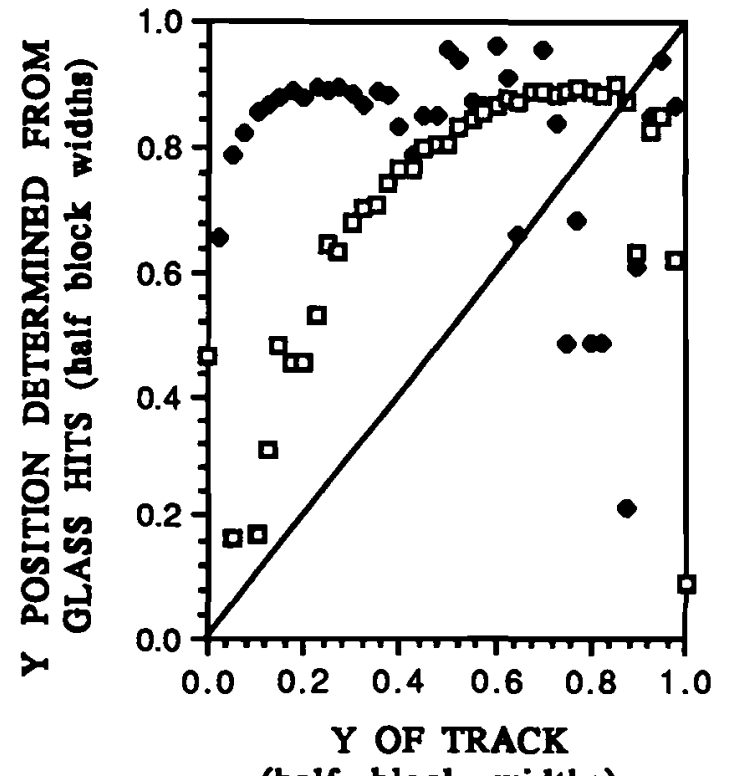

(half block widths)

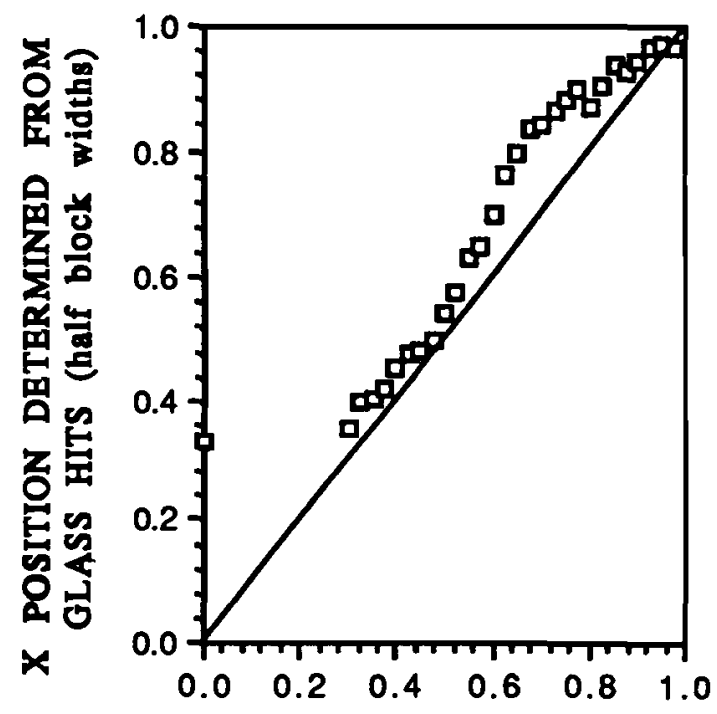

$X$ OF TRACK

(half block widths)

- corrected

Figure 6.29 Reglon 2 Corrected Positions vs. Track Positions 


\section{Chapter Seven \\ The Monte Carlo}

\subsection{Introduction}

In measuring $\eta_{+-0}$, the quantities needed are the number of kaons which decay in the decay volume as a function of proper time. Many kaons which decay in the decay region are never recorded on tape due to the physical limitations of the apparatus. Even once the event is written on to tape it can still be "lost" if it does not meet the selection criteria in the reconstruction program. These combined losses define the acceptance of the experiment which is determined by a Monte Carlo.

The Monte Carlo is a computer simulation of the experiment with a randomly generated sample of decay particles whose momenta and decay vertices are determined by their decay equation. For kaons this decay equation is not a pure exponential defined by the particle's lifetime, but includes interference terms due to CP symmetry violation as shown in equation 1.56. For each Monte Carlo event, simulated detector signals were generated. All Monte Carlo events were reconstructed in the same manner as the real data events in order to accurately reflect any biases due to reconstruction and selection criteria.

The Monte Carlo was set up to simulate all relevant particle decays. The same Monte Carlo was used to simulate $\mathrm{K} \rightarrow 2 \pi$ and $\mathrm{K} \rightarrow 3 \pi$ decays. For $\mathrm{K} \rightarrow 3 \pi$ decays several extra subroutines were called to simulate $\pi^{0}$ decay and added trigger elements. Events were generated with a momentum and neutral beam phase space distributions to match those found in the real data events. The apertures of the collimator and spectrometer were simulated in the Monte Carlo. The effects of the magnetic fields and multiple coulomb scattering on the charged particle trajectories were simulated also. Detailed analysis of the data provided noise rates for the chambers, hodoscopes and triggers. These were then used in the Monte Carlo to simulate the spectrometer performances more precisely. The $\mathrm{e}^{+} \mathrm{e}^{-}$data was invaluable in simulating the lead glass response in the Monte Carlo.

For Monte Carlo events which satisfied the simulated electronic triggers, event 
records as described in Section $\mathbf{5 . 4}$ were created and recorded. These records were identical in format to the original data and were read out and reconstructed in the same manner as the data.

\subsection{General Description}

\subsubsection{Data Bases}

The Monte Carlo read in an event description file which told it the particle decay tree, the production target, the momentum range and the $z$ vertex range. This information was read in once as it did not change from tape to tape. Another file gave the momentum spectrum to be used for the data set. Next the Monte Carlo read in a file which told it how many data tapes to simulate and what tape numbers they were. Some efficiencies and noise rates were kept constant for the entire data sample while others varied form tape from tape. These are listed in Tables 7.1 and 7.2 and will be explained later, except for (1) and (2) which were described in 6.2.3.

1 Number of triggers on the corresponding data tape

$2 x, y$ of the beam on the targets (see Section 6.2.2)

$3 x, y$ of the chamber centers (although they generally did not vary) (see Section 6.2.1)

4 lead glass calibration constants for each block (see Section 6.4)

5 MLU Trigger Processor first level efficiency (see Section 4.3.4)

6 CMR Trigger Processor first level efficiency (see Section 4.4.4)

7 MWPC efficiencies and dead cards (see Section 3.2.1)

8 TDC average times for each lead glass block (see Section 7.6) 


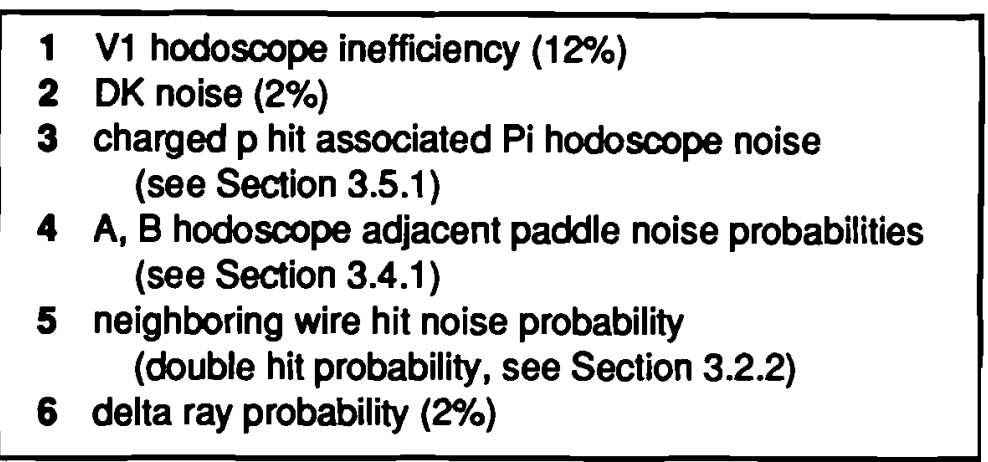

Table 7.2 Values Kopt Constant over the Data Sample

\subsubsection{Neutral Particle Decay}

After this information was read in the event generation loop started. The main program called the decay subroutine which simulated the particle decay. In order to speed up the Monte Carlo program, lookup tables were made to determine realistic momentum values for the decay. The reconstructed data momentum spectrum determined the input Monte Carlo momentum spectrum by requiring the reconstructed Monte Carlo momentum spectrum distribution to match that of the data.

For every $10 \mathrm{GeV}$ in momentum a lifetime weighted probability of decay was calculated and weighted by the input momentum spectrum. Equations 9.1 and 9.2 define the probability of decay for a $\mathrm{K} \rightarrow 3 \pi$ over a defined mometum $(\mathrm{p})$ and decay (z) range. Equations 9.15 and 9.16 define the probability for $\mathrm{K} \rightarrow 2 \pi$ decay. For every $10 \mathrm{GeV}$ increment in momentum, equation 9.1 (9.16) was integrated from the downstream end of the decay region to the target to determine the probablility of decay. The table of momentum versus probablility was normalized to one and used to determine the kaon momentum distribution. A random number from 0 to 1 would index a given $10 \mathrm{GeV}$ momentum bin. The momentum was interpolated over the 10 $\mathrm{GeV}$ bin using the random index value. The $\mathrm{z}$ of the decay was also determined from a weighted normalized $\mathrm{z}$ distribution, but was calculated for each event after determining the momentum of the decay, using equations 9.1 (9.16) and 9.2. The $\mathrm{K} \rightarrow 2 \pi$ momentum and vertex distributions were determined with $\eta_{+-}=0.00227$ and $\varphi_{+-}=46.4^{\circ(39)}$, where as the $\mathrm{K} \rightarrow 3 \pi$ momentum and vertex distributions were determined with $\eta_{+-0}=0.0$. 
For both decays the origin and slope of the particle with respect to the target was calculated in the following manner. A random point (from a Gaussian distribution) was chosen on the target and in the defining section of the collimator. A new random target point was tried if the particle did not project through the hole at the downstream end of the collimator. Once this was achieved, the momentum, position and $z$ of the decay were known. The kaon was then decayed into two charged pions or two charged pions and a neutral pion which immediately decayed into two photons. For $\mathrm{K} \rightarrow 3 \pi$ decay the program checked to see if the photons made it through the magnet aperature and hit the lead glass array. From all this information the program generated the chamber, hodoscope and, for $\mathrm{K} \rightarrow 3 \pi$ decay, lead glass block hits. A multiple scattering routine was called whenever a charged particle passed through material on its way through the spectrometer to simulate multiple coulomb scattering.

\subsubsection{The Trigger}

Once all the the hits had been generated, the Monte Carlo checked to see that the event passed the various trigger requirements. For $K \rightarrow 2 \pi$ decays the events had to pass the MLU simulation program which included $A$ and $B$ hodoscope noise and inefficiencies and MLU inefficiencies. The $K \rightarrow 3 \pi$ decays had to pass the MLU and, in addition, the CMR Trigger Processor simulation programs. CMR inefficiencies as described in Section 4.4.4 were included in this routine. A glass routine, which will be described in detail later in Section 7.6, generated ADC and TDC counts for all the lead glass blocks. This lead glass information was then used to determine if the event passed the GCF Trigger Processor simulation program. If the event passed all the $\mathrm{K}_{3 \pi} / \mathrm{K}_{2 \pi}$ trigger requirements it was then written out to a simulated raw data tape.

\subsection{Multiple Coulomb Scattering and Double Hits}

In Appendix $B$ is a list of all the material in our spectrometer and the number of radiation lengths in each piece. The total number of radiation lengths in our experiment was 0.1075 if the particle passes through the overlap region of both hodoscope arrays or $\mathbf{0 . 0 7 5 1}$ if it passes through one $A$ and one $B$ hodoscope element. The $A$ and $B$ hodoscopes were the largest contribution to the multiple scattering.

Multiple coulomb scattering was simulated using the Particle Data Group 40 pion multiple scattering formula for small angles, and the two uncorrelated 
equations for the $\mathrm{y}$ plane and $\theta$ plane as shown in Figure 7.1 and equation 7.1. $\mathrm{X}$ and y scattering was done independently.

$$
\theta_{o}=\frac{14.1 M e V / c}{p \beta} Z_{\text {inc }} \sqrt{L / L_{R}}\left[1+\frac{1}{9} \log _{10}\left(L / L_{R}\right)\right] \text { radians }
$$

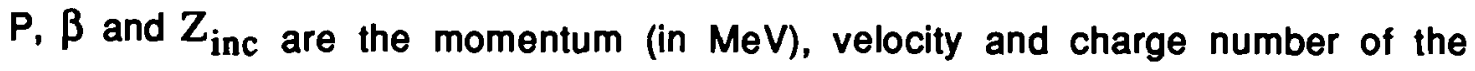
incident particle. $L / L_{R}$ is the thickness in radiation lengths of the scattering medium. The angle, $\theta_{0}$, is a fit to Moliere theory, accurate to $\sim 5 \%$ for $10^{-3}<\mathrm{L} / \mathrm{L}_{\mathrm{R}}$ $<10$ except for very light elements or low velocity.

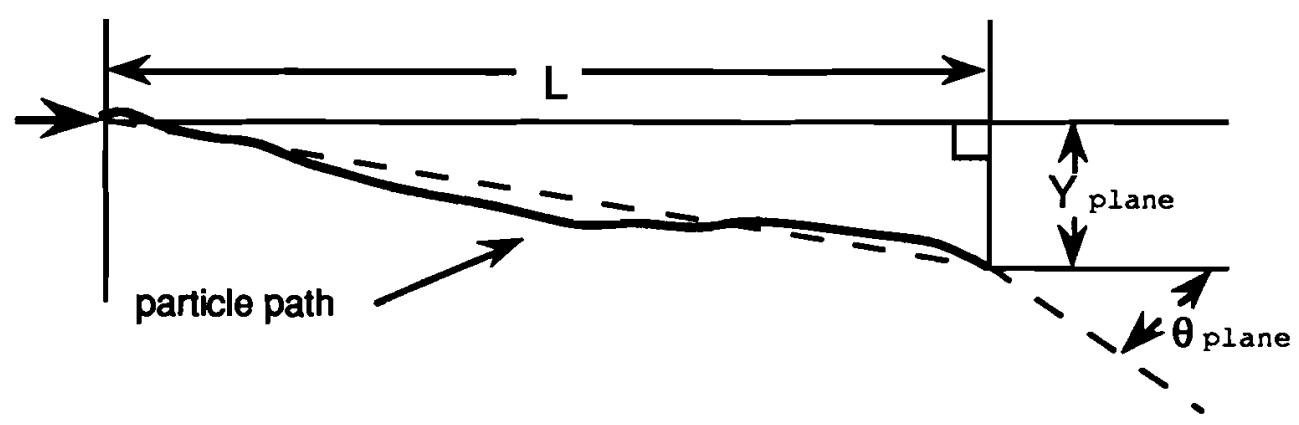

Flgure 7.1 Multlple Scattering Parameters

For a Monte Carlo it is convenient to work with independent Gaussian random variables, $\left(z_{1}, z_{2}\right)$, with mean zero and variance one.

$$
\begin{gathered}
\theta_{\text {plane }}=z_{2} \theta_{o} \\
y_{\text {plane }}=z_{1} L \theta_{o} / \sqrt{12}+z_{2} L \theta_{o} / \sqrt{2}
\end{gathered}
$$

where $\theta_{0}$ is defined by equation 7.1.

The multiple scattering parameters in the Monte Carlo simulation were verified by graphing $\sigma^{2}$ versus $1 / p^{2}$ where $\sigma$ is the width of a chamber offset and $p$ is the momentum of the charged track used in determining the offset. The offset is of the form:

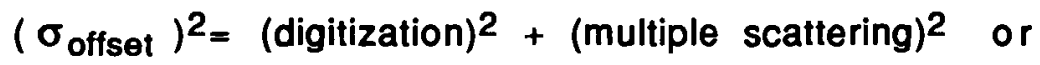

$$
\begin{aligned}
& \left(\sigma_{\text {offset }}\right)^{2}=\mathrm{D}+\mathrm{K} / \mathrm{p}^{2}
\end{aligned}
$$

This equation can be deduced from the PDG equation: $\quad y_{\text {plane }}^{r m s}=\frac{1}{\sqrt{3}} L \theta_{o}$ 


\section{8}

and equation 7.1 above for particles of the same mass and charge passing through the same apparatus. The slope of $\sigma^{2}$ versus $1 / \mathrm{p}^{2}$ is due to the multiple scattering since as $p$ increases the multiple scattering will decrease causing $\sigma$ to decrease. The intercept, $\mathrm{D}$, is given by the digitization of the wires which does not change with momentum. If a charged particle passes between two wires in a given chamber it has a certain likelihood of registering on both wires. This is the double hit probablility which is the major contribution to digitization. The double hit probability was calculated for each plane in each chamber. It appeared constant from tape to tape within errors. Thus the Monte Carlo used only one set of double hit probability numbers for the entire run. The values of the double hit probabilities used in the Monte Carlo are shown in Table 3.3 column 2.
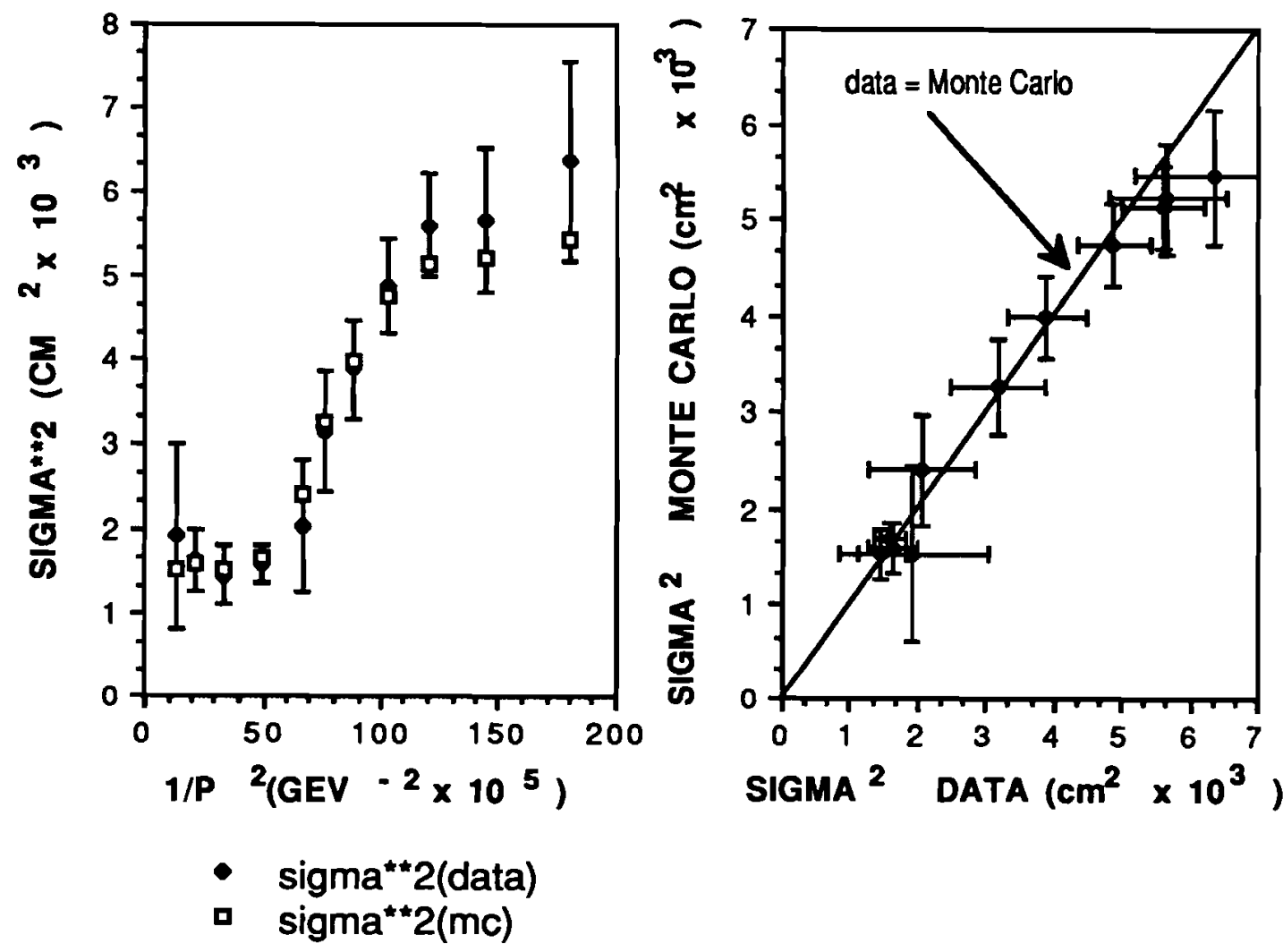

Flgure $7.2 \sigma^{2}$ ve. $1 / p^{2}$

Figure 7.3 $\sigma^{2}$ Data vs. $\sigma^{2}$ Monte Carlo 
Once the multiple scattering and double hit probabilities were in the Monte Carlo, a calculation of $\sigma^{2}$ of C5y versus $1 / \mathrm{p}^{2}$ for both the Monte Carlo and the data verified the simulation routine (Figure 7.2). The multiple scattering is minimal in this experiment so one must use a downstream chamber to see any effect at all.

Chamber offset widths were smaller in the Monte Carlo even after adding multiple scattering to the program and matching the double-hit percentages between data and Monte Carlo. This indicated that the double-hit probability was too high in the Monte Carlo. The surplus 2-wire clusters in the data were attributed to random processes such as delta rays (low energy electrons). The delta rays were simulated by generating $2 \%$ of the chamber track hits in each plane with random hits in adjacent wires. This improved the agreement between data and Monte Carlo of the $\sigma^{2}$ versus $1 / \mathrm{p}^{2}$ intercept, the chamber residuals and the chi-squared per degree of freedom of the charged track fit while keeping the agreement between data and Monte Carlo of the double-hit percentages.

\subsection{Fringe Fields}

Section 6.3.1 discussed the measurement of the analysis magnets' fringe fields. There was no measureable effect of fringe fields in either the $\mathrm{K} \rightarrow 2 \pi$ or the $\mathrm{K} \rightarrow 3 \pi$ chamber offsets. The Monte Carlo program did not include a fringe field in its calculation of the charged track projections.

\subsection{Efficiencies and Noise}

Efficiencies and noise rates for the various parts of the spectrometer are covered in Chapters 3 and 4 . Table 7.3 shows which elements' efficiencies or noise rates (accidentals) were included in the Monte Carlo program. 


\begin{tabular}{|c|c|c|c|}
\hline Element & Inefficiencies & Noise & Comments \\
\hline V1 Hodoscope & $\begin{array}{c}\text { one for the } \\
\text { data set- } 12 \%\end{array}$ & no & \\
\hline DK Hodoscope & no & $\begin{array}{c}\text { one for the } \\
\text { data set - 2\% }\end{array}$ & \\
\hline MWPC's & tape by tape & no & $\begin{array}{c}\text { dead cards } \\
\text { tape by tape }\end{array}$ \\
\hline $\begin{array}{c}\text { A,B Hodoscopes } \\
\text { tape by tape }\end{array}$ & see comment & $\begin{array}{c}\text { noise folded into } \\
\text { efficiencies }\end{array}$ \\
\hline $\begin{array}{c}\text { CMR Trigger } \\
\text { Processor }\end{array}$ & tape by tape & no & \\
\hline $\begin{array}{c}\text { MLU Trigger } \\
\text { Processor }\end{array}$ & tape by tape & no & \\
\hline \begin{tabular}{c} 
Pi Hodoscopes \\
\hline
\end{tabular} & no & one for the \\
data set & \\
\hline
\end{tabular}

Table 7.3 Monte Carlo Nolses and Efflclencies

\subsection{The Shower Monte Carlo}

\subsubsection{Introduction}

The shower Monte Carlo starts with the $\mathrm{x}$ and $\mathrm{y}$ position and energy of each photon determined from the $\pi^{\circ}$ decay from the parent kaon. Each photon is checked to see if it vetoes itself through the GCF trigger processor by backsplashing in the $\mathrm{Pi}$ hodoscopes. For the region of the lead glass array that was behind the Pi hodoscopes this probablility was between 10 and $30 \%$ (see Section 3.5.1, Figure 3.6). The energy in the lead glass array is distributed using an error function distribution centered at the calculated photon hit position.

There were two major sources of energy losses. For energy lost out the back of the array, a linear correction shifted the energy of the shower to account for the loss. Losses through the cracks between the blocks and through the hole in the center of the array were simulated using $e^{+} e^{-}$data distributions. The final energy was then converted into ADC counts. 
A file of inactive blocks for each tape ensured that none of these blocks had signals in them in the Monte Carlo. The file of average TDC times from the data was used to obtain the TDC times. The program then wrote out the ADC counts, the TDC times, the hit lead glass block numbers and the number of hit blocks.

\subsubsection{Shower Shapes}

The photon shower shape was simulated as a sum of two gaussians. Different shower shapes were used depending on where the photon showered in the lead glass array. Showers within $5 \mathrm{~cm}$ of the hole in the array (hereafter designated hole showers) were significantly larger than non-hole showers due to the fact that the hole showers are higher in energy and are missing a neighboring block on one side. Similarly showers near the edge of a block were larger due to the cracks between the blocks. Comparisons between $\mathrm{K}_{3 \pi}$ Monte Carlo and data for the number of blocks in a shower determined the shower parameters for the two gaussians for the different categories. After matching the overall shower shapes, the Monte Carlo and data energy dependence of the shower radius matched well (Figure 7.4).

Holes

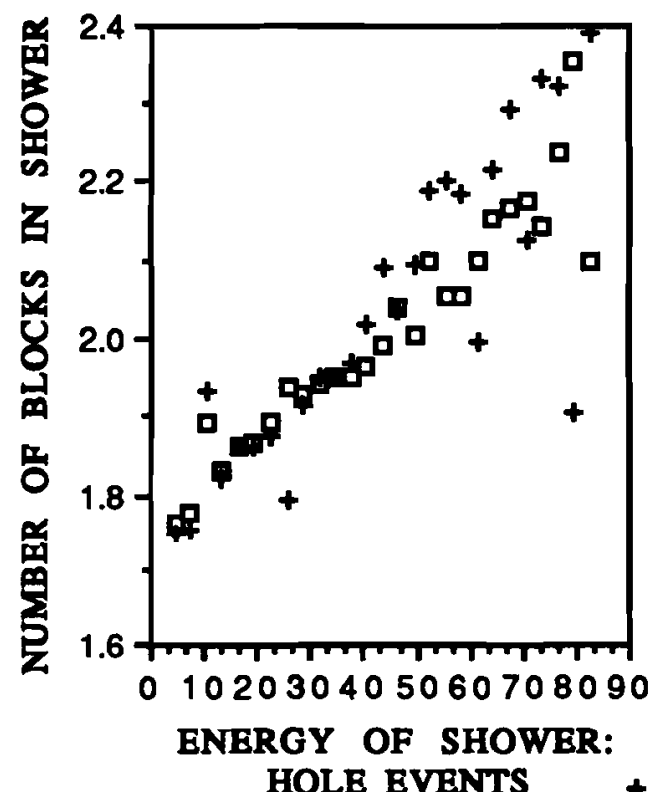

(GEV)

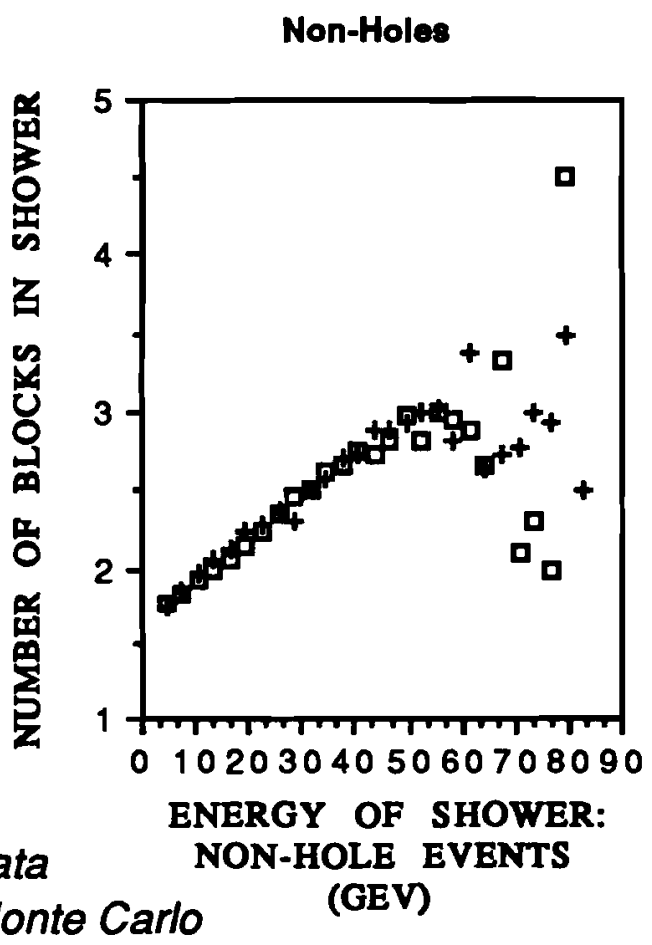

- Monte Carlo

Flgure 7.4 Radlus ve. Energy for Data and Monte Carlo 


\subsubsection{Energy Losses}

There were two major sources of energy losses of photons in the lead glass array. Higher energy gammas lost energy out the back of the lead glass array. The other source of energy loss, through the cracks between the blocks or the hole in the center of the array did not effect centrally located showers. These energy losses were studied using $e^{+} e^{-}$data and plotting E/P in terms of $x y$ area. Xy area is the product of the distance from the projected track location at the glass array to its nearest block $x$ edge and $y$ edge normalized to $1 / 4$ of a block area:

$$
\mathrm{xy} \text { area }=\frac{\left\{\left|x_{\text {hit }}-x_{\text {block edge }}\right| \times\left|y_{\text {hit }}-y_{\text {block edge }}\right|\right\}}{\left\{\frac{1}{4}\left\{x_{\text {width }} \times y_{\text {width }}\right\}\right\}}
$$

This defines $x y$ area $=0$ as a hit on the edge of a block and $x y$ area $=1.0$ as a hit in the block's center. $\mathrm{E}$ is the energy of the shower and $\mathrm{P}$ is the momentum of the electron/positron track associated with the shower.

From graphs of E/P vs. $x y$ area for various energies it appeared that crack and hole energy losses became important for $x y$ area $<0.3$. Because of this central hits were defined as $x y$ area $>0.3$, Looking at these events in terms of E/P determined the shower energy as a function of track momentum needed to account for energy losses out the back of the array. $\mathrm{E}$ as a function of $\mathrm{P}$ is shown in Figures 7.5 and 7.6 for both non-hole and hole hits. The line drawn corresponds to a fit to the $e^{+} e^{-}$data points shown in the plots.

These "corrections" applied to the $e^{+} e^{-}$data shifted the E/P of the showers. Figure 7.7 shows the uncorrected and corrected $\mathrm{E} / \mathrm{P}$ plots for showers near the hole. The vertical line corresponds to $\mathrm{E}=\mathrm{P}$ or $\mathrm{E} / \mathrm{P}=1$, the desired situation. For showers more than a block from the hole the corrections were very small and both the corrected and uncorrected E/P plots peaked very near 1.0.

Now a simulation of the energy resolution smearing due to crack and hole losses was needed. Files of E/P plots for every 0.05 in $x y$ area were created from the $\mathrm{e}^{+} e^{-}$ data after shifting the $e^{+} e^{-}$data according to the linear functions $E(P)$. These plots contained all the information needed to simulate the crack and hole losses. The plots were done for non-hole and hole data seperately since the energy resolution was 


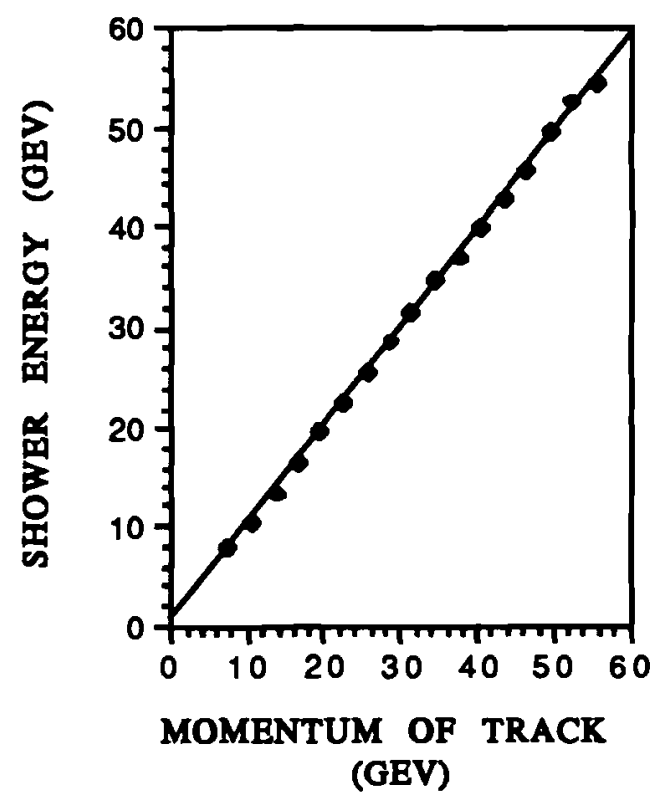

Flgure 7.5 P vs. E Non-hole Showers

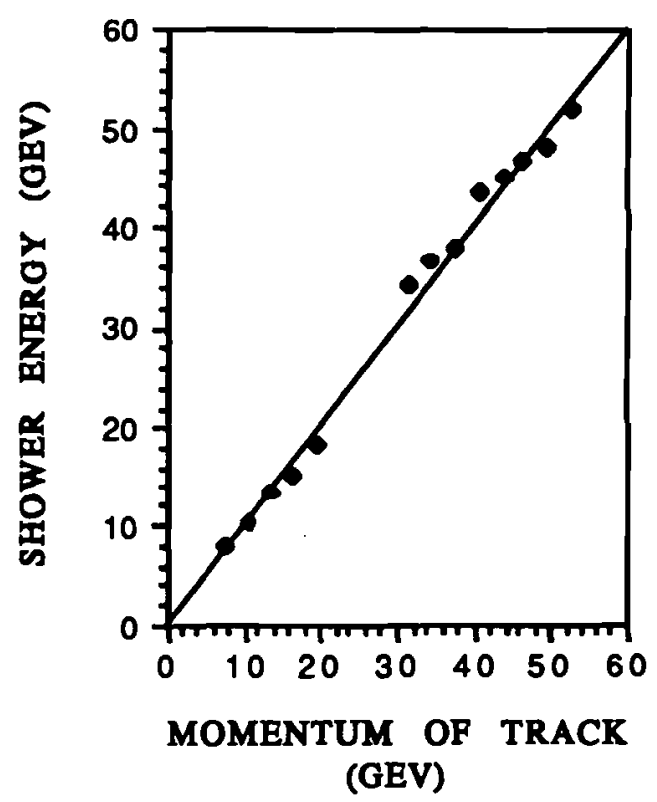

Flgure 7.6 P vs. E Hole Showers

\section{Curve fits:}

For non-hole showers: $\quad E=0.518+0.980 \cdot P$

For hole showers: $\quad E=0.069+1.018 * P$

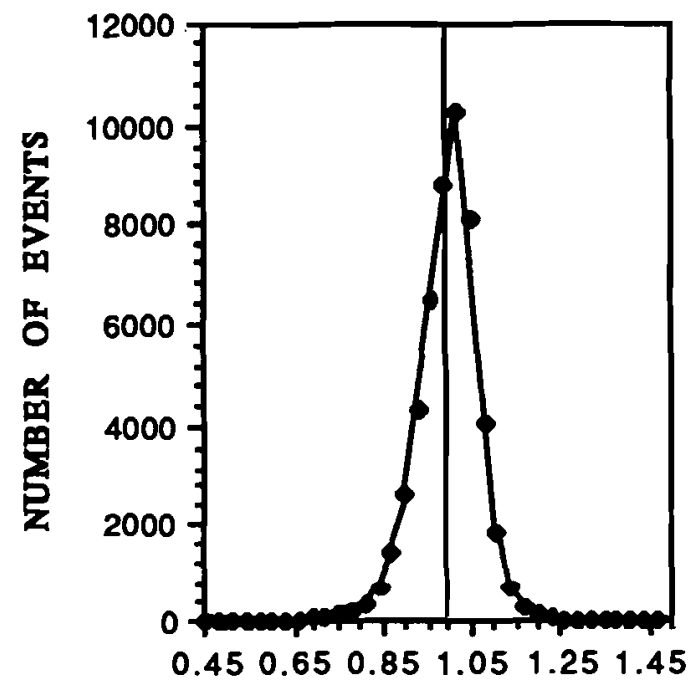

SHOWER ENERGY TRACK MOMENTUM

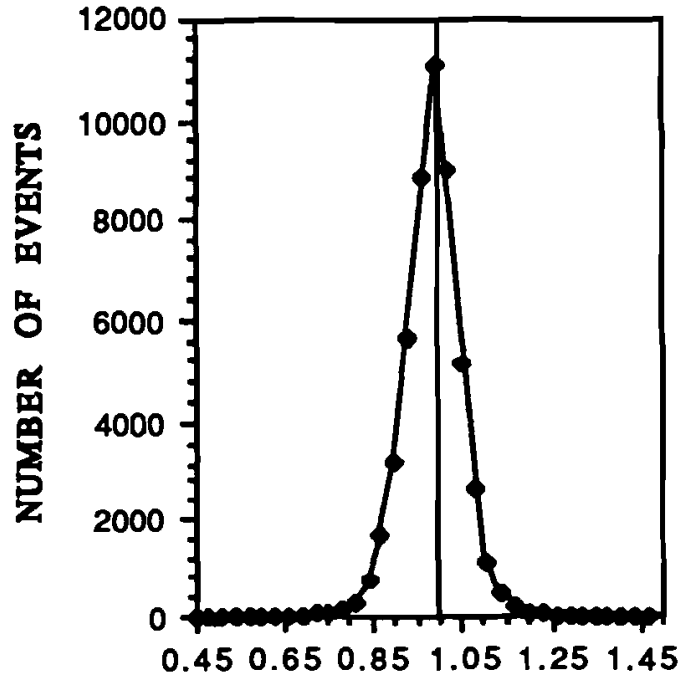

CORRECTED SHOWER ENERGY

Flgure 7.7 E/P for $e^{+} e^{-}$for Hole Showers (actual and corrected) 
different for the two different categories. The $\mathrm{K}_{3 \pi}$ Monte Carlo then adjusted the photon shower energies according to these distributions.

Events within $2.54 \mathrm{~cm}$ of the hole were not treated in the aforementioned manner because their energy resolution was worse due to their proximity to the hole. They were shifted according to energy losses out the back of the array as for the other showers. For the final E/P resolution distributions, the inner area around the hole was divided into 18 regions as shown in Figure 7.8 to more accurately simulate the changes in resolution as photons shower closer to the hole. The peak of the E/P distributions shifted lower and the width increased for regions closer to the center of the hole. For each of the 18 regions an E/P distribution was recorded from the $e^{+} e^{-}$data and the Monte Carlo simulated the energy losses using these distributions.

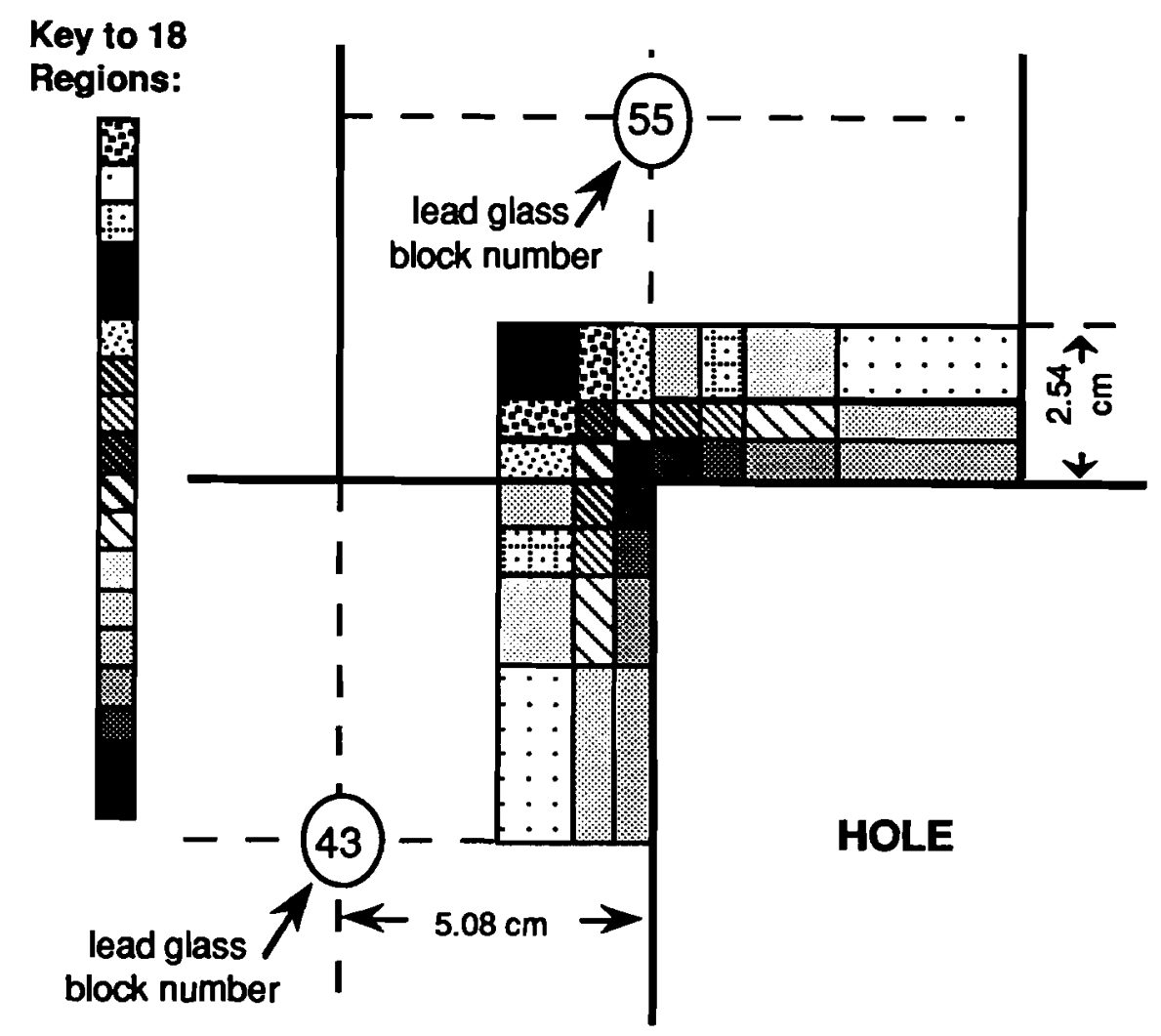

Figure 7.8 Hole Inner Regions 


\subsubsection{TDC Thresholds}

GCF Trigger Processor TDC and regular TDC thresholds were simulated in the Monte Carlo by error function distributions which were determined by fitting the data distributions. The Monte Carlo and data distributions matched very well using these error function distributions. This ensured that the Monte Carlo accurately simulated the loss of lead glass block signals near threshold. 


\section{Chapter Eight}

\section{Data Selection}

\subsection{Introduction}

This analysis started with 2.03 million $K_{2 \pi}$ triggers and 9.36 million $K_{3 \pi}$ triggers. Obtaining a pure sample of events required finding variables where Monte Carlo and data discrepancies occurred. If the discrepancy was understood and corresponded to poorly reconstructed or non-kaon events, one could determine selection criteria based on the variable and reduce the number of undesired events in the sample. The difficulty lies in trying to obtain a pure kaon sample while trying to keep introduced biases to a minimum.

The foundation for the selection criteria for the $K_{2 \pi}$ and $K_{3 \pi}$ samples is

discussed first. Section 8.3 covers the common $K_{2 \pi}, K_{3 \pi}$ selection criteria and Section 8.4 continues with the additional $K_{3 \pi}$ selection requirements.

All comparison plots will be shown for west beam downstream data unless otherwise specified. Data events are shown as the fitted histogram when a fit is shown, and Monte Carlo events are shown as points only.

\subsection{Signals and Background}

There were three data types of interest in my method of measuring $\eta_{+-0}$. The main data sample consisted of $\mathrm{K}_{3 \pi}$ triggers which could be reconstructed to $\mathrm{K}^{\mathbf{0}} \rightarrow$ $\pi^{+} \pi^{-} \pi^{\circ}$ candidates. The other two samples were $K_{2 \pi}$ triggers which could be reconstructed to $\mathrm{K}^{0} \rightarrow \pi^{+} \pi^{-}$decays and all triggers whose charged tracks reconstructed to a vertex. The $K_{2 \pi}$ sample was needed to measure the normalization of the number of kaons produced in the target, a value used to constrain the $\eta_{+-0}$ measurement. This sample also provided a check for the charged track portion of the $\mathrm{K}^{0} \rightarrow \pi^{+} \pi^{-} \pi^{0}$ analysis. The third sample of various particles decays from many triggers provided the information needed to determine the efficiencies of the 
apparatus which were then used in the Monte Carlo program. The various backgrounds present in the $\mathrm{K}_{2 \pi}$ and $\mathrm{K}_{3 \pi}$ trigger samples needed to be reduced as much as possible in order to determine the normalization and $\eta_{+-0}$ as accurately as possible.

\subsection{1 $\mathrm{K}_{3 \pi}$ Sample}

The main sources of background in the $K_{3 \pi}$ sample were $K^{0} \rightarrow 2 \pi$ and $\Lambda^{0} \rightarrow p \pi$ decays with accidental photons and $\Xi^{0} \rightarrow \Lambda \pi^{0}$ since there were nearly equal numbers of lambdas and kaons in the neutral beam. $95 \%$ of the lambda candidates were removed by the CMR and MLU trigger processors. Only $10 \%$ of the $\mathrm{K}_{3 \pi}$ triggers reconstructed to a lambda candidate and these were removed by reconstructing the charged mass as a proton and a pion and cutting out events with a mass within $10 \mathrm{MeV}$ of the lambda mass. The other background, $\mathrm{K}^{0} \rightarrow 2 \pi$ decays, accounted for $50 \%$ of the $\mathrm{K}_{3 \pi}$ triggers. The GCF trigger processor reduced these events by requiring two neutral showers in the lead glass array. Since it only considered nearest neighbor blocks as a shower, large charged pion showers were sometimes identified as two showers, one charged and one neutral. If both charged pions showered in the glass, two extra neutral showers were sometimes found and thus a $\mathrm{K}^{\circ} \rightarrow 2 \pi$ decay would be accepted in the $\mathrm{K}_{3 \pi}$ trigger. These $\mathrm{K}^{0} \rightarrow 2 \pi$ candidates were easily removed from the $\mathrm{K}^{0} \rightarrow 3 \pi$ sample by eliminating events with a $\pi^{+} \pi^{-}$mass within $25 \mathrm{MeV}$ of the kaon mass. Also the showers associated with actual $\mathrm{K}^{0} \rightarrow 2 \pi$ and $\Lambda^{0} \rightarrow \mathrm{p} \pi$ decays would not reconstruct to a good $\pi^{\circ}$ mass and were removed by requiring the $\pi^{\circ}$ mass be between 70 and $210 \mathrm{MeV}$.

\subsection{2 $\mathrm{K}_{2 \pi}$ Sample}

The $K_{2 \pi}$ sample of events was used to cross-check the charged track portion of the $\mathrm{K}_{3 \pi}$ Monte Carlo and analysis programs. The $\mathrm{K}^{\mathbf{0}} \rightarrow \pi^{+} \pi^{-}$data sample also determined the number of kaons produced on each target. Since the kaons are produced by the strong interaction, the number of $\mathrm{K}_{\mathrm{S}} \sim \mathrm{K}_{1}$ or $\left(\mathrm{K}^{\mathrm{O}}+\overline{\mathrm{K}^{\mathrm{O}}}\right)$ produced is the same as the number of $\mathrm{K}_{\ell} \sim \mathrm{K}_{2}$ or $\left(\mathrm{K}^{0}-\overline{\mathrm{K}^{0}}\right)$, and thus the normalization for the $\mathrm{K}^{\circ} \rightarrow 3 \pi$ sample can be taken from the $\mathrm{K}^{\circ} \rightarrow 2 \pi$ sample for which all the decay quantities are known.

The main sources of non-K $\rightarrow 2 \pi$ background in the $K_{2 \pi}$ trigger were $K^{0} \rightarrow 3 \pi$ 
and $\Lambda^{0} \rightarrow \mathrm{p} \pi$ decays. Typically $30 \%$ of the $\mathrm{K}^{0} \rightarrow 2 \pi$ triggers were possible lambda candidates and $4 \%$ were possible $\mathrm{K}^{\circ} \rightarrow 3 \pi$ candidates. Requiring that the $\pi^{+} \pi^{-}$mass be within $25 \mathrm{MeV}$ of the kaon mass and that the p $\pi$ mass not be within $10 \mathrm{MeV}$ of the lambda mass removed these $\Lambda^{0}$ and $\mathrm{K}^{0} \rightarrow 3 \pi$ candidates. Another source of background in the $\mathrm{K} \rightarrow 2 \pi$ data came from the residual proton beam hitting the collimator wall and producing particles. This source was significant only for the upstream west target when M1 had positive polarity. $\Lambda^{0}$ s and $K^{0} \rightarrow 3 \pi$ 's from this source could be removed with a $\pi^{+} \pi^{-}$mass constraint, but $\mathrm{K}^{0} \rightarrow 2 \pi$ decays could not. A $1.2 \%$ background had to be subtracted from the upstream west $K^{0} \rightarrow 2 \pi$ data sample in order to obtain the normalization factor to be used in the final $\mathrm{K}^{0} \rightarrow 3 \pi$ ratio fit. This same background was also present in the $K_{3 \pi}$ data sample, but was not enhanced by the $\mathrm{K}_{\mathfrak{\ell}} / \mathrm{K}_{\mathrm{s}}$ lifetime ratio. It was only $0.6 \%$ of the final upstream west $\mathrm{K}_{3 \pi}$ data. The methods used in removing these collimator produced kaons will be discussed in Section 8.3 and Appendix C.

\subsection{Common $\mathrm{K}_{2 \pi} / \mathrm{K}_{3 \pi}$ Data Requirements}

The charged track portion of the $\mathrm{K}_{2 \pi}$ and $\mathrm{K}_{3 \pi}$ triggers was similar, making data selection at that level identical. Both data samples neded to pass the slope ratio requirements of the MLU. The charged track reconstruction also needed to be good, as determined by the $\chi^{2} / D O F$ of the charged track fit, and the neutral kaon needed to point back to the target. These requirements comprised the common $K_{2 \pi}$ and $K_{3 \pi}$ data cuts.

\begin{tabular}{|l|c|c|}
\hline Variable & $\mathrm{K} \rightarrow 2 \pi$ Preliminary Cuts & $\mathrm{K} \rightarrow 3 \pi$ Preliminary Cuts \\
\hline $\mathrm{M}_{\pi+\pi}$ & $>0.425 \mathrm{GeV}$ & $>0.425 \mathrm{GeV}$ \\
$\chi_{\text {/DOF }}^{2}$ & $<3.0$ & $<3.0$ \\
$\mathrm{E}_{\gamma 1}$ & $\cdots$ & $>1.5 \mathrm{GeV}$ \\
$\mathrm{E}_{\gamma 2}$ & $\cdots$ & $>1.0 \mathrm{GeV}$ \\
\hline
\end{tabular}

Table 8.1 Preliminary $K_{2 \pi}$ and $K_{3 \pi}$ Data Selection 
Some data selection at the reconstruction level was discussed in Chapter 6. Table 8.1 shows the data selection criteria for $K_{2 \pi}$ and $K_{3 \pi}$ trigger events at the reconstruction level.

$E_{\gamma 1}$ and $E_{\gamma}$ is the energy of the higher energy and lower energy photon respectively. Events were also seperated on the basis of the $\mathrm{K}^{\circ}$ production target as defined by the projection of the beam at the collimator shown in Figure 6.25.

Both the $\mathrm{K}_{2 \pi}$ and the $\mathrm{K}_{3 \pi}$ triggers required the MLU Trigger Processor to identify symmetric charged tracks and thereby reduce the acceptance of $\Lambda^{0}$ decays. In the Monte Carlo, no noise hits were simulated in the $A$ and $B$ hodoscopes, the elements that the MLU Trigger Processor used to determine a slope ratio. Requiring that all events pass the simulation program of the MLU eliminated events which passed the MLU due to noise hits. Restricting the calculated slope ratio to be between 0.3 and 3.0, the values used as limits in both the MLU Trigger Processor and the CMR Trigger Processor, helped eliminate any lambda decays that may have been missed by the trigger processors. Data and Monte Carlo comparison plots are shown in Figure 8.1.

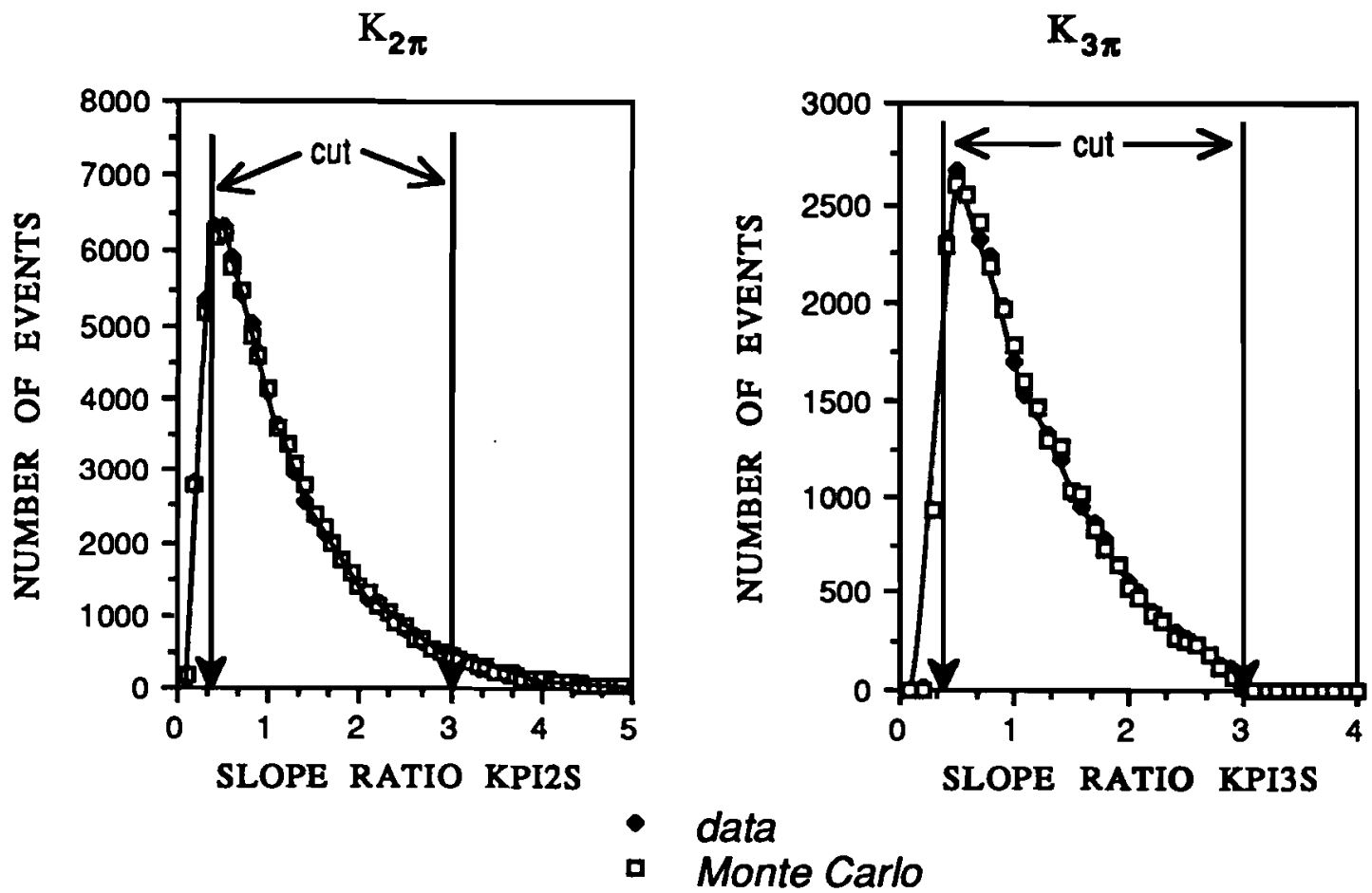

Figure 8.1 Slope Ratlo Requirement 
The $\chi^{2} /$ DOF of the charged track fit was tightened to less than 2.5 in order to remove events with too many hits in the chambers. I also required the maximum number of non-adjacent hits per plane in each chamber be four or less. Figure $\mathbf{8 . 2}$ shows the $\chi^{2}$ /DOF cut for $K_{2 \pi}$ and $K_{3 \pi}$ events on a logarithmic scale.

Poorly reconstructed and/or misidentified events and collimator produced kaons did not point back to the production target. The transverse momentum, $\mathbf{P}_{\mathrm{T}}$, was calculated by multiplying the reconstructed momentum of the parent particle with the angle of its momentum vector with respect to a line from the production target to the center of the collimator hole. A requirement that $\mathrm{P}_{\mathrm{T}}^{2}<0.033 \mathrm{GeV}^{2}$ removed these events. The $\mathrm{P}_{\mathrm{T}}{ }^{2}$ plot is shown in Figure 8.3 on a logarithmic scale. Note that the vertical axis for the $K_{2 \pi}$ and $K_{3 \pi}$ graphs do not have same scale.

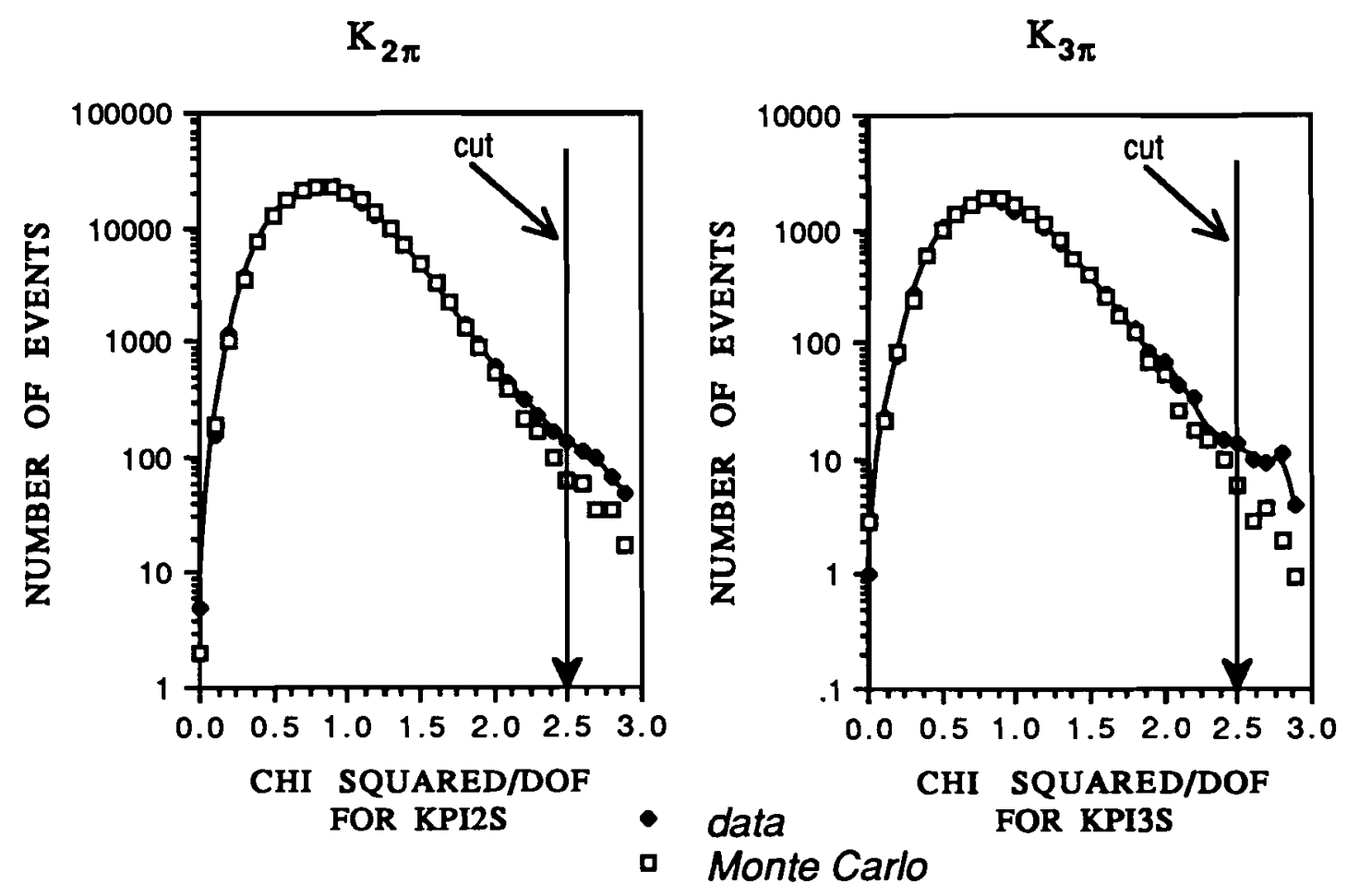

Flgure $8.2 \quad \chi^{2} / \mathrm{DOF}$ 


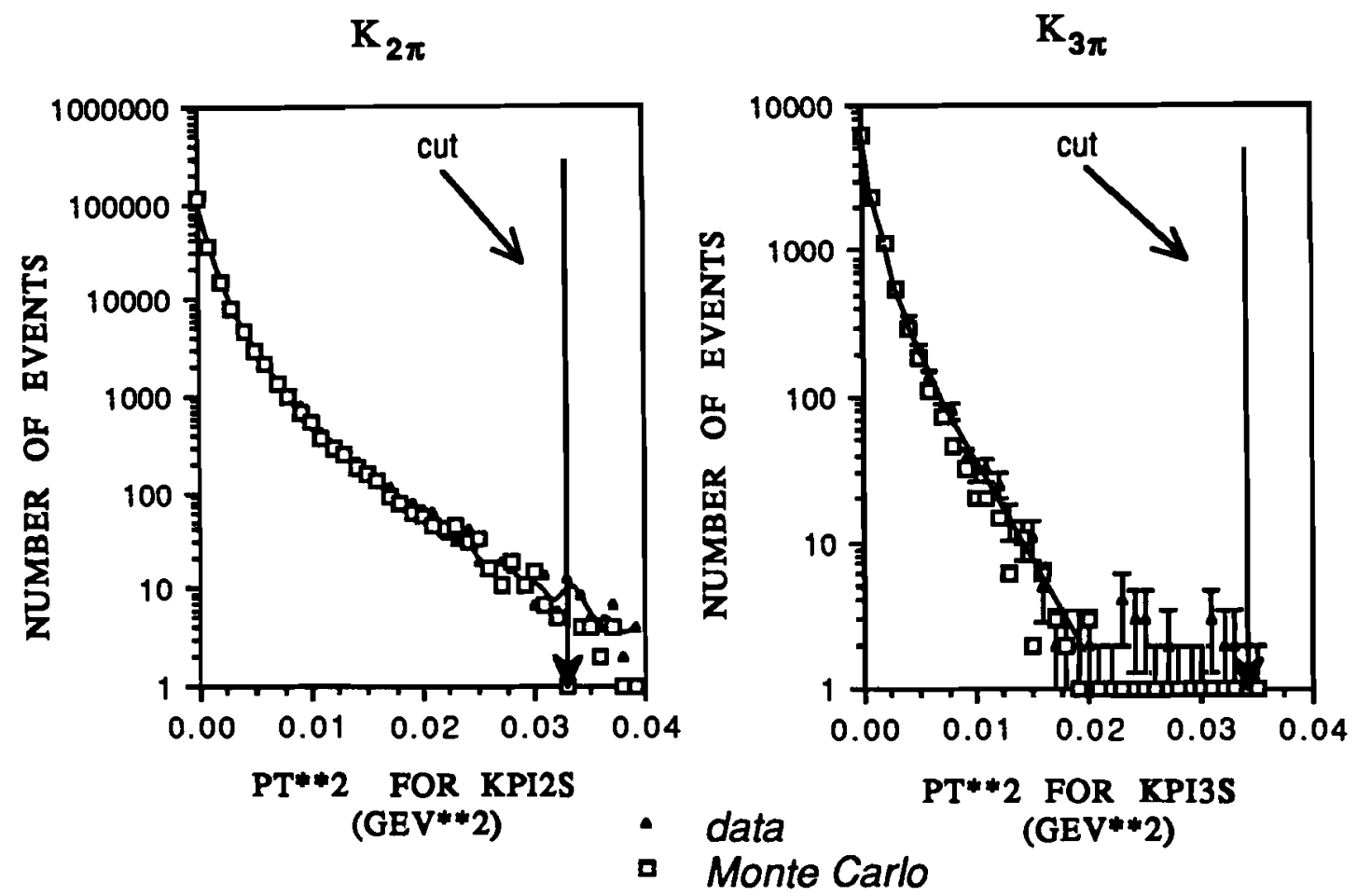

Figure 8.3 $\quad \mathbf{P}_{T^{2}}{ }^{2}$

To eliminated collimator production background and misidentified decays, the projected $\mathrm{K}^{\mathbf{0}}$ momentum was required to be close to the center of the target at $\mathrm{z}$ of the upstream target and close to the center of the glass at $z$ of the lead glass array. The maximum values $R^{2}$ ustgt and $R^{2}$ glass are given in Table 8.2. The beam angles $\Theta_{x}$ and $\Theta_{y}$, defined respectively as the momentum ratios $P_{x} / P_{z}$ and $P_{y} / P_{z}$ of the reconstructed kaon, defined a third beam parameter used to identify target produced kaons $\Theta=\sqrt{\Theta_{x}^{2}+\Theta_{y}^{2}}$. The maximum allowable values of $\Theta$ are also given in Table 8.2. Target-produced kaons had a narrow $\mathrm{R}^{2}$ distribution peaked near zero where as collimator produced events had a very broad distribution. This was true of the $\Theta^{2}$ distribution also.

A discussion of collimator produced backgrounds and the amount for various targets is given in Appendix C. Figures 8.4-8.9 show the upstream west data where collimator production background is largest. Both linear and logarithmic scales are shown in each figure. The upstream west data sample also has the worst agreement between Monte Carlo and data of beam phase space. Both the normalization of kaons 
on target from $\mathrm{K} \rightarrow 2 \pi$ decay and the measurement of $\eta_{+-0}$ from $\mathrm{K} \rightarrow 2 \pi$ decay were found to be insensitive to this discrepancy between the upstream west data and Monte Carlo (see Section 9.5.6).

\begin{tabular}{|c|c|c|c|c||c|c|c|c|}
\hline \multirow{2}{*}{ Variable } & \multicolumn{3}{|c||}{$K_{2 \pi}$ Beam Requirements } & \multicolumn{3}{c|}{$K_{3 \pi}$ Beam Requirements } \\
\cline { 2 - 9 } & DW & DE & UW & UE & DW & DE & UW & UE \\
\hline $\boldsymbol{\theta}$ & $<0.735$ & $<0.60$ & $<0.35$ & $<0.30$ & $<0.85$ & $<0.80$ & $<0.50$ & $<0.50$ \\
\hline $\mathbf{R}_{\text {ustgt }}$ & $<2.30$ & $<2.10$ & $<1.30$ & $<1.30$ & $<2.75$ & $<2.65$ & $<1.875$ & $<1.875$ \\
\hline $\mathbf{R}_{\text {glass }}$ & $<4.20$ & $<3.75$ & $<1.80$ & $<1.60$ & $<5.10$ & $<4.50$ & $<2.25$ & $<2.25$ \\
\hline
\end{tabular}

Table 8.2 Neutral Beam Phase Space Requirements
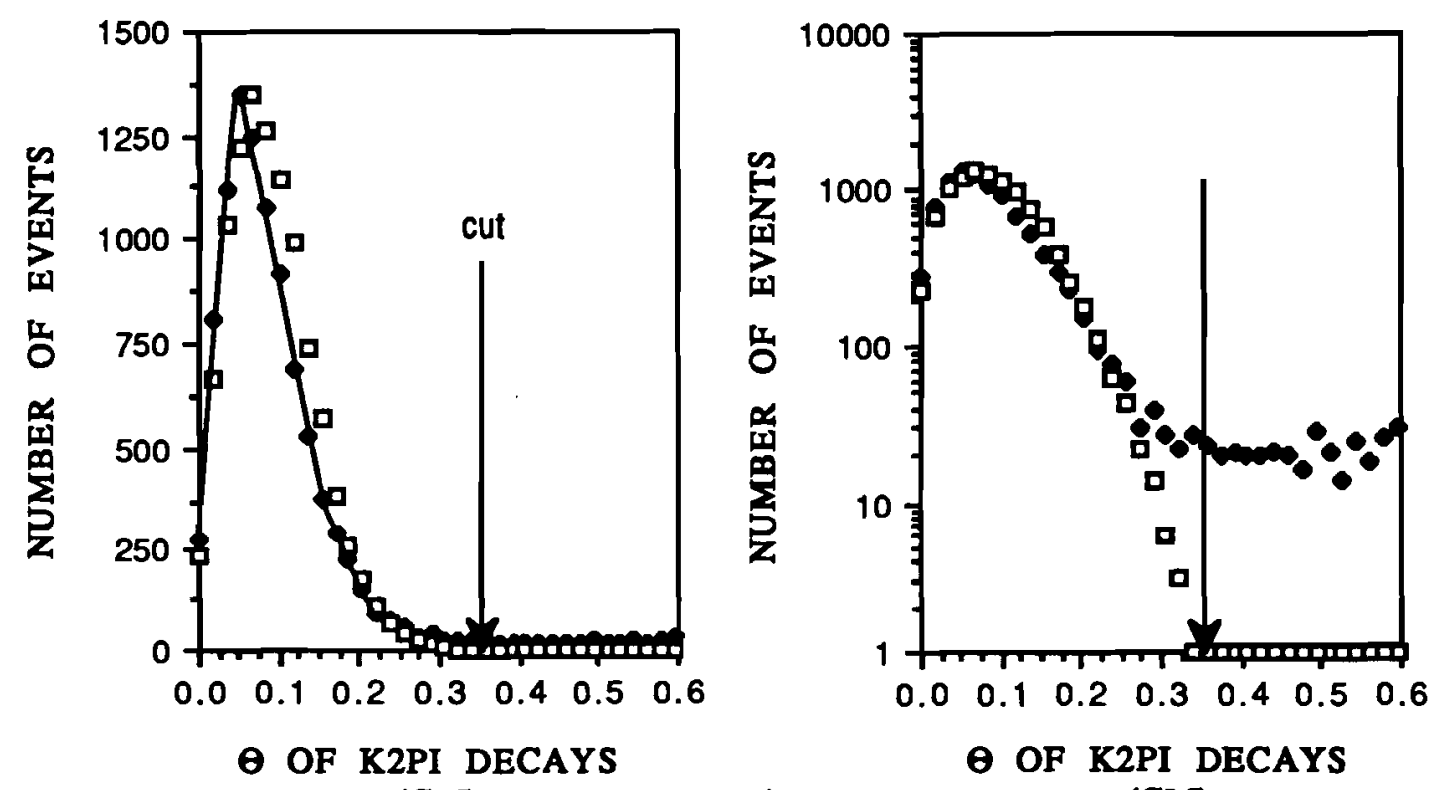

$\theta$ OF K2PI DECAYS

(CM)

- data

- Monte Carlo

(CM)

Figure 8.4 $\theta$ for $\mathrm{K} \rightarrow 2 \pi$ Events from the Upstream West Target 

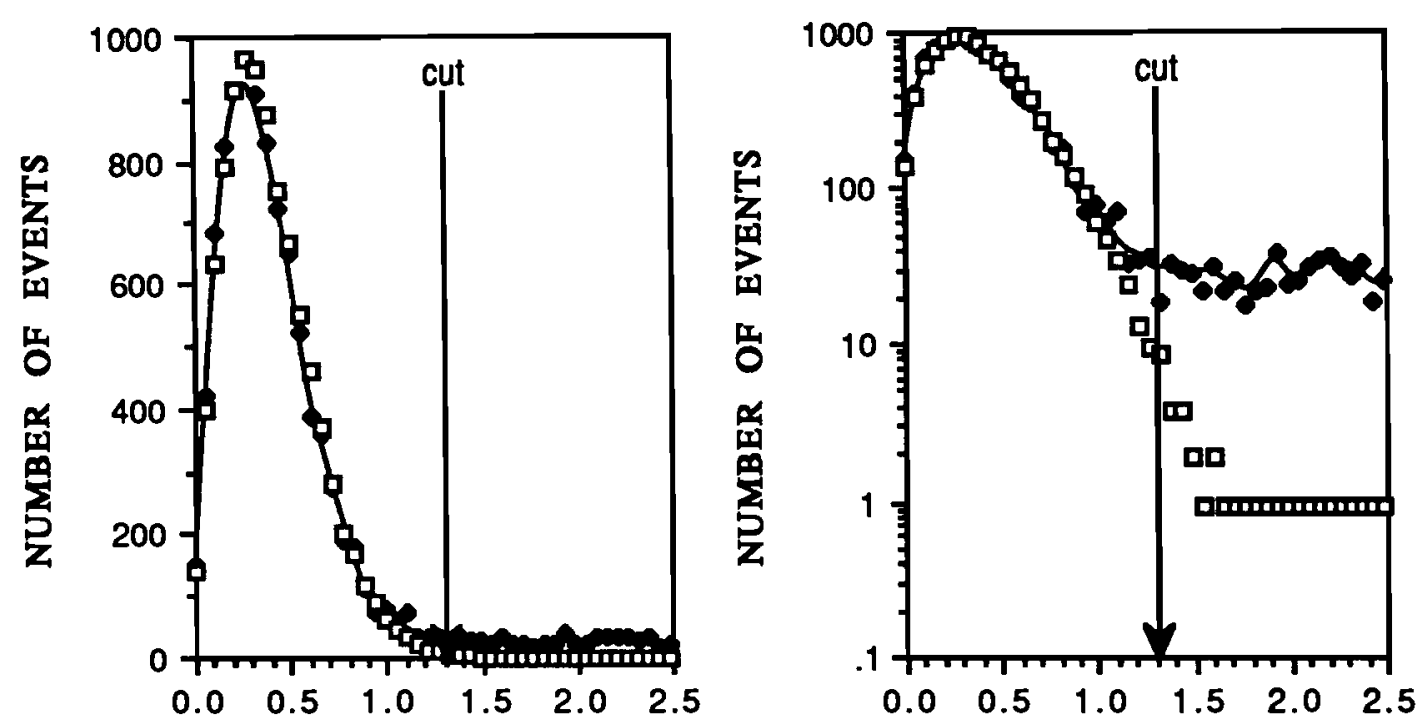

RADIUS AT UPSTREAM TARGET RADIUS AT UPSTREAM TARGET FOR K2PI DECAY (CM)

FOR K2PI DECAY

- Monte Carlo (CM)

Figure 8.5 Radius at the Upstream Target for $\mathrm{K} \rightarrow 2 \pi$ Events from the Upstream West Target

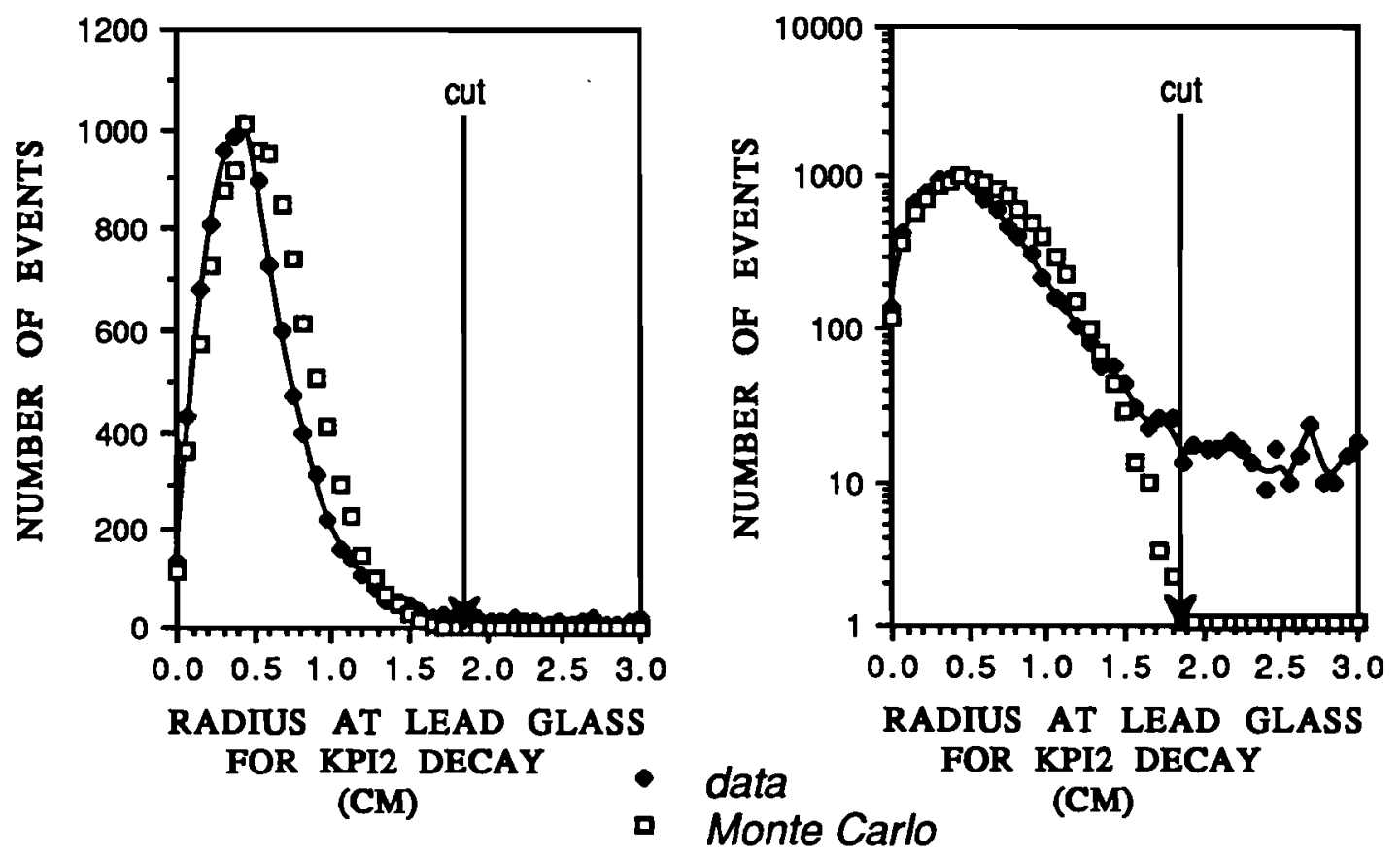

Flgure 8.6 Radius at the Glass for $\mathrm{K} \rightarrow 2 \pi$ Events from the Upstream West Target 

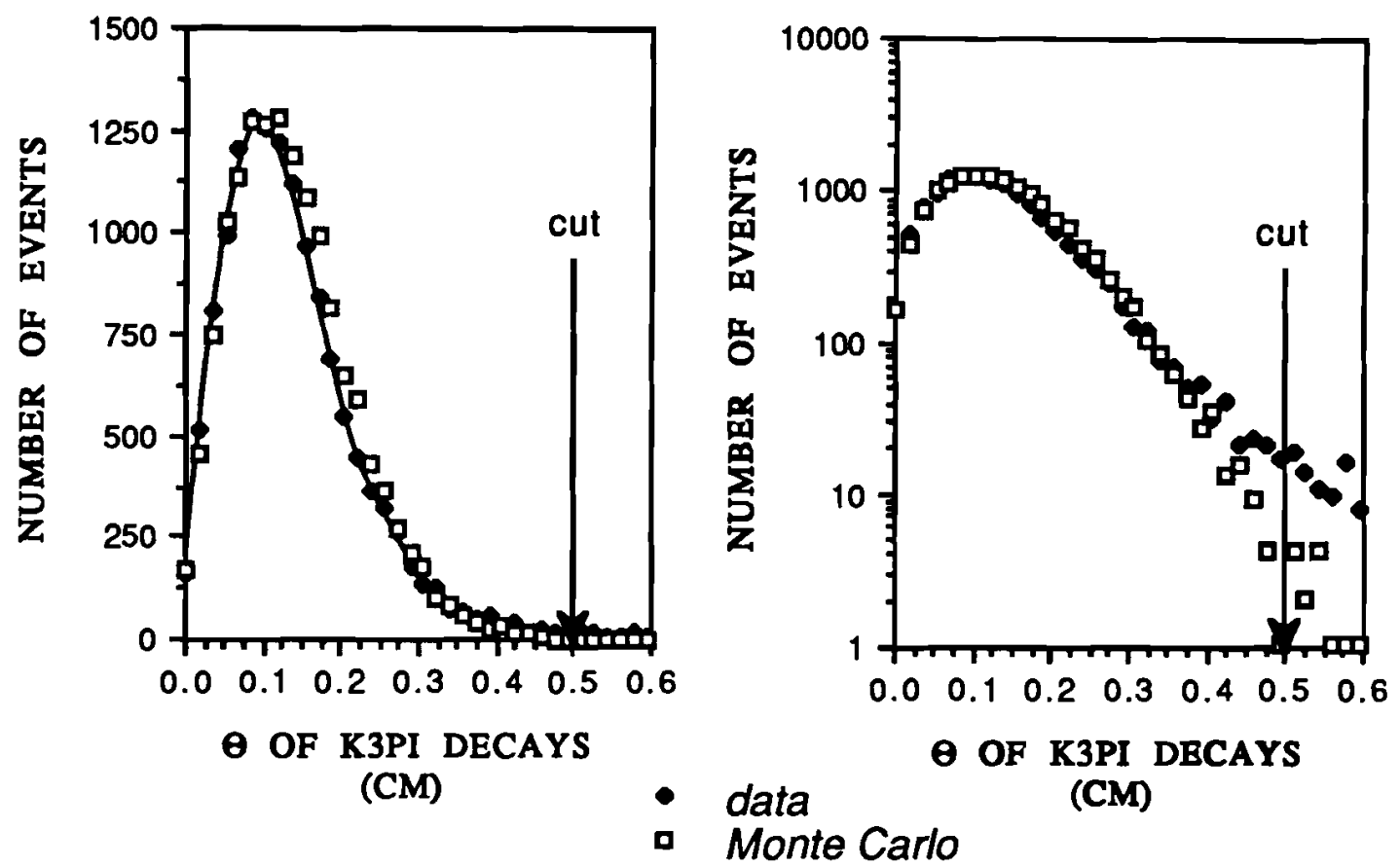

Figure 8.7 $\theta$ Radius for $\mathrm{K} \rightarrow 3 \pi$ Events from the Upstream West Target
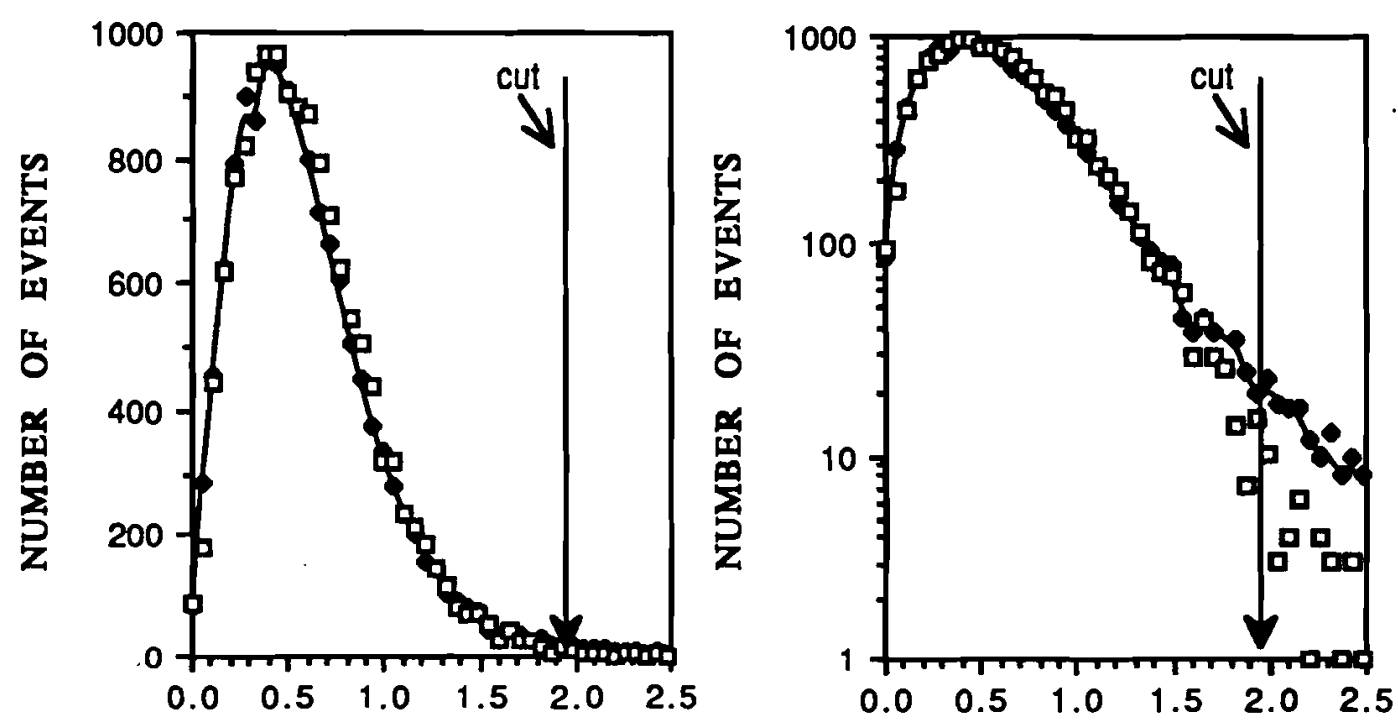

RADIUS AT UPSTREAM TARGET RADIUS AT UPSTREAM TARGET FOR K3PI DECAY - data (CM) - Monte Carlo FOR K3PI DECAY (CM)

Figure 8.8 Radius at the Upstream Target for $K \rightarrow 3 \pi$ Events from the Upstroam West Target 


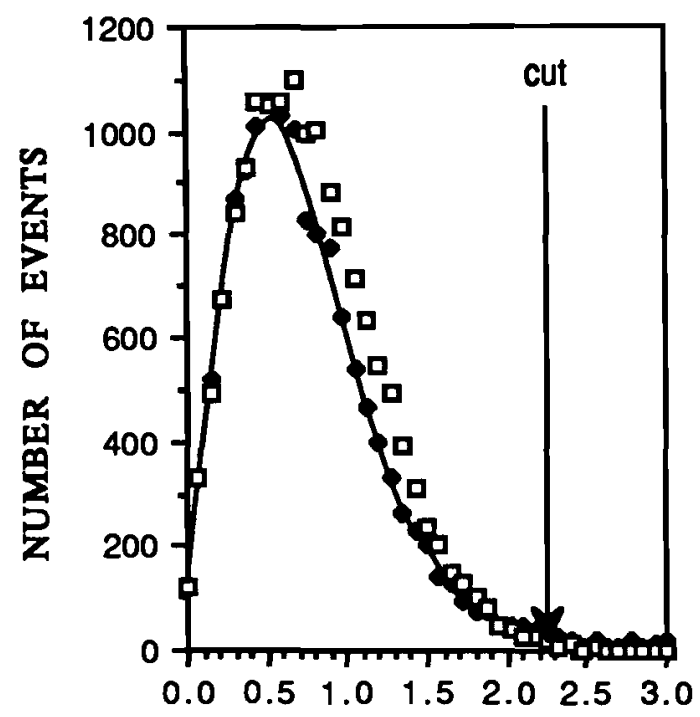

RADIUS AT LEAD GLASS

FOR KPI3 DECAY - data

(CM)

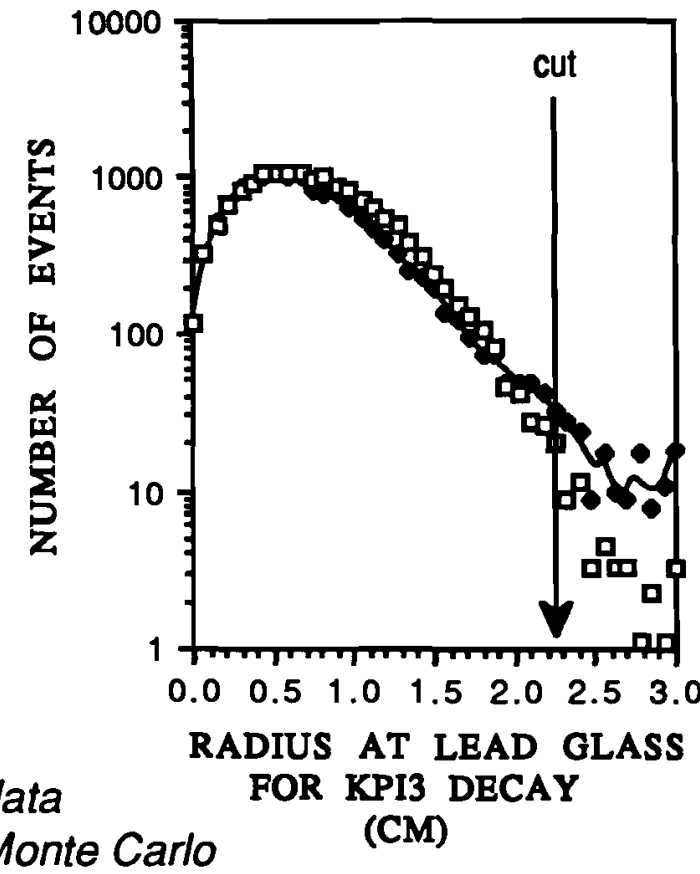

- Monte Carlo

Figure 8.9 Radius at the Glass for $\mathrm{K} \rightarrow 3 \pi$ Events from the Upstream West Target
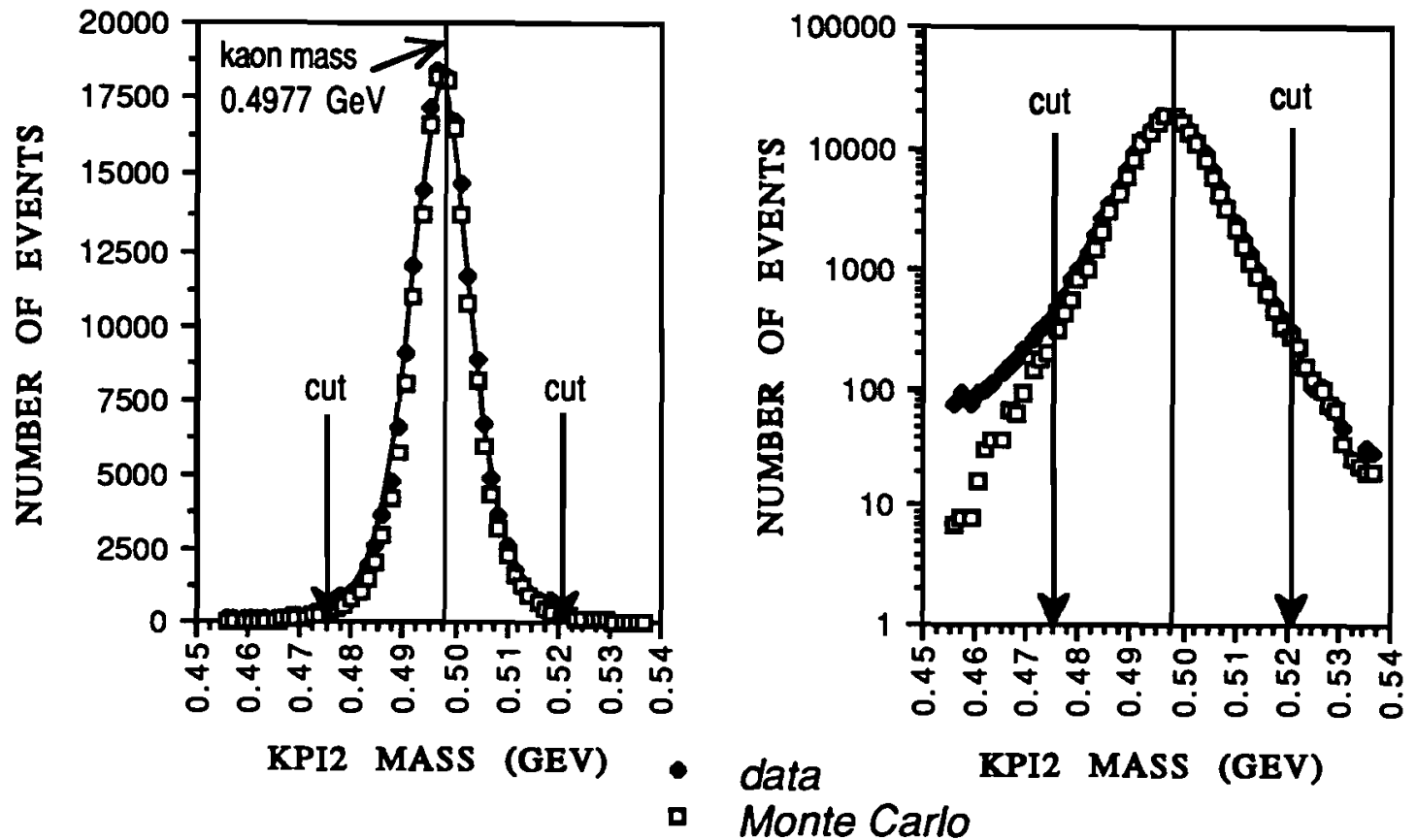

Figure $8.10 \mathrm{M}_{\pi^{+} \pi^{-}}$for $\mathrm{K} \rightarrow 2 \pi$ Events 


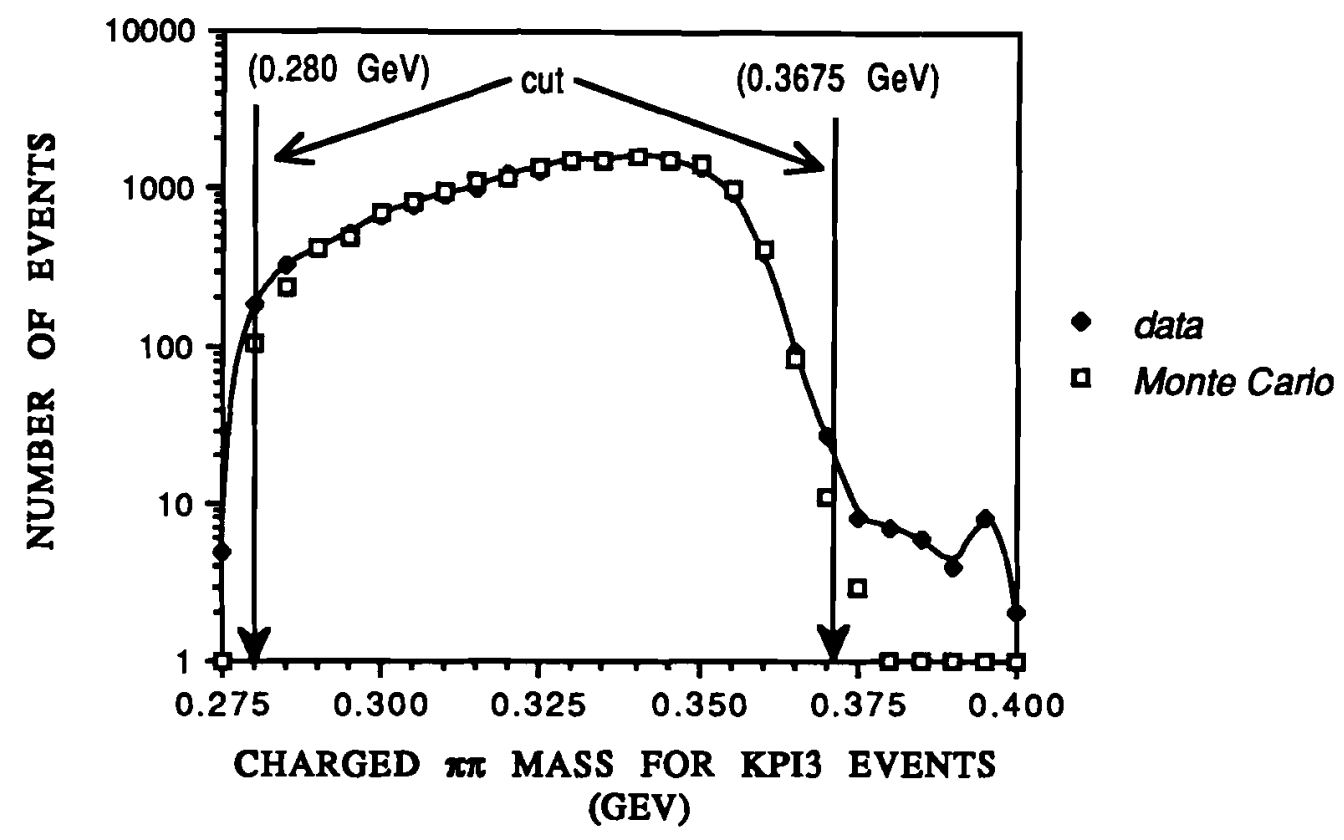

Figure $8.11 \mathrm{M}_{\pi^{+} \pi^{-}}$for $\mathrm{K} \rightarrow 3 \pi$ Events

The final $K_{2 \pi}$ data requirement was a kaon mass constraint. $\mathbf{M}_{\pi^{+} \pi^{-}}$for the $\mathrm{K} \rightarrow 2 \pi$ events was restricted to be between 0.475 and $0.521 \mathrm{GeV}$. The corresponding $\mathrm{K}_{3 \pi}$ mass constraint required $0.28<\mathrm{M}_{\pi^{+} \pi^{-}}<0.3675$. The $\mathrm{K}_{2 \pi}$ mass plot is shown using both a linear and a logarithmic scale in Figure 8.10. The charged $\pi^{+} \pi^{-}$mass for $\mathrm{K} \rightarrow 3 \pi$ decays is shown in Figure 8.11 .

After all the data selection criteia above were applied to the $K_{2 \pi}$ data sample, the $\mathrm{K}_{\mathrm{s}}$ lifetime was measured to be $0.0889 \pm 0.0002$, within one standard deviation of the world average ${ }^{(41)}$ value of $0.0892 \pm 0.0002$ nsec. Comparison plots of the final $K_{2 \pi}$ sample are given below for the $z$ of the decay vertex, the proper time distribution, the momentum of the $\pi^{+}$, the momentum of the $\pi^{-}$and the charged track opening angle. The curves are drawn through the data points. The Monte Carlo program assumes the world average value for the $K_{s}$ lifetime when generating events. 


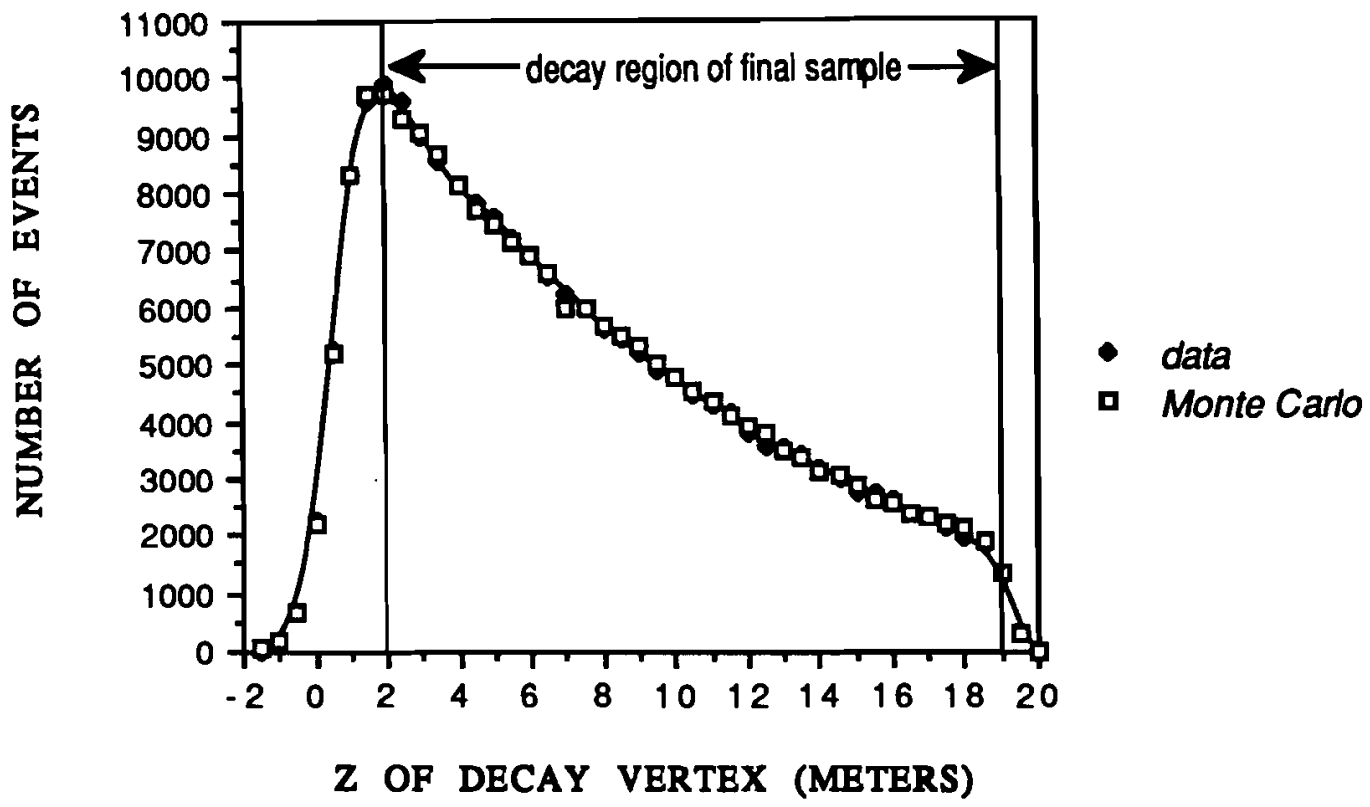

Figure 8.12 $Z$ Vertex of $K_{2 \pi}$ Decay

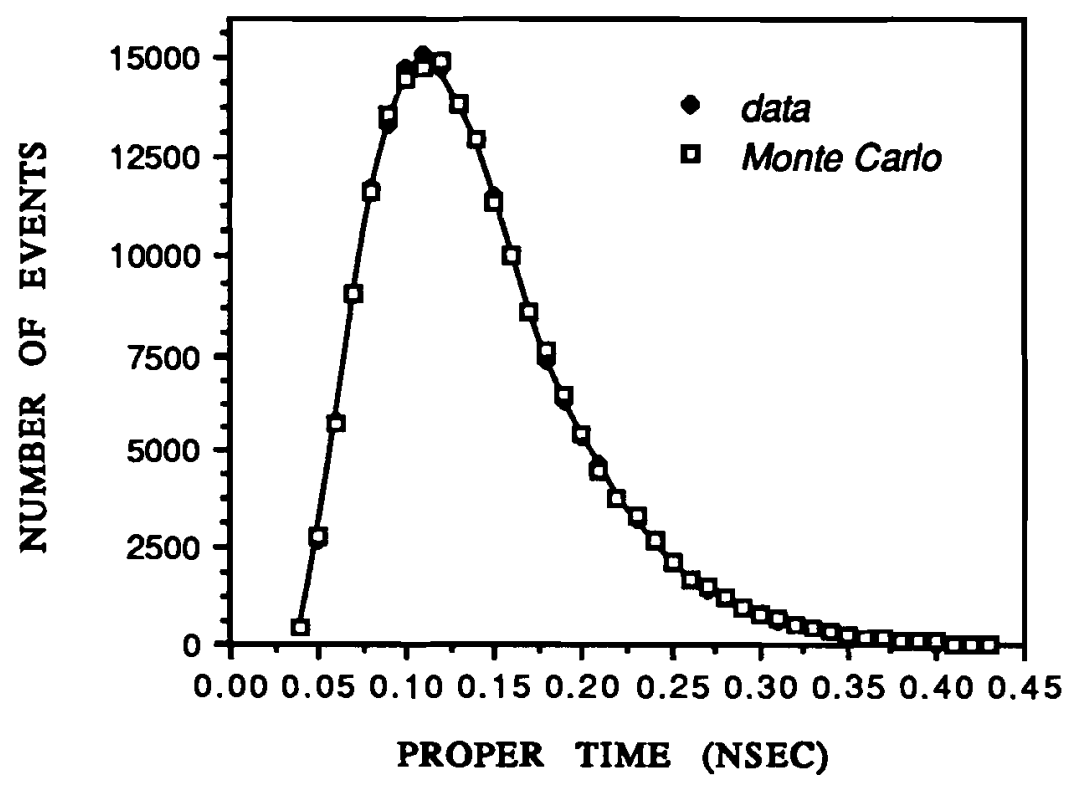

Flgure 8.13 Proper TIme of $\mathrm{K}_{2 \pi}$ Decay 


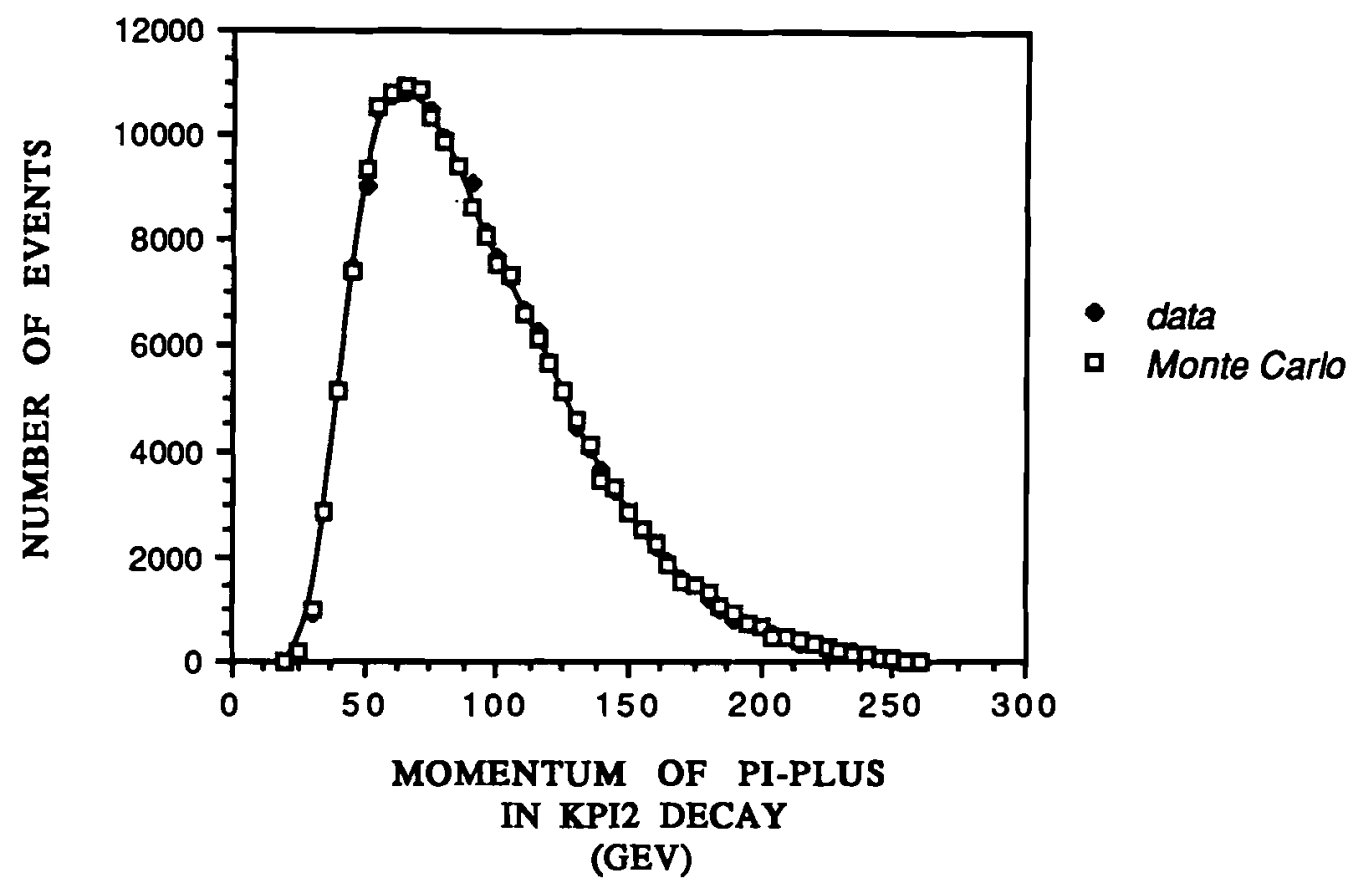

Figure 8.14 Momentum of $\pi^{+}$from $\mathrm{K}_{2 \pi}$ Decay

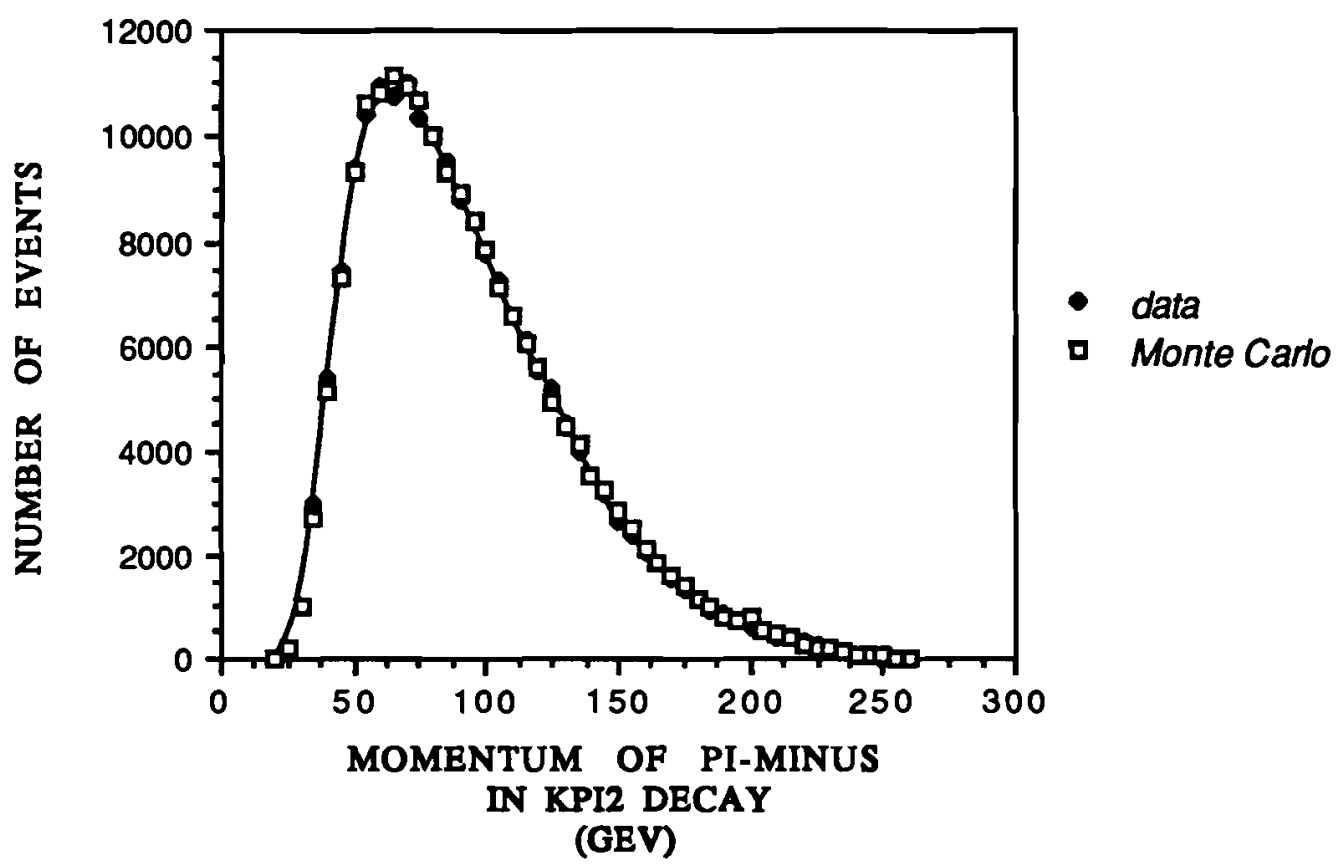

Figure 8.15 Momentum of $\pi^{-}$from $\mathrm{K}_{2 \pi}$ Decay 


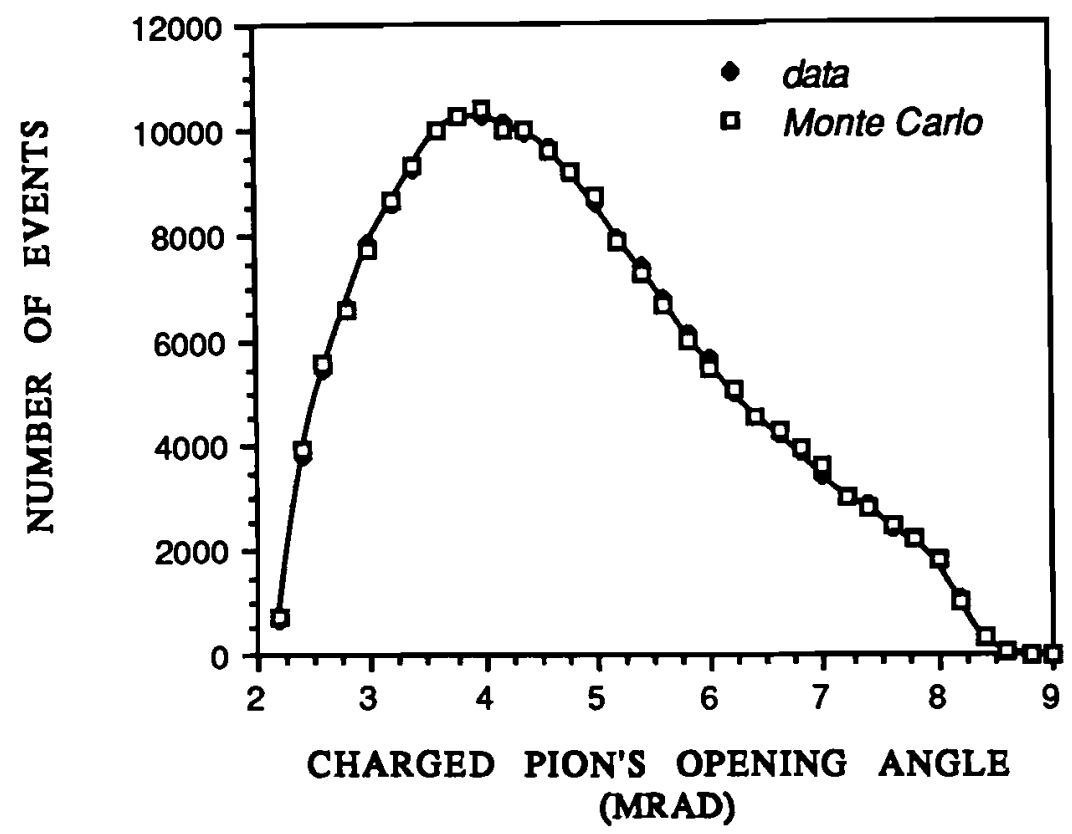

Flgure 8.16 Charged Track Opening Angle In $\mathrm{K}_{2 \pi}$ Decay

\subsection{Further $\mathrm{K}_{3 \pi}$ Data Requirements}

The $\mathrm{K}_{2 \pi}$ and $\mathrm{K}_{3 \pi}$ momentum spectra at the target must be the same since both are samples of the kaon production momentum spectrum. The kaon spectrum for the $\mathrm{K}_{3 \pi}$ data was determined by correcting the $\mathrm{K}_{2 \pi}$ data sample using the acceptance determined by the Monte Carlo and subtracting $\mathrm{K}_{2 \pi}$ collimator production background (see Appendix C).

When this kaon spectrum was used in the $\mathrm{K}_{3 \pi}$ Monte Carlo, it agreed with the $\mathrm{K}_{3 \pi}$ data above $130 \mathrm{GeV}$. Below $130 \mathrm{GeV}$ there was an excess of Monte Carlo events. The detected momentum spectrum for the final $\mathrm{K}_{2 \pi}$ and $\mathrm{K}_{3 \pi}$ event sample is shown in Figures 8.17 and 8.18 .

The requirement that the reconstructed kaon momentum be between 130-350 $\mathrm{GeV}$ assured agreement between $\mathrm{K}_{3 \pi}$ Monte Carlo and data momentum spectrums while still using the same spectrum in the $\mathrm{K}_{2 \pi}$ and $\mathrm{K}_{3 \pi}$ Monte Carlos. 


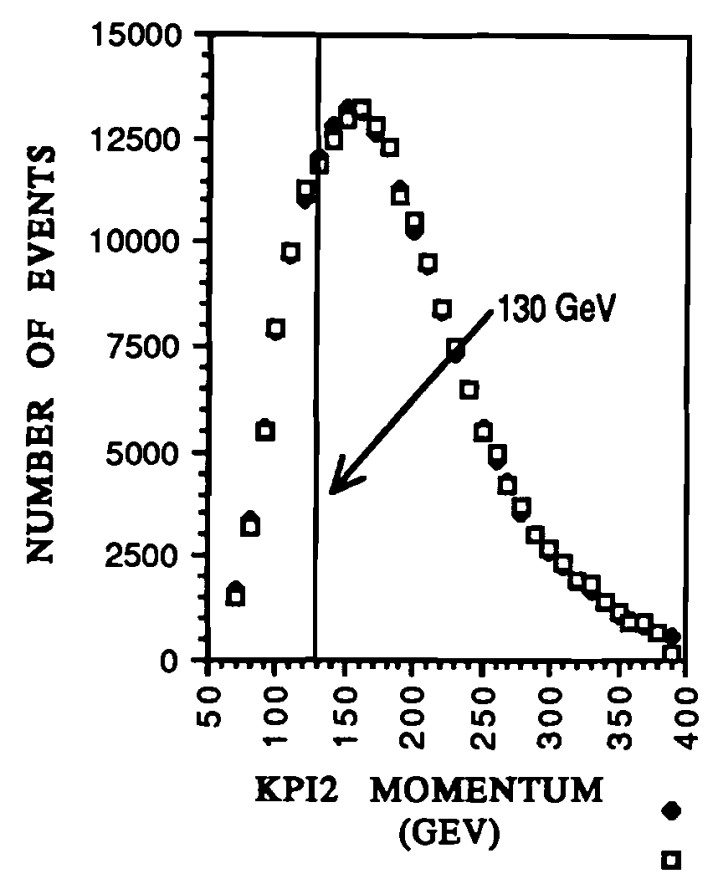

Figure $8.17 \mathrm{~K}_{2 \pi}$ Momentum Spectrum

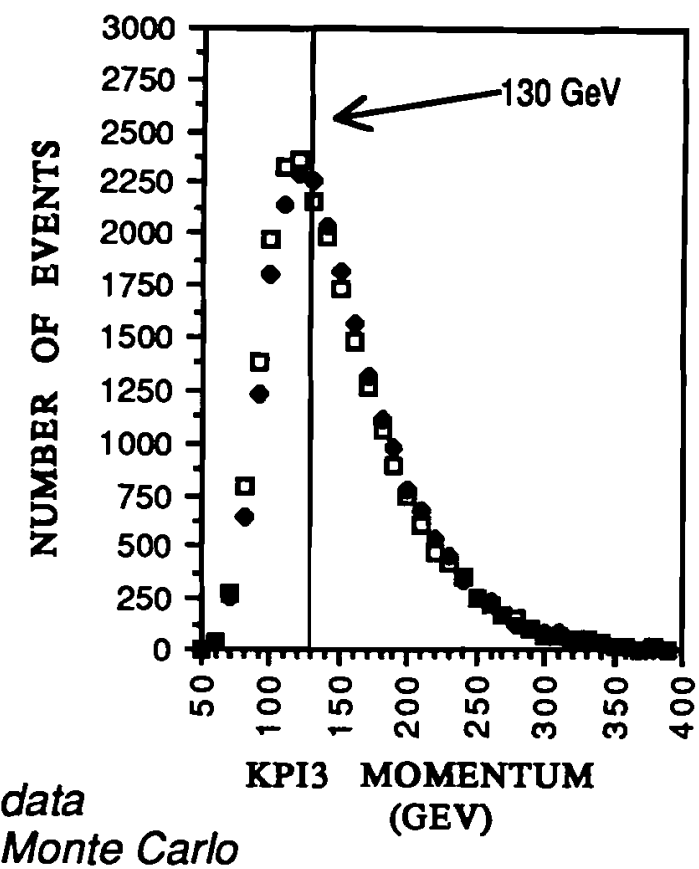

Figure $8.18 \mathrm{~K}_{3 \pi}$ Momentum Spectrum

\subsection{1 $\mathrm{K}_{3 \pi}$ Photon Selection Criteria}

The $\pi^{0}$ in $K_{3 \pi}$ decay sublty effects the momentum and vertex of the parent $K_{3 \pi}$ through its acceptance. The acceptance of the $\pi^{\mathbf{0}}$ and the CMR Trigger Processor were the only differences between $K_{2 \pi}$ and $K_{3 \pi}$ triggers. Several "cleanliness" requirements were applied to the photon showers with poor resolution in position and/or energy.

Low energy photon showers had potential to cause poorly reconstructed events. Requiring that each photon had energy greater than $3.5 \mathrm{GeV}$ eliminated photon showers which were hard to simulate in the Monte Carlo while losing less than $1 \%$ of the data. Figures 8.19 and 8.20 show this $3.5 \mathrm{GeV}$ cut for both showers on linear and logarithmic scales.

Events with either photon within $2.54 \mathrm{~cm}$ of the hole in the lead glass array were also removed as were events with showers that shared a nearest neighbor block with another neutral or charged shower. These events had poor resolution and were difficult to simulate in the Monte Carlo. Some data events had more than two neutral showers. These extra neutral showers could cause an incorrect selection of which 


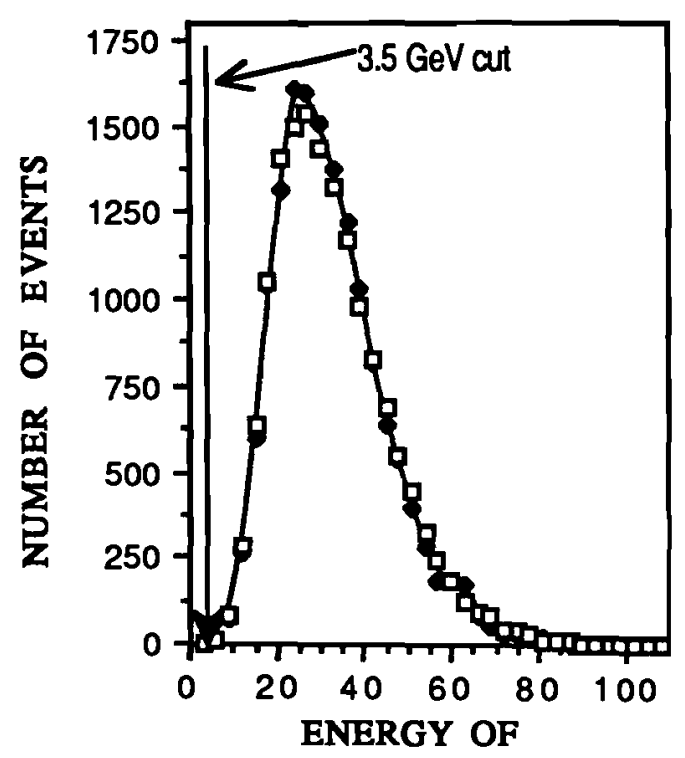

HIGHER ENERGY PHOTON

(GEV)

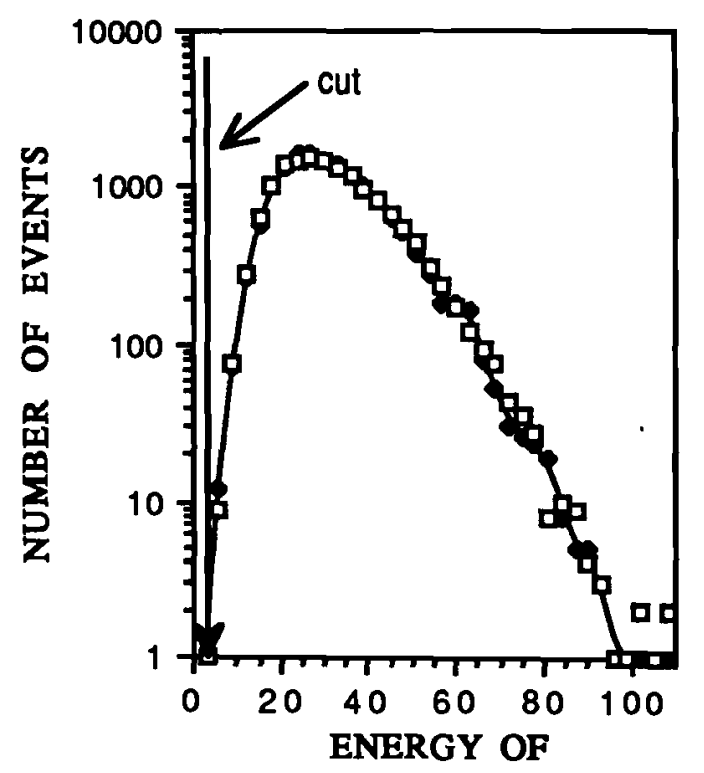

HIGHER ENERGY PHOTON data

Monte Carlo

(GEV)

Figure 8.19 Photon Energy Criterion: Higher Energy Photon

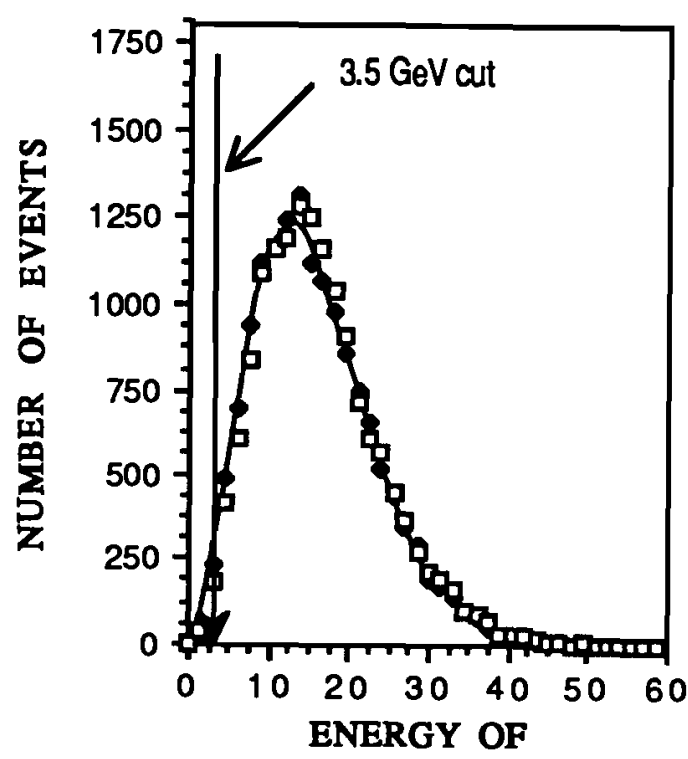

LOWER ENERGY PHOTON (GEV)

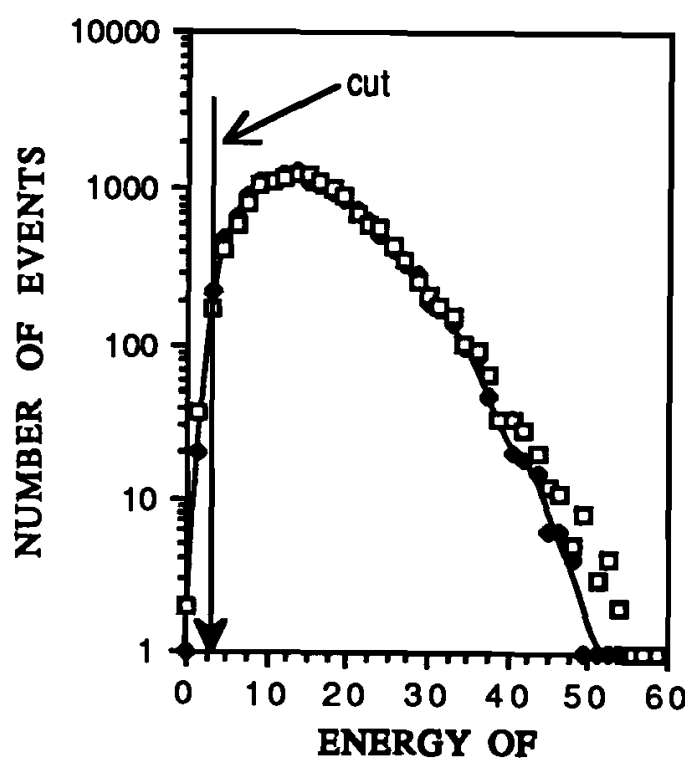

LOWER ENERGY PHOTON (GEV)

Figure 8.20 Photon Energy Criterion: Lower Energy Photon 
two showers corresponded to the $\pi^{\circ}$ photons. The energy distribution of these extra neutral showers was shown in Figure 6.24. For events where the extra shower energy is small, the chance of mis-identifying the correct showers is negligible. For this reason, events with extra neutral showers whose energy was greater than $5 \mathrm{GeV}$ were removed from the $K_{3 \pi}$ data sample. These shower requirements and the percentage of events they removed are summarized in Table 8.3.

\begin{tabular}{|c|c||c|}
\hline Cut & Data Lost & $\begin{array}{c}\text { Monte Carlo } \\
\text { Lost }\end{array}$ \\
\hline energy > 3.5GeV & $<0.5 \%$ & $<0.5 \%$ \\
\hline $\begin{array}{c}\text { neither within } 2.54 \mathrm{~cm} \\
\text { of the hole }\end{array}$ & $13 \%$ & $10 \%$ \\
\hline $\begin{array}{c}\text { energy of extra neutral } \\
\text { showers < 5GeV }\end{array}$ & $3.2 \%$ & $0 \%$ \\
\hline $\begin{array}{c}\text { showers not overlap a } \\
\text { neutral shower }\end{array}$ & $4.4 \%$ & $2.3 \%$ \\
\hline $\begin{array}{c}\text { showers not overlap a } \\
\text { charged shower }\end{array}$ & $1.3 \%$ & $0 \%$ \\
\hline
\end{tabular}

Table 8.3 Photon Shower Selection Criterion

After applying these photon cleanliness criteria, Monte Carlo and data photon distributions matched well for both hole and non-hole evnets. Monte Carlo and data comparison plots are shown in Figures 8.21-8.25 for the $\pi^{0}$ mass, photon energies of each shower, number of blocks in each shower, photon-photon opening angle and $\pi^{0}$ momentum. Comparisons are shown for events where both photons showered more than $5.58 \mathrm{~cm}$ from the hole in the array (non-hole events) and for events where both photons showered within $5.58 \mathrm{~cm}$ of the hole in the array (hole events). The curves are drawn through the data points. 


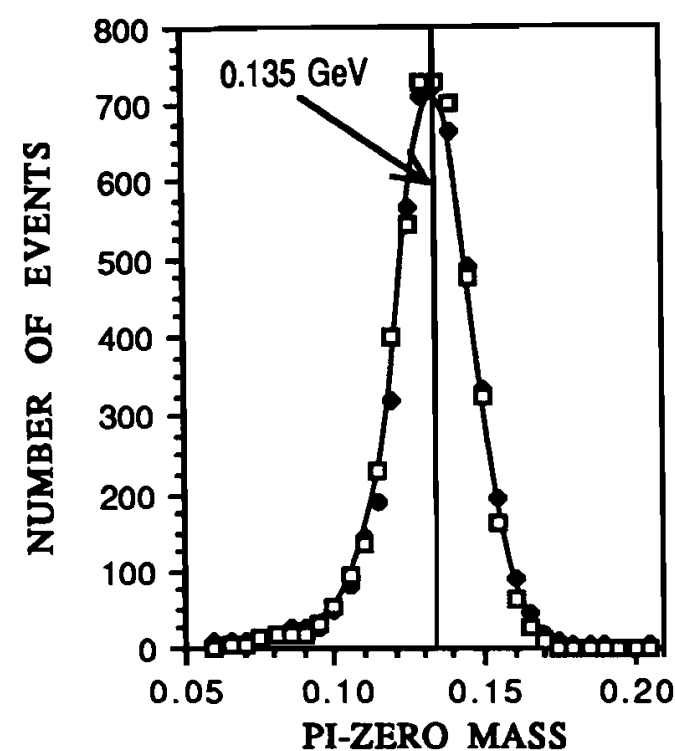

FOR NON-HOLE EVENTS (GEV)

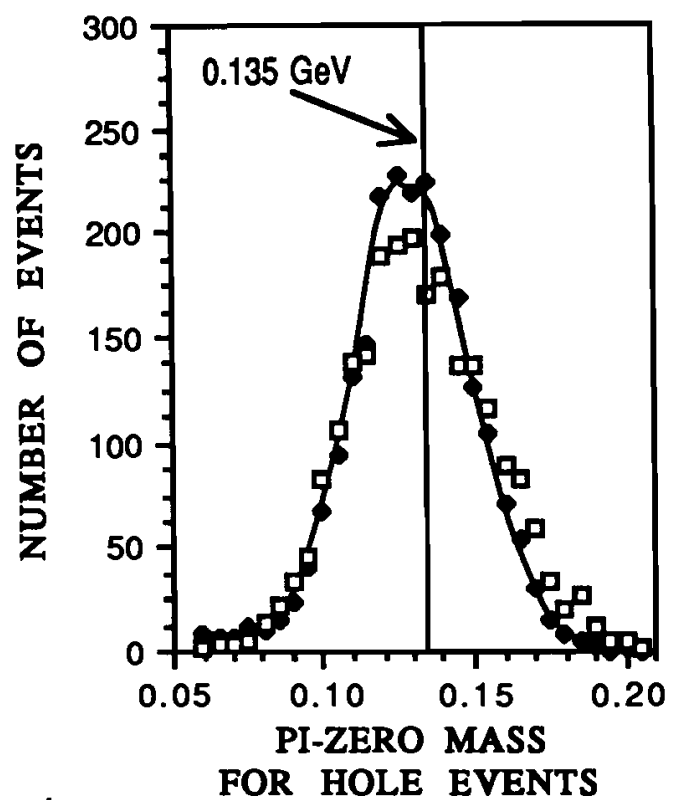

- data (GEV)

\section{- Monte Carlo}

Flgure 8.21 Mass of the $\pi^{0}$ (non-hole and hole events)

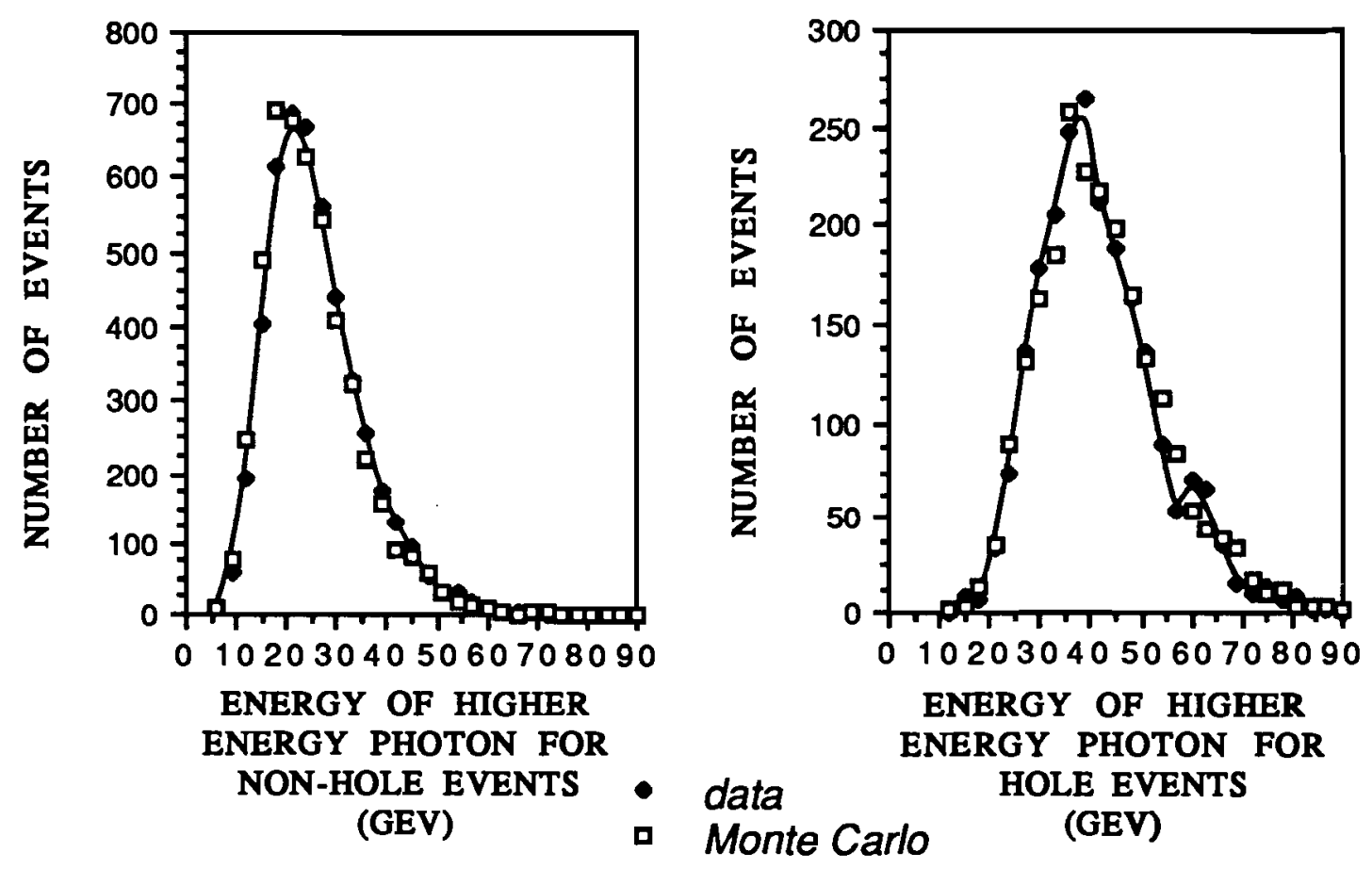

Figure 8.22a Energy of the Higher Energy Shower (non-hole and hole events) 

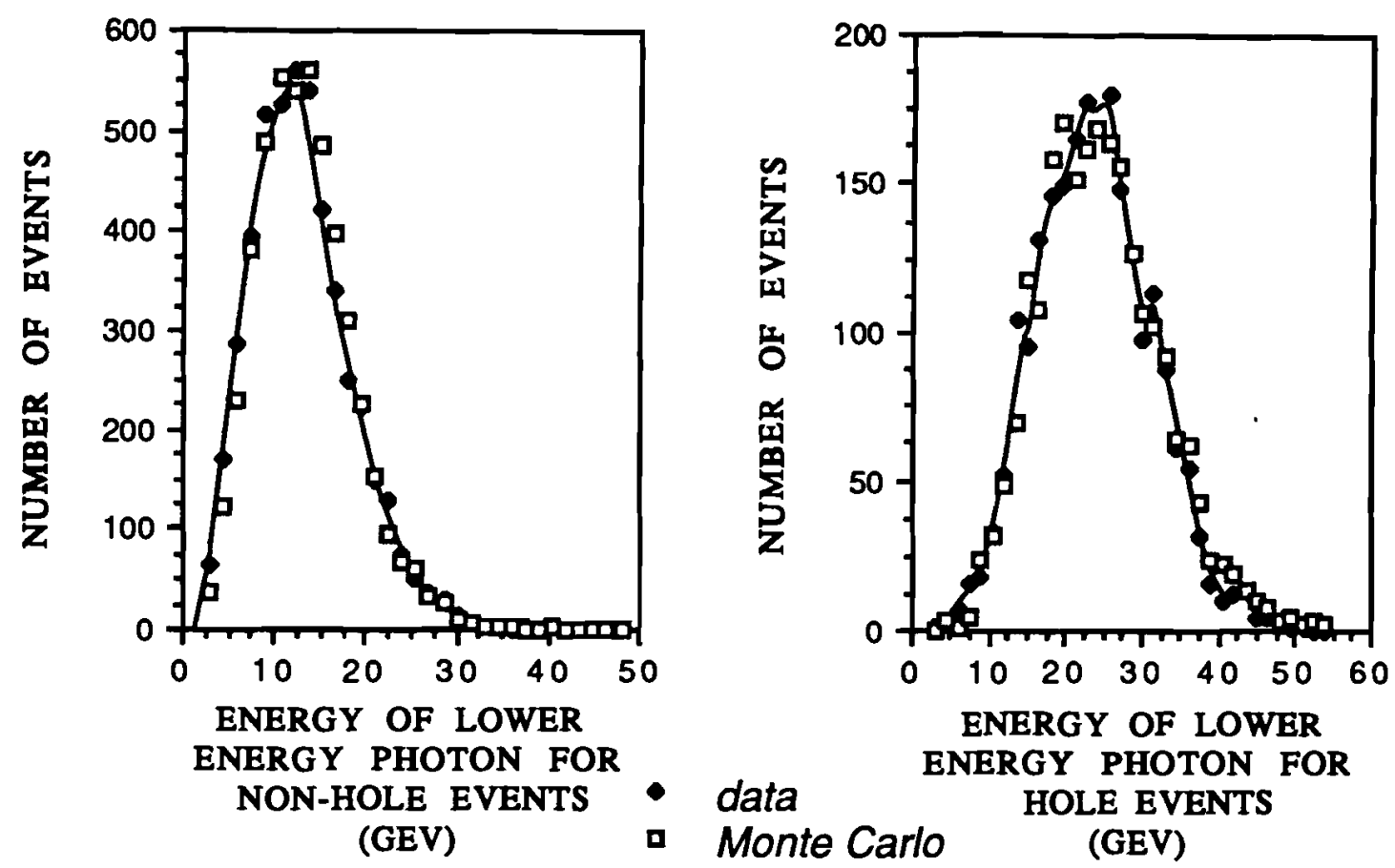

Figure 8.22b Energy of the Lower Energy Shower (non-hole \& hole events)

In Higher Energy Shower:

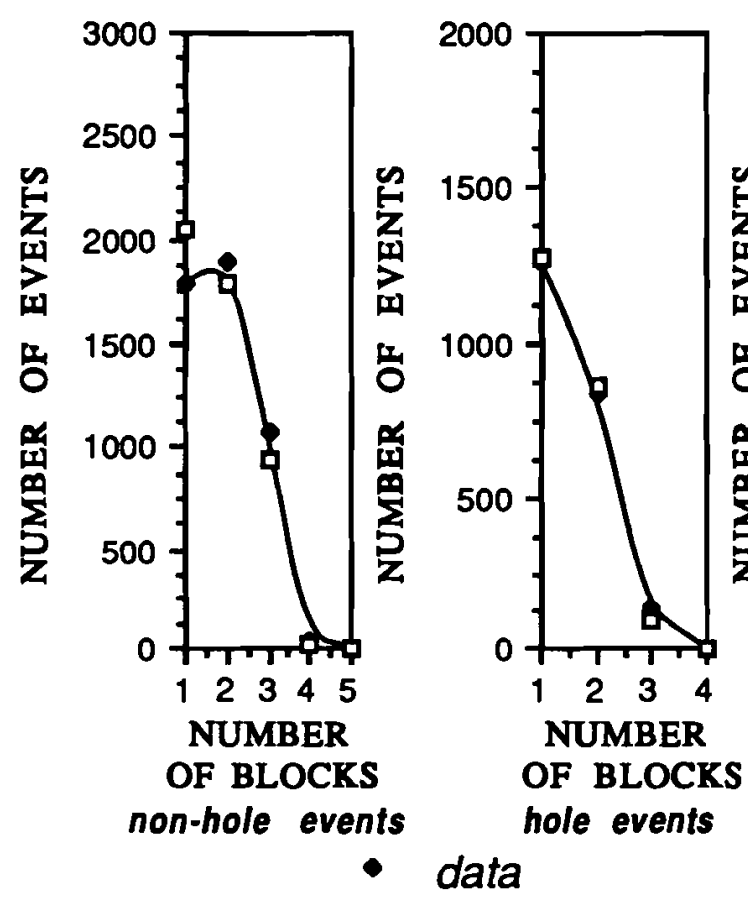

In Lower Energy Shower:

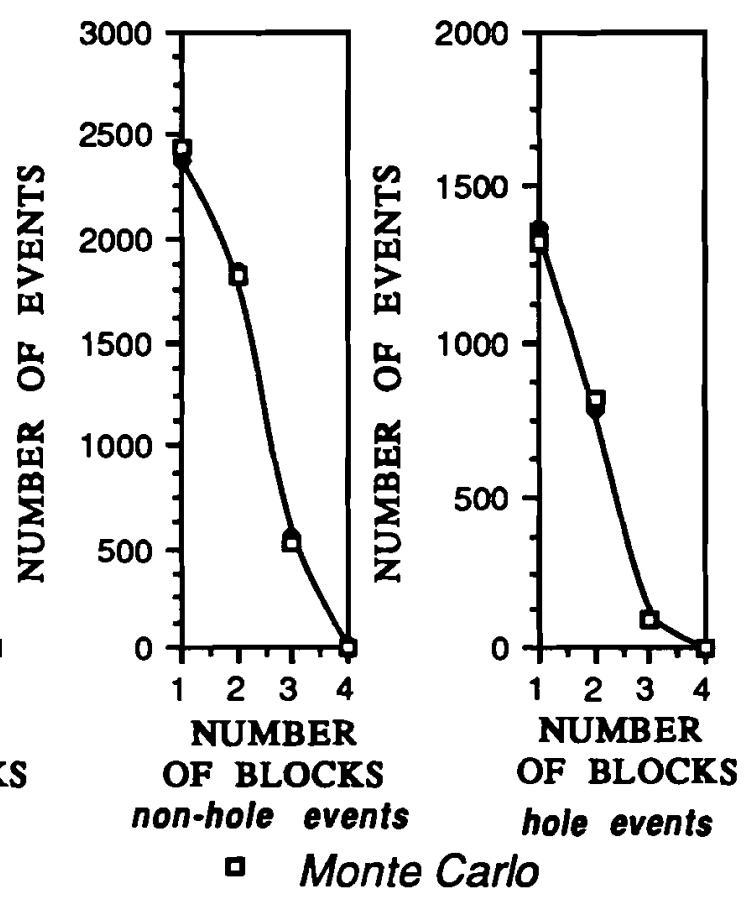

Figure 8.23 Number of Blocks in Showers (non-hole and hole events) 


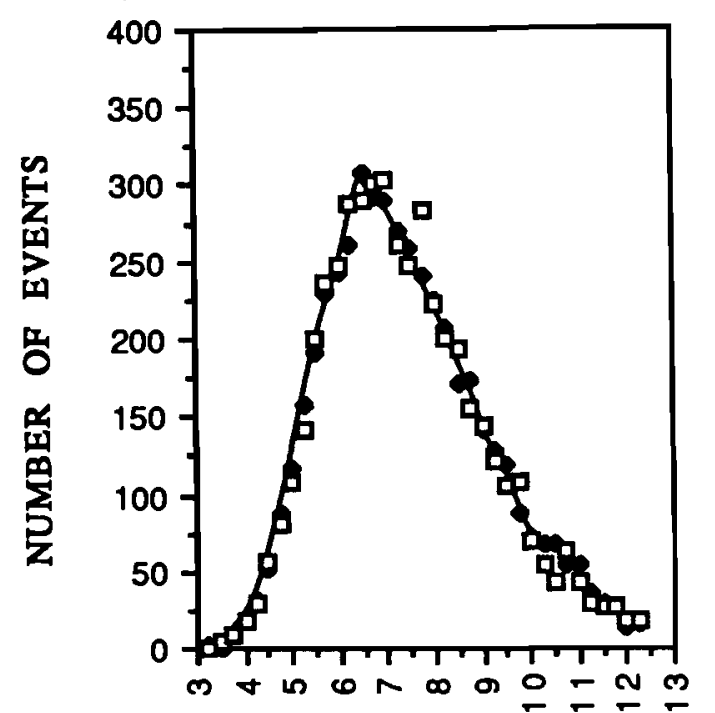

PHOTON-PHOTON OPENING ANGLE: NON-HOLE EVENTS (MR)

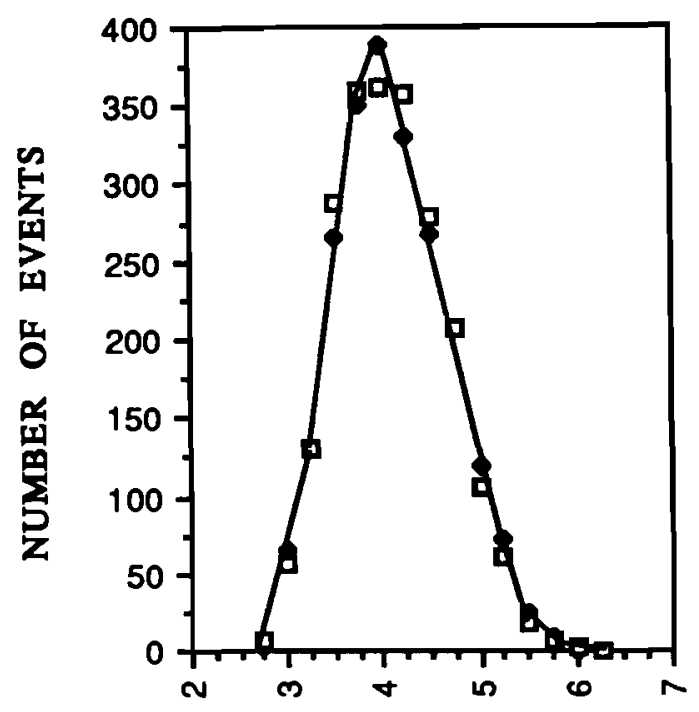

PHOTON-PHOTON OPENING ANGLE: HOLE EVENTS

- data ANGLE: HR)

Figure 8.24 Photon-Photon Opening Angle (non-hole and hole events)

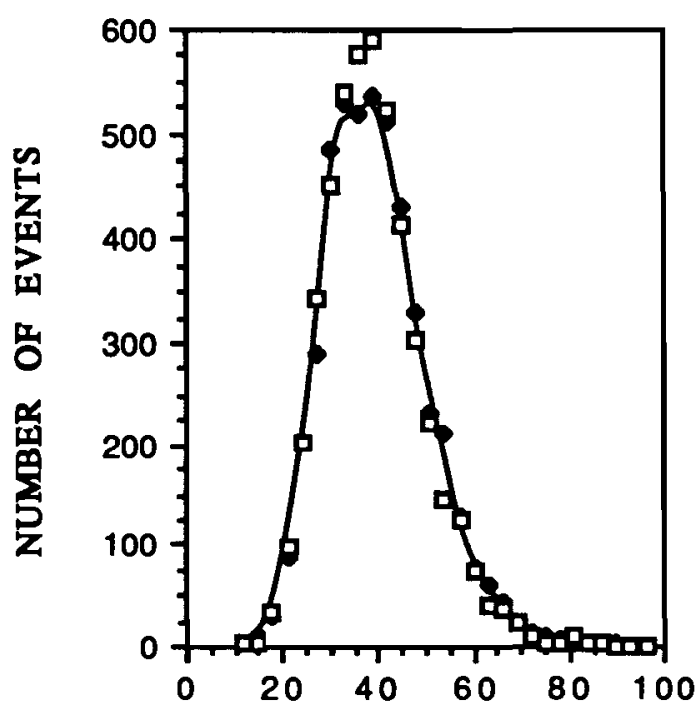

PI-ZERO MOMENTUM: NON-HOLE EVENTS (GEV)

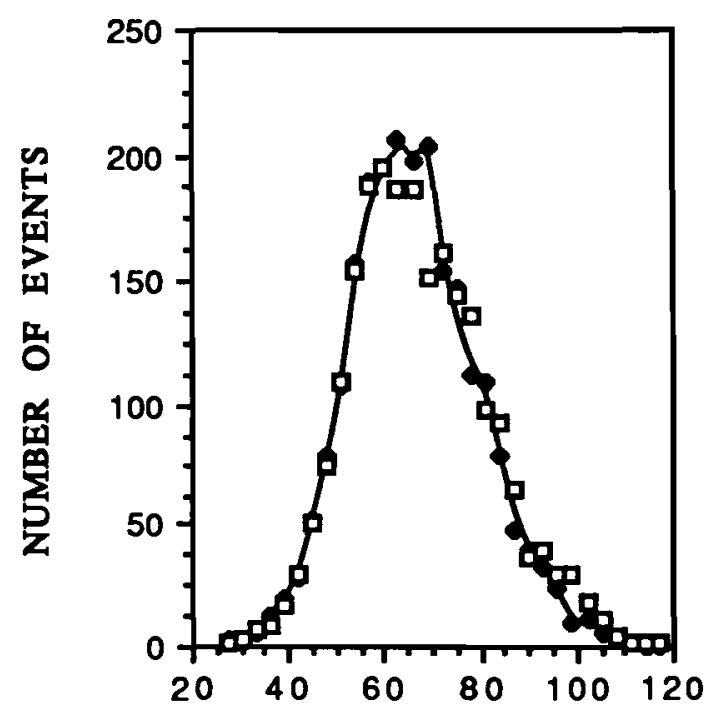

PI-ZERO MOMENTUM:

- data HOLE EVENTS

- Monte Carlo (GEV)

Figure 8.25 Momentum of the $\pi^{\circ}$ (non-hole and hole events) 


\subsection{2 $\mathrm{K}_{3 \pi}$ Final Data Selection}

The $z$ of the $\mathrm{K}_{3 \pi}$ decay vertex did not match between Monte Carlo, as evidenced by Figure 8.29 , even when all final $K_{3 \pi}$ selection criteria had been applied. Yet for $K_{2 \pi}$ decay the $z$ vertex distribution agreed beautifully betwen Monte Carlo and data (see Figure 8.12). For this reason I investigated $\Xi^{0} \rightarrow \Lambda \pi^{0} \rightarrow p \pi^{-} \pi^{0}$ and $\mathrm{K}_{\mathrm{s}}{ }^{0} \rightarrow \pi^{0} \pi^{0}$ $\rightarrow \mathrm{e}^{+} \mathrm{e}^{-} \gamma \pi^{0}$ (where the $\pi^{0}$ decays immediately into two photons) backgrounds. I also compared Monte Carlo and data Dalitz distributions in hopes of improving the $z$ vertex discrepancys. Unfortunately none of these investigations were fruitful.

The $\mathrm{K}_{\mathrm{s}}{ }^{0} \rightarrow \mathrm{e}^{+} \mathrm{e}^{-3} \gamma$ background is visible if one reconstructs the two particle charged mass as an $\mathrm{e}^{+} \mathrm{e}^{-}$pair as shown in Figure $8.26 \mathrm{a}$ and $\mathrm{b}$ on a linear and logarithmic scale respectively. All other selection criteria have been applied to the data and Monte Carlo events in the sample shown in Figure 8.26.

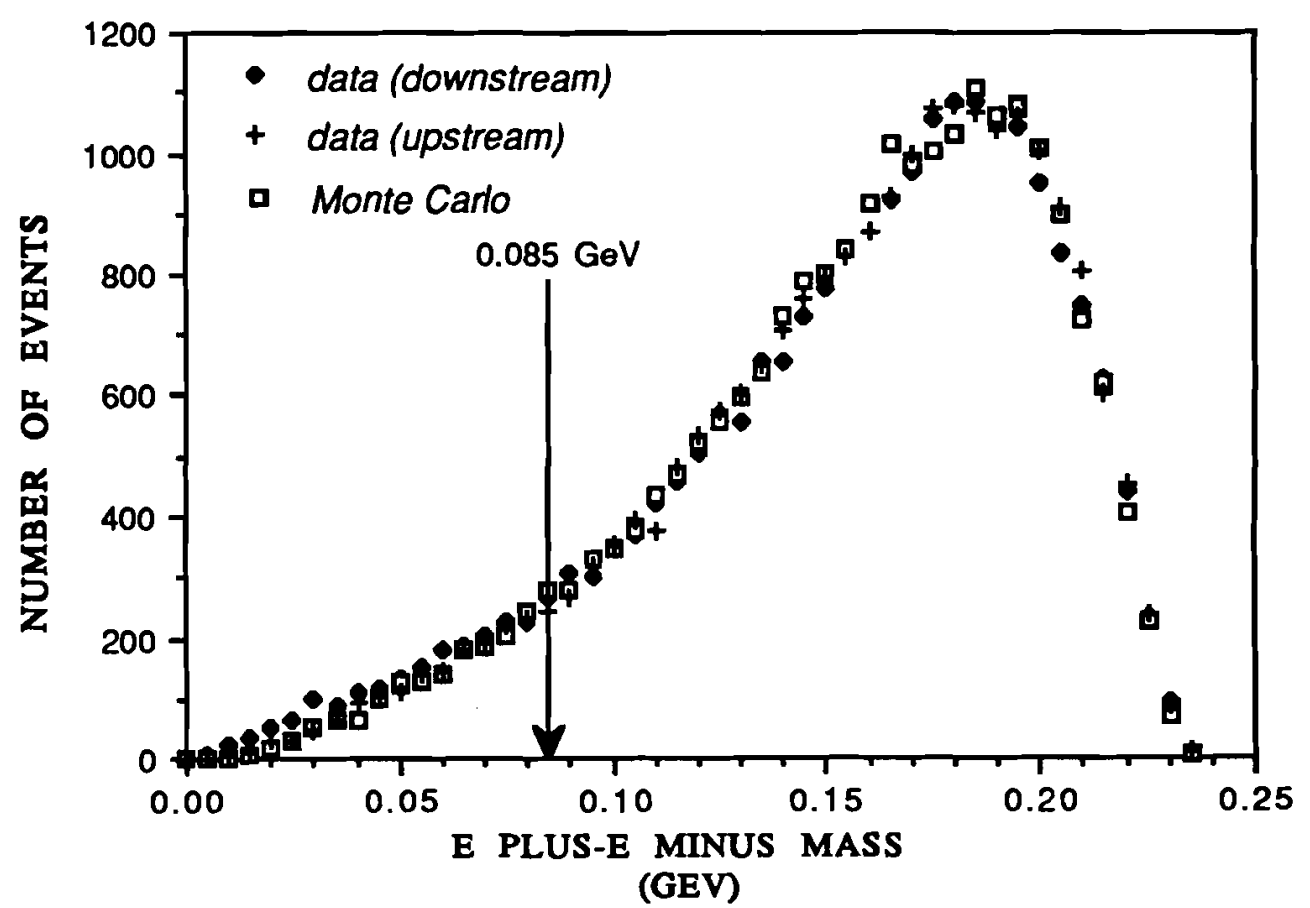

Figure 8.26a Mass of Two Charged Tracks in $\mathrm{K}_{3 \pi}$ Data Sample Reconstructed as $\mathrm{e}^{+} \mathrm{e}^{-}$(linear scale, uncut)

Figures 8.26a and $b$ show the $\mathrm{e}^{+} \mathrm{e}^{-}$mass for the upstream and downstream data and a downstream Monte Carlo on linear and logarithmic scales. These $K_{s}$ events are 
potentially dangerous as they would look like a $\mathrm{K}_{\mathrm{s}}^{0} \rightarrow \pi^{+} \pi^{-} \pi^{0}$ signal. Due to the electrons small mass none of these events have $\mathrm{M}_{\mathrm{e}} \mathrm{e}^{-}$above $0.14 \mathrm{GeV}$ so that a cut at $0.14 \mathrm{GeV}$ removes all ot this background while reducing both the $\mathrm{K}_{3 \pi}$ data and Monte Carlo samples by $33 \%$. While the requirement that the $\mathrm{e}^{+} \mathrm{e}^{-}$mass be above 0.14 $\mathrm{GeV}$ removes all the $\mathrm{K}_{\mathrm{s}}{ }^{0} \rightarrow \mathrm{e}^{+} \mathrm{e}^{-} 3 \gamma$ decays from the final data sample, it is a rather severe requirement. A looser requirement of $\mathrm{e}^{+} \mathrm{e}^{-}$mass greater than $0.085 \mathrm{GeV}$ did not effect any of the final measurements of $\eta_{+-0}$ by more than $1 / 4$ a standard deviation (see Section 9.5.6) or improve the $z$ vertex discrepancy, and thus this looser requirement was used in the final analysis. With $\mathrm{e}^{+} \mathrm{e}^{-}$mass restricted to be greater than $0.085 \mathrm{GeV}$ it was calculated, using a Monte Carlo simulation, that less than $0.2 \% \mathrm{~K}_{\mathrm{s}}{ }^{0} \rightarrow \mathrm{e}^{+} \mathrm{e}^{-3} \gamma$ events remained in the final data sample.

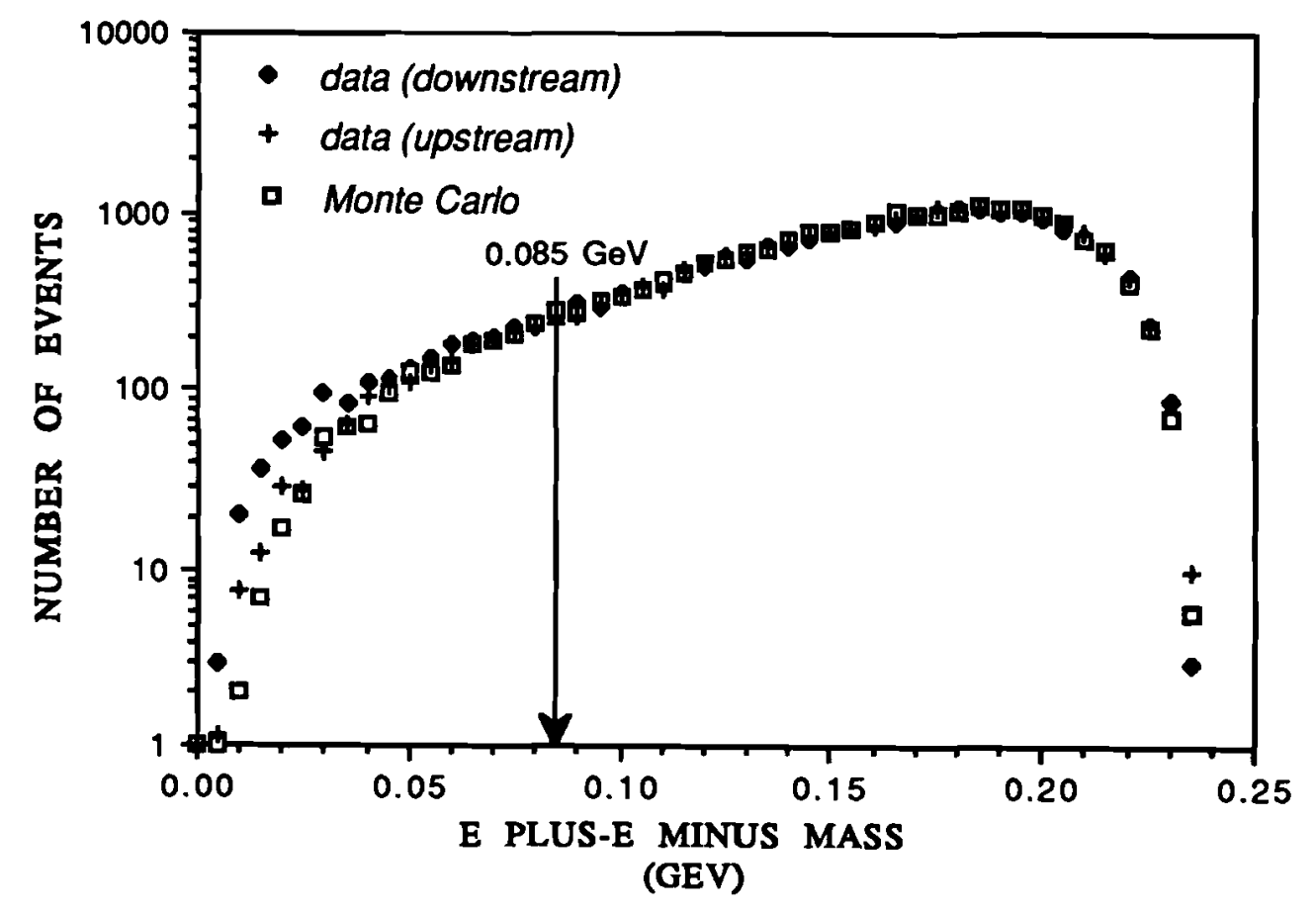

Figure 8.26b Mass of Two Charged Tracks in $K_{3 \pi}$ Data Sample Reconstructed as $\mathrm{e}^{+} \mathrm{e}^{-}$(logarithmic scale, uncut) 
Next I investigated possible $\Xi^{0}$ backgrounds. A preliminary $\Lambda^{0}$ mass cut at $3 \sigma$ was increased to approximately $10 \sigma$, requiring $M_{p \pi}$ be outside the limits 1.0856 GeV and 1.1456 GeV. A lambda mass plot was shown in Figure 6.17. Similarly the allowed charged momentum ratio $P_{\pi^{+}} / P_{\pi^{-}}$was tightened to $0.4<\mathrm{P}_{\pi^{+}} / \mathrm{P}_{\pi^{-}<2.5}$ in order to reduce the acceptance of $\Lambda^{0} \mathrm{~s}$. The lambda mass and charged momentum ratio requirements were redundant and removed only $1.4 \%$ of the $\mathrm{K}_{3 \pi}$ data and Monte Carlo events. As with the tighter $\mathrm{e}^{+} \mathrm{e}^{-}$mass requirement, the tightening of these two selection criteria had little effect on the final measurement of $\eta_{+-0}$ (see Section 9.5.6) or on the decay vertex distribution. Thus these requirements were relaxed back to their previous limits of $3 \sigma$ for the lambda mass and 0.3 to 3.0 for $\mathrm{P}_{\pi^{+}} / \mathrm{P}_{\pi^{-}}$.

$\mathrm{K}_{3 \pi}\left(0.43 \mathrm{GeV}<\mathrm{M}_{\mathrm{K} 3 \pi}<0.565 \mathrm{GeV}\right)$ and $\pi^{0}\left(0.60 \mathrm{GeV}<\mathrm{M}_{\pi^{0}}<0.210 \mathrm{GeV}\right)$ mass constraints were very loose as data and Monte Carlo comparisons did not indicate any background present in the final data sample. The $\mathrm{K}_{3 \pi}$ and $\pi^{0}$ mass plots are shown in Figures 8.27 and 8.28 on both linear and logarithmic scales.

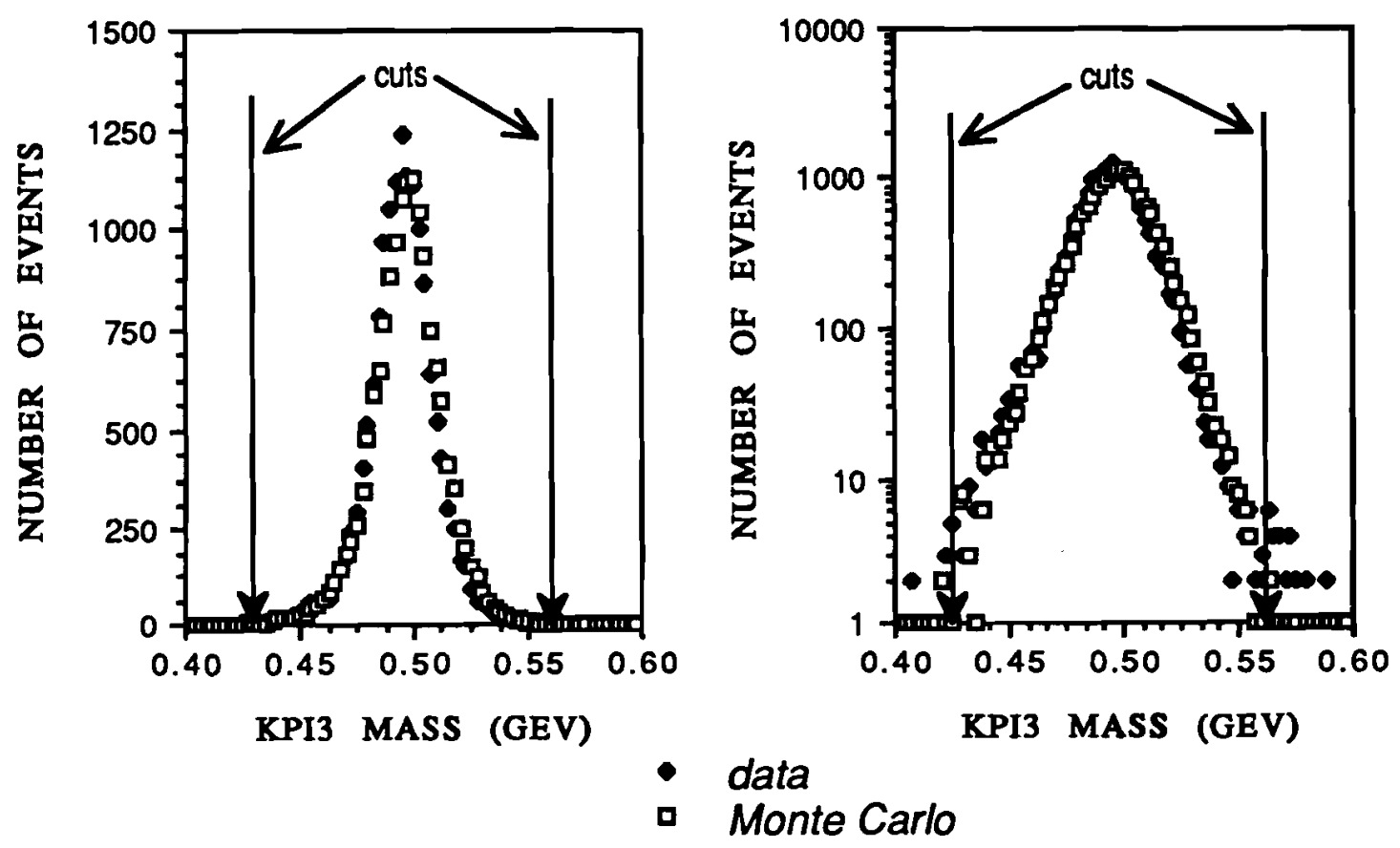

Figure 8.27 Mass of $\mathrm{K}_{3} \pi$ 


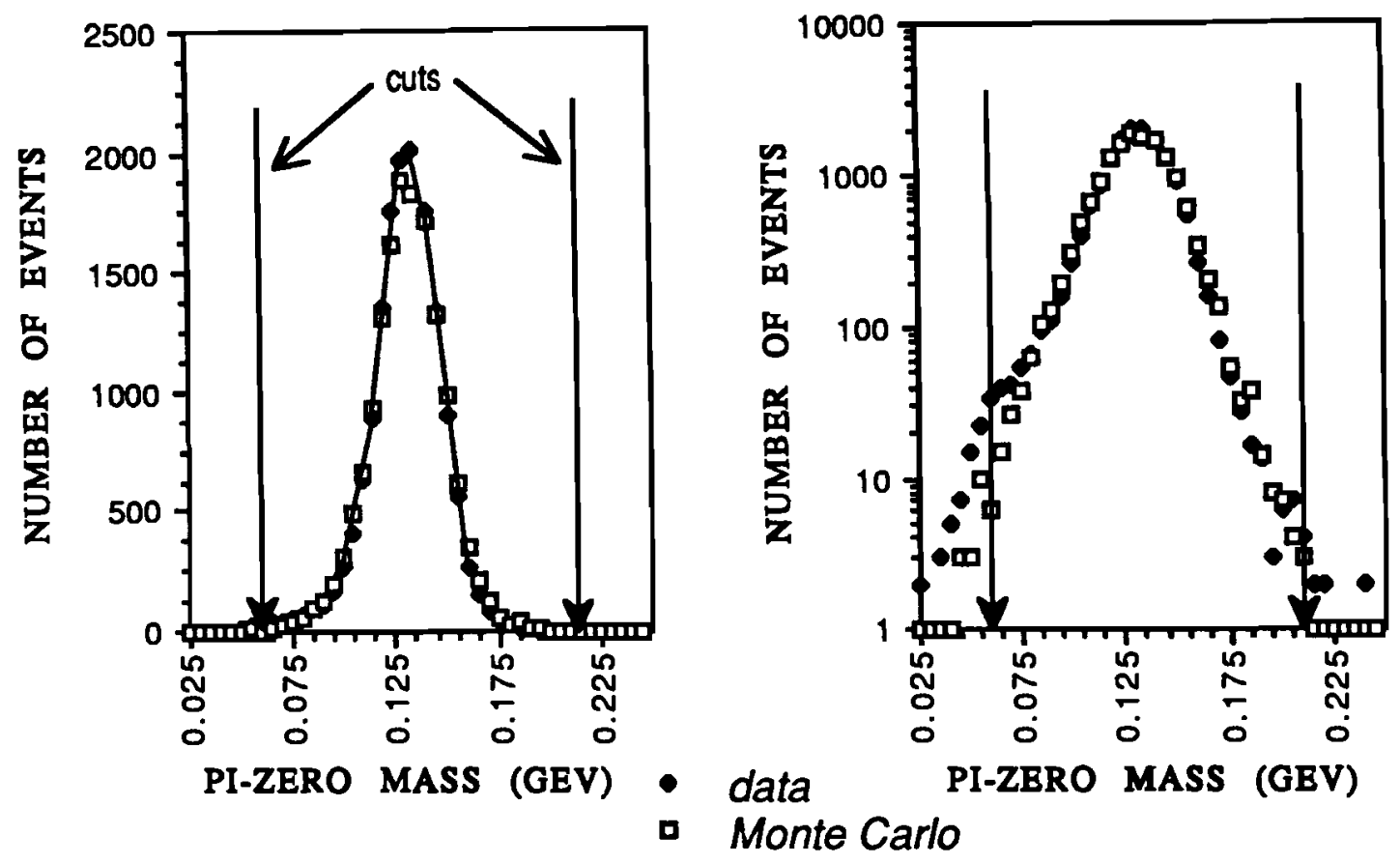

Figure 8.28 Mass of $\pi^{0}$

\subsection{3 $\mathrm{K}_{3 \pi}$ Dalitz Plot}

The $\mathrm{K}_{3 \pi}$ Dalitz plot contains information about the center of mass variables. in the $\mathrm{K}_{3 \pi}$ decay. Comparisons between Monte Carlo and data Dalitz plots may provide information about backgrounds in the $K_{3 \pi}$ data sample. Tables of $P_{c m} \pi^{+}$versus $P_{c m}$ $\pi^{-}$in $4 \mathrm{MeV}$ bins for Monte Carlo minus data were made. These "difference" Dalitz distributions between Monte Carlo and data are shown in Tables F.1-F.2, in Appendix $F$, for the upstream and downstream data. Table F.3 gives the difference between the Monte Carlo (with no signal for $\eta_{+-0}$ ) and another Monte Carlo sample with an $\eta_{+-0}$ signal of magnitude 0.14 and phase $-45^{\circ}$ in order to see how a signal might exhibit itself in the Dalitz distribution. The tables are of Monte Carlo minus data, where both distributions are normalized to 2000 events before the difference is taken.

From these distributions there is a hint of a possible background of unknown origin in both upstream and downstream data in column 10 corresponding to a $P_{\text {com }}$ $\pi^{-}$of $42 \pm 2 \mathrm{MeV}$. In hopes of improving the $z$ vertex distribution discrepancy, the these events were removed from both the data and the Monte Carlo samples for the final determination of $\eta_{+-0}$. In actuallity, this final cut had no effect on the final 
measurement of $\eta_{+-0}$ when it was removed.

\begin{tabular}{|c|c|c|}
\hline data selection criteria & $\begin{array}{c}\% \text { events } \\
\text { passed }\end{array}$ & events left \\
\hline triggers & & 100.0 \\
\hline reconstructs to a vertex & $59 \%$ & 59.0 \\
\hline K3pi candidate(charged mass) & $16 \%$ & 9.4 \\
\hline photon energies $>1.5,2.0$ & $80 \%$ & 7.5 \\
\hline charged pi-pi mass(.28-.36 GeV) & $96 \%$ & 7.2 \\
\hline slope ratio $(0.3->3.0)$ & $99 \%$ & 7.1 \\
\hline$P T^{* * 2}(<0.033)$ & $97 \%$ & 6.9 \\
\hline charged e plus-e minus mass $(>0.085)$ & $80 \%$ & 5.5 \\
\hline maximum number of wire hits $<5 /$ plane & $90 \%$ & 5.0 \\
\hline z vertex(2-19 m) & $90 \%$ & 4.5 \\
\hline kaon momentum(130-350 GeV) & $64 \%$ & 2.9 \\
\hline beam phase space requirements & $90 \%$ & 2.6 \\
\hline photon selection criteria & $80 \%$ & 2.1 \\
\hline dalitz plot requirement & $90 \%$ & 1.9 \\
\hline other cuts (mass requirements) & $97 \%$ & 1.8 \\
\hline
\end{tabular}

Table $8.4 \mathrm{~K}_{3 \pi}$ Data Losses

Table 8.4 shows all the significant $K_{3 \pi}$ data selection criteria and the percent of events which passed each. Out of 9.36 million triggers, 122 thousand events were used in the final measurement of $\eta_{+-0}$ or $1.3 \%$. All comparison plots between Monte Carlo and data in the $K_{3 \pi}$ data sample agreed very well except the $z$ of the decay vertex distribution. Figures 8.29 through 8.35 show the final $K_{3 \pi}$ distributions of 


\section{1}

the $z$ of the decay vertex, the proper time of the decay, the lambda mass, the $\mathrm{e}^{+} \mathrm{e}^{-}$ mass, the charged track opening angle, the momentum of the $\pi^{+}$and the momentum of the $\pi^{-}$. Figures 8.31 and 8.32 of the lamda and $\mathrm{e}^{+} \mathrm{e}^{-}$mass are plots of the charged particle mass assuming that the charged particles correspond to a proton and a pion or an electron and a positron respectively.

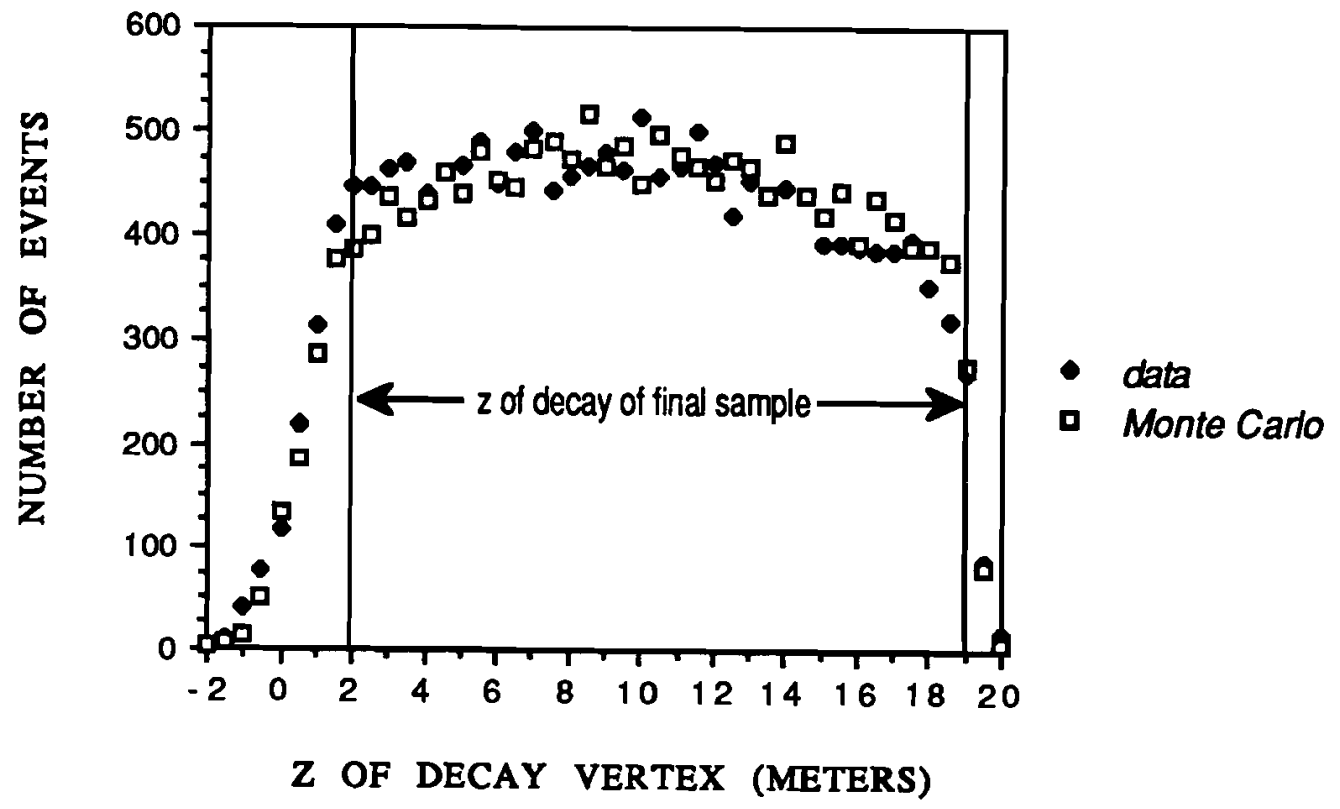

Flgure 8.29 $Z$ Vertex of $\mathbf{K}_{3 \pi}$ Decay 


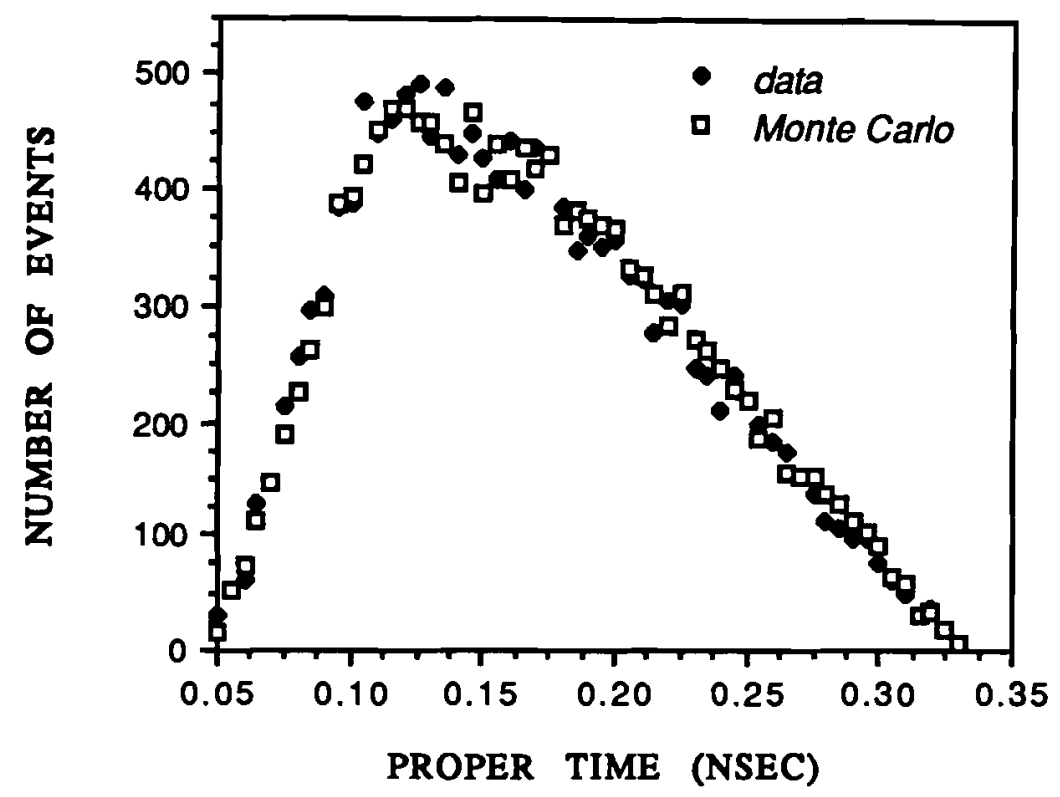

Figure 8.30 Proper Time of $\mathrm{K}_{\mathbf{3} \pi}$ Decay

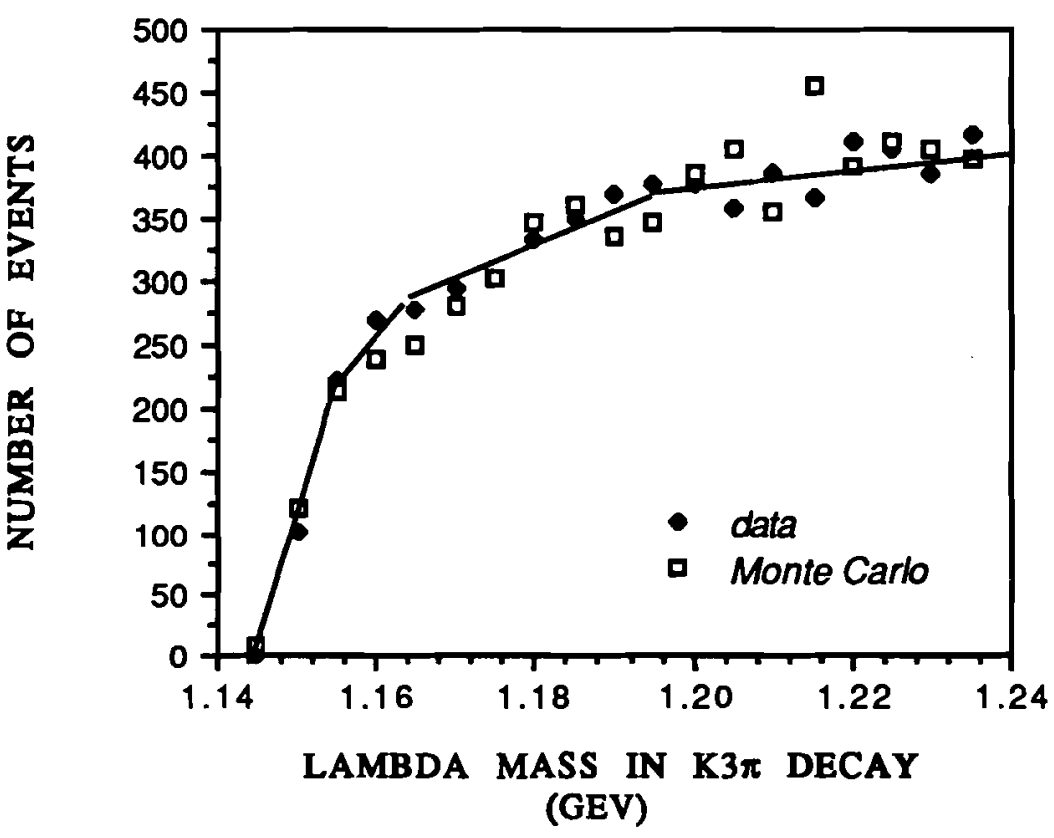

Figure 8.31 Lambda Mass In $\mathrm{K}_{3 \pi}$ Sample 
153

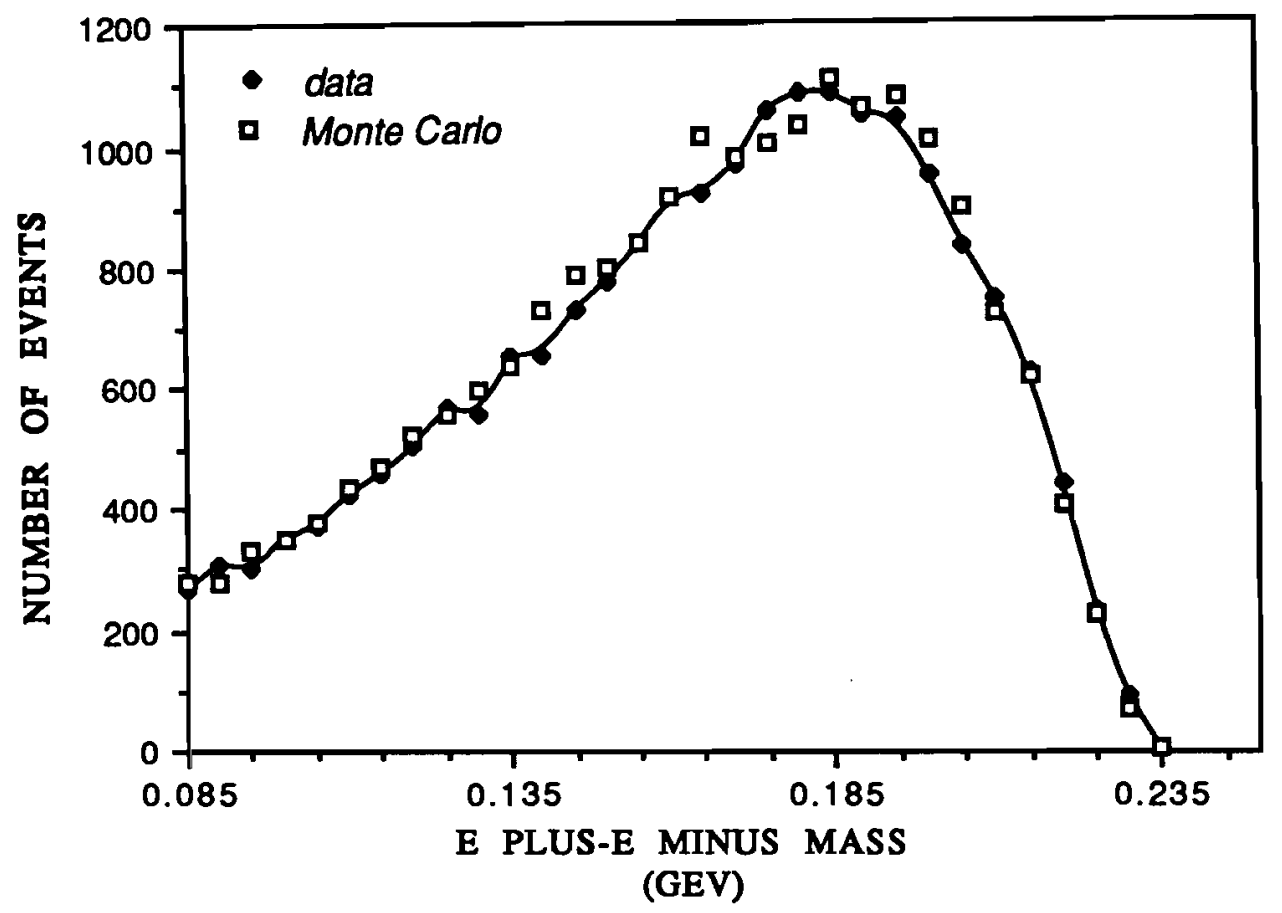

Figure 8.32 $\mathrm{e}^{+} \mathrm{e}^{-}$Mass In $\mathrm{K}_{3 \pi}$ Sample with All Cuts

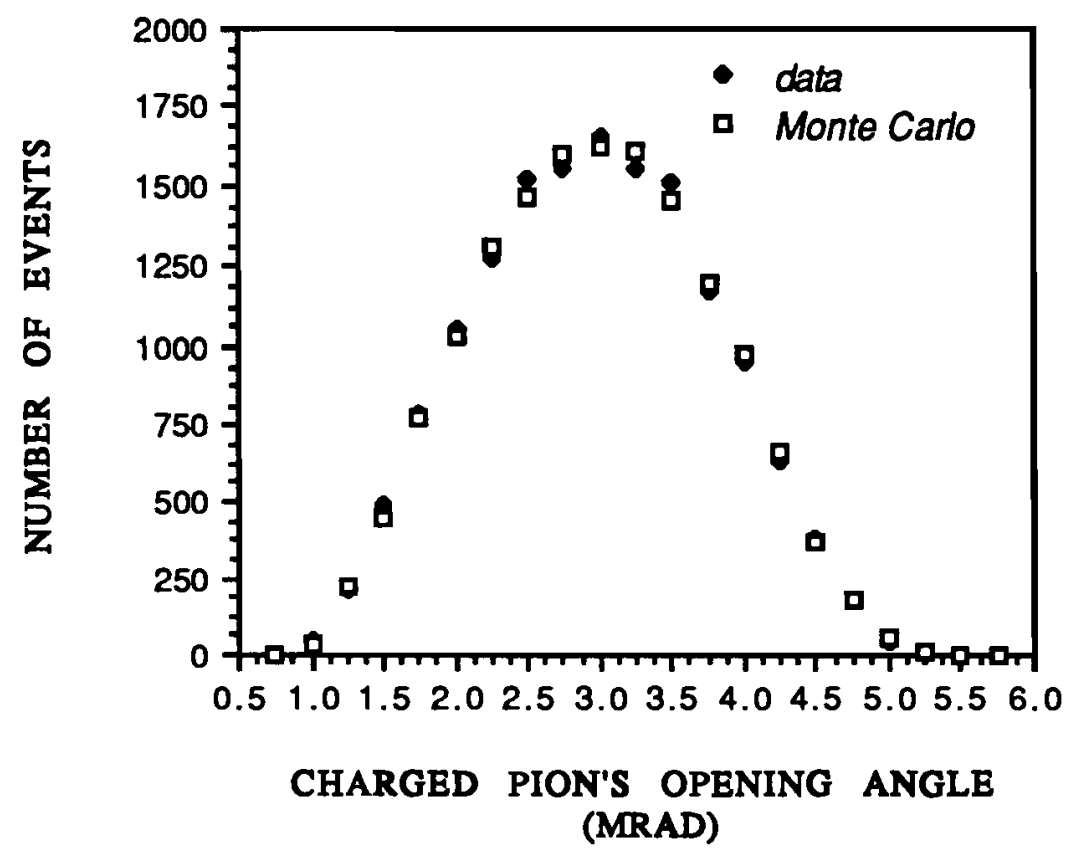

Figure 8.33 Opening Angle of the Charged Tracks In $\mathrm{K}_{3 \pi}$ Decay 


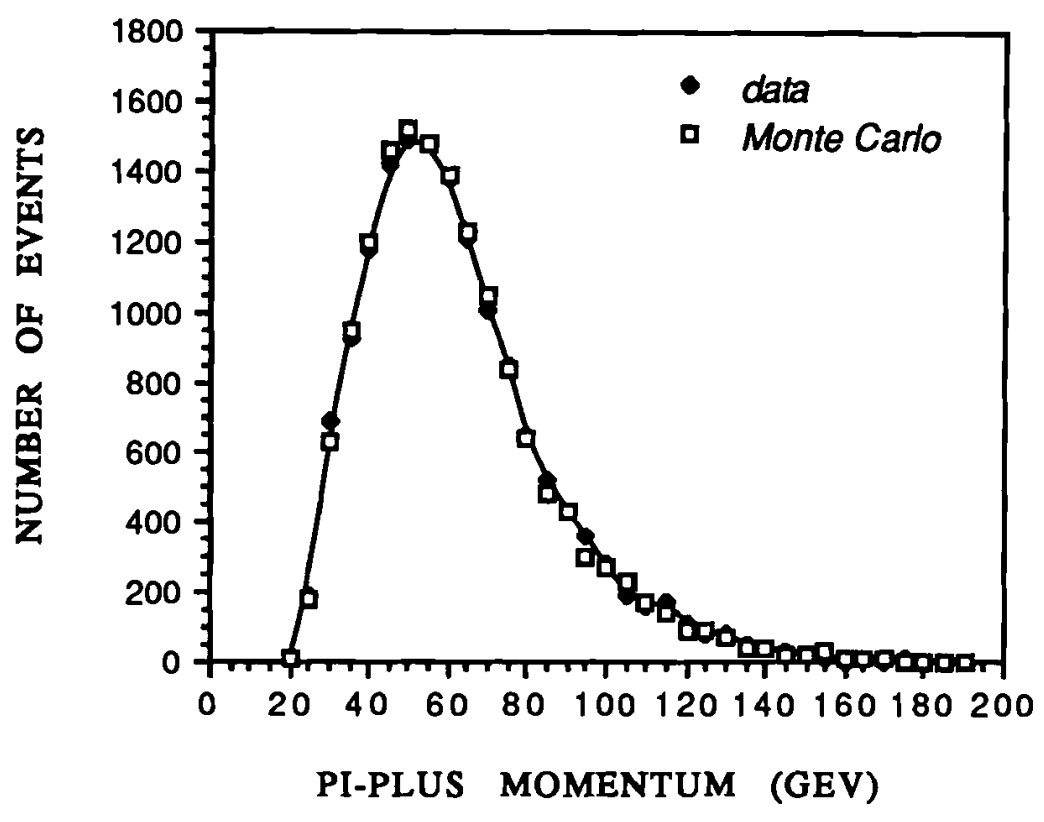

Flgure 8.34 Momentum of $\pi^{+}$in $K_{3 \pi}$ Decay

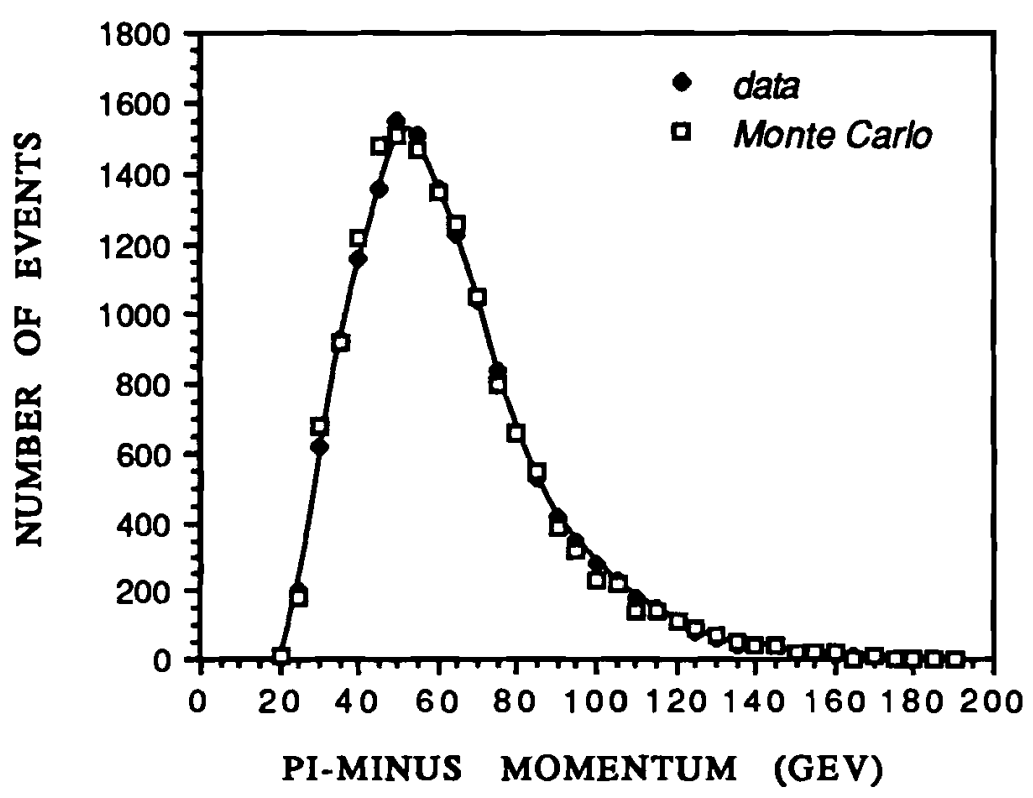

Figure 8.35 Momentum of $\pi^{-} \ln \mathrm{K}_{3 \pi}$ Decay 


\section{Chapter Nine}

\section{Physics Analysis}

\subsection{General}

The measurement of $\eta_{+-0}$ is similar to a lifetime measurement since $\eta_{+-0}$ also determines the decay probability of kaons. In this experiment one determines the number of particles of a particular type that decay over a certain region of proper time (i.e. between $t_{1}$ and $t_{2}$ ). The proper time for a particle can then be related to the more directly measurable quantities momentum (p) and decay position $(\mathrm{z})$, measured from the production target, as well as the mass of the particle by the expression $\mathrm{t}=\mathrm{zm} / \mathrm{p}$.

The final reconstructed data sample was arranged into arrays giving the number of events detected as a function of the momentum and position of the decay vertex of the kaon. The bin size for the momentum and $z$ of the decay vertex used for this analysis was $10 \mathrm{GeV}$ by $0.5 \mathrm{~m}$ respectively. This created arrays $\mathrm{N}\left(\mathrm{p}_{i}, \mathrm{z}_{j}\right)$, where $\mathrm{N}$ is the number of events that decayed in each $10 \mathrm{GeV}$ by $0.5 \mathrm{~m}$ range specified by $i$ and $j$. Then $\mathrm{N}\left(\mathrm{p}_{i}, \mathrm{z}_{j}\right)$ can be compared to $\mathrm{N}\left(\mathrm{t}_{1} \rightarrow \mathrm{t}_{2}\right)$, where $\mathrm{t}_{1} \rightarrow \mathrm{t}_{2}$ is the proper time range for the kaon decays corresponding to the bin $\left(\mathrm{p}_{i}, \mathrm{z}_{j}\right)$. The array of $\mathrm{N}\left(\mathrm{p}_{i}, \mathrm{z}_{j}\right)$ 's determined from the spectrometer for accepted decays (130 GeV to 350 $\mathrm{GeV}$ and $2.0 \mathrm{~m}$ to $19.0 \mathrm{~m}$ ), determines the distribution $N(t)$ (observed). The Monte Carlo simulation program data was arranged into the same $p$ and $z$ arrays as the data and then used to calculate the acceptance of the experiment in each $(p, z)$ bin. Comparing the experimental distribution $\mathrm{N}\left(\mathrm{p}_{i}, \mathbf{z}_{j}\right)$ to the theoretical decay distribution for kaons $N(t)$ (theory), given by equation 9.1 , when the theoretical distribution is corrected for the spectrometer acceptance, establishes a value for $\eta_{+-0}$

The theoretical distribution for the $\mathrm{K} \rightarrow 3 \pi$ decay is given by equation 1.56 in Chapter 1. Integrating this expression from proper time $t_{1}$ to $t_{2}$ yields the number 
of kaons that decay between time $t_{1}$ and time $t_{2}, N_{3 \pi}\left(t_{1} \rightarrow t_{2}\right)$ :

$$
\begin{aligned}
N_{3 \pi}\left(t_{1} \rightarrow t_{2}\right) & =N_{K}(0) n_{3 \pi}\left(\eta_{+-o}, t_{1}, t_{2}\right) \\
N_{3 \pi}\left(t_{1} \rightarrow t_{2}\right)= & \frac{N_{K}(0)}{C_{3 \pi}} \tau_{\ell}\left[e^{-t_{1} / \tau_{\ell}}-e^{-t_{2} / \tau_{\ell}}\right]+\frac{\left|\eta_{+-o}\right| \mathbf{D}_{K \bar{K}}}{\frac{1}{4 \tau_{s}^{2}}+(\Delta m)^{2}} * \\
& *\left\{e^{-t_{1} / 2 \tau_{\mathrm{s}}}\left[\frac{1}{\tau_{s}} \cos \left(\Delta m t_{1}-\varphi_{+-o}\right)-2 \Delta m \sin \left(\Delta m t_{1}-\varphi_{+-o}\right)\right]+\right. \\
& \left.+e^{-t_{2} / 2 \tau_{\mathrm{s}}}\left[2 \Delta m \sin \left(\Delta m t_{2}-\varphi_{+-o}\right)-\frac{1}{\tau_{s}} \cos \left(\Delta m t_{2}-\varphi_{+-o}\right)\right]\right\} \\
C_{3 \pi}= & +\frac{\mid \eta_{\ell}}{\frac{1}{4 \tau_{s}^{2}}+(\Delta m)^{2}}\left[\frac{1}{\tau_{s}} \cos \varphi_{+-o}+2 \Delta m \sin \varphi_{+-o}\right)
\end{aligned}
$$

To convert $\mathrm{N}_{3 \pi}(\mathrm{t})$ of equation 9.1 to $\mathrm{p}$ and $\mathrm{z}$, one approximates:

$$
t_{1}=\frac{z_{j \min } m_{K}}{p_{c i}} \text { and } t_{2}=\frac{z_{j \max } m_{K}}{p_{c i}}
$$

where $m_{\mathrm{K}}$ is the kaon mass, $\mathrm{p}_{\mathrm{c} i}$ the kaon momentum at the center of the $10 \mathrm{GeV}$ wide bin $\mathrm{p}_{i}$ and $\mathrm{z}_{j \min }$ and $\mathrm{z}_{j \max }$ are the end points of the 0.5 meter wide $\mathrm{z}$ bin $\mathrm{z}_{j}$. These expressions for $\mathrm{t}_{1}$ and $\mathrm{t}_{2}$ were substituted into equation 9.1 to obtain $\mathrm{N}\left(\mathrm{p}_{i}, \mathrm{z}_{j}\right)$. The $\mathrm{p}$ and $z$ arrays were 22 bins by 34 bins respectively, for $p$ ranging from $130 \mathrm{GeV}$ to $350 \mathrm{GeV}$ and $z$ ranging form $2.0 \mathrm{~m}$ to $19.0 \mathrm{~m}$. N $\left(\mathrm{p}_{i}, \mathrm{z}_{j}\right)$ was calculated for each bin by fixing $\mathrm{p}_{c i}$ to the central value of the bin $i$ and integrating from $z_{j \min }$ to $z_{j \max }$.

$$
N_{3 \pi}\left(p_{i}, z_{j}\right)=N_{K}(0) n_{3 \pi}\left(\eta_{+-o}, p_{c i}, z_{j}\right)
$$

In equation $9.3 n_{3 \pi}\left(\mathrm{p}_{\mathrm{c}_{i}}, \mathrm{z}_{j}\right)$ is defined by equation 9.1 with $\mathrm{t}_{1}$ and $\mathrm{t}_{2}$ from equation 9.2 and corresponds to the number of kaons of momentum $\mathrm{p}_{c_{i}}$ which decay from $z_{j \min }$ to $z_{j \max }$.

The expected array of data events, $\mathrm{N}^{\mathrm{E}}\left(\mathrm{p}_{i}, \mathrm{z}_{j}\right)$, reflects not only the decay 
probability of equation 9.1 , but also the acceptance, $A\left(\mathrm{p}_{i}, \mathrm{z}_{j}\right)$, of the spectrometer and reconstruction program. For a given $\left(\mathrm{p}_{i}, \mathrm{z}_{j}\right)$ bin one has:

$$
\begin{aligned}
& N_{3 \pi}^{\mathrm{E}}\left(p_{i}, z_{j}\right)=A_{3 \pi}^{D}\left(p_{i}, z_{j}\right) n_{3 \pi}\left(\eta_{+-o}, p_{c i}, z_{j}\right) N_{K}^{D}(0) B R_{3 \pi} \\
& N_{3 \pi}^{M C}\left(p_{i}, z_{j}\right)=A_{3 \pi}^{M C}\left(p_{i}, z_{j}\right) n_{3 \pi}\left(\eta_{+-o}^{o}, p_{c i}, z_{j}\right) N_{K}^{M C}(0)
\end{aligned}
$$

where $D$ corresponds to data and $M C$ to Monte Carlo. No branching ratio for $K \rightarrow 3 \pi$ decay is necessary in $N_{3 \pi}^{M C}\left(p_{i}, z_{j}\right)$ since all of the Monte Carlo generated kaons decay to $3 \pi$. The normalization $N_{K}^{M C}(0)$ is the number of Monte Carlo events generated at the target. $\eta_{+-o}^{o}$ is the value of $\eta_{+-0}$ used in the Monte Carlo program and $N_{3 \pi}^{M C}\left(p_{i}, z_{j}\right)$ are the resultant Monte Carlo events binned in $\mathrm{p}$ and $\mathrm{z}$. As long as the Monte Carlo's acceptance exactly matches that of the real data events, equation 9.5 can be solved for $A_{3 \pi}^{M C}\left(p_{i}, z_{j}\right)$ and this value substituted into equation 9.4 for $A_{3 \pi}^{D}\left(p_{i}, z_{j}\right)$. The expected number of events in a given bin $\left(\mathrm{p}_{i}, \mathrm{z}_{j}\right)$ is then:

$$
N_{3 \pi}^{\mathrm{E}}\left(p_{i}, z_{j}\right)=\frac{n_{3 \pi}\left(\eta_{+-o}, p_{c i}, z_{j}\right) N_{K}^{D}(0) B R_{3 \pi} N_{3 \pi}^{M C}\left(p_{i}, z_{j}\right)}{n_{3 \pi}\left(\eta_{+-o}^{o}, p_{c i}, z_{j}\right) N_{K}^{M C}(0)}
$$

The acceptance is obtained from the Monte Carlo, the branching ratio is known, and $n_{3 \pi}\left(\mathrm{p}_{\mathrm{c} i}, \mathrm{z}_{j}\right)$ is known except for $\left|\eta_{+-0}\right|$ and $\varphi_{+-0}$ which we are trying to determine.

$$
\begin{gathered}
\left|\eta_{+-o}\right|=\sqrt{\operatorname{Re}\left\{\eta_{+-o}\right\}^{2}+\operatorname{Im}\left\{\eta_{+-o}\right\}^{2}} \\
\tan \varphi_{+-o}=\frac{\operatorname{Re}\left\{\eta_{+-o}\right\}}{\operatorname{Im}\left\{\eta_{+-o}\right\}}
\end{gathered}
$$

The normalization $\mathrm{N}_{\mathrm{K}}(0)$, the number of $\mathrm{K}^{0}$ 's produced at the target, is determined from the $K \rightarrow 2 \pi$ data sample by using the same analysis applied to $K \rightarrow 2 \pi$ decay if one exchanges $\tau_{\ell} \leftrightarrow \tau_{s},\left|\eta_{+-0}\right| \rightarrow\left|\eta_{+-}\right|$and $\varphi_{+-0} \rightarrow-\varphi_{+-}$. For $K \rightarrow 2 \pi$ decay $\mid \eta_{+-}$ I and $\varphi_{+-}$are well measured and one fits the data to determine $N_{K}(0)$. Over the range of proper time used in our measurement of $\eta_{+-0}$, the real part of $\eta_{+-0}$ is $80 \%$ 
correlated with the normalization $\mathrm{N}_{\mathrm{K}}(0)$. $\eta_{+-0}$ was determined by comparing the data distribution binned in $\left(\mathrm{p}_{i}, \mathrm{z}_{j}\right)$ with equation 9.6.

Two different types of fitting techniques were used to determine $\eta_{+-0}$. One method used $\chi^{2}$ minimization and assumes Gaussian statistics and the other used the maximum likelihood method with Poisson statistics. The $\chi^{2}$ method is easier to formulate but the maximum likelihood method is more flexible. The two methods provided the same results in most cases. All results were determined using the minimization program MINUIT(42).

\section{$9.2 \chi^{2}$ Minimization}

$\chi^{2}$ minimization is a standard fitting technique where one minimizes the difference between an experimental distribution and a theoretical distribution assuming Gaussian statistics. $\chi^{2}$ measures the merit of the final result. . One minimizes:

$$
\chi^{2}\left(\eta_{+-0}\right)=\sum_{i j} \frac{\left[N_{3 \pi}^{D}\left(p_{i}, z_{j}\right)-N_{3 \pi}^{E}\left(p_{i}, z_{j}\right)\right]^{2}}{\sigma_{i j}^{2}}
$$

where $\mathrm{N}^{\mathrm{D}}\left(\mathrm{p}_{i}, \mathrm{z}_{j}\right)$ is the number of data events (the experimental value) in bin $i j$ and $N^{E}\left(p_{i}, z_{j}\right)$ is the expected number of events in bin $i j$ from equation 9.6 for $K \rightarrow 3 \pi$ decays. For $\mathrm{K} \rightarrow 2 \pi$ decays similar equations apply with the exchanges mentioned previously. The variance, $\sigma_{i j}{ }^{2}$, was assumed to be Gaussian for bins with more than 20 events i.e. $\sigma_{i j}=\sqrt{N^{D}\left(p_{i}, z_{j}\right)}$. Otherwise the uncertainty due to Poisson statistics was approximated(43) by letting $\sigma=1.9$ if $\mathrm{N}^{\mathrm{D}}=0$ and $\sigma=1.3+\sqrt{\mathrm{N}^{\mathrm{D}}}$ for $\mathrm{N}^{\mathrm{D}} \leq 20$ but not equal to zero.

\subsection{Maximum Likelihood}

Maximum likelihood is a more general method for determiring a distribution which corresponds to a data sample because it allows you to choose the appropriate probability function for the data sample. Since many bins in the $\mathrm{K}_{3 \pi}$ data sample have less than 20 events in them, the Poisson distribution is appropriate. For 
Poisson statistics the probability of receiving $D_{i j}$ events in the $i j$ bin is:

$$
P_{i j}\left(T_{i j}, D_{i j}\right)=\frac{e^{-T_{i j}} T_{i j}-D_{i j}}{D_{i j} !}
$$

where $\mathrm{T}_{i j}$ is the theoretical (expected) value and $\mathrm{D}_{i j}$ is the experimental (data) value. The probabily of receiving the data in all bins is the product of the probability of the individual bins.

$$
\mathcal{P}(p, z)=\prod_{i j} P_{i j}\left(T\left(p_{i}, z_{j}\right), D\left(p_{i}, z_{j}\right)\right)
$$

The parameters can then be varied to maximize $\mathcal{P}$. For comparison to the $\chi^{2}$ method one forms:

$$
\mathcal{L}(p, z)=-2 \ln \left(\frac{\boldsymbol{P}\left(p_{i}, z_{j}\right)}{\boldsymbol{P}_{\mathrm{o}}\left(p_{i}, z_{j}\right)}\right)
$$

where $\boldsymbol{P}_{0}$ is the maximum likelihood function evaluated at $\mathrm{T}=\mathrm{D}$, where theory and data match. $\mathcal{L}(\mathrm{p}, \mathrm{z})$ is then equivalent to a $\chi^{2}$ for samples where Gaussian statistics are valid. Substituting equations 9.10 and 9.11 into 9.12 one obtains:

$$
\mathcal{L}(p, z)=2 \sum_{i j}\left[\left(T_{i j}-D_{i j}\right)-D_{i j} \ln \left(\frac{T_{i j}}{D_{i j}}\right)\right]
$$

where the sums over $i$ and $j$ are over the bins $\left(\mathrm{p}_{i}, \mathrm{z}_{j}\right)$.

In the limit where $\mathrm{D}_{i j} \rightarrow 0$ equation 9.13 approaches $2 \mathrm{~T}_{i j}$, but if $\mathrm{T}_{i j}$ is zero it is infinite. $\mathrm{T}_{i j}$ is the theoretical value from equation 9.6 and thus as long as the Monte Carlo statistics are large enough for the $\mathrm{p}$ and $\mathrm{z}$ bins, the method is valid.

\subsection{K $\rightarrow 2 \pi$ Measurements}

The $\mathrm{K} \rightarrow 2 \pi$ decay parameters $\tau_{\ell}, \tau_{s}, \Delta m, \eta_{+-}$and $\varphi_{+_{-}}$of the decay equation $n\left(\eta_{+-}, \mathrm{p}, \mathrm{z}\right)$ are all well measured $(41)$. This fact was used to verify the analysis 
160

procedure by measuring the $\mathrm{K}_{\mathrm{s}}$ lifetime. This also verified a large portion of the $\mathrm{K} \rightarrow 3 \pi$ acceptance calculation as the two decays used all common routines for the charged portion of the decay.

\subsection{1 $\mathrm{K}_{\mathrm{s}}$ Lifetime}

The expected number of events in a given $\mathrm{p}$ and $\mathrm{z}$ bin is derived in the same manner as for $\mathrm{K} \rightarrow 3 \pi$ decay. Similar to equation 9.6 one has:

$$
N_{2 \pi}^{E}\left(p_{i}, z_{j}\right)=\frac{n_{2 \pi}\left(\eta_{+-}, p_{c i}, z_{j}\right) N_{K}^{D}(0) B R_{2 \pi} N_{2 \pi}^{M C}\left(p_{i}, z_{j}\right)}{n_{2 \pi}\left(\eta_{+-}^{o}, p_{c i}, z_{j}\right) N_{K}^{M C}(0)}
$$

With $n_{2 \pi}\left(\mathrm{p}_{\mathrm{ci}}, \mathbf{z}_{j}\right)$ defined by:

$$
t_{1}=\frac{z_{j \min } m_{K}}{p_{c i}} \text { and } t_{2}=\frac{z_{j \max } m_{K}}{p_{c i}}
$$

substituted into:

$$
\begin{aligned}
n_{2 \pi}\left(t_{1}, t_{2}\right)= & \frac{1}{C_{2 \pi}} \tau_{\mathrm{s}}\left[e^{-t_{1} / \tau_{\mathrm{s}}}-e^{-t_{2} / \tau_{\mathrm{s}}}\right]+\frac{\left|\eta_{+-}\right| \mathrm{D}_{K \bar{K}}}{1 / 4 \tau_{s}^{2}+\Delta m^{2}} * \\
& *\left\{e^{-t_{1} / 2 \tau_{\mathrm{s}}}\left[\tau_{\mathrm{s}} \cos \left(\Delta m t_{1}+\varphi_{+-}\right)-2 \Delta m \sin \left(\Delta m t_{1}+\varphi_{+-}\right)\right]+\right. \\
& \left.+e^{-t_{2} / 2 \tau_{\mathrm{s}}}\left[2 \Delta m \sin \left(\Delta m t_{2}+\varphi_{+-}\right)-\tau_{s} \cos \left(\Delta m t_{2}+\varphi_{+-}\right)\right]\right\} \\
C_{2 \pi}= & \tau_{\mathrm{s}}+\frac{\left|\eta_{+-}\right| \mathrm{D}_{K \bar{K}}}{1 / 4 \tau_{s}^{2}+\Delta m^{2}}\left(\tau_{s} \cos \varphi_{+-}-2 \Delta m \sin \varphi_{+-}\right)
\end{aligned}
$$

All the variables in equation 9.14 are known except $N_{K}^{D}(0)$, the number of kaons produced in the target. In determining the lifetime $\tau_{\mathrm{s}}$ and $N_{K}^{D}(0)$ were determined by the fit since the normalization was not a priori known.

As a test, a Monte Carlo sample was generated with a known lifetime and then the lifetime was measured using the analysis technique outlined above. Both the $\chi^{2}$ and the maximum likelihood method gave the correct result and it was determined that no momentum dependence was introduced by the lifetime analysis.

The results for the data using both the $\chi^{2}$ method of equation 9.9 and the maximum likelihood method of equation 9.13 are given in Table 9.1. The uncertainties are purely statistical. The data were fit over a momentum region from 100 to $350 \mathrm{GeV}$ and a decay vertex region from 2 to 19 meters. The results are 
given for the downstream east and downstream west targets only. The lifetime could not be well measured from the upstream data alone because of limited statistics, but a result for all four targets is given. The upstream targets were over $5 \mathrm{~K}_{\mathrm{s}}$ lifetimes upstream of the decay region. The data sample consisted of $212 \mathrm{~K}$ and $186 \mathrm{~K}$ events from the downstream east and downstream west targets respectively. Monte Carlo statistics of approximately 10 times that of the data were used in determining the values of Table 9.1. The dilution factor, $\mathbf{D}_{\mathbf{K} \overline{\mathbf{K}}}$, in the Monte Carlo and fitting program was fixed at 0.5 for all fits, a reasonable value as explained in Appendix D. Of course this measurement of the $K_{s}$ lifetime is not really sensitive to any of the actual values of the CP symmetry violation parameters. The $\chi^{2 / D O F}$ is determined by finding the $\chi^{2}$ using equation 9.9 and dividing it by the number of degrees of freedom in the fit which corresponds to the number of $p$ and $z$ bins minus one (or $i j$ 1 in equation in equation 9.9). The number of degrees of freedom in the fit for the momentum range used in measuring the lifetime, $100-350 \mathrm{GeV}$, is 849 .

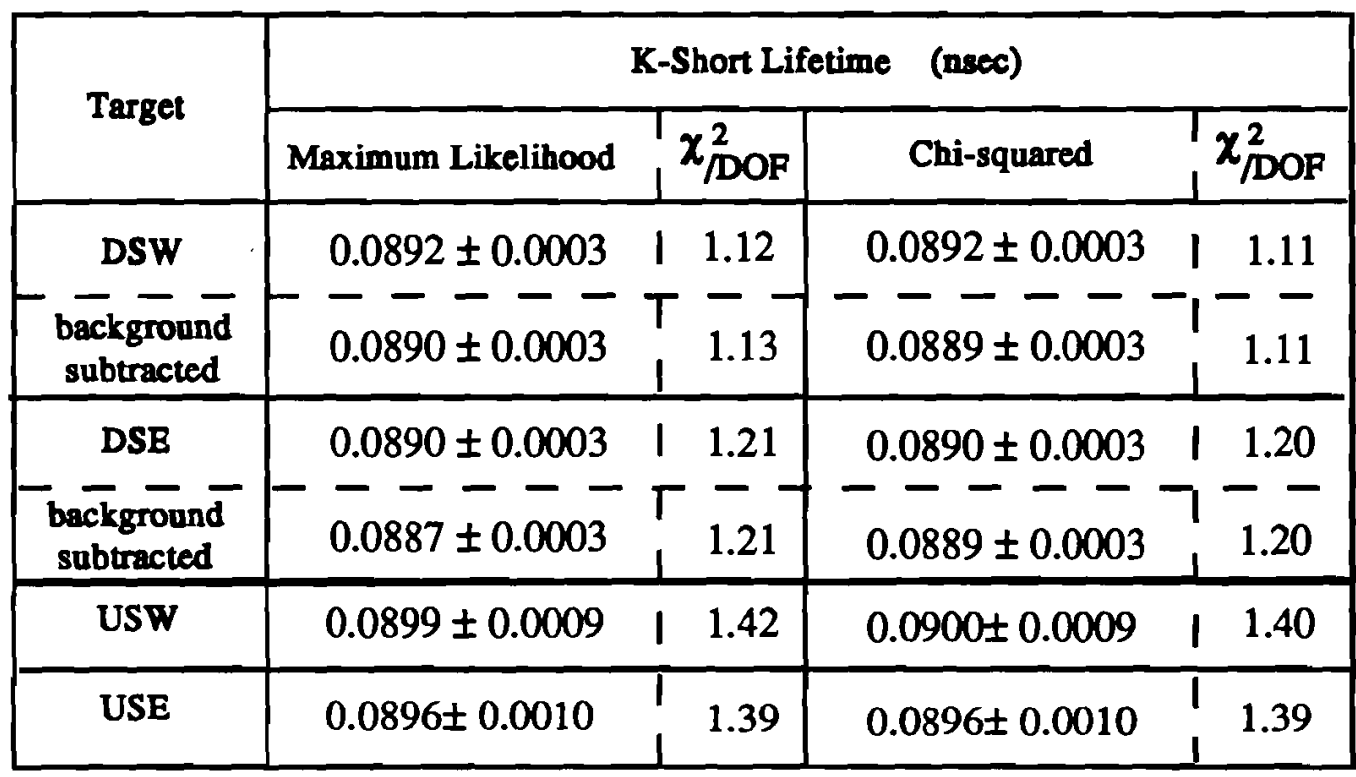

Table 9.1 K, Lifetlme for All Targets

The background of collimator produced $\mathrm{K} \rightarrow 2 \pi$ decays was found to be $\sim 0.5 \%$ for the downstream east target and $\sim 0.9 \%$ for the downstream west target and was simulated as described in Appendix C. Simulated collimator produced events (more 
than 10 times the calculated background statistics) were normalized to the calculated data background level and used to subtract the background from each $p$ and $z$ bin. The lifetime measured with these corrected data samples is shown in Table 9.1. The measured lifetime is in excellent agreement with the world average of $0.0892 \pm 0.0002$ nsec. The measured lifetimes for the two upstream targets is only shown with the collimator produced background subtracted.

As a further test of possible systematic uncertainties, I measured the $K_{s}$ lifetime as a function of kaon momentum in $50 \mathrm{GeV}$ momentum bins. The results are shown in Figure 9.1 where the collimator produced background has been subtracted from the data sample. No significant momentum dependence of the lifetime was observed. Using all four target data samples with collimator production subtracted, I obtained a lifetime of $0.0889 \pm 0.0002$ nsec with a $\chi^{2} /$ DOF of 1.12 .

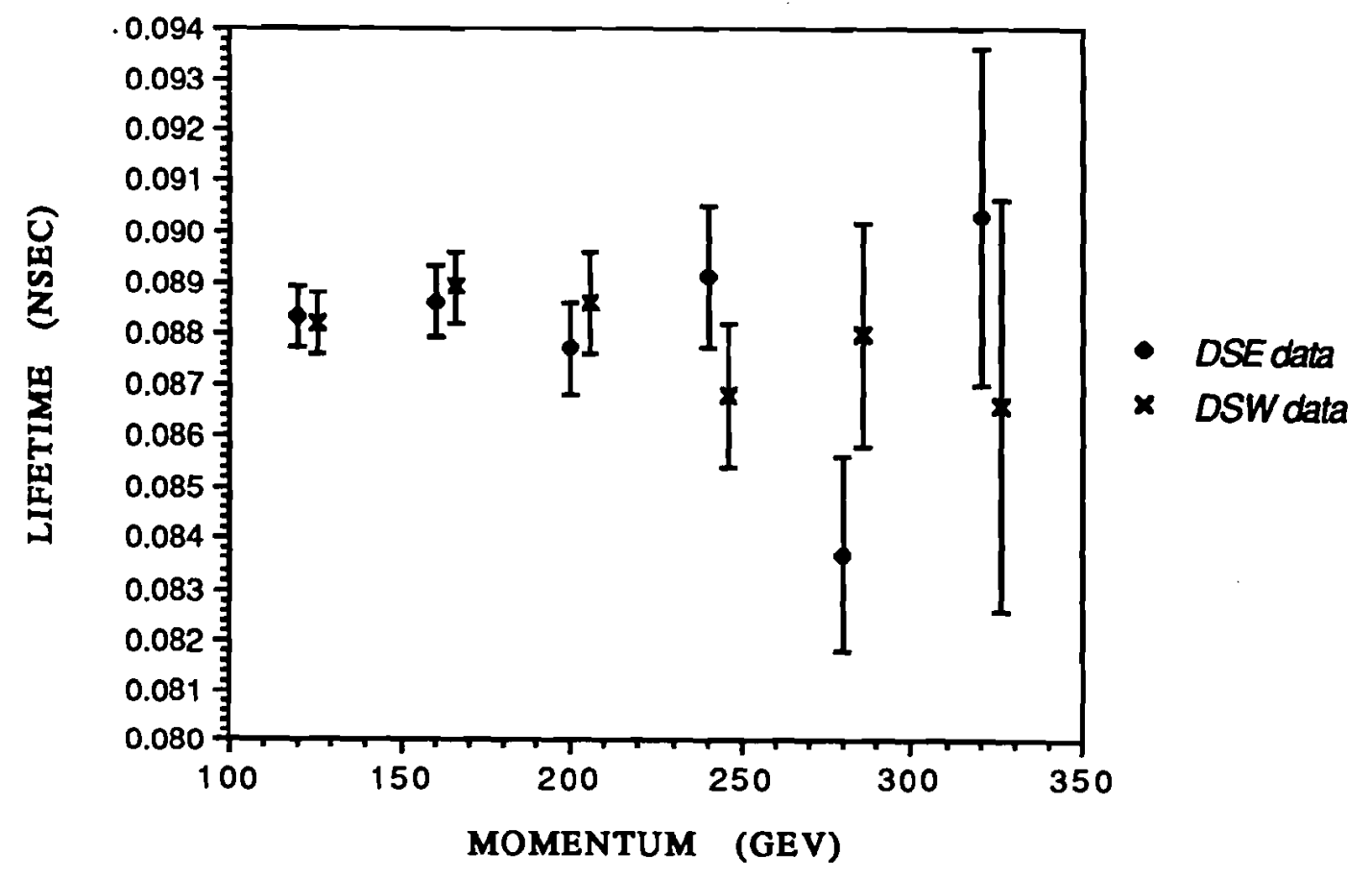

Figure $9.1 \mathrm{~K}$, Llfetlme v8. Momentum

\subsubsection{Normalization Values of Kaons on Target}

The $\mathrm{K} \rightarrow 2 \pi$ sample was mainly needed to measure $N_{K}^{D}(0)$, the number of kaons produced at the target since $\eta_{+-0}$ is highly correlated to $N_{K}^{D}(0)$. To determine this normalization, the decay positon was restricted to the range of $2 \mathrm{~m}$ to $19 \mathrm{~m}$ from the 
downstream face of magnet M1 with a momentum from $130 \mathrm{GeV}$ to $350 \mathrm{GeV}$. The lifetime, $\tau_{s}$, and all other parameters in equation 9.16 were fixed $^{(41)}$ to determine $N_{K}^{D}(0)$ for each of the four targets independently. A summary of the results is given in Table 9.2. The number of data events used in determining these normalization values was 181,000 and 159,000 for the downstream east and west targets respectively and 7282 and 9427 for the upstream east and west targets respectively. The number of degrees of freedom in the fit for the momentum range used in measuring the normalization values, $130-350 \mathrm{GeV}$, was 747.

\begin{tabular}{|c|c|c|}
\hline \multirow{2}{*}{ BEAM } & \multicolumn{2}{|c|}{$\begin{array}{c}\text { Nomalization of the Number of Kaons Produced on the Target } \\
\text { using Maximum Likelihood Method }\end{array}$} \\
\hline & DOWNSTREAM $\boldsymbol{x}_{\text {DOF }}^{2}$ & UPSTREAM \\
\hline $\begin{array}{c}\text { WEST BEAM } \\
\begin{array}{c}\text { background } \\
\text { subtracted }\end{array}\end{array}$ & 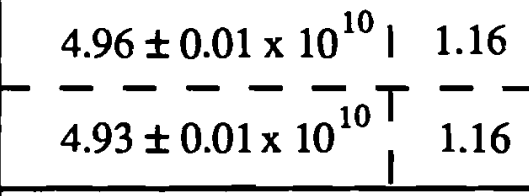 & $\begin{array}{r}2.74 \pm 0.03 \times 10^{10} \mid \\
-\overline{7}-\overline{1} \\
2.72 \pm 0.03 \times 10^{10} \mid\end{array}$ \\
\hline $\begin{array}{l}\text { EAST BEAM } \\
\text { background } \\
\text { subtracted }\end{array}$ & $\begin{array}{ll}5.35 \pm 0.01 \times 10^{10} & 1.17 \\
5.32 \pm 0.01 \times 10^{10} & \frac{1}{1.17}\end{array}$ & $\begin{array}{l}2.16 \pm 0.03 \times 10^{10} \mid 1.37 \\
2.15 \pm 0.03 \times 10^{10} \mid-\frac{1.37}{-}\end{array}$ \\
\hline
\end{tabular}

Table 9.2 Total Number of Kaons

Collimator produced $\mathrm{K} \rightarrow 2 \pi$ background was small, but $\mathrm{I}$ did correct for it to obtain a more accurate normalization. The upstream west sample had the largest background, $-1.5 \%$, the smallest was the two east samples at $0.4 \%$. The effects of background subtraction changed the upstream normalization by less than one standard deviation and 2-3 standard deviations downstream as can be seen in Table 9.2. The normalization values were consistent between the two fitting methods, $\chi^{2}$ minimization and maximum likelihood.

The $\mathrm{K} \rightarrow 2 \pi$ sample had little or no background and most of the selection criteria applied to the sample had little effect. To test for systematic uncertainties, I remeasured the normalization after loosening the mass constraint of Figure 8.10 by $0.01 \mathrm{GeV}$ and tightening the beam phase space requirements of Table 8.2 by $10 \%$. The $\mathrm{K}_{2 \pi}$ mass and neutral beam phase space requirements were the most significant 
constraints applied to the $\mathrm{K} \rightarrow 2 \pi$ data sample. The results of these systematic uncertainty checks are shown in Table 9.3.

Only the possible systematic uncertainty on the downstream east target normalization was larger than the statistical uncertainty. Thus, in the $\mathrm{K}_{3 \pi}$ analysis I used the statistical uncertainty for the normalization for all targets except the downstream east target. For the downstream east target I used the systematic uncertainty of 0.04 .

\begin{tabular}{|c|c|c|c|c|}
\hline \multirow{2}{*}{ BEAM } & \multicolumn{4}{|c|}{$\begin{array}{c}\text { Nomalization of the Number of Kaons Produced on the Target } \\
\text { using Maximum Likelihood Method- Systematic Errors } \\
(\times 10)^{0}\end{array}$} \\
\cline { 2 - 5 } & Original & $\begin{array}{c}\text { Looser K2 Mass } \\
\text { Constraint }\end{array}$ & $\begin{array}{c}10 \% \text { Tighter Beam } \\
\text { Constraint }\end{array}$ & $\begin{array}{c}\text { Possible Systematio } \\
\text { Uncertainty }\end{array}$ \\
\hline $\begin{array}{c}\text { Downstream } \\
\text { West Beam }\end{array}$ & $4.93 \pm 0.01$ & $4.94 \pm 0.01$ & $4.92 \pm 0.01$ & 0.01 \\
\hline $\begin{array}{c}\text { Upstream } \\
\text { West Beam }\end{array}$ & $2.72 \pm 0.03$ & $2.73 \pm 0.03$ & $2.71 \pm 0.03$ & 0.01 \\
\hline $\begin{array}{c}\text { Downstream } \\
\text { East Beam }\end{array}$ & $5.32 \pm 0.01$ & $5.33 \pm 0.01$ & $5.28 \pm 0.01$ & 0.04 \\
\hline $\begin{array}{c}\text { Upstream } \\
\text { East Beam }\end{array}$ & $2.15 \pm 0.03$ & $2.15 \pm 0.03$ & $2.14 \pm 0.03$ & 0.01 \\
\hline
\end{tabular}

Table 9.3 Systematlc Uncertainty in the Normallzation of the Number of Kaons

\subsection{The $\eta_{+-0}$ Measurement}

If the acceptance of the apparatus were perfectly understood, each of the four data samples, downstream west (DSW), downstream east (DSE), upstream west (USW), and upstream east (USE), could be analyzed seperately. Any inconsistency, which we call a bias, in these results indicates a lack of knowledge about the behavior of the apparatus. One can then use the different data samples to correct this bias. Such a bias in the data was discovered. Its existence is suggested by the discrepancy in the Monte Carlo and data comparison plots of $z$ vertex in Figure 9.2. Figure 9.2 shows the comparison of the $z$ of the decay vertex for Monte Carlo and data for $K \rightarrow 3 \pi$ decay. There is a slight excess of data events in the upstream region. 


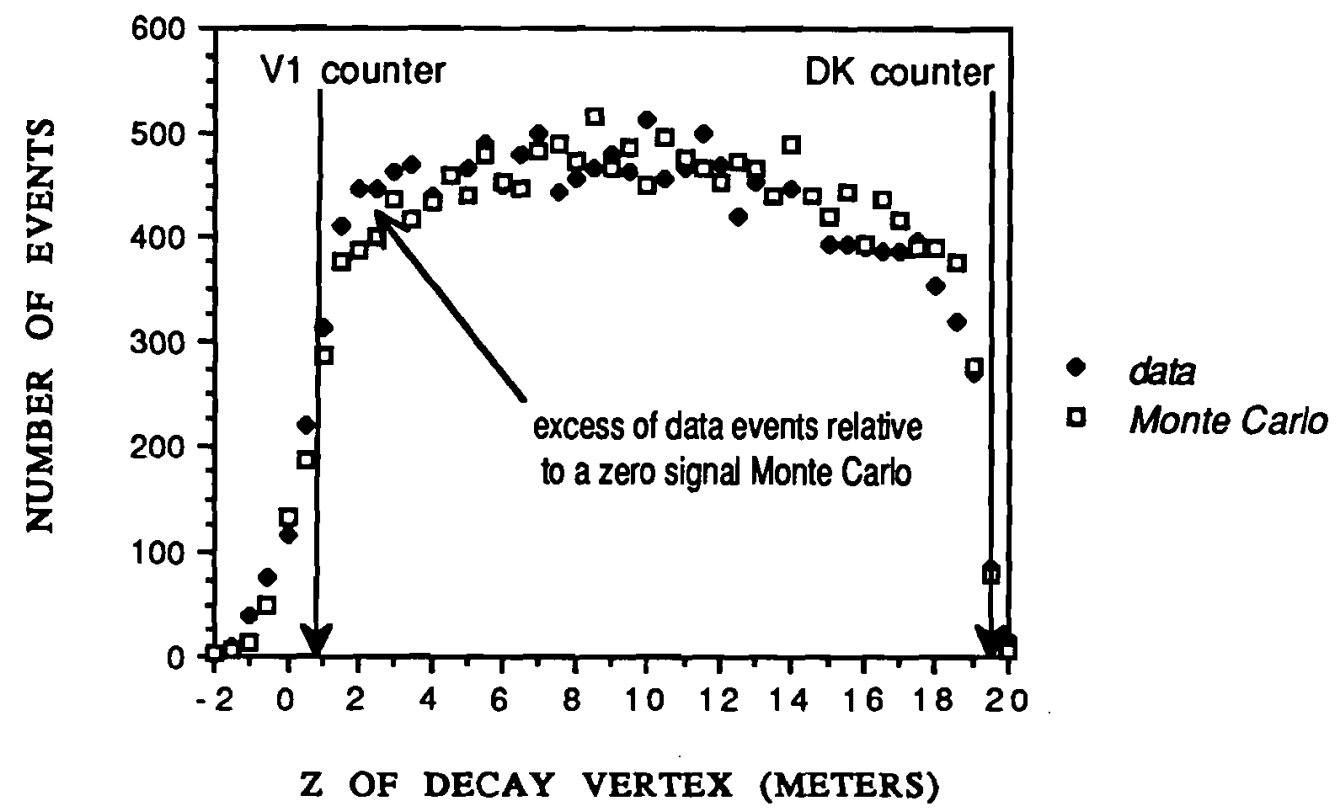

Figure 9.2 $\mathrm{K}_{3 \pi}$ Z-of Decay Vertex

The final $\mathrm{K} \rightarrow 3 \pi$ data statistics were:

$\begin{array}{ll}\text { downstream west data } & 43 \mathrm{~K} \\ \text { downstream east data } & 39 \mathrm{~K} \\ \text { upstream west data } & 21 \mathrm{~K} \\ \text { upstream east data } & 19 \mathrm{~K}\end{array}$

In the all data calculations the effect of collimator produced kaon decays, calculated to be $<1.5 \%$ for each target, is removed by subtracting a set of simulated Monte Carlo background events (see Appendix $\mathrm{C}$ ). The momentum and $\mathrm{z}$ of the decay ranges used in all measurements were 130 to $350 \mathrm{GeV}$ and 2.0 to 19.0 meters respectively. This corresponded to 747 degrees of freedom (DOF) for the fit.

\subsubsection{Monte Carlo Studies of the Measurement Method}

Monte Carlo studies provided information about the necessity of constraining the normalization and the validity of the method used in measuring $\eta_{+-0}$ The need to independently measure the normalization of the number of kaons produced at the target can be demonstrated by the following Monte Carlo study of the measurement of $\eta_{+-0}$ 
A Monte Carlo with $\eta_{+-0}$ equal to zero was used to generate a "data" sample with statistics of 30,000 events. This sample was then analyzed in the same way as the $K_{s}$ lifetime (Section 9.4 .1 ) by fitting $\eta_{+-0}$ and the normalization simultaneously and yielded the result:

$$
\begin{array}{rlrl}
\operatorname{Re}\left\{\eta_{+-0}\right\} & =0.70 \pm 0.05 & \chi^{2} / \mathrm{DOF} & =1.06 \\
\operatorname{Im}\left(\eta_{+-0}\right\} & =-0.94 \pm 0.18 & \text { (fitted) } N_{K}^{M C}(0) & =(3.78 \pm 0.06) \times 10^{10} \\
\text { (Monte Carlo input value) } N_{K}^{M C}(0) & =4.54 \times 10^{10}
\end{array}
$$

The results of the real and imaginary parts of $\eta_{+-0}$ are very far from the input value. The correlation matrix is:

$$
\begin{array}{lcc} 
& \operatorname{Re}\left\{\eta_{+-0}\right\} & \operatorname{Im}\left\{\eta_{+-0}\right\} \\
\operatorname{Im}\left\{\eta_{+-0}\right\} & 0.73 & \\
N_{K}^{M C}(0) & -0.80 & -0.23
\end{array}
$$

Clearly the high correlation between the normalization and $\eta_{+-0}$ distorts the results. The reason for this correlation is that the long "wavelength" of the $\eta_{+-0}$ oscillation superimposed on the exponential as seen in Figure 1.5 can be partially interpreted as simply a shift in the overall normalization.

Since this "data" sample was generated with a Monte Carlo, the actual number of kaons produced at the target was known. It was $20 \%$ larger $\left(4.54 \times 10^{10}\right)$ than that value found by the analysis. If the normalization were independently known to $1 \%$ (i.e. $4.54 \pm 0.05 \times 10^{10}$ ), it can be used as a constraint on the normalization of equation 9.6. Thus the normalization $N_{K}^{D}(0)$ of equation 9.6 is constrained by an overall $\chi^{2}$ term:

$$
\frac{\left(N_{K 2}(0)-N_{K}^{D}(0)\right)^{2}}{\sigma_{N K 2}^{2}}
$$

where $N_{K}^{D}(0)$ is the fitted normalization of equation 9.6 and $N_{K 2}(0)$ is the normalization determined independently either from the Monte Carlo input or, in the case of the data, from the $\mathrm{K} \rightarrow 2 \pi$ sample and $\sigma_{\mathrm{NK} 2}$ is the uncertainty of the independent normalization determination. The resultant measurement of $\eta_{+-0}$ from the Monte Carlo $\left(\eta_{+-0}\right.$ input $\left.=0.0\right)$ was: 


$$
\begin{array}{lll}
\operatorname{Re}\left\{\eta_{+-0}\right\}=0.03 \pm 0.05 & \chi^{2} / \mathrm{DOF}=1.05 \\
\operatorname{Im}\left[\eta_{+-0}\right\}=0.006 \pm 0.03 & N_{K}^{M C}(0)=(4.54 \pm 0.05) \times 10^{10}
\end{array}
$$

Now the experimental agreement with the input value is excellent. The correlation matrix still shows a high correlation between the normalization and $\eta_{+-0}$, but due to the ouside constraint on the normalization, this correlation is now not a problem in the measurement of $\eta_{+-0}$.

All the normalization values determined from the $K_{2 \pi}$ sample are known better than $1 \%$ and thus should sufficiently constrain the normalization in order to determine a realistic measurement of $\eta_{+-0}$.

Having shown that the analysis procedure with an independent normalization constraint does not generate a spurious signal, it was then necessary to test that the technique would detect a signal if one were present. The Monte Carlo simulation program was used to generate a "data" sample with the same statistics as the data with $\operatorname{Re}\left\{\eta_{+-0}\right\}=-0.13$ and $\operatorname{Im}\left\{\eta_{+-0}\right\}=0.14$ in the decay subroutine. Fitting these Monte Carlo events as data in my program measured:

$$
\begin{array}{ll}
\operatorname{Re}\left\{\eta_{+-0}\right\}=-0.17 \pm 0.04 & \chi^{2} / \mathrm{DOF}=1.08 \\
\operatorname{Im}\left\{\eta_{+-0}\right\}=0.14 \pm 0.02 & N_{K}^{M C}(0)=(4.60 \pm 0.01) \times 10^{10}
\end{array}
$$

This "measurement" is within one standard deviation of the input value verifying that the fitting technique could accurately measure $\eta_{+-0}$.

\subsubsection{Biases}

The measurement of $\eta_{+-0}$ is a difficult one since the expected signal is small. A measurement of the necessary accuracy requires near perfect understanding of the acceptance of the experiment. This experiment was designed such that the upstream data, if necessary, could be used to help measure that acceptance without relying on a perfect simulation of the acceptance.

The upstream $\mathrm{K} \rightarrow 3 \pi$ data sample should have a negligible signal for $\eta_{+-0}$ since the $\eta_{+-0}$ term is short lived. To check that this was the case, the upstream data were analyzed as if it were data from the downstream target. The number of data events used was 19,500 . The signal measured from the upstream west data was, (upstream 
east data results are given in parentheses):

$$
\begin{aligned}
\operatorname{Re}\left\{\eta_{+-0}\right\}=-0.030 \pm 0.062(-0.240 \pm 0.063) \quad \chi^{2} / \mathrm{DOF}=1.24(1.16) \\
\operatorname{Im}\left\{\eta_{+-0}\right\}=0.149 \pm 0.036(0.079 \pm 0.030) N_{K}^{D}(0)=(2.72 \pm 0.03) \times 10^{10} \\
(\text { east }) N_{K}^{D}(0)=(2.14 \pm 0.03) \times 10^{10}
\end{aligned}
$$

This points to a bias in the data. Some part of the apparatus acceptance was not simulated by the Monte Carlo and exhibits itself as a "fake" $\eta_{+-0}$ signal. Thus the Monte Carlo acceptance, $\mathrm{A}^{\mathrm{MC}}$, is not identical to the data and equation 9.5 is not a completely valid theoretical model. It is also possible that the bias could be different for the east and west beams. The real part of $\eta_{+-0}$ varies by $\sim 3$ standard deviations between the east and west beams and the imaginary part varies by two standard deviations.

The downstream results are not completely consistent with the upstream bias signals. Using this technique on the DSW data found (DSE data results are given in parentheses):

$$
\begin{aligned}
\operatorname{Re}\left\{\eta_{+-0}\right\}=-0.329 \pm 0.034(-0.250 \pm 0.043) \quad \chi^{2} / \mathrm{DOF}=1.08(1.12) \\
\operatorname{Im}\left\{\eta_{+-0}\right\}=0.120 \pm 0.016(0.160 \pm 0.020) N_{K}^{D}(0)=(4.93 \pm 0.01) \times 10^{10} \\
(\text { east }) N_{K}^{D}(0)=(5.29 \pm 0.04) \times 10^{10}
\end{aligned}
$$

The downstream west (DSW) $\operatorname{Re}\left[\eta_{+-0}\right\}$ signal is 5 standard deviations below the upstream west bias signal and the downstream east (DSE) $\operatorname{Im}\left[\eta_{+-0}\right]$ signal is 2.5 standard deviations above the upstream east bias signal. The two downstream signals are quite close, varying by 2 standard deviations or less in both the real and imaginary parts of $\eta_{+-\mathbf{0}}$.

In Figure 9.2 the $z$ vertex distribution was shown for the downstream east data sample. To see if the measured bias signal was indeed due to the slight excess of upstream decays apparent in all target samples, a generated Monte Carlo "data" sample with $\eta_{+-0}$ approximately equal to the measured bias signal $\left(\operatorname{Re}\left\{\eta_{+-0}\right\}=-0.23\right.$ and $\left.\operatorname{Im}\left\{\eta_{+-0}\right\}=0.16\right)$ for the east beam was compared to the downstream east data sample. The difference between the Monte Carlo and data distributions are shown in Figure 9.3 for the bias signal Monte Carlo and a zero signal Monte Carlo. 


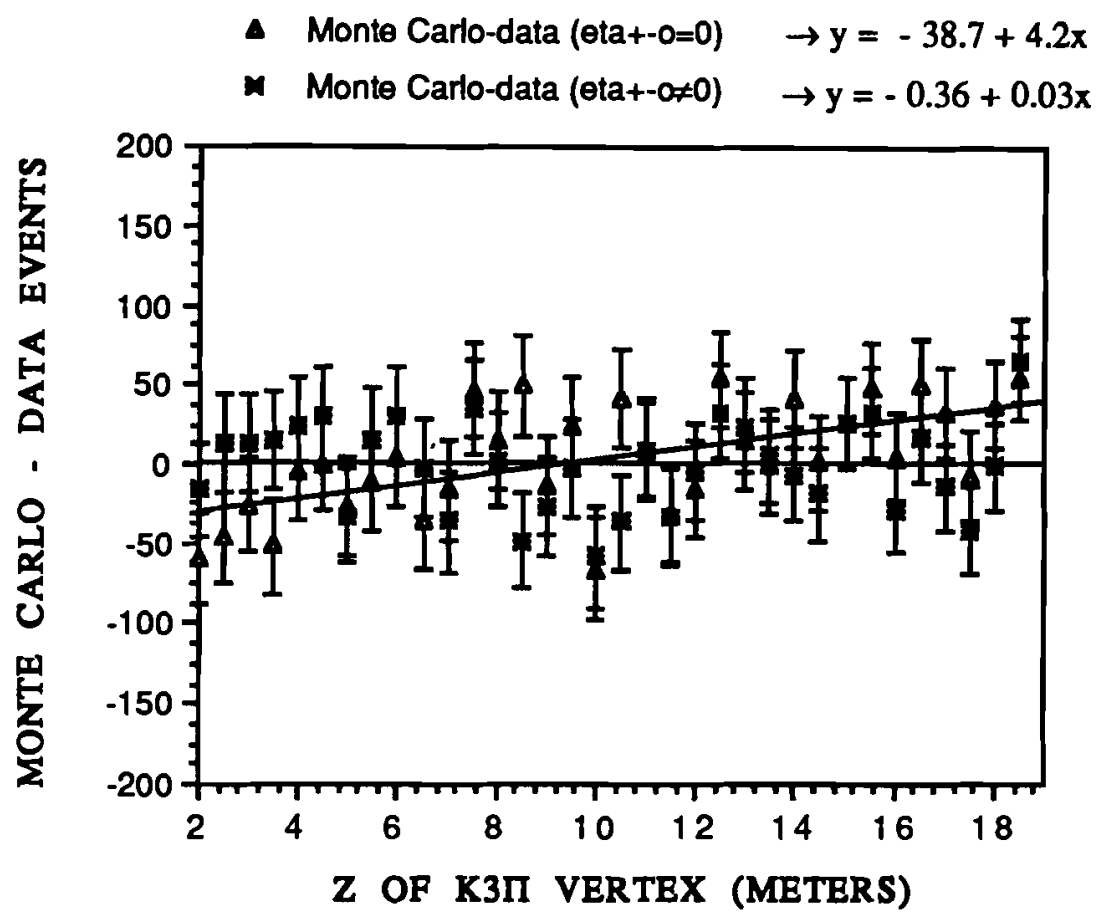

Figure 9.3 $\mathrm{K}_{3 \pi}$ Z-Vertex Monte Carlo Minus Data

Straight line fits are shown for the two Monte Carlo minus data samples in Figure 9.3. The agreement between the bias signal Monte Carlo and the data is quite good. This shows that a real signal would exhibit itself as the $z$ distribution that we observe.

At this juncture two different approaches to correcting for this bias are possible. One uses maximum likelihood and the Monte Carlo acceptance and calculates:

$$
\eta_{+-o}^{\prime}=\eta_{+-o}^{b}+\eta_{+-o}
$$

where $\eta_{+-o}^{b}$ is the signal associated with the bias and $\eta_{+-o}$ is the real signal. The other method uses the $\chi^{2}$ method and calculates a correction factor to the Monte Carlo acceptance using the upstream target data. Both methods must characterize both the upstream and the downstream data simultaneously in order to correct for the bias. The following expressions are derived to measure $\eta_{+-0}$ for a single beam, either east 
or west. To measure $\eta_{+-0}$ for both beams, a second expression, identicle to the the first but corresponding to the other beam is added.

\subsubsection{Maximum Likelihood and $\eta^{b_{+-0}}$}

In this method, for a single beam, there are two terms to the maximum likelihood function; one corresponding to the downstream data and one corresponding to the upstream data. The expected downstream term is:

$$
\begin{gathered}
N_{3 \pi}^{E_{d s}}\left(p_{i}, z_{j}, \mathrm{~d} s\right)=A_{3 \pi}^{M C}\left(p_{i}, z_{j}, \mathrm{~d} s\right) n_{3 \pi}\left(\eta_{+-o}^{b}+\eta_{+-o}, p_{c i}, z_{j}, \mathrm{~d} s\right) * \\
* N_{K}^{D}(0, \mathrm{~d} s) B R_{3 \pi}
\end{gathered}
$$

where $\mathrm{ds}$ refers to the downstream target. $A_{3 \pi}^{M C}\left(p_{i}, z_{j}, \mathrm{~d} s\right)$ is the acceptance determined by the Monte Carlo for the downstream target, $N_{K}^{D}(0, \mathrm{~d} s)$ is the number of kaons produced from the downstream target, which is constrained by the value determined from the $K \rightarrow 2 \pi$ data sample as per equation 9.14, and $n_{3 \pi}\left(\eta_{+-o}^{b}+\eta_{+-o}, p_{c i}, z_{j}, \mathrm{~d} s\right)$ is the decay equation, defined in equation 9.1 , with respect to the downstream target. $\eta_{+-o}^{b}$ is the signal introduced by the bias in the data which is measured by the upstream data term:

$$
\begin{gathered}
N_{3 \pi}^{E_{u s}}\left(p_{i}, z_{j}, u s\right)=A_{3 \pi}^{M C}\left(p_{i}, z_{j}, u s\right) n_{3 \pi}\left(\eta_{+-o}^{b}, p_{c i}, z_{j}, d s\right) * \\
* N_{K}^{D}(0, u s) B R_{3 \pi}
\end{gathered}
$$

where the decay equation, $n_{3 \pi}\left(\eta_{+-o}^{b}, p_{c i}, z_{j}, d s\right)$, is with respect to the downstream target and thereby measures the bias signal, $\eta_{+-o}^{b}$, common to both samples. For one beam, i.e. east or west, the maximum likelihood equation is:

$$
\begin{aligned}
& \mathbf{C}\left(p_{i}, z_{j}\right)=N_{3 \pi}^{E_{u s}}\left(p_{i}, z_{j}, u s\right)-N_{3 \pi}^{D}\left(p_{i}, z_{j}, u s\right) \\
&-N_{3 \pi}^{D}\left(p_{i}, z_{j}, u s\right) \ln \left(\frac{N_{3 \pi}^{E_{u s}}\left(p_{i}, z_{j}, u s\right)}{N_{3 \pi}^{D}\left(p_{i}, z_{j}, u s\right)}\right) \\
&+N_{3 \pi}^{E_{d s}}\left(p_{i}, z_{j}, d s\right)-N_{3 \pi}^{D}\left(p_{i}, z_{j}, d s\right) \\
&-N_{3 \pi}^{D}\left(p_{i}, z_{j}, d s\right) \ln \left(\frac{N_{3 \pi}^{E_{d s}}\left(p_{i}, z_{j}, d s\right)}{N_{3 \pi}^{D}\left(p_{i}, z_{j}, d s\right)}\right)
\end{aligned}
$$


to which one adds the two overall normalization constraints from $\mathrm{K} \rightarrow 2 \pi$ decays:

$$
\frac{\left(N_{K}^{D}(0, d s)-N_{K_{2 \pi}}^{D}(0, d s)\right)^{2}}{\sigma\left(N_{K_{2 \pi}}^{D}(0, d s)\right)^{2}}+\frac{\left(N_{K}^{D}(0, u s)-N_{K_{2 \pi}}^{D}(0, u s)\right)^{2}}{\sigma\left(N_{K_{2 \pi}}^{D}(0, u s)\right)^{2}}
$$

Here $N_{K}^{D}(0, u s)$ and $N_{K}^{D}(0, d s)$ are the upstream and downstream $\mathrm{K} \rightarrow 3 \pi$ normalization constants which are constrained by $N_{K_{2 \pi}}^{D}(0, u s)$ and $N_{K_{2 \pi}}^{D}(0, d s)$ determined from $\mathrm{K} \rightarrow 2 \pi$ decays (shown in Table 9.2), with $\sigma\left(N_{K_{2 \pi}}^{D}(0, u s)\right)$ and $\sigma\left(N_{K_{2 \pi}}^{D}(0, d s)\right)$ being their errors. The results are summarized in Table 9.4 for each beam seperately.

All theories predict the major contribution to CP symmetry violation in kaons is from the mass matrix mixing and thus independent of the decay. The decay contribution to CP symmetry violation is largely imaginary and thus one can use the constraint that $\operatorname{Re}\left\{\eta_{+-0}\right\} \approx \operatorname{Re}\left\{\eta_{+-}\right\} \approx 0.0016 \approx 0.0$ within the errors of this experiment. Results are shown in Table 9.4 for $\operatorname{Re}\left\{\eta_{+-0}\right\}$ constrained to zero. For the east beam the results are relatively consistent with zero and both the bias signal and $\eta_{+-0}$ vary little when the real part of $\eta_{+-0}$ is constrained to zero. For the west beam the real part of $\eta_{+-0}$ is 4.5 standard deviations from zero which, I believe, is due to an inaccurate measurement of the normalization for the upstream west target/downstream east target combination due to some apparatus or beam irregularities not included in the Monte Carlo. When the real part of $\eta_{+-0}$ is constrained to zero, the normalization absorbs much of the $\eta_{+-0}$ signal. However, the imaginary part of $\eta_{+-0}$ and the real part of $\eta_{+-0}$ are $70 \%$ correlated (Section 9.5.1), so that some of the 4.5 sigma signal is absorbed by the imaginary part of $\eta_{+-0}$. This leads to a six standard deviation difference between the east and west beam for the imaginary part of $\eta_{+-0}$ when the real part of $\eta_{+-0}$ is constrained to zero. An overall correction to the upstream west and downstream east normalization (isochronous data conditions) of $6 \%$ yeilds good agreement between the east and west beam results. These results are also consistent with zero (within 1/2 a standard deviation) for the imaginary part of $\eta_{+-0}$ both with and without the real part of $\eta_{+-0}$ constrained. The 
real part of $\eta_{+-0}$ remains a three standard deviation effect. Thus the possibility of an overall efficiency which is not included correctly in the final analysis is most likely. Fortunately when both beams are fit in the end this type of systematic error will tend to cancel.

To verify this correlation effect using this method of measuring $\eta_{+-0}$, both $\eta_{+-0}$ and $\eta^{\mathrm{b}}{ }_{+-0}$ were measured using a Monte Carlo simulation program to generate a "data" sample with $\operatorname{Re}\left\{\eta_{+-0}\right\}=-0.23$ and $\operatorname{Im}\left\{\eta_{+-0}\right\}=0.16$ and $\eta^{b}{ }_{+-0}$ equal to zero. The normalization of kaons produced on the target was constrained to that of the Monte Carlo simulation. The results are given in the third row of Table 9.4. The measured signal is within one standard deviation of the input signal for both $\eta_{+-0}$ and $\eta^{b}+-0$. When the real part of $\eta_{+-0}$ is constrained to zero, one can see how the real part of the bias signal absorbs the signal from the real part of $\eta_{+-0}$.

\begin{tabular}{|c|c|c|c|c|c|}
\hline \multirow{2}{*}{ BEAM } & & \multicolumn{4}{|c|}{ Maximum Likelihood Results of $\eta_{+-o}^{b}$ and $\eta_{+\infty}$} \\
\hline & & $\operatorname{Re}\left\{\eta_{+\infty}\right\}$ & $\operatorname{Im}\left[\eta_{+-0}\right]$ & Normalization & $\overline{x_{\text {DOF }}^{2}}$ \\
\hline \multirow{4}{*}{$\begin{array}{l}\text { WEST } \\
\text { BEAM }\end{array}$} & $\eta_{+-0}$ & $-0.299 \pm 0.071$ & $-0.029 \pm 0.040$ & $4.93 \pm 0.01 \times 10^{10}$ & \multirow{4}{*}{$\begin{array}{l}1.32 \\
--1.35\end{array}$} \\
\hline & $\eta_{+-0}^{b}$ & $-0.030 \pm 0.063$ & $0.149 \pm 0.036$ & $2.72 \pm 0.03 \times 10^{10}$ & \\
\hline & $\eta_{+-0}^{-}$ & $0 . \overline{0}-$ & $-0 . \overline{11} \pm 0.029$ & $4.90 \pm 0.01 \times 10^{10}$ & \\
\hline & $\eta_{+-0}^{b}$ & $-0.258 \pm 0.029$ & $0.210 \pm 0.025$ & $2.71 \pm 0.03 \times 10^{10}$ & \\
\hline \multirow{4}{*}{$\begin{array}{l}\text { EAST } \\
\text { BEAM }\end{array}$} & $\eta_{t-0}$ & $-0.010 \pm 0.076$ & $0.081 \pm 0.036$ & $5.29 \pm 0.04 \times 10^{10}$ & \multirow{4}{*}{$\begin{array}{c}1.24 \\
--\frac{1}{1.24}\end{array}$} \\
\hline & $\eta_{+-0}^{b}$ & $-0.240 \pm 0.063$ & $0.079 \pm 0.030$ & $2.14 \pm 0.02 \times 10^{10}$ & \\
\hline & $\bar{\eta}_{+-0}$ & $0 . \overline{0}--$ & $0 . \overline{07} 9 \pm 0 . \overline{034}$ & $5.29 \pm 0.04 \times 10^{10}$ & \\
\hline & $\eta_{+-0}^{b}$ & $-0.248 \pm 0.036$ & $0.080 \pm 0.029$ & $2.14 \pm 0.02 \times 10^{10}$ & \\
\hline \multirow{4}{*}{$\begin{array}{l}\text { SIGNAL } \\
\text { MONTE } \\
\text { CARLO }\end{array}$} & $\eta_{t-0}$ & $-0.151 \pm 0.072$ & $0.133 \pm 0.051$ & $5.15 \pm 0.01 \times 10^{10}$ & \multirow{2}{*}{$\begin{array}{l}1.27 \\
--\end{array}$} \\
\hline & $\eta_{+-0}^{b}$ & $-0.031 \pm 0.062$ & $0.023 \pm 0.021$ & $2.97 \pm 0.03 \times 10^{10}$ & \\
\hline & $\eta_{t-0}$ & -0.0 & $0 . \overline{07} 1 \pm 0 . \overline{0} 32$ & $5.15 \pm 0.01 \times 10^{10}$ & 128 \\
\hline & $\eta_{+-0}^{b}$ & $-0.152 \pm 0.031$ & $0.061 \pm 0.032$ & $3.00 \pm 0.03 \times 10^{10}$ & \\
\hline
\end{tabular}

Table 9.4 Maximum Likellhood Results of $\eta+-0$ 


\subsection{4 $\chi^{2}$ Acceptance Method}

The bias in the data exists because the Monte Carlo is not correctly calculating the spectrometer and reconstruction acceptance term $A_{3 \pi}^{D}\left(p_{i}, z_{j}\right)$ of equation 9.4. In this method a correction to the acceptance is determined using the upstream data. From equation 9.4, for the upstream data:

$$
\begin{aligned}
N_{3 \pi}^{D}\left(p_{i}, z_{j}, u s\right) & =A_{3 \pi}^{M C}\left(p_{i}, z_{j}, u s\right) A_{3 \pi}^{c f}\left(p_{i}, z_{j}\right) n_{3 \pi}\left(\eta_{+-o}, p_{c i}, z_{j}, u s\right) \\
& * N_{K}^{D}(0, u s) B R_{3 \pi}
\end{aligned}
$$

where $A_{3 \pi}^{c f}\left(p_{i}, z_{j}\right)$ is the correction factor used to obtain the actual acceptance from $A_{3 \pi}^{M C}\left(p_{i}, 2_{j}\right)$ where us corresponds to values taken with respect to the upstream target and $\eta_{+-0}$ is the real CP symmetry violating signal. Solving for the correction factor:

$$
A_{3 \pi}^{c f}\left(p_{i}, z_{j}\right)=\frac{N_{3 \pi}^{D}\left(p_{i}, z_{j}, u s\right)}{A_{3 \pi}^{M C}\left(p_{i}, z_{j}, u s\right) n_{3 \pi}\left(\eta_{+-o}, p_{c i}, z_{j}, u s\right) N_{K}^{D}(0, u s) B R_{3 \pi}}
$$

Here $N_{3 \pi}^{D}\left(p_{i}, z_{j}, u s\right)$ is the upstream data in $\mathrm{p}$ and $\mathrm{z}$ bins. For the downstream target data the expected value is:

$$
\begin{gathered}
N_{3 \pi}^{E_{d s}}\left(p_{i}, z_{j}, d s\right)=A_{3 \pi}^{M C}\left(p_{i}, z_{j}, d s\right) A_{3 \pi}^{c f}\left(p_{i}, z_{j}\right) n_{3 \pi}\left(\eta_{+-o}, p_{c i}, z_{j}, d s\right) * \\
* N_{K}^{D}(0, d s) B R_{3 \pi}
\end{gathered}
$$

which becomes, assuming the upstream and downstream target correction factors are the same:

$$
\begin{aligned}
N_{3 \pi}^{E_{d s}}\left(p_{i}, z_{j}, d s\right) & = \\
& \frac{N_{K}^{D}(0, d s) A_{3 \pi}^{M C}\left(p_{i}, z_{j}, d s\right) n_{3 \pi}\left(\eta_{+-o}, p_{c i}, z_{j}, d s\right)}{N_{K}^{D}(0, u s) A_{3 \pi}^{M C}\left(p_{i}, z_{j}, u s\right) n_{3 \pi}\left(\eta_{+-o}, p_{c i}, z_{j}, u s\right)} N_{3 \pi}^{D}\left(p_{i}, z_{j}, u s\right)
\end{aligned}
$$

$\eta_{+-0}$ is then measured using the expected value from equation 9.26 and the $\chi^{2}$ function in equation 9.9 along with an overall normalization constraint: 


$$
\frac{\left(\frac{N_{K 2}^{D}(0, d s)}{N_{K 2}^{D}(0, u s)}-X X(1)\right)^{2}}{\sigma_{N K 2}^{2}}
$$

with:

$$
X X(1)=\frac{N_{K}^{D}(0, d s)}{N_{K}^{D}(0, u s)}
$$

$\sigma_{N K 2}^{2}$ is the uncertainty in the ratio of the $\mathrm{K} \rightarrow 2 \pi$ normalizations, $\frac{N_{K 2}^{D}(0, d s)}{N_{K 2}^{D}(0, u s)}$. Constraining the normalization as a ratio of the downstream and upstream normalizations is an advantage of the $\chi^{2}$ method. When the normalization is constrained as a ratio, common factors in the numerator and denominator cancel diminishing possible sources of systematic uncertainties. The results of the $\chi^{2}$ fit for both beams are given in Table 9.5. The results are within 1.5 standard deviation of those measured by the maximum likelihood method (Table 9.4). As in the maximum likelihood method the real part of $\eta_{+-0}$ for the west beam is inconsistent with zero. When the real part of $\eta_{+-o}$ is constrained to zero the imaginary portion for the west beam becomes a five sigma signal. The east beam results have a three sigma signal for the imaginary part of $\eta_{+-0}$ and are stable when the real part of $\eta_{+-0}$ is constrained to zero.

The last row in Table 9.5 gives the result obtained from a Monte Carlo program which generated "data" with $\operatorname{Re}\left\{\eta_{+-0}\right\}=-0.23$ and $\operatorname{Im}\left\{\eta_{+}\right\}=0.16$ and no bias signal. The normalization of kaons was constrained to the normalization value from that same Monte Carlo. The resultant measurement of $\eta_{+-0}$ is very good when both the real and imaginary part of $\eta_{+-0}$ are unconstrained. When the real part of $\eta_{+-0}$ is constrained to zero, the imaginary part of $\eta_{+-0}$ changes by one standard deviation due to the $70 \%$ correlation between the real and imaginary part of $\eta_{+-0}$.

An advantage to this method is that the normalization of the kaons is constrained as a ratio of the number produced at the downstream target to the number produced at the upstream target. This number is constant for each beam (east and west) regardless of the data sample selection. This allows one to remove $p$ and $z$ bins from 
the $\mathrm{K} \rightarrow 3 \pi$ data sample to check the stability of the measurement of $\eta_{+-0}$ without recalculating the normalization. Also the acceptance comes into the $\chi^{2}$ method as a ratio of the downstream to upstream acceptance. This helps decrease systematic uncertainties as common acceptance factors will cancel between the upstream and downstream samples, making overall efficiency values less important versus the maximum likelihood method where no such cancellation occurs and thus overall corrections for efficiencies must be calculated and included in the final formulation. An advantage to the maximum likelihood method is that the bias signal can be measured for each beam independently or constrained to be the same for both beams.

\begin{tabular}{|c|c|c|c|c|}
\hline \multirow{2}{*}{ BEAM } & \multicolumn{4}{|c|}{ Chi-Squared Minimization Results of } \\
\hline & $\operatorname{Re}\left[\eta_{+-\infty}\right]$ & $\operatorname{Im}\left[\eta_{+-\infty}\right]$ & Normalization & $x_{\text {/DOF }}^{2}$ \\
\hline WEST BEAM & $\begin{array}{c}-0.319 \pm 0.046 \\
----- \\
0.0\end{array}$ & $\begin{array}{r}-0.080 \pm 0.026 \\
--0.143 \pm 0.027\end{array}$ & $\begin{array}{c}1.82 \pm 0.02 \\
------ \\
1.78 \pm 0.02\end{array}$ & $\begin{array}{r}0.95 \\
-- \\
0.95\end{array}$ \\
\hline EAST BEAM & $\begin{array}{c}-0.096 \pm 0.067 \\
----- \\
0.0\end{array}$ & $\begin{array}{r}0.135 \pm 0.076 \\
-\overline{0.118 \pm 0.026}\end{array}$ & $\begin{array}{c}2.48 \pm 0.04 \\
-\overline{2.46 \pm 0.03}--\end{array}$ & $\begin{array}{r}0.96 \\
-- \\
0.96\end{array}$ \\
\hline $\begin{array}{l}\text { SIGNAL } \\
\text { MONTE } \\
\text { CARLO }\end{array}$ & $\begin{array}{c}-0.231 \pm 0.072 \\
---- \\
0.0\end{array}$ & $\begin{array}{c}0.142 \pm 0.031 \\
----- \\
0.100 \pm 0.031\end{array}$ & $\begin{array}{l}1.74 \pm 0.02 \\
----- \\
1.72 \pm 0.02\end{array}$ & $\begin{array}{r}0.75 \\
-- \\
0.76\end{array}$ \\
\hline
\end{tabular}

Table $9.5 \chi^{2}$ Minimumization Results of $\eta_{+-0}$

\subsubsection{Dual Beam Results}

There is a great advantage to combining all four beams for a measurement. Data for single beam results, while having similar acceptance due to phase space, has the disadvantage of being taken at different times for the upstream and downstream target samples. Even in the $\chi^{2}$ method where overall efficiencies cancel in as much as they are consistent between upstream and downstream samples, the cancellation is 
not perfect due to this. But in the final formulation if one uses the $\chi^{2}$ method with all four targets, this "systematic" effect is greatly reduced. Thus the differences between the individual east and west beam results are not an accurate measurement of an overall systematic uncertainty but rather an indication of the variances of efficiences with time not fully included in the Monte Carlo. Combining all four beams, the result for $\eta_{+-0}$ is shown in Table 9.7. The number of events that were used in determining this result are given in Table 9.6.

\begin{tabular}{|c|c|}
\hline TARGET & Number of Events \\
\hline downstream west & $43 \mathbf{k}$ \\
\hline upstream west & $21 \mathbf{k}$ \\
\hline downstream east & $39 \mathbf{k}$ \\
\hline upstream east & $19 \mathbf{k}$ \\
\hline
\end{tabular}

Table 9.6 Number of Events In Final Sample

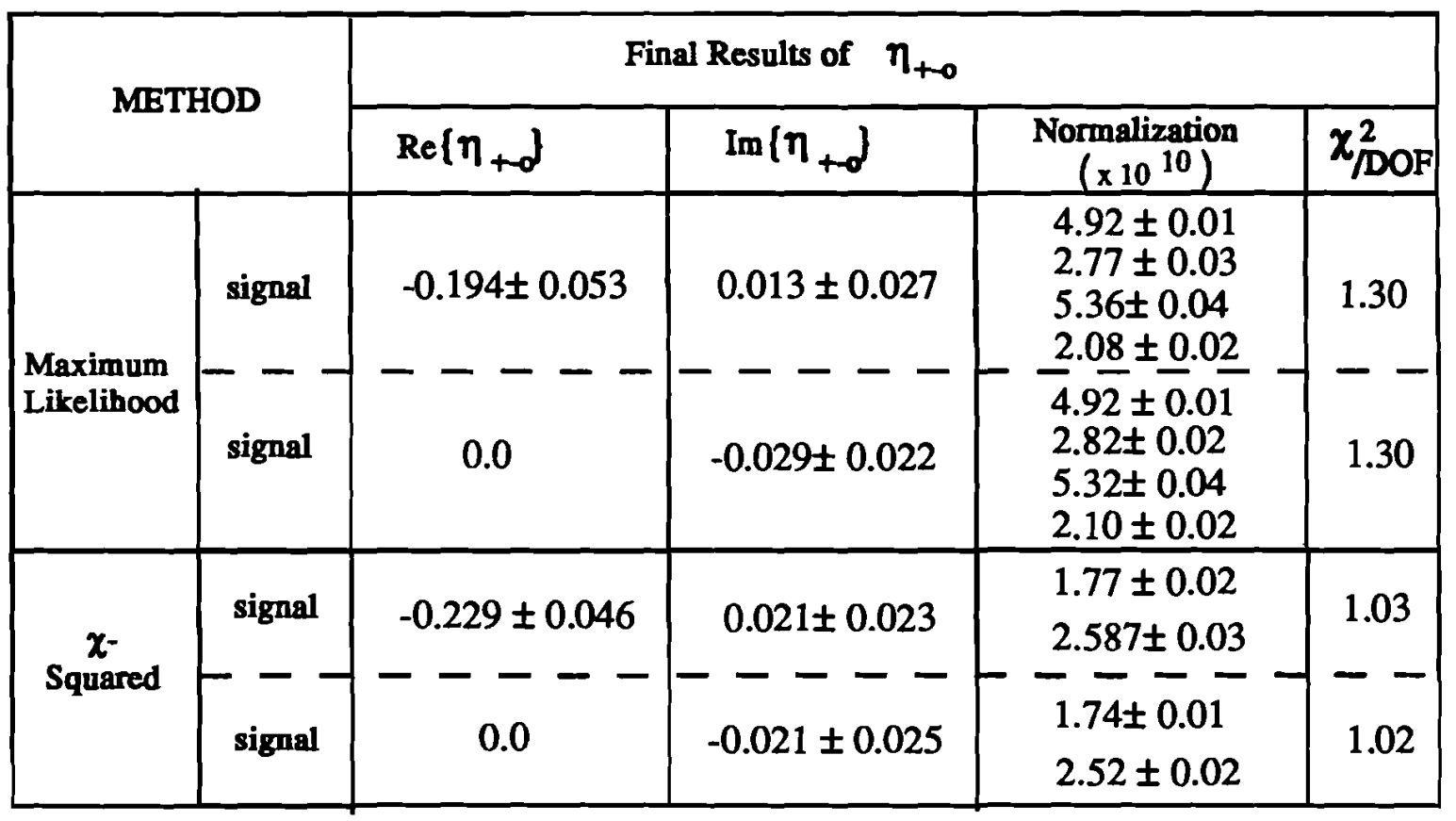

Table 9.7 Final Result of the Measurement of $\eta_{+-0}$ 
The $\chi^{2}$ and maximum likelihood results agree within $\sim 1 / 2$ standard deviation. The results for both the $\chi^{2}$ and the maximum likelihood method for the $\operatorname{Re}\left\{\eta_{+-0}\right\}$ are $\sim 4$ standard deviations (statistically) from zero. On the other hand, the important meassurement of $\operatorname{Im}\left\{\eta_{+-0}\right\}$ is consistent with zero regardless of whether $\operatorname{Re}\left\{\eta_{+-0}\right\}$ is constrained to zero or not.

The correlation matrices for the two results above with $\operatorname{Re}\left\{\eta_{+-0}\right\}$ unconstrained are shown in Tables 9.8a and b.

\begin{tabular}{|c|c|c|c|c|c|c|c|}
\hline $\begin{array}{l}\text { Maximum } \\
\text { Likelihood } \\
\text { Method }\end{array}$ & 荇 & 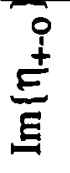 & $z^{\frac{3}{8}}$ & $z^{3}$ & $\frac{z^{+}}{\mathscr{q}}$ & 具 & $z^{8}$ \\
\hline $\operatorname{Im}\left\{\eta_{+-0}\right\}$ & -.52 & & & & & & \\
\hline $\mathrm{N}_{\mathrm{dw}}$ & -.12 & -.08 & & & & & \\
\hline $\mathbf{N}_{\mathbf{u w}}$ & .46 & 12 & 0.0 & & & & \\
\hline $\operatorname{Re}\left\{\eta_{+-0}^{b}\right\}$ & -.87 & .48 & 0.0 & -.53 & & & \\
\hline $\operatorname{Im}\left[\eta_{+0}^{b}\right\}$ & .47 & -.88 & 0.0 & -13 & -.54 & & \\
\hline$N_{d e}$ & -.18 & -.13 & .23 & 0.0 & 0.0 & 0.0 & \\
\hline $\mathrm{N}_{\text {we }}$ & .46 & .12 & 0.0 & .53 & -.13 & -.13 & 0.0 \\
\hline
\end{tabular}

Table 9.8a Correlation Matrices in the Measurement of $\eta_{+-0}$ (Maximum Llkellhood method)

The correlation matrix for the $\chi^{2}$ method shows all correlations are $50 \%$ or less. The same is true for the maximum likelihood method, except for the correlations between $\eta^{b}{ }_{+-0}$ and $\eta_{+-0}$. But this is not a problem since $\eta^{b}{ }_{+-0}$ is constrained by the upstream data samples. 


\begin{tabular}{|c|c|c|c|}
\hline $\begin{array}{c}\chi^{2} \\
\text { Method }\end{array}$ & $\frac{E^{+}}{\tilde{Q}}$ & & $\left.z^{z}\right|_{z}$ \\
\hline $\operatorname{Im}\left\{\eta_{+-0}\right.$ & .03 & & \\
\hline$\frac{\mathrm{N}_{\mathrm{dw}}}{\mathrm{N}_{\mathrm{uw}}}$ & -.45 & -.42 & \\
\hline$\frac{N_{d e}}{N_{u e}}$ & -.42 & -.49 & .38 \\
\hline
\end{tabular}

Figure 9.8b Correlation Matrices in the Measurement of $\eta_{+-0}\left(\alpha^{2}\right.$ method)

Using the maximum likelihood method, one can investigate the measurement of $\eta_{+-0}$ assuming $\eta_{\text {east }}^{b}=\eta_{\text {west }}^{b}$. The result in Table 9.7 allows for different bias signals between the east and west beams using the maximum likelihood method. The bias signals were,

$$
\begin{aligned}
& \text { (west) } \operatorname{Re}\left[\eta^{\mathrm{b}}{ }_{+-0}\right\}=-0.107 \pm 0.054 \\
& \text { (east) } \operatorname{Re}\left[\eta^{\mathrm{b}}{ }_{+-0}\right\}=-0.130 \pm 0.048
\end{aligned}
$$$$
\operatorname{Im}\left[\eta_{+-0}^{b}\right\}=0.116 \pm 0.027
$$$$
\operatorname{Im}\left\{\eta^{\mathrm{b}}{ }_{+-0}\right\}=0.126 \pm 0.025
$$

For $\eta_{\text {east }}^{\mathrm{b}}=\eta_{\text {west }}^{\mathrm{b}}$ the result for $\eta_{+-0}$ varied by less than half a standard deviation from the results shown in Table 9.7. The correlation matrix for the maximum likehood method shown in Table 9.8 is for $\eta^{b}$ east $=\eta^{b}$ west .

A value of $D_{K K}=0.5$ (see equation 9.1) was used in determining all the results (see Appendix D)

\subsubsection{Overall Systematic Uncertainties}

The statistical uncertainties of a measurement can be misleading if large systematic uncertainties exist. The difference between the measurements of $\eta_{+-0}$ for the east and west beams hints at a possible overall systematic uncertainty. More subtle sources of systematic uncertainties can be introduced by the method of data selection. The data selection criteria that remove the most events are the most suspect for causing systematic uncertainties. In the final data selection criteria, described in Section 8.3.2, the beam phase space requirements and the momentum and decay vertex limits are the most severe and their effects on the measurement 
were examined in detail. I also examined the effects of the normalization on the measurement of $\eta_{+-0}$.

The strict requirements on $M_{\theta+\theta_{-}}, M_{\Lambda}$ and $P_{\pi+} / P_{\pi-}$ of Section 8.4.2 removed $35 \%$ of the data sample while not changing $\eta^{b}{ }_{+-0}, \eta_{+-0}$ or improving any comparison plots between Monte Carlo and data. This helped show that these tight requirements were not necessary, and that the result was stable under this large variation in the data sample.The only other set of requirements that were significant were the neutral beam phase space requirements of Table 8.2 which removed $\sim 10 \%$ of the data from each beam. Tightening these by $10 \%$ and remeasuring $\eta_{+-o}$ provided another measure of the systematic uncertainty. The results are shown in Table 9.9. The results all are consistent with a systematic uncertainty of 0.02 for the real part of $\eta_{+-0}$ and $\sim 0.001$ for the imaginary part of $\eta_{+-0}$. When the real part of $\eta_{+-0}$ is constrained to zero the systematic uncertainty in the imaginary part is still negligible.

\begin{tabular}{|c|c|c|c|c|c|c|}
\hline & \multicolumn{4}{|c|}{ Systematic Errors in the Measurement of } & \multirow{2}{*}{\multicolumn{2}{|c|}{$\frac{\eta_{+-0}}{\substack{\text { Beam cuts } 10 \% \\
\text { tighter }}}$}} \\
\hline & \multicolumn{2}{|c|}{$\begin{array}{l}\text { Origlnal } \\
\text { Cuts }\end{array}$} & \multicolumn{2}{|c|}{$\begin{array}{l}\text { Me+o-cut @ 0.14Gov } \\
\text { 10sigma lambda mass } \\
\text { cut } \\
\text { Slope Ratlo cut .4->2.5 }\end{array}$} & & \\
\hline & $\operatorname{Re}(\eta+\infty)$ & $\operatorname{Im}\left\{\eta_{+-\infty}\right\}$ & $\operatorname{Re}\left\{\eta_{+\infty}\right\}$ & $\operatorname{Im}\left[\eta_{+\infty}\right]$ & $\operatorname{Re}\left[\eta_{+-\infty}\right]$ & $\operatorname{Im}\left\{\eta_{+-0}\right\}$ \\
\hline $\begin{array}{l}\text { signal } \\
--\end{array}$ & $\begin{array}{r}-0.229 \pm \\
-0.046\end{array}$ & $\begin{array}{r}0.021 \pm \\
0.023 \\
-\quad-\quad-\end{array}$ & $\begin{array}{r}-0.250 \pm \\
-0.056\end{array}$ & $\begin{array}{r}0.020 \pm \\
0.030 \\
-\quad-\quad-\end{array}$ & $\begin{array}{r}-0.248 \pm \\
-0.047\end{array}$ & $\begin{array}{r}0.020 \pm \\
0.024 \\
-\quad-~\end{array}$ \\
\hline signal & 0.0 & $\begin{array}{c}-0.021 \pm \\
0.025\end{array}$ & 0.0 & $\begin{array}{r}-0.020 \pm \\
0.028\end{array}$ & & $\begin{array}{r}-0.020 \pm \\
0.026\end{array}$ \\
\hline
\end{tabular}

Table 9.9 Systematlc Uncertainties of $\eta_{+-0}$

Changes in $p$ (momentum of the kaon) and $z$ (decay vertex of the kaon) limits changed the resultant measurements of $\eta_{+-0}$ by at most $1 / 2$ a standard deviation for both the east and west beams. The lower limit on the momentum was varied from $120 \mathrm{Gev}$ to $150 \mathrm{Gev}$, while the upper limit varied from $330 \mathrm{GeV}$ to $370 \mathrm{GeV}$. Below $120 \mathrm{GeV}$, the discrepancy between the Monte Carlo and the data momentum spectrums affected the result by two standard deviations since this discrepancy in the mometum 
spectrums greatly effects the normalization and thus the measurement of $\eta_{+-0} . Z$ of the decay vertex was varied from $1 \mathrm{~m}$ to $4 \mathrm{~m}$ and $16 \mathrm{~m}$ to $19 \mathrm{~m}$ on the lower and upper bounds with the result being that $\eta_{+-o}$ varied by less than one half of a standard deviation. Even the systematic uncertainty determined by varying the $K_{2 \pi}$ normalization values by one standard deviation changed the final result by less than $1 / 2$ a standard deviation both for the $R e\left\{\eta_{+-0}\right\}$ constrained to zero and unconstrained. As far as normalization constraints, the final measurement was least sensitive to the downstream normalizations values. There changes of up to 3 standard deviations varied the result by only $1 / 2$ a standard deviation.

The small variation of the measurement with data selection criteria shows the stability of the final result. Thus the final result, including estimated systematic uncertainties, was,

$$
\begin{aligned}
& \operatorname{Re}\left\{\eta_{+-0}\right\}=-0.229 \pm 0.046 \text { (stat) } \pm 0.023 \text { (syst) } \\
& \operatorname{Im}\left\{\eta_{+-0}\right\}=0.021 \pm 0.023 \text { (stat) } \pm 0.012 \text { (syst) }
\end{aligned}
$$

Or, more importantly, constraining $\operatorname{Re}\left\{\eta_{+-0}\right\}=0.0016$,

$$
\begin{aligned}
& \operatorname{Re}\left\{\eta_{+-0}\right\}=-0.0016 \\
& \operatorname{Im}\left\{\eta_{+-0}\right\}=-0.021 \pm 0.025 \text { (stat) } \pm 0.012 \text { (syst) }
\end{aligned}
$$

Here I quote the $\chi^{2}$ method results where one takes full advantage of the cancellation of systematic effects through the formulation of equation 9.26 where the acceptance and normalization enter as a ratio of downstream to upstream data. 


\section{Chapter Ten}

\section{Conclusions}

\subsection{The Result}

A proper time ratio plot (similar to that of Figure 1.5 for $\eta_{+-0}=\eta_{+-}$) of the downstream over the upstream data, for the east and west beams together, is shown in Figure 10.1. The expected curve for $\eta_{+-0}=\eta_{+-}=0.0016$ is also shown in Figure 10.1 .

The resultant measurement from this analysis was,

$$
\begin{aligned}
& \operatorname{Re}\left\{\eta_{+-0}\right\}=-0.229 \pm 0.046 \text { (stat) } \pm 0.023 \text { (syst) } \\
& \operatorname{Im}\left\{\eta_{+-0}\right\}=0.021 \pm 0.023 \text { (stat) } \pm 0.012 \text { (syst) }
\end{aligned}
$$

Or, more importantly, constraining $\operatorname{Re}\left\{\eta_{+-0}\right\}=0.0016$,

$$
\begin{aligned}
& \operatorname{Re}\left\{\eta_{+-0}\right\}=-0.0016 \\
& \operatorname{Im}\left[\eta_{+-0}\right\}=-0.021 \pm 0.025 \text { (stat) } \pm 0.012 \text { (syst) }
\end{aligned}
$$

The results from this experiment are plotted in Figure 10.2 along with the results of all other experiments from 1972 to the present (given in Table 1.1). Figure 10.3 has the results for $\operatorname{Im}\left\{\eta_{+-0}\right\}$ if $\operatorname{Re}\left\{\eta_{+-0}\right\}$ is constrained to be equal to $\operatorname{Re}\left\{\eta_{+-}\right\}$. The values for $\operatorname{Im}\left\{\eta_{+-0}\right\}$ are plotted versus the year of publication with the results of E621 shown for the year 1990. The improvement over previous results is about an order of magnitude. The next step is to improve the measurement of $\eta_{+-0}$ to the levels of the present measurements of $\eta_{+-}$. 


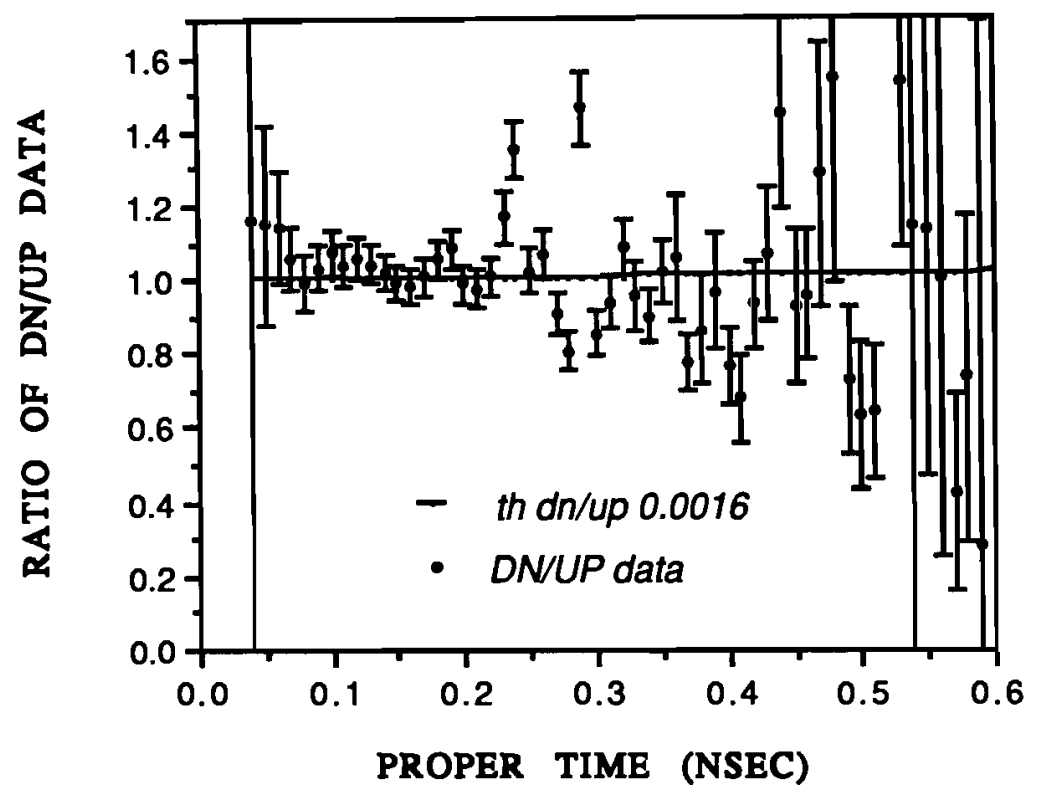

Figure 10.1 Proper Time Ratio of Data: Both East and West Beams

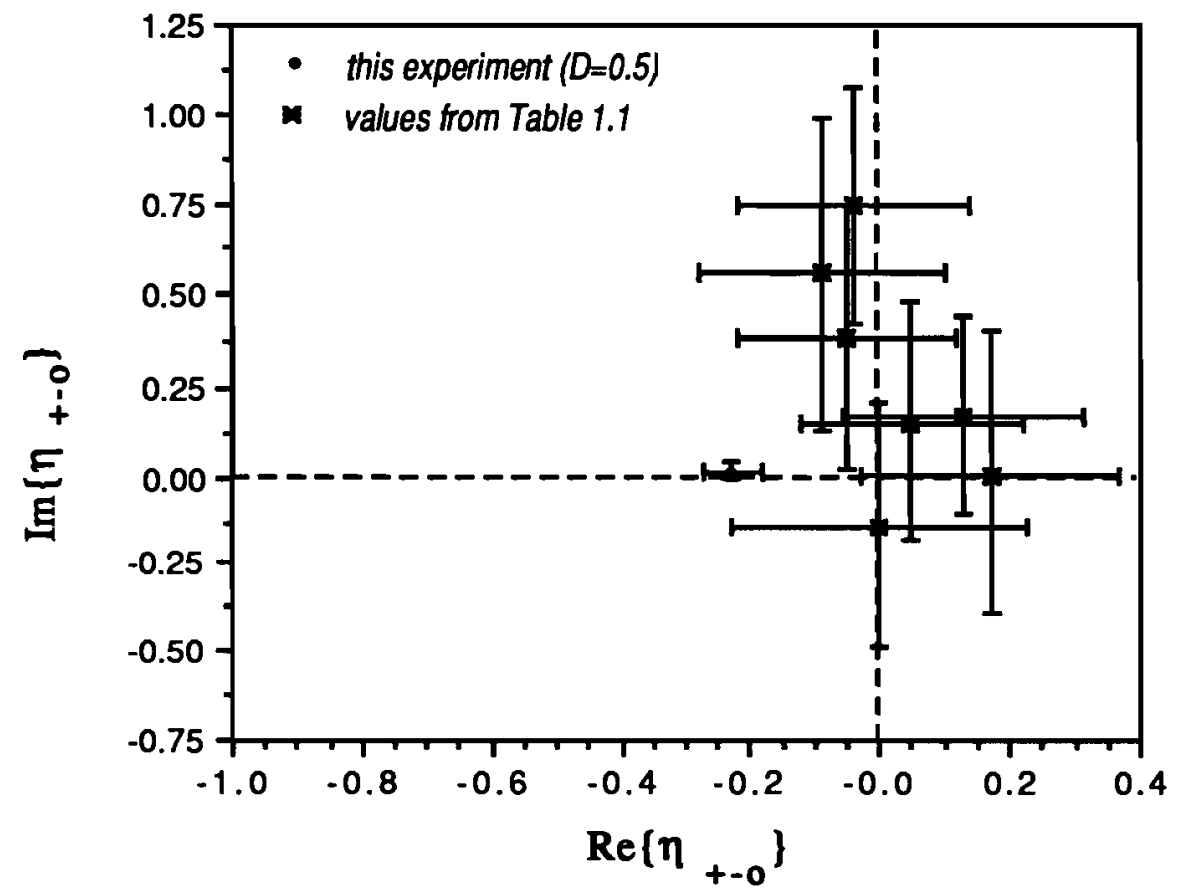

Figure 10.2 Graph of Experimental Values of $\eta_{+-0}$ from 1972 to Present with this Experiment's results 


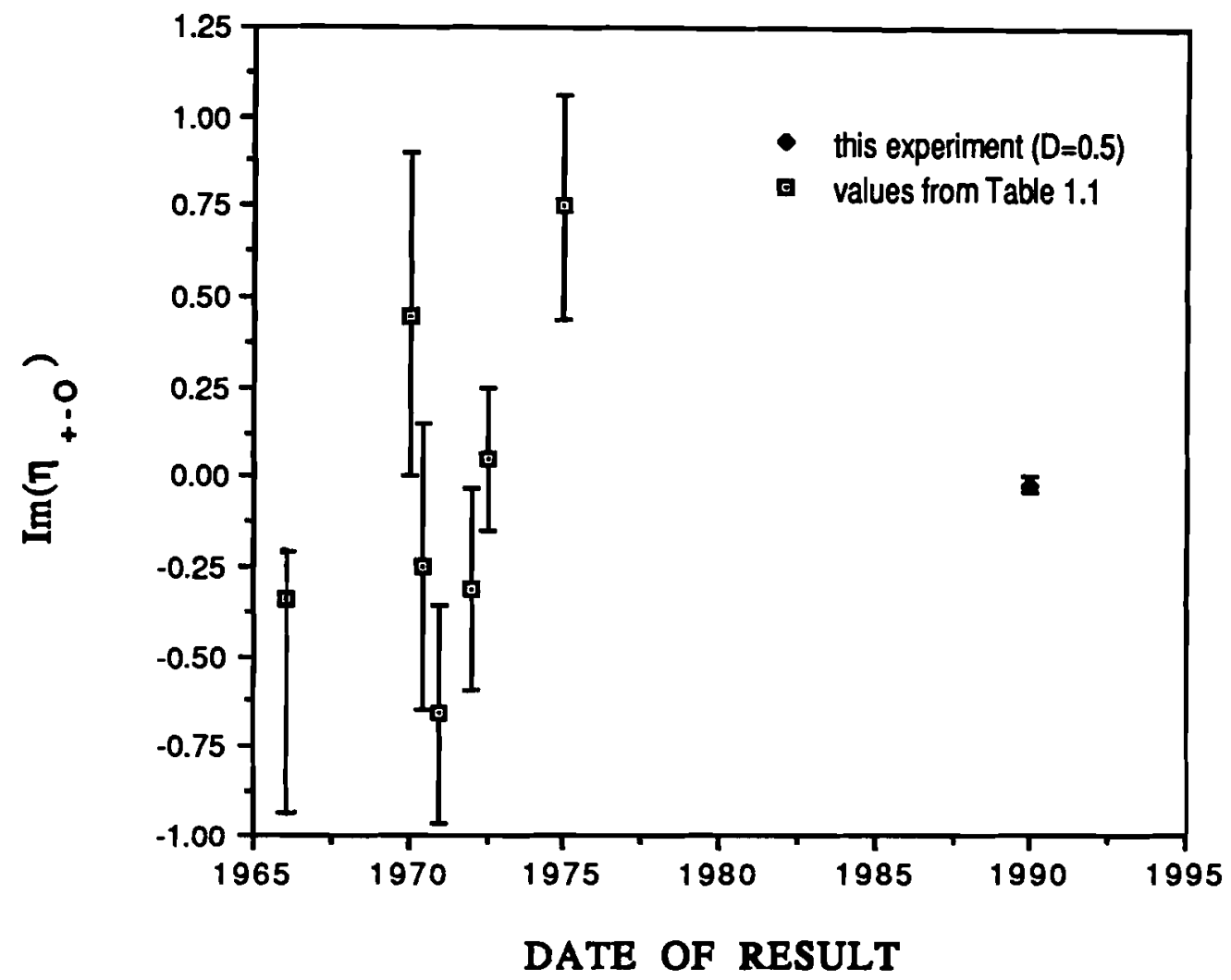

Figure 10.3 Graph of Experimental Values of $I m\left[\eta_{+-0}\right]$ with this Experiment's results for $R e\left[\eta_{+-0}\right]$ Constralned to Zero

\subsection{Implications of the Result}

The discovery of CP symmetry violation in 1964 shattered the illusion concerning the fundamental nature of these invariances. Questions were raised about the origin of CP symmetry violation and the origin of the associated violation of $T$ invariance. These questions have yet to be satisfactorily answered despite an enormous experimental and theoretical effort.

The neutral $\mathrm{K}$ mesons have very special properties that make it possible to establish the existence of a small violation of CP symmetry $\left(\sim 10^{-3}\right)$. That in turn provides evidence for the violation of $T$ invariance. The observed CP symmetry violating effect in $\mathrm{K} \rightarrow 2 \pi$ decay is thought to be primarily due to indirect causes 
184

associated with the mass matrix and thus the chances of observing motion reversal violation, associated with the direct measurement of the CP symmetry violating interaction in the decay, seems remote.

In $\mathrm{K} \rightarrow 2 \pi$ decay the mass matrix term of $\mathrm{CP}$ symmetry violation $(\approx \epsilon)$ is the same as that for $\mathrm{K} \rightarrow 3 \pi$ decay. Yet the decay term associated with the non-invariance of CP is different. It is possible that it could be unexpectedly large. Using the constraint $\operatorname{Re}\left\{\eta_{+-0}\right\} \approx \operatorname{Re}\left\{\eta_{+}\right\}=0.0016$, the best measurement before this experiment was $\operatorname{Im}\left\{\eta_{+-0}\right\}=0.75+(0.27)-(0.35)$ with $\operatorname{Re}\left\{\eta_{+-0}\right\}=0.0016$. Although no $C P$ symmetry violation was seen in this experiment, the limit was decreased an order of magnitude to $\operatorname{Im}\left\{\eta_{+-0}\right\}=-0.021 \pm 0.025$ (stat) \pm 0.012 (syst) with $\operatorname{Re}\left\{\eta_{+-0}\right\}=0.0016$. Although this result can not distinguish between the Superweak model, the K-M model, the Weinberg-Higgs model or the L-R Symmetric model all which predict $\eta_{+-0} \leqq 10^{-3}$, there are no large surprises in the $\mathrm{K} \rightarrow 3 \pi$ system.

\subsection{The Experimental Technique/Future Measurements}

The resultant $\eta_{+-0}$ measurement and the systematic measurements from Chapter 9 tell of the strengths and weaknesses of this experiment and method of analysis. The experimental technique worked well in several aspects. The dual beam-dual target method was invaluable. The Monte Carlo simulation program did not have to be perfect because the upstream target data could be used to correct for the remaining biases. This was necessary in the final measurement of $\eta_{+-0}$. Also having two beams and thus two seperate measurements of $\eta_{+-0}$ is an excellent check of systematic uncertainties in the final result.

The $\mathrm{K} \rightarrow 2 \pi$ data sample, taken simultaneously with the $\mathrm{K} \rightarrow 3 \pi$ sample, provided a necessary constraint for the number of kaons produced on the target. The $\mathrm{K} \rightarrow 2 \pi$ sample also provided information about the accuracy of the Monte Carlo simulation program and the final analysis technique due to the accuracy with which the $K_{s}$ lifetime and $\eta_{+-}$are known.

The special trigger processors, CMR, MLU and CGF, were very effective at removing $\Lambda, \Xi$, and $K \rightarrow 2 \pi$ decays from the $K \rightarrow 3 \pi$ sample at the trigger level. The concept and application of removing events at the trigger level whose charged momentum ratio differed from one (i.e. $P_{+} / P_{-}>3.0$ or $P_{+} / P_{-}<0.3$ ), as in the $C M R$ 
and MLU trigger processors, removed $85 \%$ if the lambda and cascade decays. Unfortunately using both the MLU and the CMR triggers lost too many events due to the noise and inefficiencies in the elements upon which they relied to measure the ratio. Since they both measured the same value, only the more efficient CMR trigger should have been used. The other trigger processor, the CGF trigger processor, was very effective in identifying two or more neutral showers in the lead glass array and thereby removing $\mathrm{K} \rightarrow 2 \pi$ decays from the $\mathrm{K} \rightarrow 3 \pi$ trigger sample.

The theoretical calculations of CP symmetry violating parameters contain too many unknowns. The only experimental measurement to date of $\mathrm{CP}$ symmetry violation are in the $\mathrm{K} \rightarrow 2 \pi$ decay modes. Thus a measurement of $C P$ symmetry violation in an alternate decay mode, $K \rightarrow 3 \pi$, or in a different system, the $B \bar{B}$ system, would be of great interest to theorists. Can a non-zero measurement of $\eta_{+-0}$ be made with an improved spectrometer and data acquisition system using the techniques of this analysis? The answer is not clear. Certainly a better understanding the dilution factor, $\mathbf{D}_{K K}$, is needed. With a larger sample of $K \rightarrow 2 \pi$ decays recorded simultaneously with the $\mathrm{K} \rightarrow 3 \pi$ decays, $D_{K K}$ could be measured. There is also the difference between the results from the east and west beams. I believe this difference is due to an inaccurate measurement of this normalization for the west beam. If this is the source of the difference and it can be corrected with further analysis, then the limitations at this level are statistical. Projecting the results of this experiment, a factor of 1000 in statistics gives a result with an uncertainty of $\sim 0.0006$. Assuming $\eta_{+-0} \approx \eta_{+-} \approx 0.002$, the resultant measurement would be a 3-4 standard deviation effect.

Some obvious improvements can be made to the spectrometer if one considers this same basic design for a future measurement of $\eta_{+-0}$. The radiation damage in the lead glass array lost valuable data acquisition time, (when the damaged blocks were replaced and recalibrated every three weeks), and made simulation of the neutral showers difficult. $60 \%$ of the events showered in a block next to the hole in the center of the array. These events had poor resolution and some $(15 \%)$ were eliminated from the final data sample since they were impossible to simulate accurately. Thus, for the neutral portion of the experiment, a calorimeter which would not exhibit radiation damage should be used. The calorimeter could be made 


\section{6}

larger and moved further downstream in order to allow the photons to seperate further and eliminate the problems of poor resolution near the neutral beam in the center. At most these improvements could provide a factor of 2 in the data sample size.

The charged spectrometer could be improved also. The MWPC rates limited our beam intensity. Chambers which could perform well at an increased intensity is a direct improvement, assuming an improved data acquisition system could accomodate the increased event rate. Also increased chamber efficiencies, through more careful montioring, would increase the efficiency of the CMR trigger processor or a similar trigger processor. The smallness of the analysis magnet aperature lost $40 \%$ of the events (according to the Monte Carlo) because the photons hit the magnet. This aperature could be made larger. The suggested improvements are given in Table 10.4 .

(1) more $K \rightarrow 2 \pi$ events

(2) larger, radiation damage-free calorimeter (further downstream)

(3) larger analysis magnet gap and field (to seperate the charged \& neutral showers more)

(4) faster data acquisition system (necessary for higher rate experiment)

(5) upstream chambers capable of taking rates of $10 \mathrm{Mhz}$ and closer monitoring of the chamber efficiencies for those chambers used in a trigger processor

(6) a CMR (or similar) trigger processor

(7) a GCF (or similar) trigger processor

Table 10.4 Possible Design Improvements

If the entire data sample form E621 were analyzed, the statistical uncertainty would be $\sim 0.01$. For $10^{3}$ times the statistics the uncertainty in the measurement of $\eta_{+-0}$ would be $\sim 0.0003$, most likely small enough to observe CP symmetry violation. Assuming that data acquisition and chamber technology can accomodate an order of magnitude in intensity $\left(3 \times 10^{12}\right.$ protons on target), a factor of 100 is needed elsewhere. The improvements listed in Table 10.4 could probably provide a factor of 10 in trigger efficiency. Another factor of 2 could possible come from looser data selection criteria due to a better understanding of the apparatus, but the last factor 
of 5-10 in statistics is very hard to obtain with present technology and this experimental design. 


\section{Appendix A}

\section{Complete Enclosure and Collimator Diagrams}

Enclosure PC1:

PLAN VIEW:
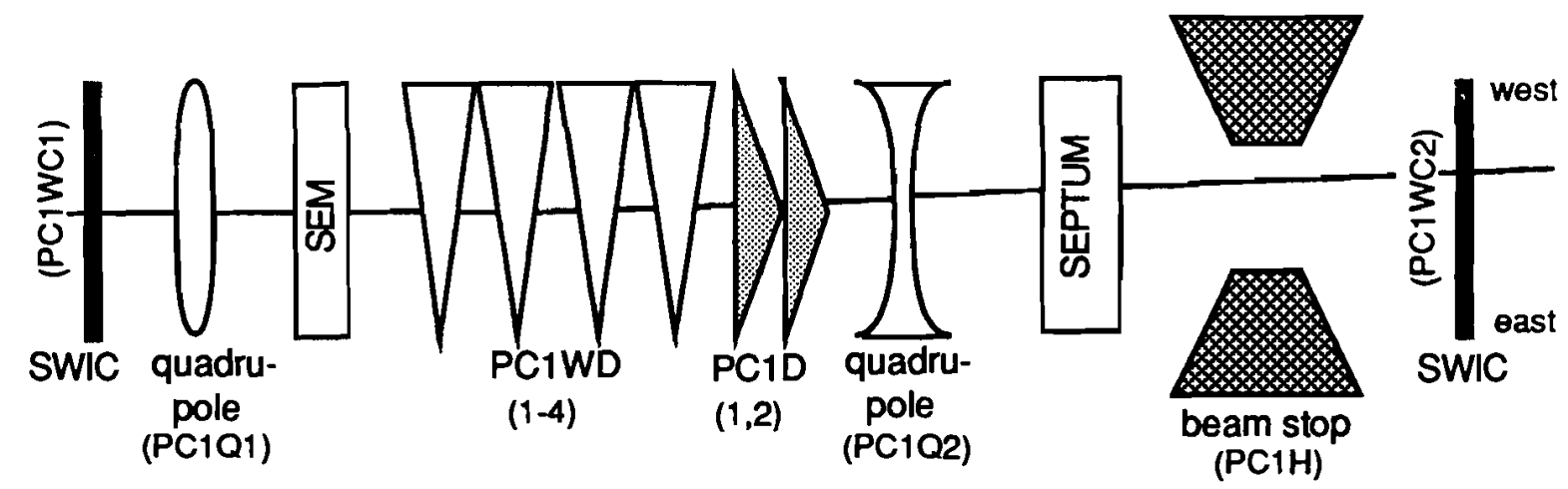

$\begin{array}{lr}\text { Device } & \text { Z Position } \\ \text { PC1WC1 } & -685.0 \\ \text { PC1Q1 } & -682.0 \\ \text { SEM } & -680.0 \\ \text { PC1WD(1) } & -678.5 \\ (2) & -675.0 \\ (3) & -672.0 \\ (4) & -668.5 \\ \text { PC1D(1) } & -665.0 \\ (2) & -661.5 \\ \text { PC1Q2 } & -660.0 \\ \text { Septum } & -655.5 \\ \text { PC1H } & -651.0 \\ \text { PC1WC2 } & -649.5 \\ \text { end of PC1 } & -647.5\end{array}$
Key: dipole magnet
$\nabla D$ quadrupole magnet $0 \pi \mathrm{T}$
SWIC

Enclosure PC1 (with dimensions) 
Enclosure PC2:

PLAN VIEW:

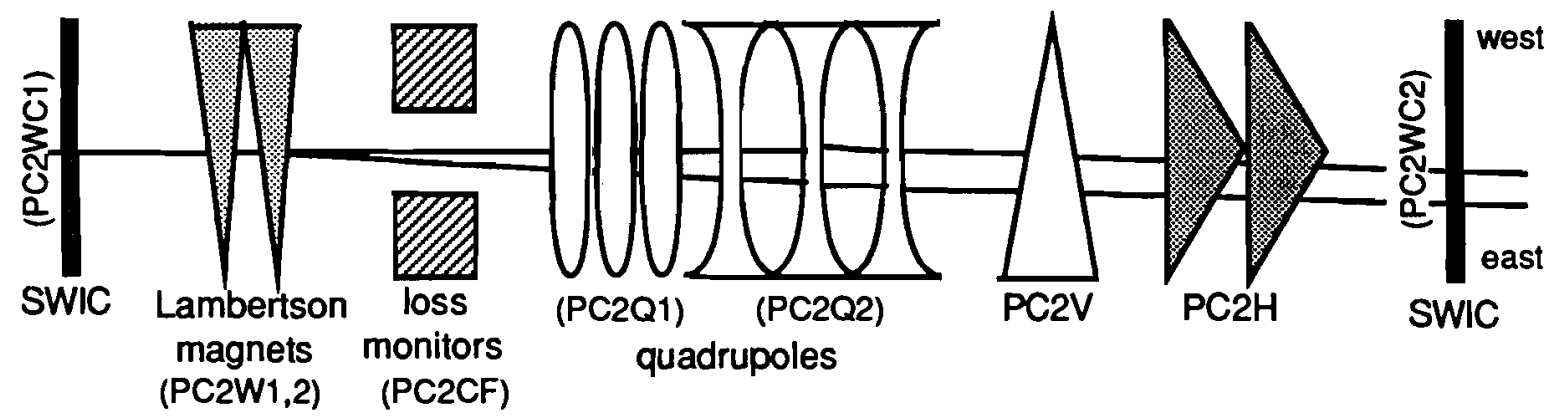

$\begin{array}{lc}\text { Device } & \text { ZPosition (m) } \\ \text { PC2WC1 } & -120.0 \\ \text { PC2W1 } & -114.0 \\ \text { PC2W2 } & -111.0 \\ \text { PC2F } & -107.5 \\ \text { PC2Q1(1) } & -103.5 \\ \text { (2) } & -100.5 \\ \text { (3) } & -97.0 \\ \text { PC2Q2(1) } & -93.5 \\ \text { (2) } & -90.0 \\ \text { (3) } & -86.5 \\ \text { PC2V } & -84.5 \\ \text { PC1H(1) } & -82.5 \\ \text { (2) } & -81.5 \\ \text { PC2WC2 } & -80.5 \\ \text { end of PC2 } & -80.0\end{array}$

$\begin{array}{ll}\text { Key: dipole magnet } & \nabla D \\ \text { quadrupole magnet } & 0 \frac{1}{\text { SWIC }}\end{array}$

Flgure A.2 Enclosure PC2 (with dimensions) 
Enclosure PC3:
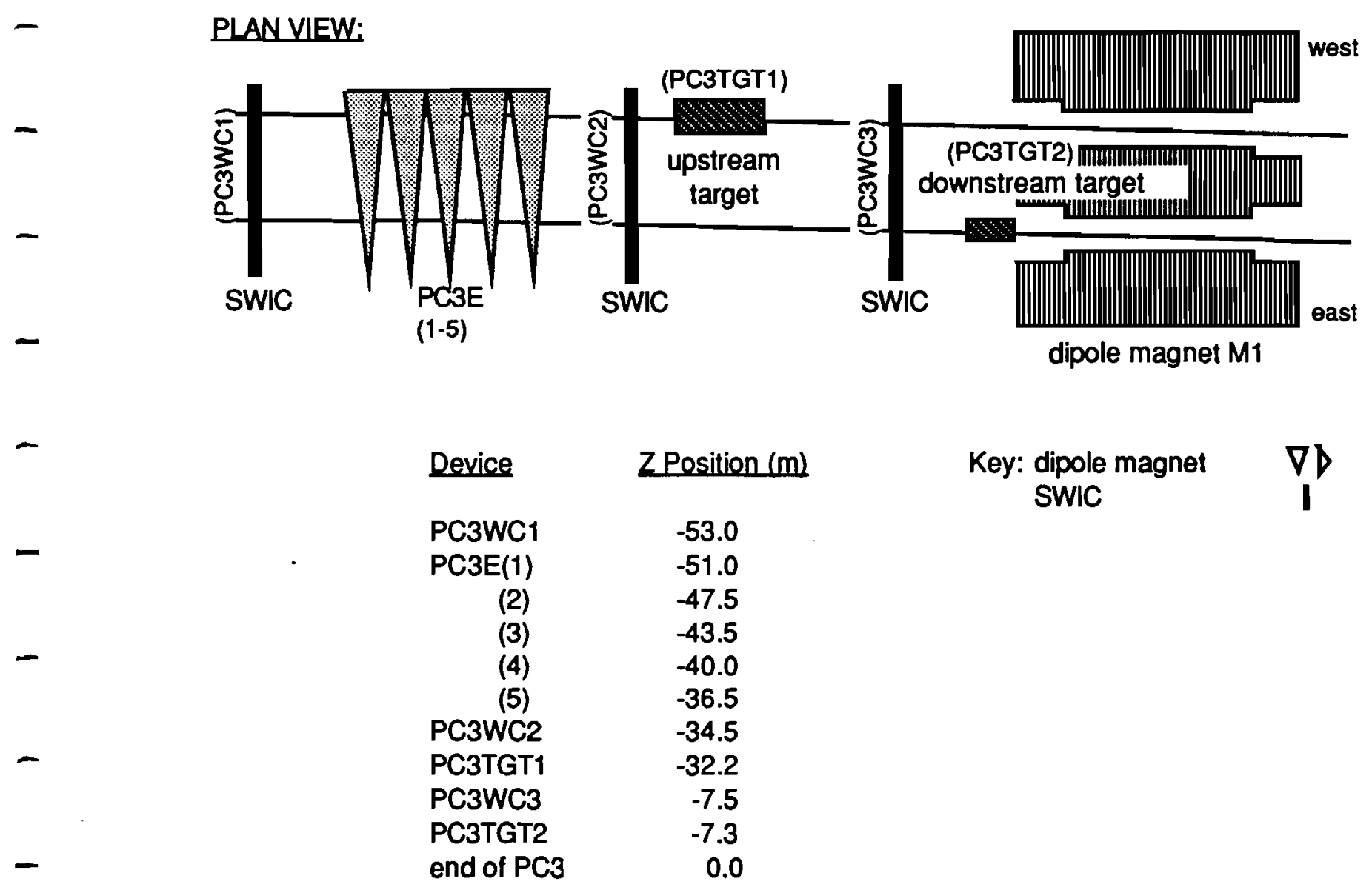

Flgure A.3 Enclosure PC3 (with dimensions) 
PLANVIEW

west

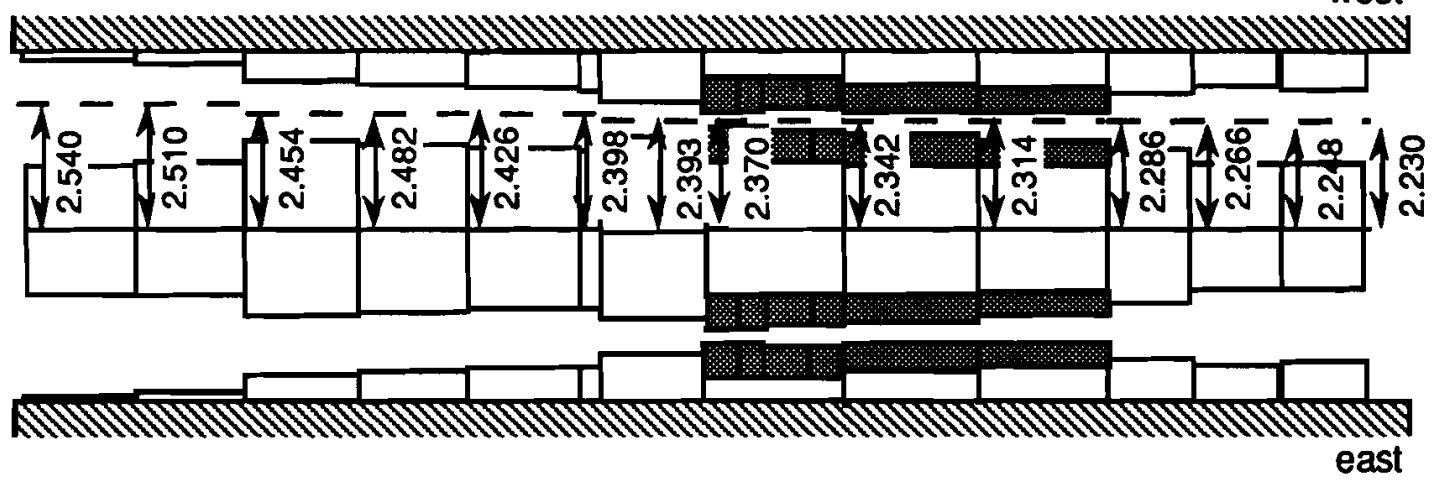

ELEVATION VIEW

up

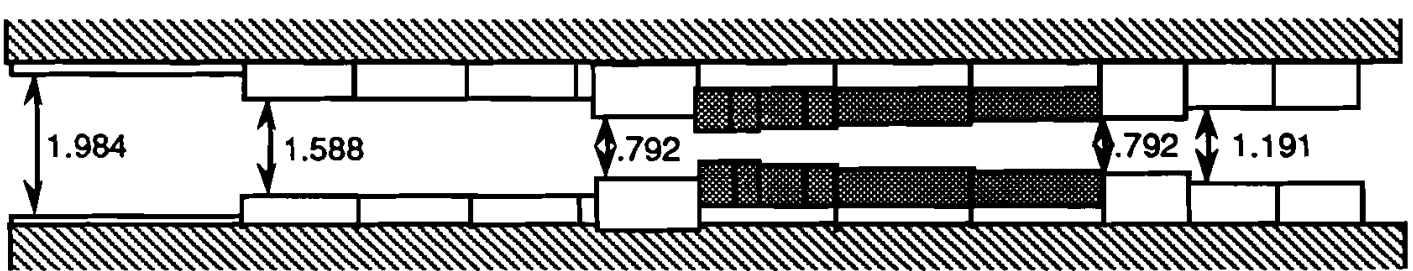

down

(not drawn to scale)

Dipole Magnet M1

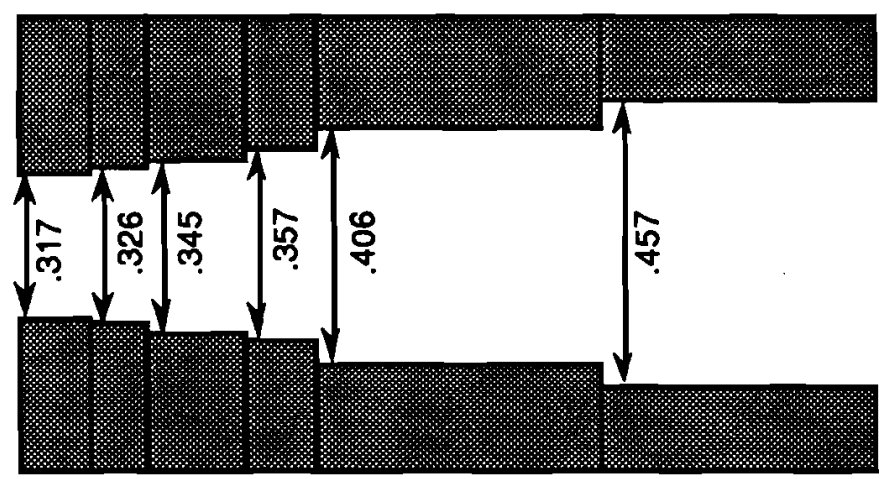

Close-up of Defining Section:

Figure A.4 The Collimator (with dimensions given In cm) 


\section{Appendix B}

\section{Radiation Lengths of Material in Experimental Apparatus}

Table B.1 Radiation Lengths of Material:

\begin{tabular}{|c|c|c|}
\hline Element & Radiation Length & Location(m) \\
\hline $1 \mathrm{~V} 1$ & 0.004 & 0.845 \\
\hline $2 \mathrm{DK}$ & 0.004 & 19.50 \\
\hline 3 window & 0.0007 & 22.58 \\
\hline 4 air & 0.0021 & 23.218 \\
\hline $5 \mathrm{Cly}$ & 0.0009 & 23.219 \\
\hline $6 \mathrm{C} 1 \mathrm{x}$ & 0.0009 & 23.229 \\
\hline 7 air & 0.0013 & 23.633 \\
\hline 8 window & 0.0003 & 23.634 \\
\hline 9 helium & 0.0005 & 26.496 \\
\hline 10 window & 0.0003 & 26.497 \\
\hline 11 air & 0.0013 & 26.888 \\
\hline $12 \mathrm{C} 2 \mathrm{u}$ & 0.0009 & 26.889 \\
\hline $13 \mathrm{C} 2 \mathrm{v}$ & 0.0009 & 26.899 \\
\hline 14 air & 0.0012 & 27.278 \\
\hline 15 window & 0.0003 & 30.141 \\
\hline 16 helium & 0.0005 & 30.141 \\
\hline 17 window & 0.0003 & 30.142 \\
\hline 18 air & 0.0013 & 30.527 \\
\hline 19 C3y & 0.0009 & 30.528 \\
\hline $20 \mathrm{C} 3 \mathrm{x}$ & 0.0009 & 30.538 \\
\hline 21 air & 0.0012 & 31.231 \\
\hline 22 window & 0.0003 & 31.232 \\
\hline 23 helium & 0.0005 & 33.711 \\
\hline
\end{tabular}




\begin{tabular}{lcc} 
Element & Radiation Length & Location(m) \\
\cline { 2 - 3 } 24 helium & 0.0005 & 35.573 \\
25 window & 0.0003 & 35.574 \\
26 air & 0.0013 & 36.607 \\
27 C4y & 0.0009 & 36.608 \\
28 C4x & 0.0009 & 36.618 \\
29 air & 0.0012 & 37.310 \\
30 window & 0.0003 & 37.311 \\
31 helium & 0.0005 & 40.935 \\
32 window & 0.0003 & 40.936 \\
33 air & 0.0013 & 41.649 \\
34 C5y & 0.0009 & 41.650 \\
35 C5x & 0.0009 & 41.660 \\
36 air & 0.0012 & 42.300 \\
37 A hodoscopes $(1)$ & 0.0162 & 42.301 \\
38 A hodoscopes(2) & 0.0162 & 42.352 \\
39 air & 0.0012 & 42.729 \\
40 window & 0.0003 & 42.730 \\
41 helium & 0.0005 & 46.964 \\
42 window & 0.0003 & 46.965 \\
43 air & 0.0013 & 47.348 \\
44 B hodoscopes(1) & 0.0162 & 47.349 \\
45 B hodoscopes(2) & 0.0162 & 47.400 \\
46 air & 0.0013 & 48.193 \\
47 C6y & 0.0009 & 48.194 \\
48 C6x & 0.0009 & 48.204
\end{tabular}




\section{Appendix C}

\section{Collimator Produced Kaon Background}

Ideally the final data samples of $\mathrm{K} \rightarrow 2 \pi$ and $\mathrm{K} \rightarrow 3 \pi$ decays consisted of kaons, produced in one of the targets, which travelled through the collimator into the decay volume. The background to be eliminated was kaons produced in the collimator. Most of these events were removed by requiring all the final $K_{3 \pi}$ and $K_{2 \pi}$ events to have a decay vertex and momentum vector which fell within the angular phase space of events from the targets as determined by the Monte Carlo simulation program (see Table 8.2).

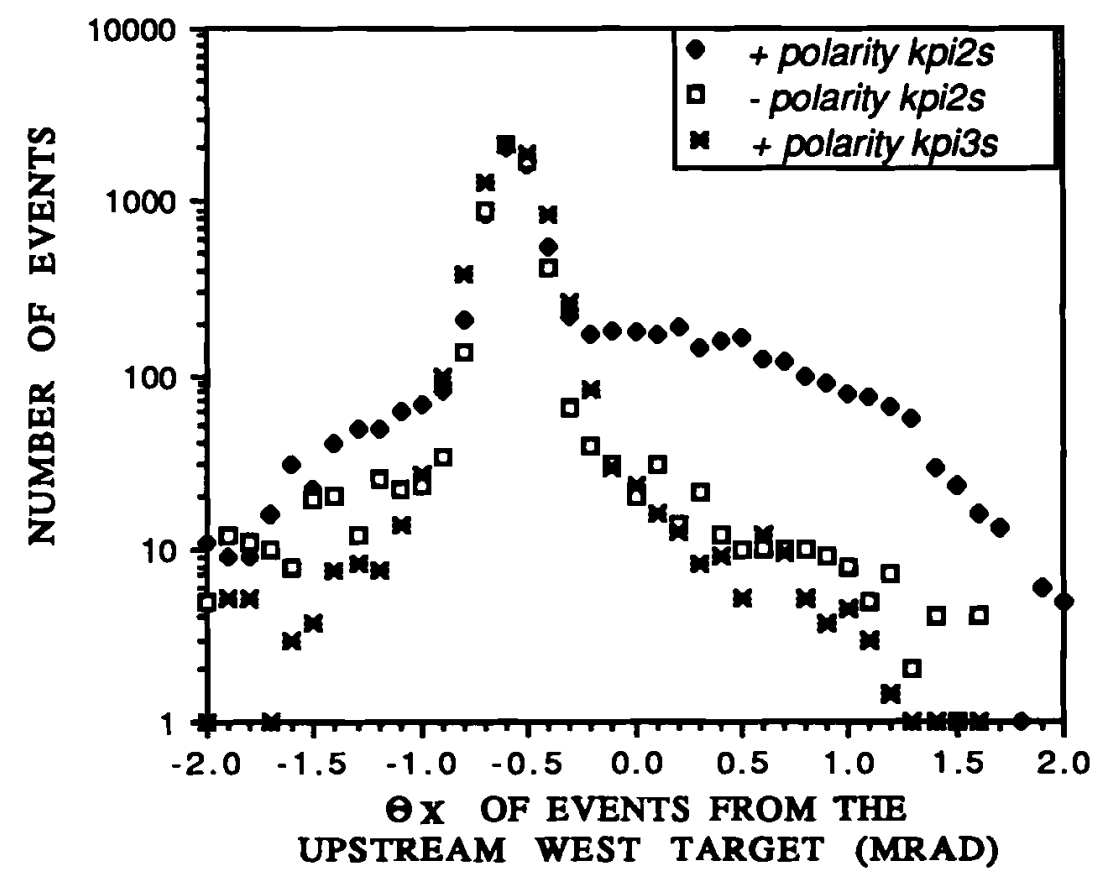

Figure C.1 Neutral Beam Angle in $X$ for Upstream West: Kaon Backgrounds

The $\mathrm{K}_{2 \pi}$ upstream west target sample had clear collimator production, resulting in a tail in the $x$ beam angle distribution, $\Theta_{x}$. This tail disappeared if the 
current in magnet $M 1$ was reversed, indicating that it came from residual proton beam or spray entering the collimator and producing kaons off the inside wall. Due to the $\mathrm{K}_{\mathrm{s}} / \mathrm{K}_{\mathrm{l}}$ lifetime ratio this effect negligible in the $\mathrm{K}_{3 \pi}$ sample. Figure $\mathrm{C.1}$ shows the $\Theta_{x}$ distribution for the upstream west data for both polarities of magnet $M 1$ for $\mathrm{K}_{2 \pi}$ data and the positive polarity only for $\mathrm{K}_{3 \pi}$ data. All other targets had background levels similar to that of the minus polarity data in Figure C.1.

The level of collimator produced kaon contamination had to be known since the measurement of $\eta_{+-0}$ required an accurate measurement of the number of kaons produced in each target. A Monte Carlo simulation of kaons produced near the center of the defining section of the collimator provided beam phase space distributions of collimator produced kaons. Figure C.2 shows the agreement between Monte Carlo and data for the upstream west target for $\mathrm{K}_{2 \pi}$ events.

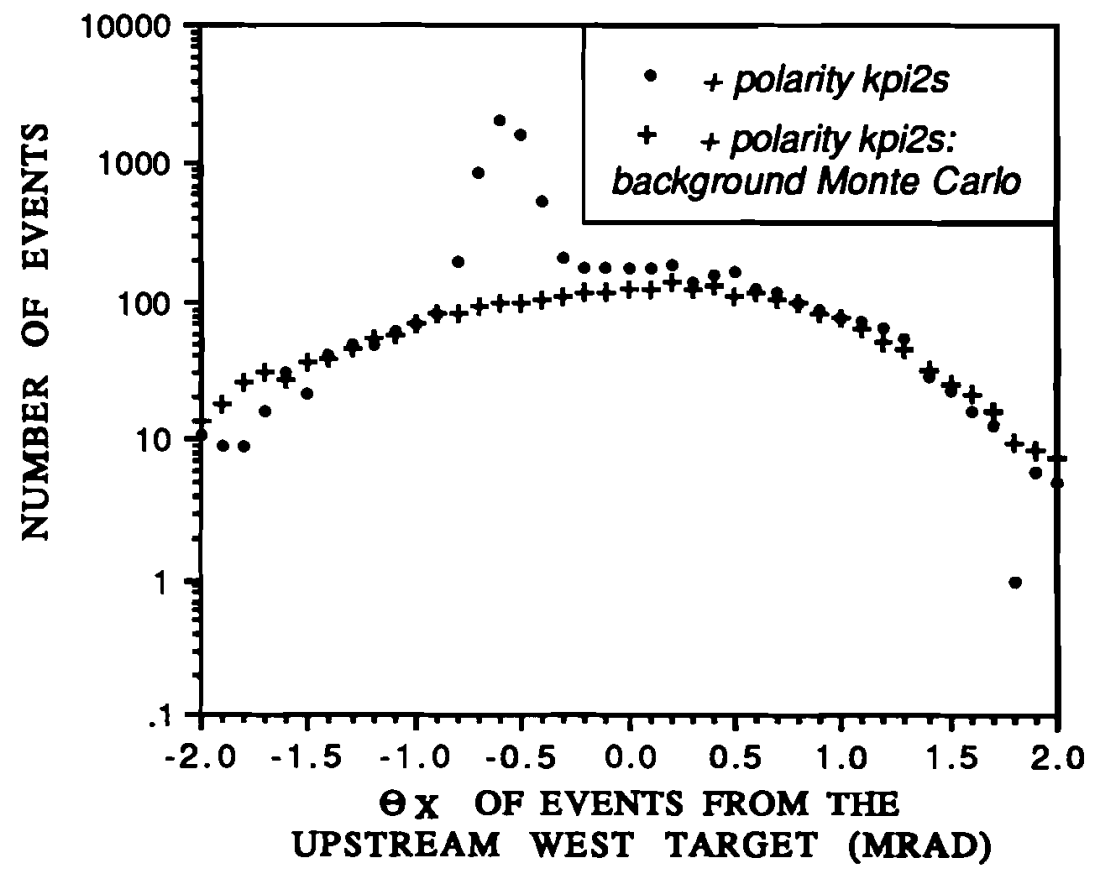

Figure C.2 Simulation of Collimator Kaon Background In Upstream West $\mathrm{K} \rightarrow 2 \pi$ Decay

Matching the data tails in the beam distributions to a collimator produced kaon Monte Carlo provided good estimates of the amount of background remaining in the 
final data samples. These background Monte Carlo events were then normalized and subtracted from the data. Table C.1 shows the level of contamination of the events in the final $K_{3 \pi}$ and $K_{3 \pi}$ data samples with a kaon momentum cut of $130 \mathrm{GeV}$ to 350 GeV.

\begin{tabular}{|c|c||c|}
\hline$\underline{\text { Target }}$ & $\begin{array}{c}\mathrm{K} 2 \pi \\
\text { Background }\end{array}$ & $\begin{array}{c}\mathrm{K} 3 \boldsymbol{\pi} \\
\text { Background }\end{array}$ \\
\hline Downstream West & $0.8 \%$ & $1.1 \%$ \\
\hline Downstream East & $0.4 \%$ & $0.8 \%$ \\
\hline Upstream West & $1.5 \%$ & $0.2 \%$ \\
\hline Upstream East & $0.4 \%$ & $0.2 \%$ \\
\hline
\end{tabular}

Table C.1 Percentages of Collimator Produced Kaon Background 


\section{Appendix D}

\section{The Dilution Factor}

In equation 1.56 the dilution factor $D_{\mathbf{K} \overline{\mathbf{K}}}$ multiplies the interference term containing $\eta_{+-0}$. A measure of $\eta_{+-0}$ becomes a measurement of $D_{K \bar{K}}$ times $\eta_{+-0}$ if $D_{\mathrm{K} \bar{K}}$ is unknown. $D_{K \bar{K}}$ is defined in equation 1.60 as:

$$
D_{K \bar{K}}=\frac{K^{0}-\overline{K^{0}}}{K^{0}+\overline{K^{0}}}=\frac{K^{0} / \overline{K^{0}}-1}{K^{0} / \overline{K^{0}}+1}
$$

where $\mathrm{K}^{\circ}$ and $\overline{\mathrm{K}^{0}}$ are the production fluxes at the target.

The size of $D_{K \bar{K}}$ can be estimated from the production cross-sections for $\mathrm{K}^{+}$and $\mathrm{K}^{-}$ particles. The production mechanics for neutral kaons are similar to those of charged kaons which have been measured several times ${ }^{(44)}$. There is also a prediction by Field and Feynmann ${ }^{(45)}$ for $90^{\circ}$ scattering in the center of mass system shown in Figure D.1. Although E621 was not near $90^{\circ}$ in the center of mass, the $\mathrm{K}^{+} / \mathrm{K}^{-}$production ratio is relatively independent of angle ${ }^{(46)}$. Figure $D .1$ also shows Antreasyans and Johnson's results as a function of Feynmann $X$-radial, where $X$-radial is the normal Feynmann's $X$ compensated for the large targeting angles in Antreasyans's experiment.

In E621, for the final data sample the average Feynmann $X$ was 0.2 . From Figure D. 1 this corresponds to:

$$
\frac{\mathrm{K}^{+}}{\mathrm{K}^{-}} \approx \frac{\mathrm{K}^{o}}{\overline{\mathrm{K}^{o}}} \approx 3
$$

Using equation $D .1$ this gives $D_{\mathrm{KK}}$ as 0.5 . And over the momentum range of $130 \mathrm{GeV}$ to $350 \mathrm{GeV}$ the slope is difficult to determine because of the large errors on the experimental results.

The $\mathrm{K}_{2 \pi}$ data sample could be used to measure $\mathbf{D}_{\mathbf{K} \overline{\mathrm{K}}}$ but with low statistical precision:

$$
\mathrm{D}_{\mathrm{K} \overline{\mathrm{K}}}=-0.48 \pm 0.46 \text { with a } \chi^{2} / \mathrm{DOF}=1.11
$$


Since $\eta_{+-}$and $D_{\mathbf{K K}}$ are the coefficients of a long-lived term in $\eta_{+-}$decay, expanding the data sample to lower momentum provided a better measurement of $D_{K \bar{K}}$. Increasing the momentum range to $80-350 \mathrm{GeV}$ and using the Particle Data Group values for $\eta_{+-}$:

$$
D_{\mathrm{K} \bar{K}}=0.2 \pm 0.1 \text { with a } \chi^{2} / \mathrm{DOF}=1.15 \text {. }
$$

This data sample correponded to an average Feynmann $X$ of 0.19 or $D_{\mathbf{X} \bar{K}}=0.43$ as determined from Figure D.1. Thus the resultant measurement is two standard deviations below the value predicted by Field and Feynmann.

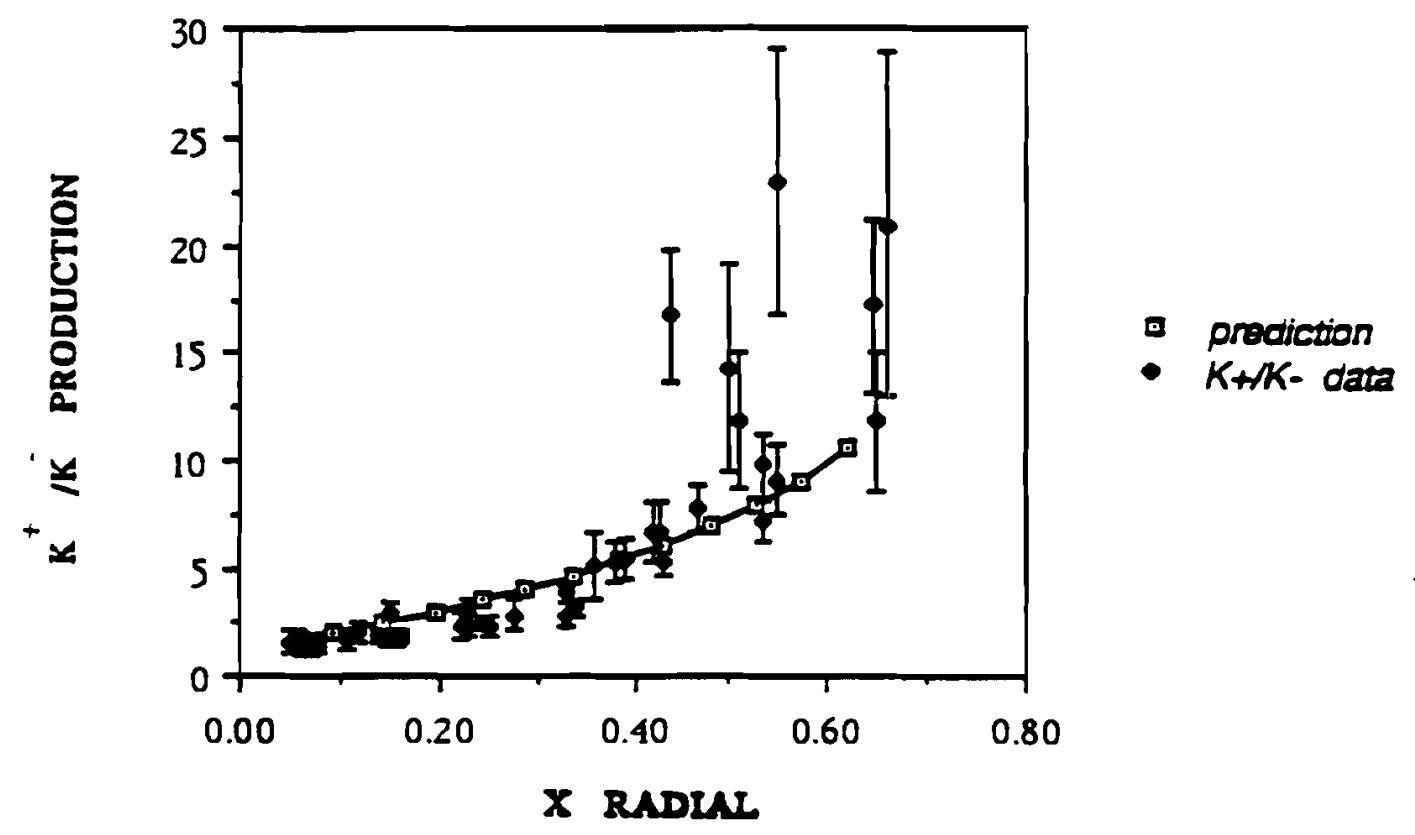

Figure D.1 $\mathrm{K}^{+} / \mathrm{K}^{-}$Production Ratios 


\section{Appendix E \\ The CP-Conserving Decay}

One might worry about the CP-conserving decay $\mathrm{K}_{\mathrm{s}} \rightarrow \pi^{+} \pi^{-} \pi^{0}$ and its effect on E621 results. For a wavefunction of pions in an isospin state $n$, the CP eigenvalue is $(-1)^{n}$. It follows that two pions, which must be in an isospin state $I=0$ or 2 , have a CP eigenvalue of 1 . But by adding a third pion one adds isospin states 1 and 3 and thus the CP eigenvalue -1 also. Fortunately Lee and $W_{u} 47$ have estimated the matrix elements for these CP-conserving decays and discovered that they are strongly suppressed by the angular momentum barrier and the $\Delta \mathrm{I}=1 / 2$ rule.

In addition to these effects, the CP-conserving decay is even less important in E621 since the final measurement averages over the Dalitz plot. The CP-conserving decay amplitude is proportional to $S_{1}-S_{2}$ on the Dalitz plot ${ }^{(48)}$ where $S_{i}$ is defined as:

$$
\mathrm{S}_{i}=\left(\overrightarrow{\mathrm{P}}_{k}-\overrightarrow{\mathrm{P}}_{i}\right)^{2}=\left(m_{k}-m_{i}\right)^{2}-2 m_{k} \mathrm{~T}_{i}
$$

where $m_{k}$ is the kaon mass, $m_{i}$ the pion mass, $\mathrm{T}_{i}$ the pion kinetic energy in the $\mathrm{K}^{\mathrm{o}}$ rest frame and $\overrightarrow{\mathrm{P}}_{k}$ and $\overrightarrow{\mathrm{P}}_{i}$ the kaon and pion four-vectors. Averaging over the Dalitz plot removes this decay from the overall decay rate given that the acceptance is symmetric in $S_{1}$ and $S_{2}$. In E621 symmetric acceptance was acheived by reversing the polarity of the M2,M3 magnet combination periodically. Thus the CP-conserving decay was not a problem. 


\section{Appendix F}

\section{Tabulated Data and Acceptance}

\section{F.1 Monte Carlo-Data Dalitz Distributions}

The vertical axis is the the center of mass momentum of the positively charged pion and the horizontal axis is the momentum of the negatively charged pion. The bins are $4 \mathrm{MeV}$ wide, starting at $(0,0)$ in the upper left hand corner.

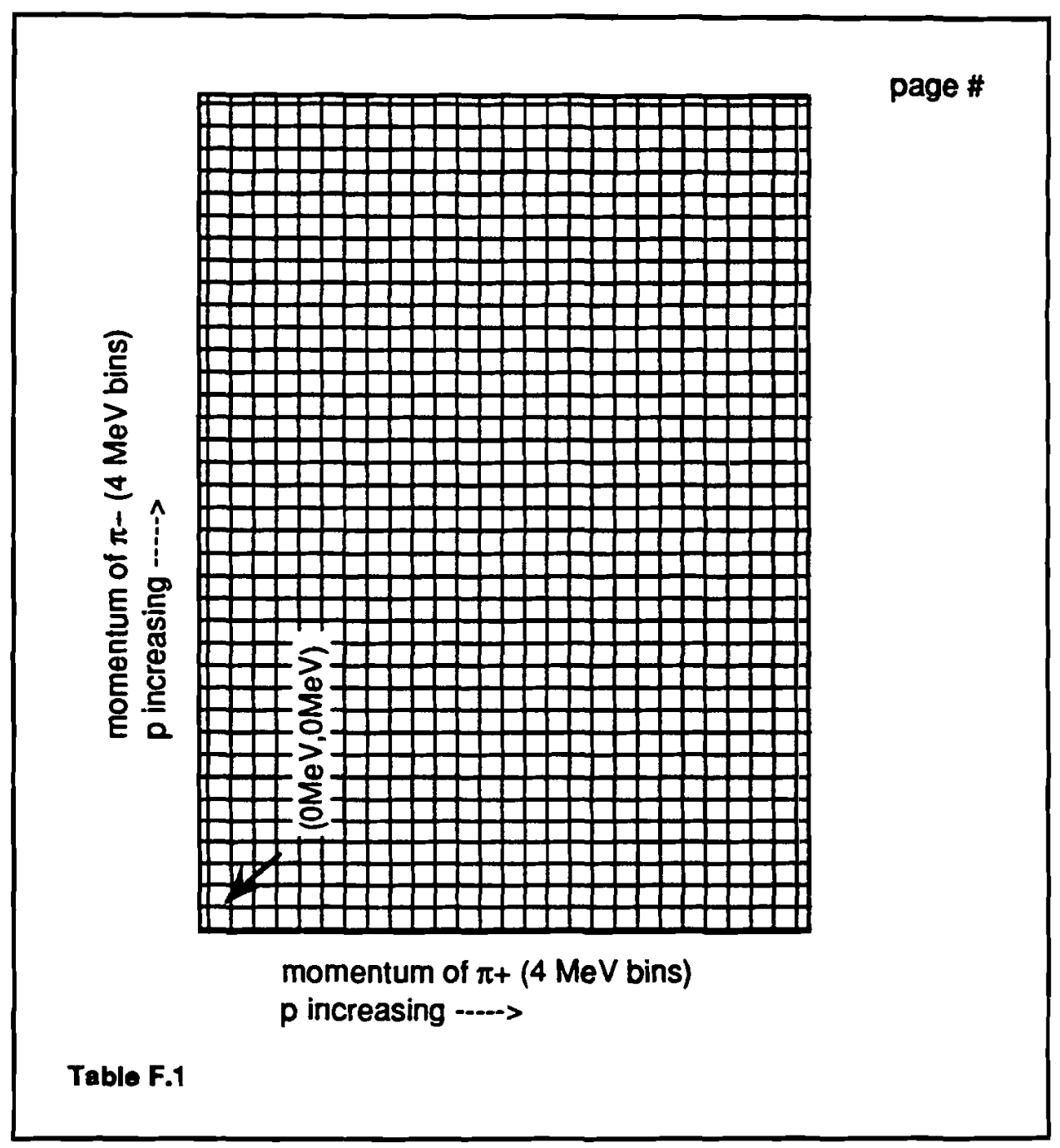




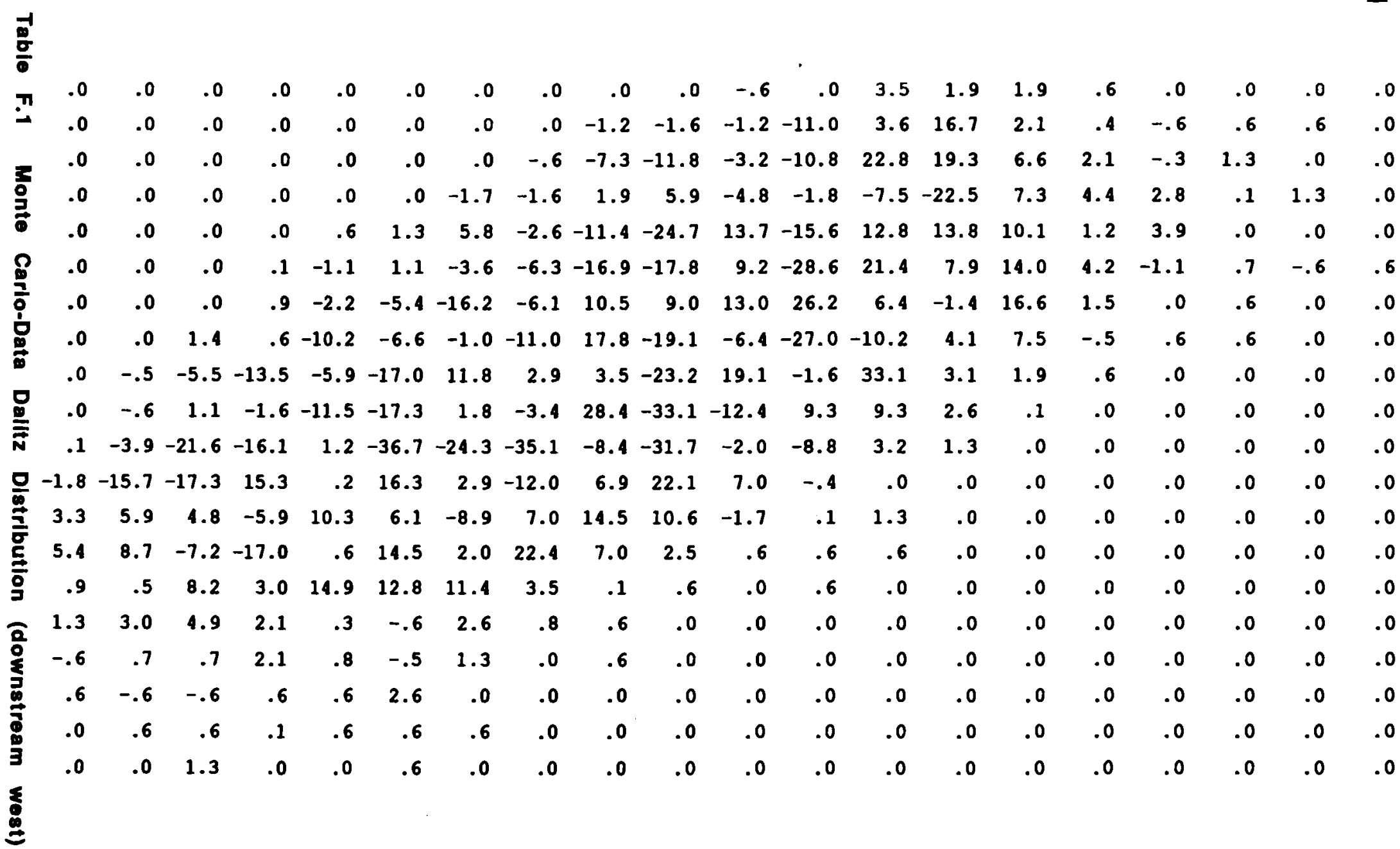




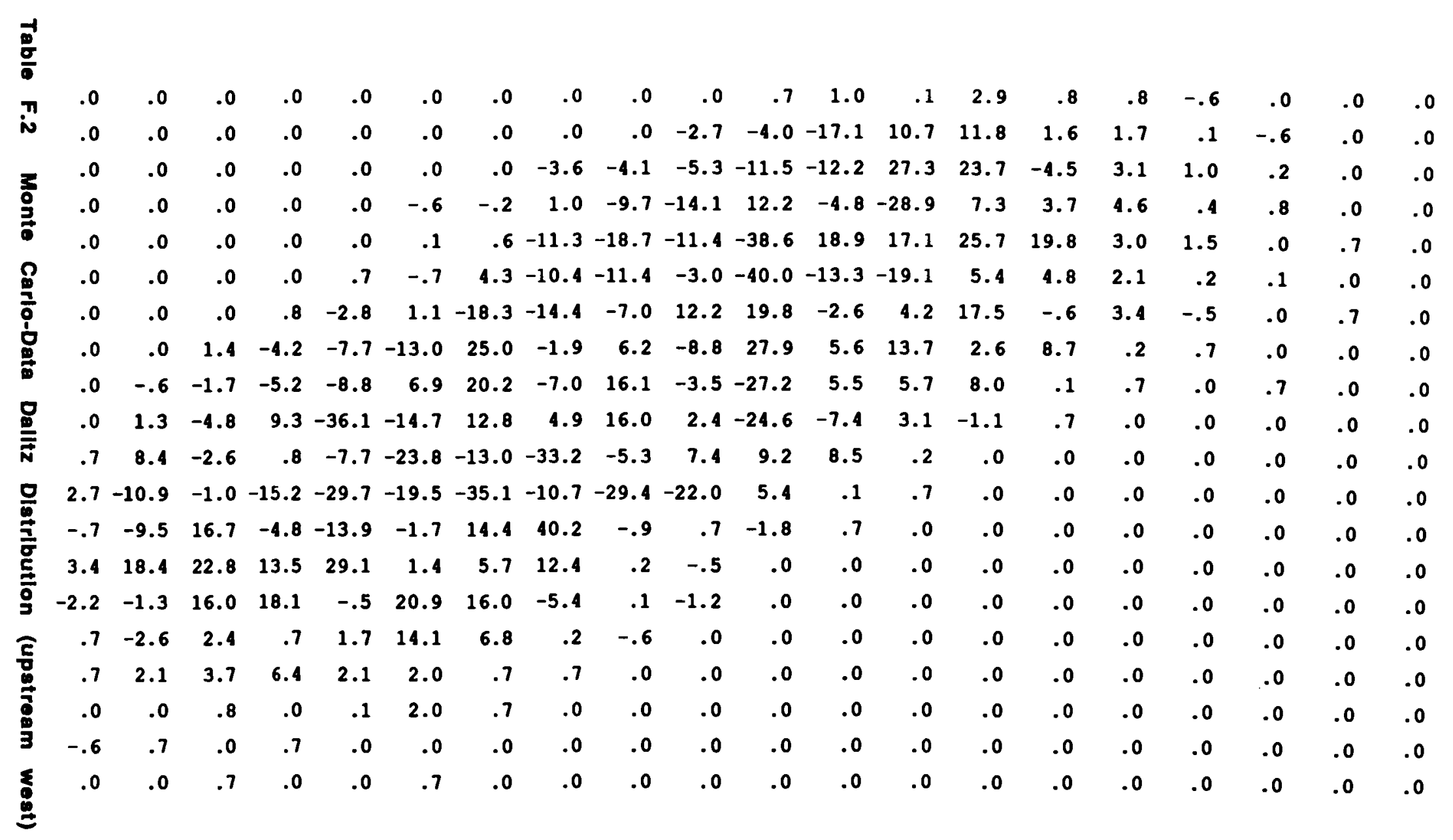


$\pi \quad \begin{array}{lllllllllllllllllllll}0 & .0 & .0 & .0 & .0 & .0 & .0 & .0 & .0 & .0 & .6 & .0 & 1.1 & -1.8 & 1.7 & .6 & .6 & .0 & .0 & .0\end{array}$

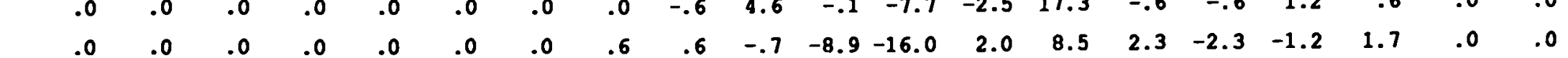

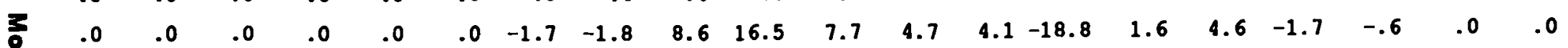

\begin{tabular}{llllllllllllllllllllll}
\hline & .0 & .0 & .0 & .0 & .0 & 1.2 & -.6 & 8.0 & -5.4 & -24.7 & -1.7 & 2.2 & 38.8 & 16.5 & 5.1 & -1.2 & 1.7 & .6 & .0 & .0
\end{tabular}

$\begin{array}{lllllllllllllllllllll}0 & .0 & .0 & .0 & -1.2 & -1.7 & .6 & 9.8 & 8.5 & -12.0 & 23.8 & -.7 & -31.5 & 8.0 & 3.2 & 4.0 & -2.3 & -.6 & -.6 & -.6 & .0\end{array}$

$\begin{array}{llllllllllllllllllllll}8 & .0 & .0 & .0 & -1.7 & -5.2 & -3.6 & -10.7 & 1.4 & 14.0 & 8.6 & 20.1 & .3 & 30.6 & -14.7 & -1.8 & -.6 & .6 & .0 & .0 & .0\end{array}$

\begin{tabular}{llllllllllllllllllllll}
\hline 0 & .0 & .0 & .0 & -1.2 & 1.7 & 21.2 & 1.4 & .6 & -.1 & -3.8 & -6.7 & -3.2 & -9.8 & 6.8 & .0 & -.6 & .6 & .0 & .0 & .0
\end{tabular}

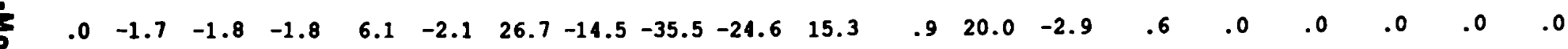

$\begin{array}{lllllllllllllllllllllll} & .0 & 2.3 & 5.7 & 14.2 & -3.9 & -8.1 & -11.7 & 13.1 & 10.7 & -20.2 & 10.6 & -27.6 & -8.2 & 2.3 & -.6 & .0 & .0 & .6 & .0 & .0\end{array}$

$\begin{array}{llllllllllllllllllllll}.0 & -3.5 & -20.5 & 5.4 & 2.3 & -5.4 & 12.5 & -3.3 & -40.4 & -.9 & -2.2 & 7.4 & 6.9 & 2.9 & .6 & .0 & .0 & .0 & .0 & .0\end{array}$

$\begin{array}{llllllllllllllllllllll}8 & -1.2 & -18.1 & 8.3 & 5.9 & -18.0 & 22.4 & 18.3 & 6.6 & -7.2 & 7.1 & 13.8 & 3.5 & 1.2 & .0 & .0 & .0 & .0 & .0 & .0 & .0\end{array}$

$\begin{array}{llllllllllllllllllll}2.9 & -4.8 & -2.7 & -21.4 & 7.5 & 8.0 & -11.1 & -12.7 & -4.3 & 9.8 & 5.8 & .6 & 1.2 & .0 & .0 & .0 & .0 & .0 & .0 & .0\end{array}$

$\begin{array}{llllllllllllllllllll}.6 & 6.3 & 4.4 & -38.0 & -24.1 & 14.7 & -15.3 & 1.0 & -1.8 & 5.2 & .6 & .0 & .0 & .0 & .0 & .0 & .0 & .0 & .0 & .0\end{array}$

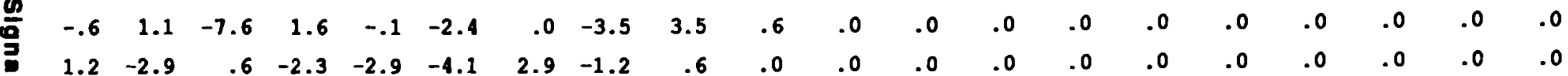

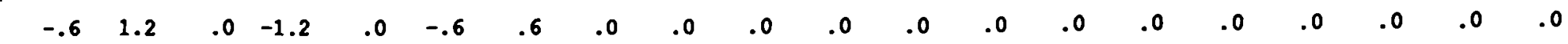

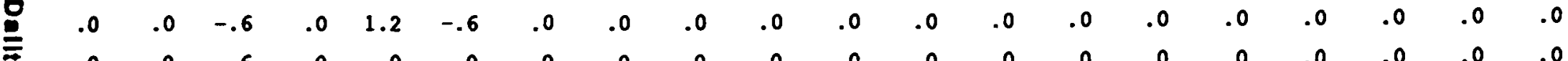

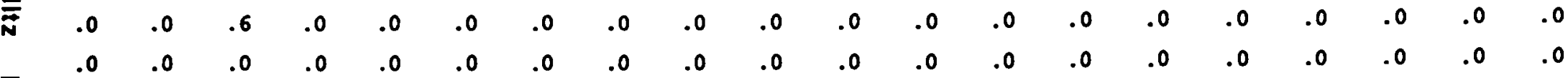

$\frac{0}{9}$ 


\section{F.2 Tabulated Data}

The vertical axis is the the kaon momentum and the horizontal axis is the $z$ of the decay vertex. The momentum bins are $10 \mathrm{GeV}$ wide, starting at $130 \mathrm{Gev}$ in the upper left hand corner and the vertex bins are $0.5 \mathrm{~m}$ wide, starting at $2 \mathrm{~m}$ in the upper left hand corner. The data listed in these tables have been corrected for collimator produced kaon backgrounds (see Appendix C).

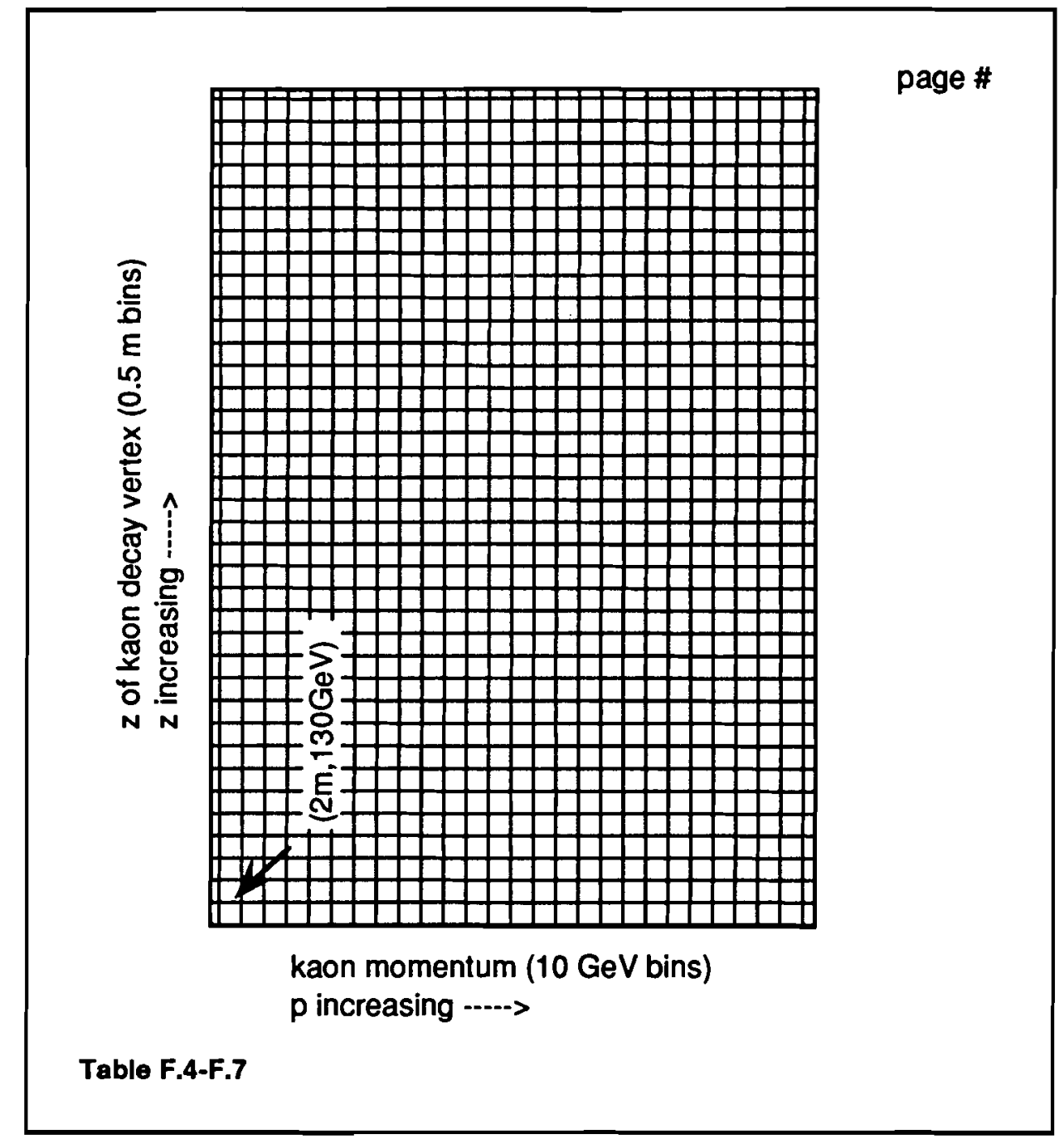




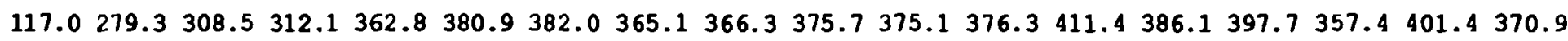

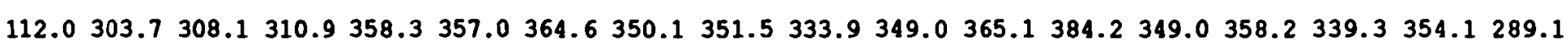

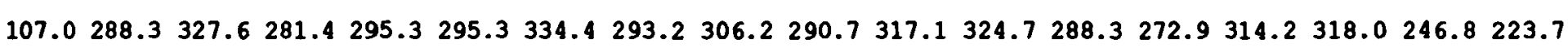
$97.0242 .8250 .2 \quad 260.3270 .2 \quad 270.8275 .1293 .0 \quad 295.9292 .4 \quad 289.3 \quad 248.7249 .6279 .4221 .3 \quad 236.3 \quad 239.4 \quad 196.3$ $93.0186 .5227 .4207 .1240 .6237 .6236 .0215 .3262 .9247 .3260 .4218 .1237 .6215 .7200 .1199 .1 \quad 188.0171 .9$ $67.0211 .9201 .0198 .1206 .8 \quad 198.0223 .9191 .5 \quad 158.3202 .8 \quad 209.5 \quad 177.0195 .1 \quad 167.5173 .3 \quad 155.9 \quad 126.0138 .8$

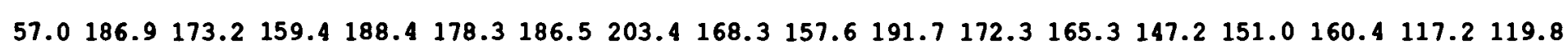

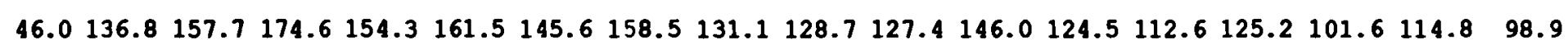
$\begin{array}{lllllllllllllllllllllll}37.0 & 102.5 & 115.2 & 153.1 & 131.9 & 114.8 & 119.0 & 131.8 & 122.0 & 125.9 & 109.1 & 127.2 & 114.0 & 98.3 & 97.7 & 103.2 & 87.7 & 82.5\end{array}$

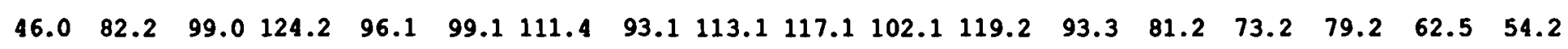

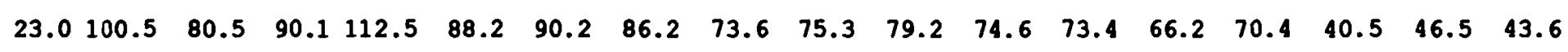
$\begin{array}{llllllllllllllllll}21.0 & 66.2 & 86.4 & 79.2 & 73.2 & 77.6 & 59.2 & 78.4 & 76.6 & 77.4 & 73.3 & 57.5 & 77.6 & 51.6 & 63.3 & 52.7 & 42.6 & 37.7\end{array}$ $\begin{array}{llllllllllllllllll}26.0 & 56.5 & 52.5 & 50.4 & 76.1 & 60.2 & 47.2 & 51.4 & 55.2 & 45.6 & 47.6 & 60.5 & 34.8 & 45.6 & 39.5 & 27.5 & 26.7 & 28.7\end{array}$ $\begin{array}{llllllllllllllllll}24.0 & 54.6 & 55.8 & 53.2 & 53.5 & 51.5 & 54.9 & 59.6 & 63.3 & 45.3 & 36.7 & 38.6 & 34.6 & 30.6 & 40.6 & 23.5 & 21.7 & 26.7\end{array}$ $\begin{array}{llllllllllllllllll}20.0 & 26.8 & 38.6 & 42.5 & 45.7 & 41.6 & 37.7 & 32.7 & 47.6 & 29.7 & 37.7 & 28.6 & 25.6 & 25.8 & 22.9 & 18.6 & 16.8 & 16.9\end{array}$ $\begin{array}{llllllllllllllllll}11.0 & 25.7 & 29.9 & 42.7 & 36.4 & 23.8 & 37.8 & 32.8 & 23.8 & 30.7 & 27.7 & 23.0 & 20.8 & 22.9 & 15.7 & 12.9 & 8.8 & 5.0\end{array}$ $\begin{array}{llllllllllllllllll}10.0 & 19.9 & 21.8 & 30.6 & 20.9 & 29.6 & 26.7 & 17.6 & 20.8 & 23.8 & 19.8 & 14.8 & 18.9 & 11.7 & 8.8 & 16.8 & 13.9 & 9.8\end{array}$ $\begin{array}{llllllllllllllllll}7.0 & 20.7 & 22.7 & 19.6 & 14.0 & 13.7 & 18.7 & 14.0 & 27.9 & 15.0 & 17.9 & 17.9 & 10.8 & 11.9 & 10.9 & 7.8 & 6.8 & 9.9\end{array}$ $\begin{array}{llllllllllllllllll}4.0 & 14.9 & 19.9 & 15.7 & 21.8 & 12.8 & 21.8 & 11.7 & 21.0 & 8.9 & 12.0 & 13.0 & 8.9 & 8.0 & 9.9 & 9.0 & 12.0 & 4.0\end{array}$ $\begin{array}{lllllllllllllllllll}7.0 & 8.9 & 7.9 & 4.9 & 13.0 & 13.0 & 12.9 & 12.0 & 13.0 & 11.9 & 7.7 & 11.9 & 7.9 & .9 & 4.9 & 3.0 & 4.9 & 3.0\end{array}$ $\begin{array}{llllllllllllllllllll}4.0 & 8.0 & 10.9 & 7.9 & 8.0 & 6.0 & 2.9 & 6.9 & 10.0 & 3.9 & 6.0 & 10.0 & 8.0 & 6.9 & 7.0 & 3.0 & .0 & 1.0\end{array}$ $\begin{array}{llllllllllllllllll}2.0 & 9.0 & 9.0 & 7.0 & 5.9 & 9.8 & 6.0 & 6.9 & 5.0 & 4.0 & 1.9 & 4.0 & 8.0 & 3.0 & 3.0 & 2.9 & 1.0 & 2.0\end{array}$ 


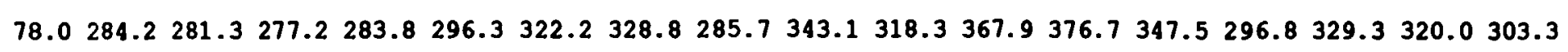
$114.0258 .7278 .9275 .4289 .3 \quad 318.5 \quad 279.5 \quad 305.5 \quad 360.4345 .7300 .2318 .5336 .7308 .2 \quad 301.1275 .8 \quad 305.1294 .1$ 100.0238 .9270 .2271 .9289 .8280 .7299 .4293 .7281 .1263 .4302 .7281 .6305 .5257 .6272 .8240 .8262 .7254 .4

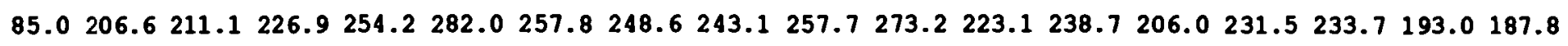

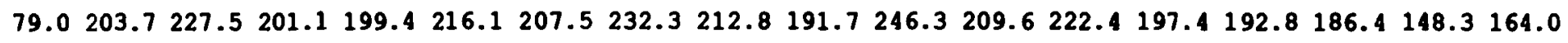

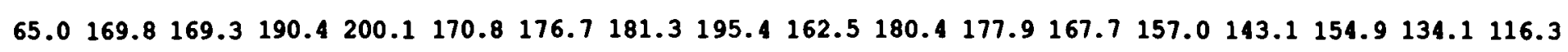

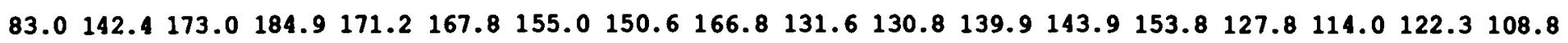

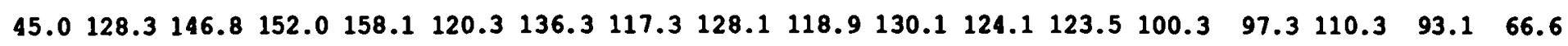
$\begin{array}{llllllllllllllllll}41.0 & 117.5 & 100.9 & 116.9 & 132.5 & 113.2 & 121.1 & 109.5 & 115.1 & 139.6 & 109.3 & 120.1 & 107.3 & 84.6 & 74.7 & 83.7 & 88.7 & 78.5\end{array}$

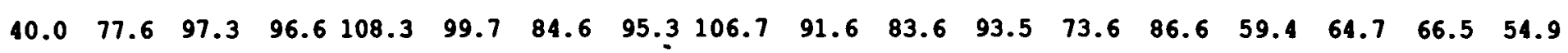
$\begin{array}{lllllllllllllllllll}35.0 & 80.6 & 68.7 & 74.7 & 80.8 & 91.6 & 75.6 & 76.8 & 79.6 & 83.4 & 75.7 & 61.5 & 60.6 & 67.5 & 57.8 & 59.8 & 53.8 & 47.8\end{array}$ $\begin{array}{lllllllllllllllllll}34.0 & 66.9 & 70.6 & 81.7 & 74.7 & 63.5 & 60.8 & 63.9 & 72.7 & 76.8 & 59.8 & 54.9 & 45.7 & 50.8 & 52.8 & 36.8 & 45.9 & 31.9\end{array}$ $\begin{array}{llllllllllllllllllll}17.0 & 65.8 & 68.8 & 57.9 & 55.7 & 62.6 & 44.7 & 53.8 & 50.7 & 55.8 & 39.0 & 56.8 & 50.8 & 34.9 & 34.9 & 36.9 & 30.8 & 25.7\end{array}$ $\begin{array}{lllllllllllllllllllll}19.0 & 42.0 & 38.8 & 50.8 & 38.8 & 41.9 & 48.8 & 34.6 & 39.8 & 37.7 & 48.7 & 41.8 & 25.8 & 31.9 & 28.9 & 26.0 & 20.0 & 20.8\end{array}$

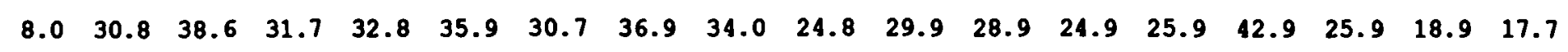
$\begin{array}{llllllllllllllllll}14.0 & 35.8 & 37.8 & 29.9 & 27.8 & 39.8 & 28.8 & 22.8 & 18.8 & 27.8 & 15.0 & 19.0 & 18.0 & 16.9 & 14.9 & 19.0 & 13.9 & 16.0\end{array}$ $\begin{array}{lllllllllllllllllll}12.0 & 11.0 & 26.9 & 26.9 & 21.9 & 20.0 & 17.0 & 21.9 & 14.9 & 22.0 & 18.9 & 12.9 & 15.0 & 14.0 & 6.0 & 13.9 & 11.0 & 9.0\end{array}$ $\begin{array}{llllllllllllllllllll}10.0 & 14.0 & 17.9 & 14.9 & 18.9 & 20.0 & 15.0 & 13.9 & 18.0 & 15.9 & 15.9 & 11.0 & 11.0 & 20.0 & 13.0 & 10.0 & 7.0 & 6.0\end{array}$ $\begin{array}{llllllllllllllllll}5.0 & 22.9 & 11.0 & 16.0 & 8.9 & 16.0 & 9.0 & 20.0 & 12.0 & 8.9 & 2.0 & 10.0 & 9.0 & 7.0 & 14.0 & 11.0 & 9.9 & 2.0\end{array}$ $\begin{array}{llllllllllllllllll}.0 & 5.0 & 10.9 & 7.0 & 9.0 & 9.0 & 8.0 & 4.0 & 10.9 & 11.0 & 11.0 & 13.0 & 4.0 & 7.0 & 2.0 & 4.0 & 2.0 & .0\end{array}$ $\begin{array}{llllllllllllllllll}3.0 & 10.0 & 7.0 & 8.0 & 4.8 & 4.0 & 9.0 & 14.0 & 13.0 & 6.0 & 5.0 & 4.0 & 2.0 & 3.0 & 3.0 & 4.0 & 2.0 & 2.0\end{array}$

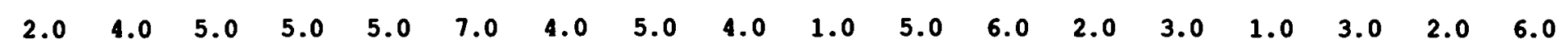




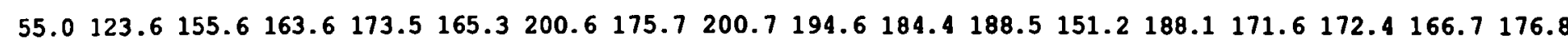

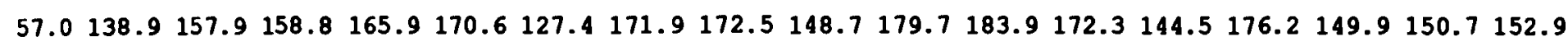
$54.0141 .4136 .7149 .6168 .7 \quad 140.9170 .9149 .6146 .8 \quad 155.7 \quad 144.9160 .7 \quad 165.5158 .0142 .4139 .5132 .6122 .7$ $\begin{array}{lllllllllllllllllll}58.0 & 126.0 & 133.7 & 125.8 & 125.9 & 143.7 & 129.4 & 115.8 & 130.9 & 150.9 & 126.7 & 136.7 & 132.9 & 126.7 & 116.7 & 106.9 & 105.8 & 96.7\end{array}$

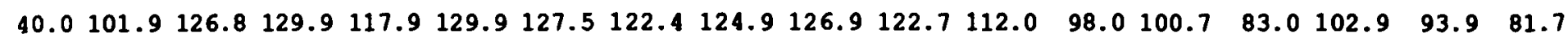

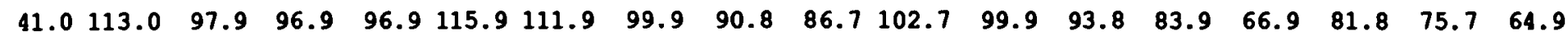

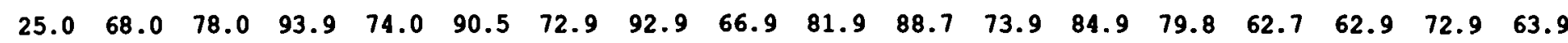
$\begin{array}{llllllllllllllllll}18.0 & 81.9 & 66.0 & 77.0 & 67.0 & 80.0 & 76.9 & 78.9 & 69.0 & 64.9 & 67.0 & 60.0 & 65.0 & 47.9 & 50.9 & 52.8 & 55.8 & 44.0\end{array}$

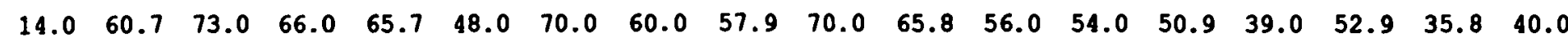
$\begin{array}{lllllllllllllllllll}19.0 & 59.9 & 62.8 & 59.0 & 48.0 & 38.0 & 47.7 & 53.0 & 34.0 & 46.7 & 51.0 & 44.0 & 37.8 & 30.0 & 36.0 & 36.0 & 33.0 & 28.0\end{array}$

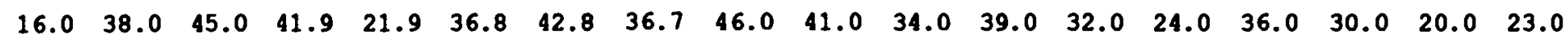
$\begin{array}{llllllllllllllllll}14.0 & 39.9 & 24.0 & 42.0 & 33.8 & 38.8 & 34.9 & 30.8 & 20.0 & 31.0 & 27.0 & 29.8 & 22.0 & 21.0 & 16.0 & 22.0 & 18.0 & 16.0\end{array}$ $\begin{array}{llllllllllllllllllll}7.0 & 26.0 & 24.0 & 38.0 & 32.9 & 33.9 & 26.9 & 16.0 & 30.0 & 31.0 & 24.0 & 20.0 & 15.9 & 22.0 & 16.0 & 17.8 & 8.0 & 16.0\end{array}$ $\begin{array}{llllllllllllllllll}10.0 & 22.0 & 22.0 & 32.0 & 26.0 & 27.0 & 26.9 & 26.0 & 14.9 & 22.0 & 28.0 & 16.0 & 22.0 & 13.0 & 15.0 & 16.0 & 13.0 & 10.0\end{array}$

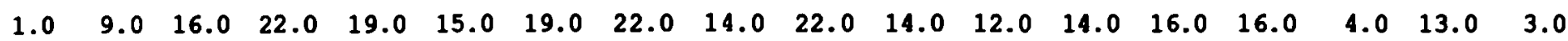
$\begin{array}{llllllllllllllllll}5.0 & 17.0 & 11.0 & 12.0 & 15.0 & 22.8 & 16.0 & 21.0 & 11.0 & 13.0 & 13.0 & 5.0 & 13.0 & 13.0 & 4.0 & 10.0 & 6.0 & 11.0\end{array}$

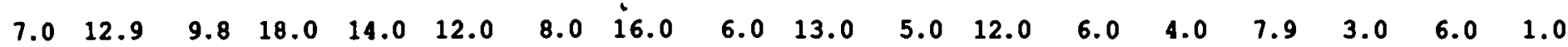
$\begin{array}{llllllllllllllllll}7.0 & 3.0 & 11.0 & 18.0 & 10.0 & 10.0 & 9.0 & 8.0 & 8.0 & 7.0 & 11.9 & 8.0 & 9.0 & 5.0 & 2.0 & 5.0 & 1.0 & 3.0\end{array}$ $\begin{array}{llllllllllllllllll}2.0 & 15.0 & 5.0 & 6.0 & 8.0 & 7.0 & 5.0 & 6.0 & 4.0 & 7.0 & 3.0 & 4.0 & 4.0 & 7.0 & 1.0 & 3.0 & 2.0 & 1.0\end{array}$ $\begin{array}{llllllllllllllllll}.0 & 4.0 & 5.0 & 5.0 & 5.0 & 8.0 & 4.0 & 7.0 & 9.0 & 3.0 & 5.0 & 2.0 & 4.0 & 3.0 & 4.0 & 2.0 & 3.0 & .0\end{array}$

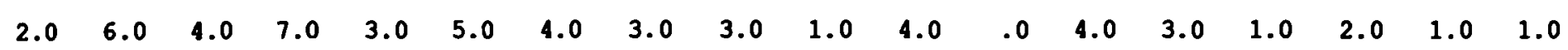

$\begin{array}{llllllllllllllllll}.0 & 1.0 & 4.0 & 3.0 & 7.0 & 3.0 & 4.0 & 2.0 & 5.0 & 1.0 & 3.0 & .0 & 5.0 & 3.0 & 4.0 & .0 & 2.0 & 1.0\end{array}$

1


$53.0128 .7150 .8 \quad 149.8160 .8 \quad 147.8 \quad 157.8 \quad 146.8 \quad 154.9 \quad 165.7 \quad 162.6 \quad 175.8 \quad 166.8 \quad 165.8 \quad 172.8 \quad 174.7 \quad 175.7 \quad 174.7$ $\begin{array}{llllllllllllllllll}49.0 & 121.9 & 125.8 & 166.8 & 157.8 & 133.8 & 152.7 & 178.9 & 152.7 & 151.7 & 177.7 & 140.8 & 162.8 & 143.6 & 151.7 & 153.7 & 134.8 & 131.7\end{array}$

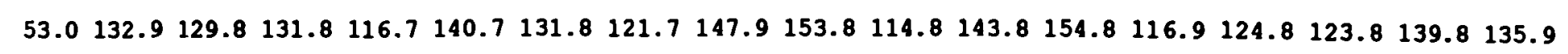

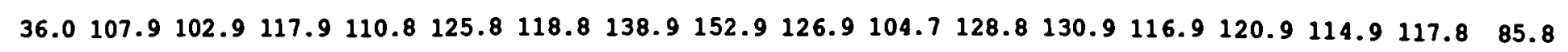
$\begin{array}{llllllllllllllllll}28.0 & 105.9 & 78.0 & 93.9 & 106.8 & 103.9 & 110.9 & 106.8 & 111.8 & 100.9 & 121.9 & 91.9 & 95.9 & 74.8 & 90.8 & 88.8 & 84.9 & 78.9\end{array}$

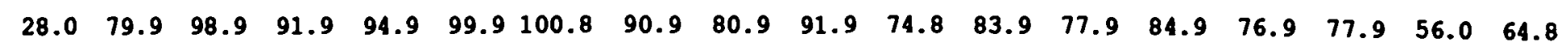
$\begin{array}{llllllllllllllllll}22.0 & 92.9 & 76.9 & 79.9 & 64.0 & 78.0 & 81.9 & 86.9 & 72.9 & 87.9 & 85.9 & 79.9 & 60.9 & 52.0 & 72.9 & 55.0 & 59.0 & 46.9\end{array}$ $\begin{array}{lllllllllllllllllll}19.0 & 64.0 & 70.9 & 71.9 & 52.9 & 68.0 & 81.9 & 57.9 & 57.9 & 65.9 & 63.9 & 58.8 & 67.9 & 41.9 & 63.9 & 55.0 & 47.9 & 36.0\end{array}$ $\begin{array}{lllllllllllllllllll}25.0 & 47.0 & 63.0 & 56.0 & 54.0 & 53.0 & 47.0 & 44.0 & 66.0 & 57.0 & 46.0 & 47.9 & 51.0 & 58.9 & 46.0 & 52.0 & 48.0 & 32.0\end{array}$ $\begin{array}{lllllllllllllllllll}22.0 & 44.9 & 30.9 & 51.0 & 45.9 & 43.0 & 43.9 & 44.0 & 45.9 & 52.0 & 43.0 & 39.0 & 39.0 & 38.0 & 38.9 & 38.0 & 32.0 & 23.0\end{array}$ $\begin{array}{lllllllllllllllllll}11.0 & 44.9 & 46.0 & 54.0 & 35.9 & 25.0 & 54.0 & 37.0 & 41.0 & 36.0 & 23.9 & 36.0 & 24.9 & 43.0 & 37.0 & 28.0 & 20.0 & 14.0\end{array}$ $\begin{array}{lllllllllllllllllll}17.0 & 39.0 & 31.0 & 36.0 & 30.0 & 37.0 & 29.0 & 33.0 & 27.0 & 26.0 & 38.0 & 16.0 & 30.0 & 23.0 & 33.0 & 17.0 & 11.0 & 24.0\end{array}$ $\begin{array}{lllllllllllllllllll}10.0 & 28.0 & 31.0 & 23.0 & 33.9 & 28.0 & 24.0 & 33.0 & 24.0 & 25.0 & 33.0 & 21.0 & 16.0 & 17.9 & 22.0 & 13.0 & 17.0 & 16.0\end{array}$ $\begin{array}{lllllllllllllllllll}13.0 & 22.0 & 24.0 & 27.0 & 28.0 & 22.0 & 28.0 & 30.0 & 16.0 & 15.0 & 18.0 & 18.0 & 10.0 & 17.9 & 16.0 & 10.0 & 13.0 & 14.0\end{array}$ $\begin{array}{llllllllllllllllll}6.0 & 19.0 & 19.0 & 20.0 & 26.0 & 19.0 & 16.0 & 11.0 & 13.0 & 15.0 & 8.0 & 14.0 & 14.0 & 9.0 & 13.0 & 10.0 & 17.0 & 9.0\end{array}$ $\begin{array}{llllllllllllllllllll}9.0 & 12.0 & 18.0 & 11.0 & 20.0 & 11.0 & 10.0 & 14.0 & 12.0 & 14.0 & 12.0 & 11.0 & 6.0 & 6.0 & 10.0 & 3.0 & 6.0 & 9.0\end{array}$ $\begin{array}{llllllllllllllllllll}9.0 & 10.0 & 10.0 & 13.0 & 8.0 & 7.0 & 7.9 & 10.0 & 10.0 & 8.0 & 4.0 & 7.0 & 12.0 & 2.0 & 5.0 & 7.0 & 7.0 & 6.0\end{array}$ $\begin{array}{lllllllllllllllllll}2.0 & 11.0 & 11.0 & 5.0 & 12.0 & 11.0 & 15.0 & 13.0 & 6.0 & 9.0 & 6.0 & 4.0 & 3.0 & 10.0 & 4.0 & 3.0 & 2.0 & 3.0\end{array}$ $\begin{array}{lllllllllllllllllll}3.0 & 1.0 & 10.0 & 3.0 & 10.0 & 6.0 & 6.0 & 3.0 & 5.0 & 2.0 & 5.0 & 7.0 & 4.0 & 2.0 & 3.0 & 3.0 & 6.0 & 5.0\end{array}$ $\begin{array}{lllllllllllllllllll}2.0 & 3.0 & 3.0 & 8.0 & 7.0 & 6.0 & 2.0 & 5.0 & 7.0 & 9.0 & 7.0 & 5.0 & 2.0 & 6.0 & 2.0 & 1.0 & 3.0 & 2.0\end{array}$ $\begin{array}{rrrrrrrrrrrrrrrrrrr}.0 & 4.0 & 5.0 & 4.0 & 5.0 & 5.0 & 2.0 & 3.0 & 5.0 & 6.0 & .0 & 3.0 & 2.0 & 2.0 & 1.0 & 3.0 & .0 & 1.0\end{array}$ $\begin{array}{lllllllllllllllllll}2.0 & .0 & 3.0 & .0 & 4.0 & 2.0 & 1.0 & 1.0 & 6.0 & 1.0 & 2.0 & 4.0 & 2.0 & 1.0 & 1.0 & 2.0 & 2.0 & 1.0\end{array}$ 


\section{F.3 Tabulated Acceptance}

The vertical axis is the the kaon momentum and the horizontal axis is the $z$ of the decay vertex. The momentum bins are $10 \mathrm{GeV}$ wide, starting at $130 \mathrm{Gev}$ in the upper left hand corner and the vertex bins are $0.5 \mathrm{~m}$ wide, starting at $2 \mathrm{~m}$ in the upper left hand corner.

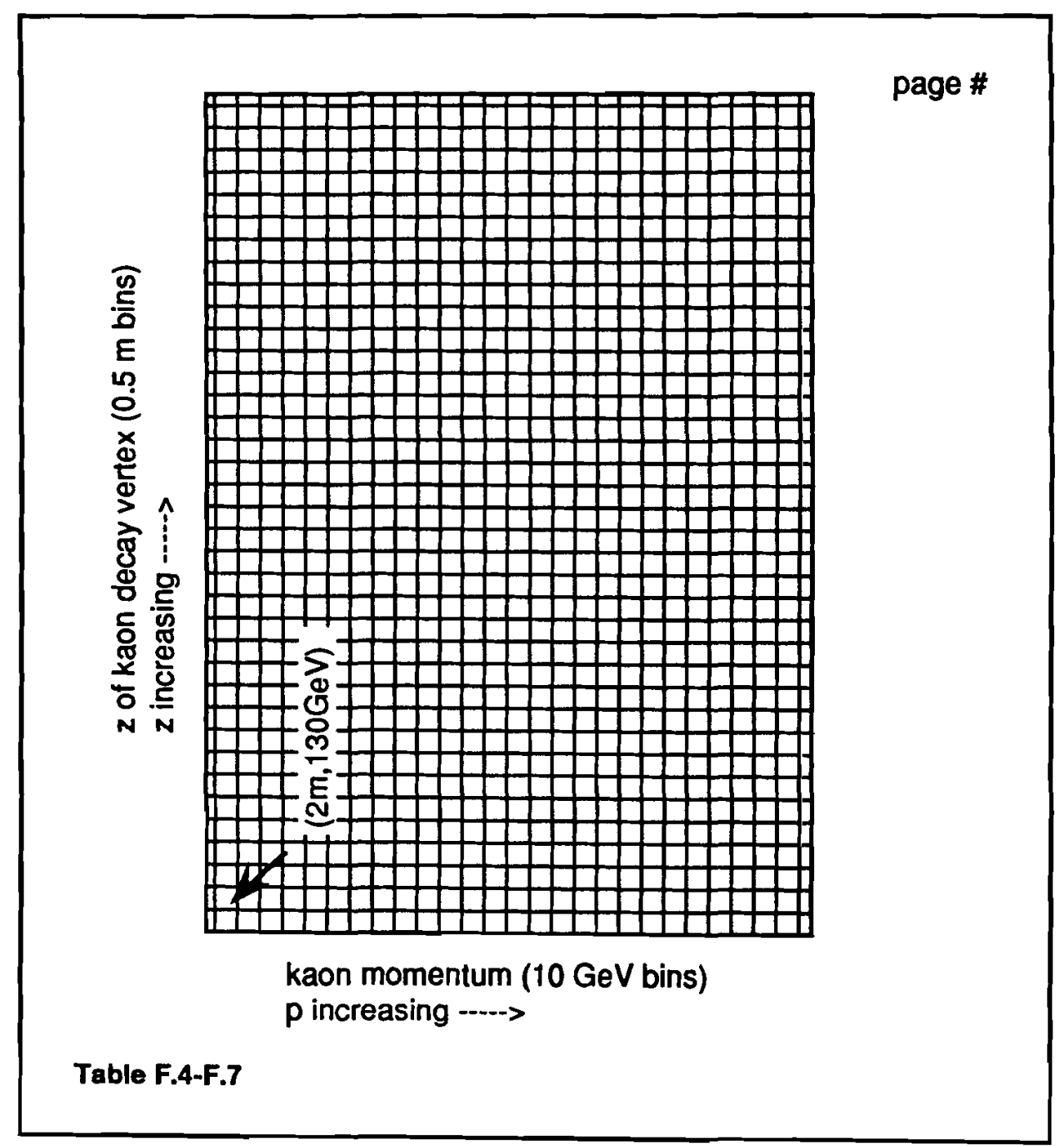




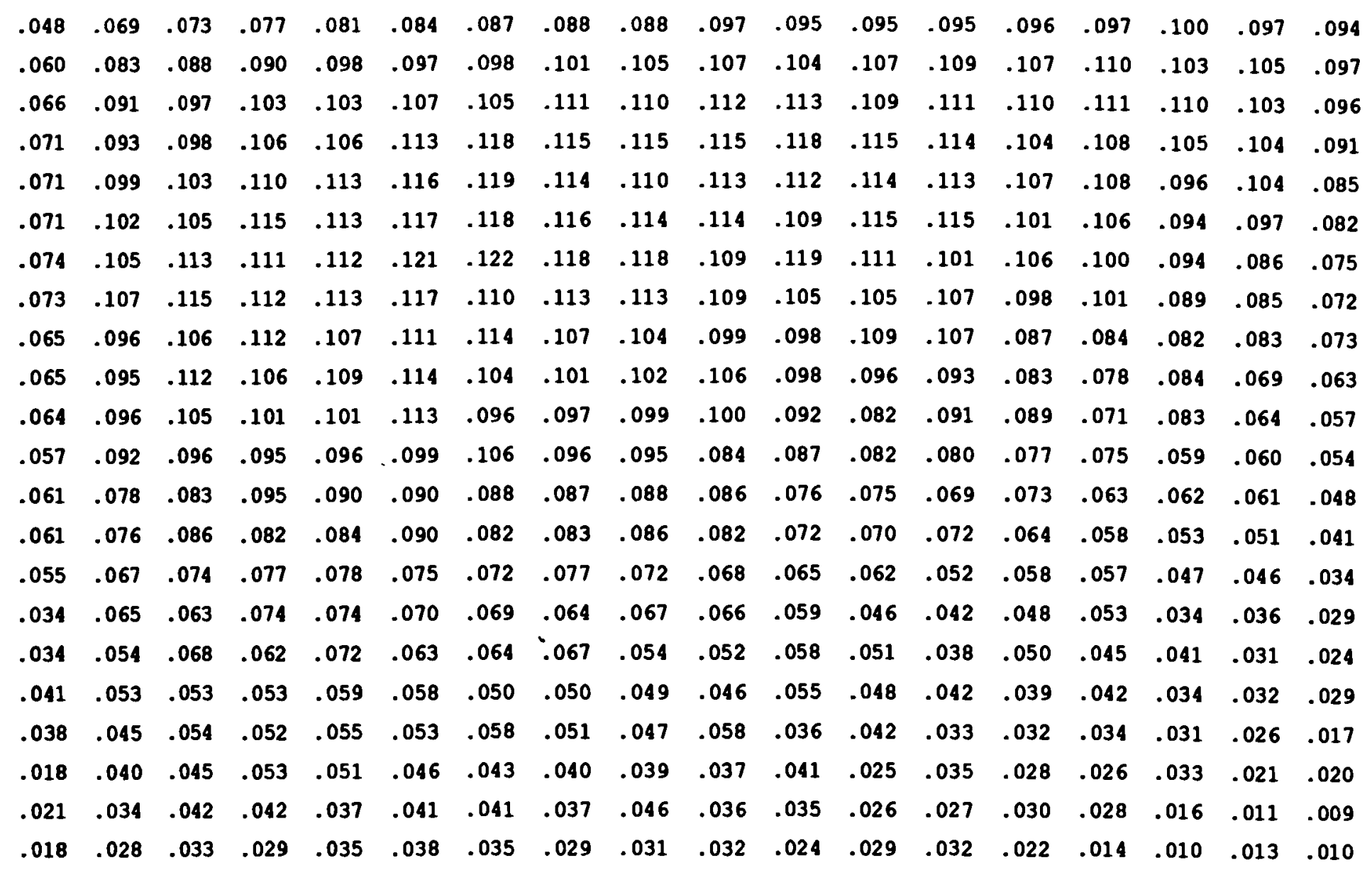




\begin{tabular}{|c|c|c|c|c|c|c|c|c|c|c|c|c|c|c|c|c|c|}
\hline 037 & 056 & 059 & .062 & .065 & 067 & 072 & 071 & 073 & 075 & .076 & .076 & 080 & .079 & .077 & .081 & .078 & .07 \\
\hline 047 & .066 & .073 & .072 & .076 & .079 & .080 & .079 & .082 & .082 & .085 & .086 & .086 & .085 & .086 & .082 & .081 & .07 \\
\hline .053 & .075 & .082 & .080 & .082 & .089 & .088 & .089 & .089 & .088 & .088 & .090 & .089 & .088 & .089 & .087 & .085 & .07 \\
\hline 051 & 077 & . 0866 & . 087 & .091 & 090 & 093 & .092 & .094 & 090 & .092 & .090 & .092 & .091 & .089 & .087 & .081 & 0 \\
\hline .060 & .083 & .084 & .090 & .092 & 093 & 097 & .089 & 094 & 089 & .091 & .092 & 087 & .088 & .086 & .083 & .082 & .07 \\
\hline 60 & .085 & .088 & .091 & .088 & 094 & 092 & .091 & .090 & 089 & .089 & .086 & 087 & .087 & .083 & .080 & .077 & .0 \\
\hline . 055 & .083 & 087 & .089 & .099 & 091 & 089 & .094 & .095 & 090 & .093 & .085 & .086 & .082 & .080 & .077 & .069 & .06 \\
\hline .058 & 081 & 090 & .092. & .091 & .088 & 093 & .094 & .088 & .087 & .087 & .086 & .082 & .082 & .075 & .073 & .066 & .06 \\
\hline .066 & 080 & .090 & .089. & .089 & .094 & 092 & .089 & .092 & 086 & .083 & .086 & . 078. & .073 & .073 & .069 & .065 & .05 \\
\hline 062 & .077 & .090 & .090. & .086 & 088 & 086 & .090 & .082 & 090 & .081 & .084 & . 072 & .072 & .070 & .063 & .059 & .04 \\
\hline .062 & .081 & .083 & .091 & .087 & .086 & .085 & .083 & .078 & . 077 & .076 & .075 & .071 & .069 & .069 & .061 & .053 & .0 \\
\hline .052 & .072 & .081 & .077 & .079 & .078 & .081 & . 079 & .074 & .077 & .069 & .068 & .069 & .061 & .058 & .057 & .053 & .0 \\
\hline .048 & .066 & .081 & .075 & .077 & .077 & .072 & .073 & .078 & .070 & .067 & .067 & .061 & .058 & .056 & .051 & .043 & 0 \\
\hline . 044 & .063 & .071 & .072 & .070 & .074 & .067 & .071 & .066 & .065 & .058 & .058 & .058 & .053 & .047 & .045 & .041 & \\
\hline .043 & .062 & .066 & .065 & .062 & .066 & . 064 & .061 & .062 & .060 & .060 & .050 & .053 & .050 & .043 & .039 & .043 & 0 \\
\hline .041 & .051 & .060 & .055 & .063 & .061 & .060 & :056 & .053 & .056 & .054 & .050 & .044 & .046 & .041 & .036 & .028 & .0 \\
\hline .040 & .051 & .048 & .055 & .059. & .052 & .055 & .051 & .050 & .046 & .048 & .040 & .043 & .037 & .036 & .034 & .027 & .0 \\
\hline .023 & .047 & .050 & .052 & .046 & .045 & .047 & .042 & .045 & .047 & .039. & .038 & .038 & .034 & .028 & .034 & .026 & .0 \\
\hline .024 & .041 & .042 & .050 & .045 & .041 & . 051 & .051 & .035 & .043 & .032 & .035 & .031 & .029 & .029 & .025 & .020 & \\
\hline .028 & .038 & .040 & .038 & .046 & .046 & .046 & .033 & .036 & .035 & .034 & .033 & .027 & .025 & .027 & .021 & .017 & \\
\hline . 023 & .028 & .038 & .036 & .038 & .038 & .030 & .034 & .030 & .034 & .030 & .020 & .025 & .024 & .018 & .021 & .015 & \\
\hline . 022 & 020 & 029 & 028 & 034 & .032 & 030 & .024 & .023 & .025 & .021 & .023 & .021 & .013 & . 017. & .012 & .018 & \\
\hline
\end{tabular}

$\begin{array}{lllllllllllllllllll}1 & 1 & 1 & 1 & 1 & 1 & 1 & 1 & 1 & 1 & 1 & 1 & 1 & 1 & 1 & 1 & 1 & 1 & 1\end{array}$




\begin{tabular}{|c|c|c|c|c|c|c|c|c|c|c|c|c|c|c|c|c|c|}
\hline .038 & .054 & .058 & .064 & .062 & .067 & .072 & .071 & .074 & .077 & .079 & .082 & .080 & .081 & .076 & .075 & .080 & .074 \\
\hline .049 & .066 & . 073 & .075 & .080 & .079 & .083 & .083 & .079 & .087 & .082 & .086 & .084 & .087 & .084 & .081 & .078 & .075 \\
\hline . 050 & .072 & .076 & .082 & .090 & .085 & .086 & .087 & .089 & .089 & .086 & .087 & .090 & .096 & .091 & .087 & .083 & .076 \\
\hline .053 & .077 & .082 & .091 & .089 & .089 & . 089 & .097 & .090 & .095 & .087 & .091 & .084 & .089 & .093 & .081 & .084 & .073 \\
\hline .057 & .079 & .085 & .090 & .095 & .091 & .090 & .088 & .095 & .097 & .096 & .093 & .091 & .092 & .085 & .086 & .078 & .071 \\
\hline .062 & .084 & .093 & .092 & .090 & .096 & .088 & .093 & .086 & .095 & .093 & .090 & .089 & .087 & .075 & .081 & .073 & .065 \\
\hline .058 & .083 & .094 & .088 & .099 & .092 & .099 & .091 & .095 & .094 & .082 & .094 & .087 & .082 & .082 & .076 & .076 & .063 \\
\hline .071 & .082 & .086 & .095 & .099 & .093 & .090 & .086 & .086 & .089 & .091 & .084 & .083 & .081 & .082 & .074 & .063 & .062 \\
\hline .058 & .080 & .087 & .096 & .095 & .098 & .090 & .085 & .091 & .089 & .086 & .083 & .074 & .075 & .080 & .062 & .062 & .060 \\
\hline .062 & .075 & .090 & .096 & .095 & .092 & .092 & .085 & .089 & .072 & .076 & .087 & .084 & .064 & .069 & .070 & .062 & .048 \\
\hline .054 & .078 & .083 & .094 & .085 & .082 & .088 & .076 & .084 & .078 & .078 & .071 & .071 & .068 & .061 & .054 & .046 & .053 \\
\hline .054 & .070 & .081 & .075 & .081 & .077 & .085 & .070 & .088 & .076 & .062 & .076 & .068 & .060 & .057 & .056 & .050 & .044 \\
\hline .044 & .079 & .074 & .076 & .071 & .079 & .071 & .074 & .067 & .068 & .061 & .068 & .052 & .063 & .053 & .053 & .046 & .038 \\
\hline .055 & .057 & .068 & .061 & .063 & .064 & .065 & .066 & .081 & .067 & .066 & .056 & .052 & .051 & .052 & .036 & .035 & .036 \\
\hline .046 & .060 & .062 & .072 & .064 & .065 & .058 & .063 & .053 & .057 & .046 & .048 & .050 & .049 & .051 & .038 & .030 & .030 \\
\hline .035 & .051 & .054 & .066 & .060 & .052 & .059. & .061 & .051 & .059 & .050 & .053 & .047 & .038 & .041 & .041 & .027 & .020 \\
\hline .033 & .049 & .051 & .054 & .053 & .049 & .043 & .054 & .052 & .041 & .046 & .039 & .037 & .040 & .043 & .039 & .032 & .020 \\
\hline .026 & .051 & .050 & .053 & .059 & .051 & .048 & .042 & .046 & .039 & .038 & .038 & .036 & .032 & .038 & .036 & .027 & .020 \\
\hline .031 & .040 & .050 & .046 & .052 & .042 & .041 & .038 & .035 & .040 & .033 & .034 & .032 & .034 & .017 & .026 & .023 & .018 \\
\hline .015 & .035 & .042 & .036 & .061 & .038 & .030 & .029 & .037 & .040 & .039 & .034 & .022 & .021 & .021 & .023 & .025 & .015 \\
\hline .020 & .042 & .040 & .045 & .032 & .049 & .037 & .026 & .026 & .037 & .023 & .031 & .024 & .010 & .020 & .015 & .015 & .007 \\
\hline .018 & .032 & .030 & .037 & .032 & .033 & .025 & .031 & .020 & .028 & .028 & .030 & .020 & .017 & .011 & .010 & . 007 & .010 \\
\hline
\end{tabular}




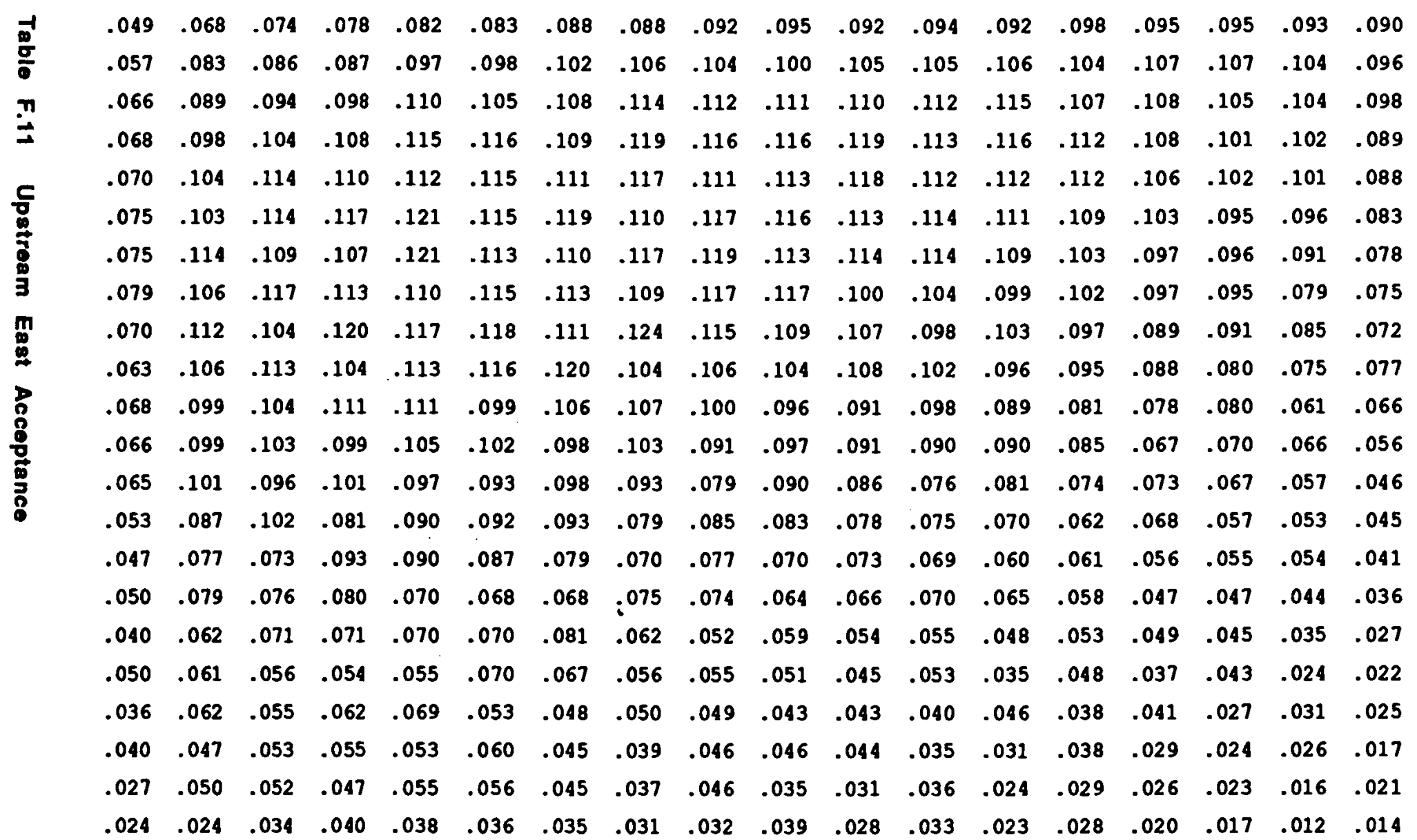

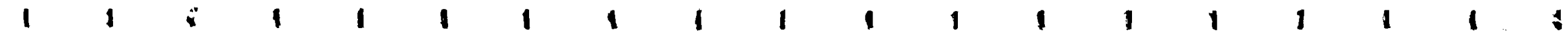




\section{References}

(1) G. Luders, Ann. Phys. 2 (1957) 1.

W. Pauli, In: Niels Bohr and the Development of Physics, ed. W. Pauli, Oxford: Pergamon (1955) 2nd ed. p. 30.

(2) T. D. Lee and C. N. Yang, Phys. Rev. 104 (1956) 254.

(3) C. S. Wu et al., Phys. Rev. 105 (1957) 1413.

(4) R. L. Garwin, L. M. Lederman and M. Weinrich, Phys. Rev. 105 (1957) 1415.

J. I. Friedman and V. L. Telegdi, Phys. Rev. 105 (1957) 1681.

(5) L. D. Landau, Nucl. Phys. 3 (1957) 127.

(6). M. Gell-Mann and A. Pais, Phys. Rev. 97 (1955) 1387.

(7) M. Bardon et al., Ann. Phys. NY 5 (1958) 156.

D. Neagu et al., Phys. Rev. Lett. 6 (1961) 552.

(8) J. H. Christenson, J. W. Cronin, V. L. Fitch and R. Turlay, Phys. Rev. Lett. 13 (1964) 138.

(9) J. M. Gaillard et al., Phys. Rev. Lett. 18 (1967) 20.

J. W. Cronin et al., Phys. Rev. Lett. 18 (1967) 25.

(10) S. Bennet et al., Phys. Rev. Lett. 19 (1967) 993.

(11) R. Turlay, Saclay preprint DPhPE (1986) 86-20.

(12) Particle Data Group, Physics Letters B 204 (1988) p186.

(13) J. F. Donoghue, University of Massachusetts preprint (1988) UMHEP. 311.

(14) M. Kobayashi and K. Maskawa, Prog. Theor. Phys. 49 (1973) 652.

(15) L. Wolfenstein, Phys. Rev. Lett. 13 (1964) 18562.

(16) L. Maiani, in: Proc. 1977 Intern. Symp. on Lepton and Photon Interactions at High Energies (DESY, Hamburg, 1977), p. 867.

L. Wolfenstein, Phys. Rev. Lett. 51(1983) 1945.

(17) J. F. Donoghue et al., Physics Reports 131 Nos. 586 (1986) 319-428.

(18) L. Wolfenstein, Ann. Rev. Nucl. Part. Sci. 36 (1986) 70p 137.

(19) L. F. Li and L. Wolfenstein, Phys. Rev. D21 (1980)1 178. 
(20) J. F. Donoghue, B. R. Holstein and G. Valencia, Phys. Rev. D36 (1987) 3 798.

(21) S. Fajfer and J.-M. G,erard, Z. Phys. C- Particles and Fields 42 (1989) 425.

(22) J. R. Patterson et al., Phys. Rev. Lett. 64 (1990) 1491.

(23) S. Weinberg, Phys. Rev. Lett. 37 (1976) 11,675.

(24) C. Albright, J. Smith and S. H. H. Trye, Phys. Rev. D21 (1980) 711.

G. C. Branco, Phys. Rev. D22 (1980) 2901.

(25) J. F. Donoghue and B. R. Holstein, Phys. Rev. D32 (1985) 1152.

(26) A. Sanda, Moriond Workshop on Flavour Mixing and CP Violation, ed. J. T. Thanh Van. Gif-sur-Yvette:Editions Frontiers (1985) 345.

(27) H. Y. Cheng, Phys. Lett. 129B (1983) 5357.

(28) R. N. Mohapatra, NATO Summer School on Particle Physics, Sept. 1983. (1984) New York:Plenum.

G. Beal and A. Soni, In: Proc. 18 th Recontre de Moriond, ed. J. Tran Thanh Van, (1983) Gif-sur-Yvette:Editions Frontierre p. 339.

(29) R. N. Mohapatra and J. C. Pati, Phys. Rev. D11 (1975) 3,566.

(30) J. K. Black et al., Phys. Rev. Lett. 54 (1985) 1628.

(31) R. H. Bernstein et al., Phys. Rev. Lett. 54 (1985) 1631.

(32) H. Burkhardt et al., Phys. Lett. B 206 (1988) 169.

(33) A. J. Buras and J.-M. Gerard, Munich preprint MPI-PAE/Pth 84-87 (1987) and references therein.

(34) Particle Data Group, Physics Letters B 204 (1988) p196.

(35) P. Border Ph. D. thesis, "CP-Violation in the Decay Ko $\rightarrow \pi^{+} \pi^{-} \pi^{\circ \text { ", }}$ University of Michigan, (1988), p 39, unpublished

(36) G. B. Thomson FNAL Technical Memo, TM-1144 7190.621 2250.0000 (1982)

(37) K. Thorne Ph. D. thesis, "Precision Measurement of the Lifetime and Decay Asymmetry of the Cascade-Zero Baryon", Unversity of Minnesota, (1990), unpublished

(38) J.F. Bartlett et al., "RT/RSX MULTI: Packages for Data Acquisition and Analysis in High Energy Physics", Topical Conf. on Computerized Data Acquisition Systems in Particle and Nuclear Physics, Sante Fe (1979)

(39) Particle Data Group, Phys. Lett. B,204 (1988) 195.

(40) Particle Data Group, Phys. Lett. B,204 (1988) 65. 
(41) Particle Data Group, Phys. Lett. B,204 (1988) 187.

(42) F. James and M. Roos, Computer Physics Communication 10 (1975) 343.

(43) K. B. Luk et al. Phys. Rev., D34 (1986) 53.

(44) J.R. Johnson, et al., Phys. Rev. Lett. 39 (1977) 19,1173.

D. Antreasyan et al., Phys. Rev. Lett. 38 (1977) 3,112\&115.

D.S. Barton et al., Phys. Rev. D 27 (1983) 2580.

T. Eichten et al., Nuclear Physics B44 (1972) 333.

(45) R.D. Field and R.P. Feynmann, Phys. Rev. D 15 (1977) 2590.

(46) T. Eichten et al., Nuclear Physics B44 (1972) 333.

(47) T.D. Lee and C.S. Wu, Annual Review of Nuclear Science 16 (1966) 511.

(48) L.F. Li and L. Wolfenstein, Phys. Rev. D 21 (1980) 1,178. 


\section{Errata}

Some typographical errors have been corrected from the original thesis on file at the University of Minnesota. More significant corrections are shown below.

(1) page 155 , equation 9.1 , the $\left(\eta_{+-0}\right)^{2}$ term has been left out but was actually included in the Monte Carlo event generation and the final analysis

(2) page $157, \varphi_{+-0} \rightarrow-\varphi_{+-}$

(3) page 160, equation 9.16 (some signs have been corrected)

(4) page 192, DK 19.50

(5) page 204, sentence added: " The data listed in these...."

(6) page 216 , reference 44 